\title{
Palladium-Catalyzed Modular Synthesis of Substituted Piperazines and Related Nitrogen Heterocycles
}

\author{
Supporting Information \\ Thomas D. Montgomery and Viresh H. Rawal \\ Searle Chemistry Laboratory, Department of Chemistry, The University of Chicago \\ 5735 South Ellis Avenue, Chicago, IL 60637, USA \\ vrawal@uchicago.edu
}

\section{Table of Contents}

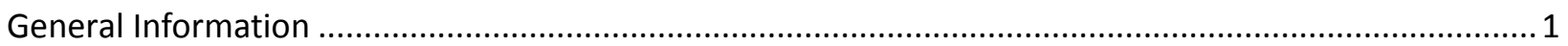

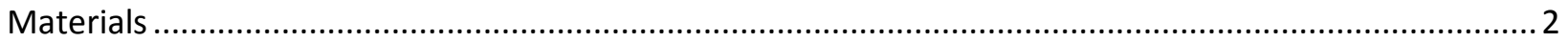

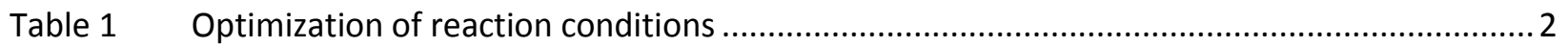

Preparation of tert-Butyl Propargyl Carbonates .................................................................................. 3

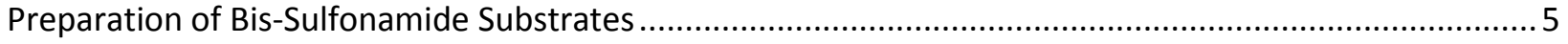

Preparation of Amino Acid Based Substrates ..................................................................................... 11

General Procedure for the Pd-catalyzed Piperizine Formation ................................................................ 19

General Procedure for the Pd-catalyzed Piperizine Formation with Substituted Propargyl Carbonates .. 32

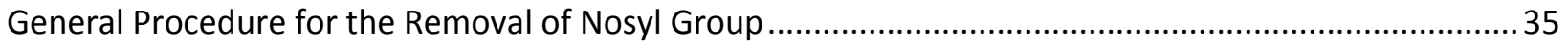

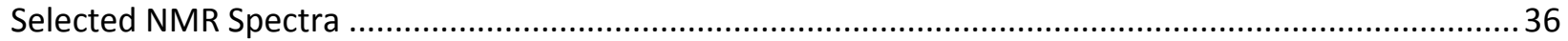

\section{General Information}

Reactions were run in oven-dried glassware under $\mathrm{N}_{2}$ atmosphere. Reactions were monitored by TLC on EMD Millipore silica gel $60 \AA$ F254 plates, visualized by UV florescence quenching $(254 \mathrm{~nm})$ or $\mathrm{KMnO}_{4}$ staining solution. Flash column chromatography (EtOAc/Hexanes or $\mathrm{MeOH} / \mathrm{CH}_{2} \mathrm{Cl}_{2}$ ) was performed on SiliCycle SiliaFlash (40-63 $\mu \mathrm{m})$. NMR spectra were measured on Bruker DRX, DMX or SMP spectrometers at $500 \mathrm{MHz}$ for ${ }^{1} \mathrm{H}$ spectra and $125 \mathrm{MHz}$ for ${ }^{13} \mathrm{C}$ spectra. ${ }^{1} \mathrm{H}$ spectra were calibrated from internal standard TMS $(\delta 0.0)$ or solvent resonance $\left(\mathrm{CHCl}_{3}: 7.26\right.$, DMSO: 2.50 , Acetone: 2.05$) .{ }^{13} \mathrm{C}$ spectra were calibrated from solvent resonance $\left(\mathrm{CHCl}_{3}\right.$ : 77.0, DMSO: 39.52, Acetone: 206.26). NMR data are reported as: chemical shift (parts per million, $p p m$ ), multiplicity $(s=$ singlet, $d=$ doublet, $t=$ triplet, $q=$ quartet, $p=$ pentet, $m=$ multiplet, $b r=$ broad signal), coupling constant $(\mathrm{Hz})$ and integration. High-resolution mass spectral analysis was measured on Agilent Technologies 6224 TOF LC/MS (positive electrospray ionization). NMR yields were determined by comparison to an internal standard as previously reported. ${ }^{1}$

${ }^{1}$ Nibbs, A. E. ; Montgomery, T. D.; Zhu, Y.; Rawal, V. H. J. Org. Chem. 2015, 80, 4928. 


\section{Materials}

Solvents: Methylene chloride $\left(\mathrm{CH}_{2} \mathrm{Cl}_{2}\right)$, tetrahydrofuran (THF) and acetonitrile (MeCN) were purified by passage over activated alumina, using and Innovative Technology, Inc. Puresolv solvent purification system. All other solvents were purchased from Fisher Scientific and used as received.

Palladium Sources: $\mathrm{Pd}_{2}(\mathrm{dba})_{3} \cdot \mathrm{CHCl}_{3}$ and $\mathrm{Pd}\left(\mathrm{PPh}_{3}\right)_{4}$ were purchased from Strem Chemicals. $\mathrm{Pd}(\mathrm{OAc})_{2}$ was purchased from Sigma-Aldrich. $\mathrm{Pd}_{2}(\mathrm{dba})_{3} \cdot \mathrm{CHCl}_{3}$ was periodically recrystallized to remove accumulated nanoparticals according to the protocol of Ananikov and coworkers. ${ }^{2}$

Phosphine ligands: DPEphos, xantphos, dppb, dppf, dppe and $\mathrm{P}(2 \text {-furyl })_{3}$ were purchased from Strem Chemical. $(R)$-BINAP and $\mathrm{P}(t-\mathrm{Bu})_{3}$ were purchased from Sigma-Aldrich.

Unless otherwise noted, materials were purchased from commercial suppliers and used as received.

Table 1 Optimization of reaction conditions

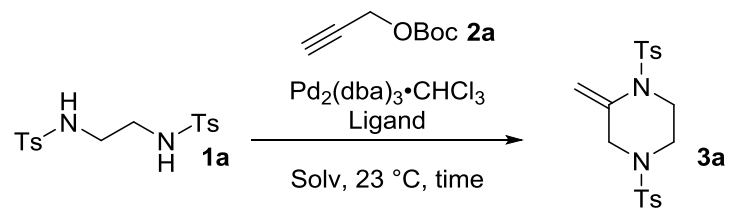

\begin{tabular}{cccccc}
\hline Entry & $\mathrm{Pd}(\mathrm{mol} \%)$ & Ligand & Solvent & Time (min) & Yield (\%) \\
\hline 1 & 5 & DPEphos & Acetone & 30 & 71 \\
2 & 5 & DPEphos & $\mathrm{CH}_{2} \mathrm{Cl}_{2}$ & 10 & $>95$ \\
3 & 5 & Xantphos & $\mathrm{CH}_{2} \mathrm{Cl}_{2}$ & 10 & $>95$ \\
4 & 5 & dppb & $\mathrm{CH}_{2} \mathrm{Cl}_{2}$ & 10 & 87 \\
5 & 5 & dppf & $\mathrm{CH}_{2} \mathrm{Cl}_{2}$ & 10 & $>95$ \\
6 & 5 & dppe & $\mathrm{CH}_{2} \mathrm{Cl}_{2}$ & 10 & $\mathrm{NR}$ \\
7 & 5 & $(R)$-BINAP & $\mathrm{CH}_{2} \mathrm{Cl}_{2}$ & 10 & $\mathrm{NR}$ \\
8 & 5 & P(2-furyl $)_{3}$ & $\mathrm{CH}_{2} \mathrm{Cl}_{2}$ & 10 & $>95$ \\
9 & 5 & P(t-Bu) ${ }_{3}$ & $\mathrm{CH}_{2} \mathrm{Cl}_{2}$ & 10 & $\mathrm{NR}$ \\
10 & 3 & DPEphos & $\mathrm{CH}_{2} \mathrm{Cl}_{2}$ & 20 & 97 \\
11 & 2 & DPEphos & $\mathrm{CH}_{2} \mathrm{Cl}_{2}$ & 110 & 90 \\
12 & 1 & DPEphos & $\mathrm{CH}_{2} \mathrm{Cl}_{2}$ & 240 & 50 \\
13 & 0.5 & DPEphos & $\mathrm{CH}_{2} \mathrm{Cl}_{2}$ & 240 & $\mathrm{NR}$
\end{tabular}

${ }^{a}[\mathrm{Pd}]\left(\mathrm{X}\right.$ mol \%), ligand (1.1X mol \%), $2 \mathrm{a}$ (1.3 equiv), $\mathrm{N}_{2}$ atmosphere, $23{ }^{\circ} \mathrm{C}, 0.1 \mathrm{M}$. ${ }^{b} \mathrm{NMR}$ yield based on internal standard. Additional $\mathrm{Pd}$ sources [e.g., $\mathrm{Pd}\left(\mathrm{PPh}_{3}\right)_{4}, \mathrm{Pd}(\mathrm{OAc})_{2}$ ] and solvents (MeCN, THF) were tried but gave inferior results.

\footnotetext{
${ }^{2}$ S. S. Zlaesskiy, V. P. Ananikov, Organometallics 2012, 31, 2302.
} 


\section{Preparation of tert-Butyl Propargyl Carbonates}

General Method: Prepared according to a modified procedure from Chalasani and coworkers $^{3}$ as we have previously described. ${ }^{1}$ To a solution of propargyl alcohol in anh. $\mathrm{CH}_{2} \mathrm{Cl}_{2}$ maintained under a positive pressure of nitrogen was added $\mathrm{N}(i-\mathrm{Pr})_{2} \mathrm{Et}$ and DMAP. The reaction mixture was cooled to $0{ }^{\circ} \mathrm{C}$ and ditert-butyl dicarbonate was either added portionwise over two min or dropwise as a solution in $\mathrm{CH}_{2} \mathrm{Cl}_{2}$. The reaction mixture was slowly warmed to ambient temperature over $3 \mathrm{~h}$. The reaction mixture was diluted with $\mathrm{CH}_{2} \mathrm{Cl}_{2}$ and washed with water, $10 \%$ aq. $\mathrm{HCl}$, sat. aq. $\mathrm{NaHCO}_{3}$, and brine. The organic layer was then dried over anh. $\mathrm{MgSO}_{4}$ and concentrated in vacuo. The residue was purified by flash column chromatography to give the desired product.

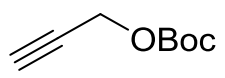

tert-butyl prop-2-yn-1-yl carbonate (2a): Prepared according to the general method using propargyl alcohol (1.7 mL, $30.0 \mathrm{mmol}), \mathrm{Boc}_{2} \mathrm{O}(8.51 \mathrm{~g}, 39 \mathrm{mmol}), \mathrm{N}(i-\operatorname{Pr})_{2} \mathrm{Et}(13.1 \mathrm{~mL}, 75 \mathrm{mmol})$, DMAP (366 mg, 3 $\mathrm{mmol}$ ), and $\mathrm{CH}_{2} \mathrm{Cl}_{2}\left(2.5 \mathrm{~mL}\right.$ ) for $16 \mathrm{~h}$. Purified by filtration over a short plug of $\mathrm{SiO}_{2}$ to afford $2 \mathrm{a}(3.33 \mathrm{~g}$, $71 \%)$ as a colorless liquid. The analytical data match those reported in the literature. ${ }^{4}$

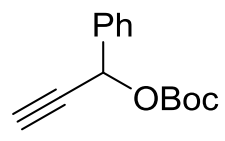

tert-butyl (1-phenylprop-2-yn-1-yl) carbonate (2b): Prepared according to the general method using 1phenylprop-2-yn-1-ol $(920 \mu \mathrm{L}, 7.57 \mathrm{mmol}), \mathrm{Boc}_{2} \mathrm{O}(2.2 \mathrm{~g}, 9.9 \mathrm{mmol}), \mathrm{N}(i-\operatorname{Pr})_{2} \mathrm{Et}(3.3 \mathrm{~mL}, 18.9 \mathrm{mmol})$, DMAP (93 mg, $0.76 \mathrm{mmol})$, and $\mathrm{CH}_{2} \mathrm{Cl}_{2}(640 \mu \mathrm{L})$. Purified by flash column chromatography (10\% EtOAc/Hexanes) to afford $\mathbf{2 b}(1.54 \mathrm{~g}, 88 \%)$ as a red/brown liquid. The analytical data match those reported in the literature. ${ }^{5}$

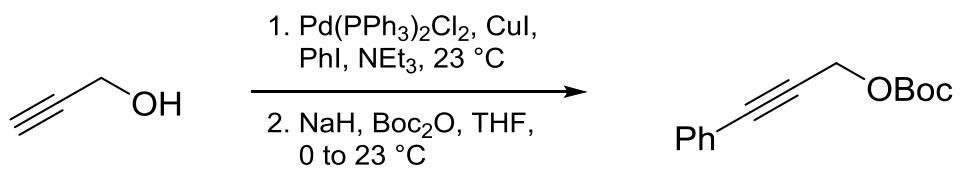

tert-butyl (3-phenylprop-2-yn-1-yl) carbonate (2c): Step 1: Prepared according to procedure by Panteleev and coworkers. ${ }^{6} \mathrm{Pd}\left(\mathrm{PPh}_{3}\right)_{2} \mathrm{Cl}_{2}(35 \mathrm{mg}, 50 \mu \mathrm{mol})$ and $\mathrm{Cul}(19 \mathrm{mg}, 0.1 \mathrm{mmol}$ ) were suspended in $\mathrm{NEt}_{3}(20 \mathrm{~mL}, 0.275 \mathrm{M})$ under a positive pressure of nitrogen. To this suspension was added iodobenzene $(560 \mu \mathrm{L}, 5.0 \mathrm{mmol})$ and propargyl alcohol $(320 \mu \mathrm{L}, 5.5 \mathrm{mmol})$. The reaction was stirred for $16 \mathrm{~h}$ at ambient temperature. Any precipitate was removed by filtration through a plug of Celite ${ }^{\mathrm{TM}}$, which was then washed with $\mathrm{NEt}_{3}(2 \times 10 \mathrm{~mL})$. The reaction mixture was passed through a plug of silica and concentrated in vacuo. This residue was used without further purification. Step 2: The crude residue was

${ }^{3}$ Chalasani, D.; Li, J.; Jackson, N. M.; Payne, M.; Lucht, B. L. J. Power Sources 2012, $15,67$.

${ }^{4}$ Štambask, J.; Malkov, A. V.; KočOVSK, P. Collect. Czech. Chem. Commun. 2008, 73, 705.

${ }^{5}$ Yamamoto, H.; Nishiyama, M.; Imagawa, H.; Nishizawa, M. Tetrahedron Lett. 2006, 47, 8369.

${ }^{6}$ Panteleev, J.; Huang, R. Y.; Lui, E. K. J.; Lautens, M. Org. Lett. 2011, 13, 5314. 
dissolved in $15 \mathrm{~mL}$ THF and added to a suspension of $60 \% \mathrm{w} / \mathrm{w} \mathrm{NaH}$ (320 mg, $8.0 \mathrm{mmol}$ ) in THF (10 mL). The solution was cooled to $0{ }^{\circ} \mathrm{C}$ and a solution of $\mathrm{Boc}_{2} \mathrm{O}(982 \mathrm{mg}, 4.5 \mathrm{mmol})$ in THF $(25 \mathrm{~mL})$ was added dropwise. Upon warming to ambient temperature the mixture was allowed to stir until starting material was consumed. The solvent was removed in vacuo and the resulting residue was dissolved in $\mathrm{CH}_{2} \mathrm{Cl}_{2}$, washed with water, which was back extracted two times with $\mathrm{CH}_{2} \mathrm{Cl}_{2}$. The combined organic layers were washed with brine, dried over $\mathrm{MgSO}_{4}$ and concentrated in vacuo. Purification by flash column chromatography (10\% EtOAc/hexanes) afforded 2c (929 mg, 73\% yield, two steps) as a red/brown liquid. The analytical data matched those reported in the literature. ${ }^{5}$

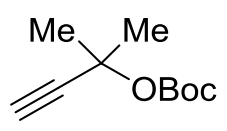

tert-butyl (2-methylbut-3-yn-2-yl) carbonate (2d): Prepared according to Štambask and coworkers. ${ }^{4}$ To a suspension of $60 \% \mathrm{w} / \mathrm{w} \mathrm{NaH}(0.62 \mathrm{~g}, 15.5 \mathrm{mmol})$ in THF $(40 \mathrm{~mL})$ maintained under a positive pressure of nitrogen was added 2-methyl-3-butyn-ol $(1.02 \mathrm{~mL}, 10.5 \mathrm{mmol})$. This mixture was allowed to stir at ambient temperature for $30 \mathrm{~min}$ and was then cooled to $0{ }^{\circ} \mathrm{C}$. To this mixture was added a solution of $\mathrm{Boc}_{2} \mathrm{O}(2.18 \mathrm{~g}, 10.0 \mathrm{mmol})$ in THF $(60 \mathrm{~mL})$ over $1 \mathrm{~h}$ by syringe pump. Following completion of the addition the mixture was allowed to warm to ambient temperature and stir for $16 \mathrm{~h}$. After consumption of the starting material, as determined by TLC, the reaction mixture was concentrated in vacuo. The residue was dissolved in $\mathrm{CH}_{2} \mathrm{Cl}_{2}$ and washed with water. The aqueous phase was extracted with $\mathrm{CH}_{2} \mathrm{Cl}_{2}$ and the combined organic layers were washed with brine. The combined organic layer was dried with anh. $\mathrm{MgSO}_{4}$, passed through a short plug of $\mathrm{SiO}_{2}$ and concentrated in vacuo to afford $\mathbf{2 d}(1.05 \mathrm{~g}, 57 \%)$ as a colorless liquid. The analytical data match those reported in the literature. $^{5}$
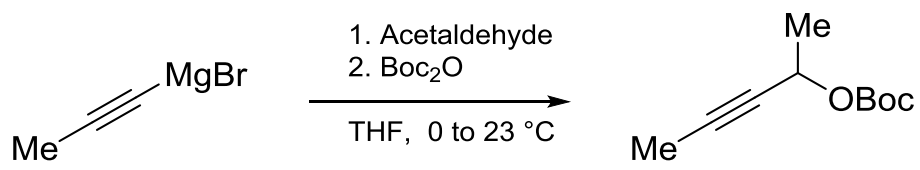

tert-butyl (pent-3-yn-2-yl) carbonate (2e): Prepared according to Tamaru and coworkers. ${ }^{7}$ To a dry round bottomed flask equipped with a stirbar under an atmosphere of nitrogen a solution of 1propynylmagnesium bromide ( $0.5 \mathrm{M}$ in THF, $10 \mathrm{mmol}$ ) was added via syringe. The solution was cooled to $0{ }^{\circ} \mathrm{C}$ and acetaldehyde $(0.85 \mathrm{~mL}, 15 \mathrm{mmol})$ was added dropwise over five minutes. The reaction mixture was allowed to warm to ambient temperature and stir for an additional $2 \mathrm{~h}$. At which time di-tert-butyl dicarbonate $(2.18 \mathrm{~g}, 10 \mathrm{mmol})$ was added to the reaction mixture and allowed to stir an additional $16 \mathrm{~h}$. The mixture was concentrated in vacuo and the residue was dissolved in $\mathrm{CH}_{2} \mathrm{Cl}_{2}$ and washed with water. This was back extracted two times with $\mathrm{CH}_{2} \mathrm{Cl}_{2}$ and the combined organic layers were washed with brine and dried over $\mathrm{MgSO}_{4}$. The solution was passed through a plug of silica and concentrated in vacuo to give $2 \mathrm{e}(1.18 \mathrm{~g}, 64 \%$ yield over two steps) as a clear oil.

\footnotetext{
${ }^{7}$ Tamaru, Y.; Kimura, M.; Tanaka, S.; Kure, S.; Yoshida, Z.-i. Bull. Chem. Soc. Jpn. 1994, 67, 2838.
} 
Analytical data for $2 \mathrm{e}:{ }^{1} \mathrm{H}$ NMR $\left(500 \mathrm{MHz}, \mathrm{CDCl}_{3}\right) \delta 5.31-5.09(\mathrm{~m}, 1 \mathrm{H}), 1.83(\mathrm{dd}, J=2.3,1.0 \mathrm{~Hz}),, 1.48(\mathrm{~s}$, $9 \mathrm{H}), 1.47$ (d, $J=6.6 \mathrm{~Hz}, 3 \mathrm{H}) ;{ }^{13} \mathrm{C}$ NMR $\left(126 \mathrm{MHz}, \mathrm{CDCl}_{3}\right) \delta 152.60,82.35,81.56,77.44,63.64,27.74,21.71$ (x3), 3.55; HRMS (ESI) calcd for $\left(\mathrm{C}_{10} \mathrm{H}_{17} \mathrm{O}_{3}\right)^{+}[\mathrm{M}+\mathrm{H}]^{+}:$185.1172, found: 185.1167 .

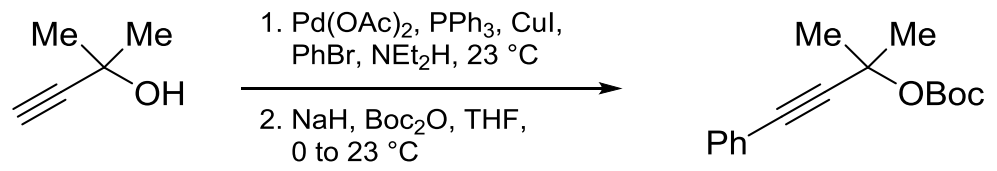

tert-butyl (2-methyl-3-phenylbut-3-yn-2-yl) carbonate (2f): Step 1: Prepared according to a procedure by $\mathrm{Li}$ and coworkers. ${ }^{8}$ To a $125-\mathrm{mL}$ flame-dried RBF was added $\mathrm{HNEt}_{2}(125 \mathrm{~mL}), \mathrm{PhBr}(7.6 \mathrm{~mL}, 60 \mathrm{mmol})$, 2-methyl-3-butyn-2-ol (4.9 mL, $50 \mathrm{mmol}$ ), $\mathrm{PPh}_{3}$ (197 mg, $\left.0.75 \mathrm{mmol}\right)$, Cul (95 mg, $\left.0.5 \mathrm{mmol}\right)$, and $\mathrm{Pd}(\mathrm{OAc})_{2}\left(56 \mathrm{mg}, 0.25 \mathrm{mmol}\right.$ ). The mixture was sparged with $\mathrm{N}_{2}$ for $30 \mathrm{~min}$, then the RBF was fitted with a reflux condenser and sparged for an additional $5 \mathrm{~min}$. The reaction was allowed to reflux for $22 \mathrm{~h}$. After cooling to ambient temperature, the mixture was concentrated in vacuo. Purified by flash column chromatography (10\% EtOAc/Hexanes) to afford the Sonogashira product $(6.98 \mathrm{~g}, 43.6 \mathrm{mmol}, 87 \%)$ as a tan solid. Analytical data match those reported in the literature.8 Step 2: The Sonogashira product (1.76 $\mathrm{g}, 11.0 \mathrm{mmol}$ ) was dissolved in THF $(45 \mathrm{~mL})$ and added to a suspension of $60 \% \mathrm{w} / \mathrm{w} \mathrm{NaH}(840 \mathrm{mg}, 21.0$ $\mathrm{mmol})$ in THF (30 mL). The solution was cooled to $0{ }^{\circ} \mathrm{C}$ and a solution of $\mathrm{Boc}_{2} \mathrm{O}(2.29 \mathrm{~g}, 10.5 \mathrm{mmol})$ in THF (35 mL) was added dropwise. Upon warming to ambient temperature the mixture was allowed to stir until starting material was consumed. The solvent was removed in vacuo and the resulting residue was dissolved in $\mathrm{CH}_{2} \mathrm{Cl}_{2}$, washed with water which was back extracted two times with $\mathrm{CH}_{2} \mathrm{Cl}_{2}$. The combined organic layers were washed with brine, dried over $\mathrm{MgSO}_{4}$ and concentrated in vacuo. Purified by flash column chromatography (10\% EtOAc/Hexanes) and the method by Base and Hassner ${ }^{9}$ (for the destruction of excess $\left.\mathrm{Boc}_{2} \mathrm{O}\right)$ to afford $2 \mathrm{f}(1.67 \mathrm{~g}, 6.4 \mathrm{mmol}, 58 \%$ yield) as an amber liquid which solidified on standing. The analytical data matched those reported in the literature. ${ }^{1}$

\section{Preparation of Bis-Sulfonamide Substrates}

General Method: Based on the protocol of Urabe and coworkers ${ }^{10}$. To a solution of diamine in anhydrous pyridine $(0.2 \mathrm{M})$ cooled to $0{ }^{\circ} \mathrm{C}$ was added sulfonyl chloride portion wise over 5 minutes. The solution was then sealed, purged three times with nitrogen and allowed to warm to ambient temperature. The reaction mixture was allowed to stir for an additional $18 \mathrm{~h}$. The solution was poured into water (six times the volume of pyridine used) and stirred for $5 \mathrm{~min}$. The formed precipitate was collected by vacuum filtration and subsequently washed two times with water and four times with diethyl ether. The solid was then collected and dried in vacuo to give the desired product.

\footnotetext{
${ }^{8}$ Li, Y.; Zou, H.; Gong, J.; Xiang, J.; Luo, T.; Quan, J.; Wang, G.; Yang, Z. Org. Lett., 2007, 9, 4057.

${ }^{9}$ Base, Y.; Hassner, A. Synthesis 2001, 550-551.

${ }^{10}$ Fukudome, Y.; Naito, H.; Hata, T.; Urabe, H. J. Am. Chem. Soc. 2008, 130, 1820.
} 


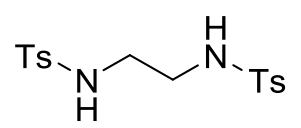

$\boldsymbol{N}, \boldsymbol{N}^{\prime}$-Bis( $\boldsymbol{p}$-toluenesulfonyl)ethylenediamine (1a): Prepared according to the general method using ethylenediamine $(0.67 \mathrm{~mL}, 10 \mathrm{mmol})$, tosyl chloride $(3.91 \mathrm{~g}, 20.5 \mathrm{mmol})$ and pyridine $(50 \mathrm{~mL})$. Purified by vacuum filtration and washing with diethyl ether to afford $1 \mathrm{a}(2.47 \mathrm{~g}, 67 \%$ yield) as an off white solid. The analytical data matched those reported in the literature. ${ }^{11}$<smiles>O=[SH](NCCNS(=O)(=O)c1ccccc1)c1ccccc1</smiles>

$\mathbf{N}, N^{\prime}$-Bis(phenylsulfonyl)ethylenediamine (1b): Prepared according to the general method using ethylenediamine $(0.10 \mathrm{~mL}, 1.5 \mathrm{mmol})$, benzenesulfonyl chloride $(0.39 \mathrm{~mL}, 3.08 \mathrm{mmol})$, and pyridine $(7.5$ $\mathrm{mL}$ ). Purified by vacuum filtration and washing with diethyl ether to afford $\mathbf{1 b}$ ( $295 \mathrm{mg}, 58 \%$ yield) as a white solid. The analytical data matched those reported in the literature. ${ }^{12}$

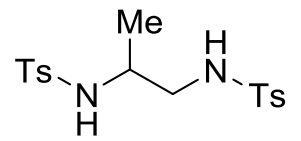

N,N'-(propane-1,2-diyl)bis(4-methylbenzenesulfonamide) (1c): Prepared according to the general method using 1,2-diaminopropane $(0.09 \mathrm{~mL}, 1.0 \mathrm{mmol})$, tosyl chloride (391 mg, $2.05 \mathrm{mmol}$ ) and pyridine $(5 \mathrm{~mL})$. Diluted with water and extracted with EtOAc (2x), the organic layer was subsequently washed with $1 \mathrm{M} \mathrm{HCl}$, brine, dried over $\mathrm{MgSO}_{4}$ and concentrated in vaccuo. Residual pyridine was removed azeotropically by dissolving the residue in toluene and removal of solvent in vacuo to afford 1c ( $222 \mathrm{mg}$, $58 \%$ yield) as an off white solid. The analytical data matched those reported in the literature. ${ }^{13}$<smiles>[3H]NC[C@H](Cc1ccccc1)N[3H]</smiles>

(S)-N,N'-(3-phenylpropane-1,2-diyl)bis(4-methylbenzenesulfonamide) (1d): Step 1: A dry round bottomed flask was charged with L-Phenylalaninamide $(500 \mathrm{mg}, 3.04 \mathrm{mmol})$, followed by addition of THF (16 mL) via syringe. The reaction flask was then purged $3 \times \mathrm{N}_{2}$ and cooled to $0{ }^{\circ} \mathrm{C}$ in an ice water bath. After 30 minutes lithium aluminum hydride (1.0 M in THF) $(6.1 \mathrm{~mL}, 6.08 \mathrm{mmol})$ was added dropwise over 15 minutes via syringe. Following the addition the reaction flask was equipped with a reflux condenser, purged $3 \times \mathrm{N}_{2}$ and heated to reflux for $12 \mathrm{~h}$. After cooling to ambient temperature residual lithium aluminum hydride was quenched using standard Fieser workup protocol: The reaction mixture was cooled to $0{ }^{\circ} \mathrm{C}$ followed by slow addition of water $(0.25 \mathrm{~mL})$. After frothing had stopped $15 \%$

\footnotetext{
${ }^{11}$ Romba, J.; Kuppert, D.; Morgenstern, B.; Neis, C.; Steinhauser, S.; Weyhermüller, T.; Hegetschweiler, K. Eur. J. Inorg. Chem. 2006, 314.

${ }^{12}$ A. Khazaei, A. Rostami, M. Mahboubifar, Catal. Commun. 2007, 8, 383.

${ }^{13}$ M. Yar, E. M. McGarrigle, V. K. Aggarwal, Angew. Chem. Int. Ed. 2008, 47, 3784.
} 
$\mathrm{NaOH}_{\text {(aq) }}(0.25 \mathrm{~mL})$ was added dropwise, followed by water $(0.75 \mathrm{~mL})$. The reaction mixture was then allowed to warm to ambient temperature and left stirring for 30 minutes. At this point anh. $\mathrm{MgSO}_{4}$ was added and the heterogeneous mixture was filtered over Celite. After removal of solvent in vacuo a yellow oil was isolated which solidified on standing. The crude NMR showed most of the material had been reduced to the diamine and was taken on without purification.

Step 2: Prepared according to the general method using crude diamine ( $396 \mathrm{mg}, 2.63 \mathrm{mmol}$ ), tosyl chloride $(1.03 \mathrm{~g}, 5.40 \mathrm{mmol})$ and pyridine $(13 \mathrm{~mL})$. Diluted with water and extracted with EtOAc $(2 \mathrm{x})$, the organic layer was subsequently washed with $1 \mathrm{M} \mathrm{HCl}$, brine dried over $\mathrm{MgSO}_{4}$ and concentrated in vaccuo. Residual pyridine was removed azeotropically by dissolving the residue in toluene and removal of solvent in vacuo to afford $\mathbf{1 d}$ (450 $\mathrm{mg}, 38 \%$ yield) as a white solid.

Analytical data for $1 \mathrm{~d}:{ }^{1} \mathrm{H} \mathrm{NMR}\left(500 \mathrm{MHz}, \mathrm{CDCl}_{3}\right) \delta 7.68(\mathrm{~d}, J=8.2 \mathrm{~Hz}, 2 \mathrm{H}), 7.50(\mathrm{~d}, J=8.2 \mathrm{~Hz}, 2 \mathrm{H}), 7.23(\mathrm{~d}$, $J=7.9 \mathrm{~Hz}, 2 \mathrm{H}), 7.17-7.03(\mathrm{~m}, 5 \mathrm{H}), 6.84(\mathrm{~d}, J=7.2 \mathrm{~Hz}, 2 \mathrm{H}), 5.60(\mathrm{t}, J=6.4 \mathrm{~Hz}, 1 \mathrm{H}), 5.36(\mathrm{~d}, J=7.2 \mathrm{~Hz}, 1 \mathrm{H})$, $3.35(\mathrm{dd}, J=11.8,5.1 \mathrm{~Hz}, 1 \mathrm{H}), 3.00(\mathrm{dd}, J=11.8,6.6 \mathrm{~Hz}, 2 \mathrm{H}), 2.73(\mathrm{dd}, J=13.9,6.9 \mathrm{~Hz}, 1 \mathrm{H}), 2.57(\mathrm{dd}, J=$ 14.0, $7.5 \mathrm{~Hz}, 1 \mathrm{H}), 2.38$ (s, 3H), 2.37 (s, 3H); 13C NMR (126 MHz, CDCl$)_{3}$ ) $143.31,143.23,136.48,136.35$, 136.28, 129.62 (x2), 129.53 (x2), 128.96 (x2), 128.45 (x2), 127.03 (x2), $126.89(x 2), 126.50$ (x2), 54.76, 46.29, 38.35, 21.37; HRMS (ESI) calcd for $\left(\mathrm{C}_{23} \mathrm{H}_{27} \mathrm{~N}_{2} \mathrm{O}_{4} \mathrm{~S}_{2}\right)^{+}[\mathrm{M}+\mathrm{H}]^{+}: 459.1407$, found: 459.1408 .

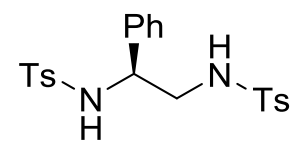

(S)-N,N'-(1-phenylethane-1,2-diyl)bis(4-methylbenzenesulfonamide) (1e): Step 1: A dry round bottomed flask was charged with (2S)-2-Amino-2-phenylethanamide (1.00 g, $6.66 \mathrm{mmol}$ ), followed by addition of THF ( $33 \mathrm{~mL}$ ) via syringe. The reaction flask was then purged $3 \times \mathrm{N}_{2}$ and cooled to $0{ }^{\circ} \mathrm{C}$ in an ice water bath. After 30 minutes lithium aluminum hydride (1.0 M in THF) (13.3 mL, $13.3 \mathrm{mmol}$ ) was added dropwise over 15 minutes via syringe. Following the addition the reaction flask was equipped with a reflux condenser, purged $3 \times \mathrm{N}_{2}$ and heated to reflux for $12 \mathrm{~h}$. After cooling to room temperature residual lithium aluminum hydride was quenched using standard Fieser workup protocol: The reaction mixture was cooled to $0{ }^{\circ} \mathrm{C}$ followed by slow addition of water $(0.5 \mathrm{~mL})$. After frothing had stopped $15 \% \mathrm{NaOH}_{\text {(aq) }}$ $(0.5 \mathrm{~mL})$ was added dropwise, followed by water $(1.5 \mathrm{~mL})$. The reaction mixture was then allowed to warm to ambient temperature and left stirring for 30 minutes. At this point anh. $\mathrm{MgSO}_{4}$ was added and the heterogeneous mixture was filtered over Celite. After removal of solvent in vacuo a yellow oil was isolated. The crude NMR showed most of the material had been reduced to the diamine and the oil was taken on without purification.

Step 2: Prepared by dissolving the crude diamine ( $739 \mathrm{mg}, 5.42 \mathrm{mmol}$ ) in $\mathrm{CH}_{2} \mathrm{Cl}_{2}$ (30 mL) and the solution was then transferred to a dry round bottomed flask and sealed under $\mathrm{N}_{2}$. The flask was cooled to $0{ }^{\circ} \mathrm{C}$ in an ice water bath and $\mathrm{NEt}_{3}(1.88 \mathrm{~mL}, 13.56 \mathrm{mmol})$ was added via syringe. Tosyl chloride $(2.12 \mathrm{~g}, 11.12$ mmol) was added as a solution in $\mathrm{CH}_{2} \mathrm{Cl}_{2}(14 \mathrm{~mL})$ and added dropwise over the course of $3 \mathrm{~h}$. The resulting mixture was allowed to warm to ambient temperature and stir for an additional $12 \mathrm{~h}$. The reaction mixture was then diluted with $\mathrm{CH}_{2} \mathrm{Cl}_{2}$ and washed with sat. $\mathrm{NaCO}_{3} \mathrm{H}$, water and brine. The organic layer was dried over $\mathrm{Na}_{2} \mathrm{SO}_{4}$ and concentrated in vacuo to give a yellow residue. The residue was 
purified by flash column chromatography (1:1 to 0:1 Hex: $\left.\mathrm{CH}_{2} \mathrm{Cl}_{2}\right)$ to afford 1 e $(981 \mathrm{mg}, 41 \%$ yield) as a pale yellow amorphous solid.

Analytical data for $1 \mathrm{e}:{ }^{1} \mathrm{H}$ NMR $\left(500 \mathrm{MHz}, \mathrm{CDCl}_{3}\right) \delta 7.62(\mathrm{~d}, J=7.9 \mathrm{~Hz}, 2 \mathrm{H}), 7.51(\mathrm{~d}, J=7.9 \mathrm{~Hz}, 2 \mathrm{H}), 7.18(\mathrm{~d}$, $J=7.9 \mathrm{~Hz}, 3 \mathrm{H}), 7.04(\mathrm{t}, J=10.5 \mathrm{~Hz}, 6 \mathrm{H}), 6.94(\mathrm{~d}, J=7.0 \mathrm{~Hz}, 2 \mathrm{H}), 4.41-4.31(\mathrm{~m}, 1 \mathrm{H}), 3.11(\mathrm{dd}, J=13.6,8.1$ $\mathrm{Hz}, 1 \mathrm{H}), 3.06(\mathrm{dd}, J=13.7,4.8 \mathrm{~Hz}, 1 \mathrm{H}), 2.33(\mathrm{~s}, 3 \mathrm{H}), 2.27(\mathrm{~s}, 3 \mathrm{H}) ;{ }^{13} \mathrm{C} \mathrm{NMR}\left(126 \mathrm{MHz}, \mathrm{CDCl}_{3}\right) \delta 143.18$, $142.89,137.37,136.75,136.39,129.45$ (x2), 129.07 (x2), 128.49 (x2), 128.21 (x2), 127.50, 126.84 (x2), 126.76 (x2), 126.47, 57.33, 47.82, 21.16; HRMS (ESI) calcd for $\left(\mathrm{C}_{22} \mathrm{H}_{25} \mathrm{~N}_{2} \mathrm{O}_{4} \mathrm{~S}_{2}\right)+[\mathrm{M}+\mathrm{H}]+:$ :445.1250, found: 445.1243.

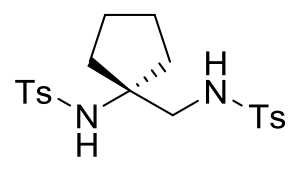

4-methyl-N-((1-((4-methylphenyl)sulfonamido)cyclopentyl)methyl)benzenesulfonamide (1f): Step 1: A dry round bottomed flask was charged with 1-aminocyclopentane-1-carboxamide (500 mg, $3.90 \mathrm{mmol}$ ), followed by addition of THF $(20 \mathrm{~mL})$ via syringe. The reaction flask was then purged $3 \times \mathrm{N}_{2}$ and cooled to $0{ }^{\circ} \mathrm{C}$ in an ice water bath. After 30 minutes lithium aluminum hydride (1.0 M in THF) ( $\left.7.8 \mathrm{~mL}, 7.80 \mathrm{mmol}\right)$ was added dropwise over 15 minutes via syringe. Following the addition the reaction flask was equipped with a reflux condenser, purged $3 \times \mathrm{N}_{2}$ and heated to reflux for $12 \mathrm{~h}$. After cooling to room temperature residual lithium aluminum hydride was quenched using standard Fieser workup protocol: The reaction mixture was cooled to $0{ }^{\circ} \mathrm{C}$ followed by slow addition of water $(0.30 \mathrm{~mL})$. After frothing had stopped $15 \%$ $\mathrm{NaOH}_{\text {(aq) }}(0.30 \mathrm{~mL})$ was added dropwise, followed by water $(0.90 \mathrm{~mL})$. The reaction mixture was then allowed to warm to ambient temperature and left stirring for 30 minutes. At this point anh. $\mathrm{MgSO}_{4}$ was added and the heterogeneous mixture was filtered over Celite. After removal of solvent in vacuo a clear oil was isolated. The crude NMR showed most of the material had been reduced to the diamine and the oil was taken on without purification.

Step 2: Prepared by dissolving the crude diamine $(256 \mathrm{mg}, 2.24 \mathrm{mmol})$ in $\mathrm{CH}_{2} \mathrm{Cl}_{2}(4 \mathrm{~mL})$ and the solution was then transferred to a dry round bottomed flask and sealed under $\mathrm{N}_{2}$. The flask was cooled to $0{ }^{\circ} \mathrm{C}$ in an ice water bath and $\mathrm{NEt}_{3}(0.78 \mathrm{~mL}, 5.60 \mathrm{mmol})$ was added via syringe. Tosyl chloride $(875 \mathrm{mg}, 4.59$ mmol) was added as a solution in $\mathrm{CH}_{2} \mathrm{Cl}_{2}(6 \mathrm{~mL})$ and added dropwise over the course of $1 \mathrm{~h}$. The resulting mixture was allowed to warm to ambient temperature and stir for an additional $12 \mathrm{~h}$. The reaction mixture was then diluted with $\mathrm{CH}_{2} \mathrm{Cl}_{2}$ and washed with water and brine. The organic layer was dried over $\mathrm{Na}_{2} \mathrm{SO}_{4}$ and concentrated in vacuo to give a residue. The residue was purified by flash column chromatography (8:1 to 3:1 Hex:EtOAc) to afford $\mathbf{1 f}$ ( $1.13 \mathrm{~g}, 51 \%$ yield) as a white amorphous solid.

Analytical data for 1f: ${ }^{1} \mathrm{H} N M R\left(500 \mathrm{MHz}, \mathrm{CDCl}_{3}\right) \delta 7.76(\mathrm{~d}, \mathrm{~J}=3.4 \mathrm{~Hz}, 2 \mathrm{H}), 7.74(\mathrm{~d}, \mathrm{~J}=3.4 \mathrm{~Hz}, 2 \mathrm{H}), 7.28$ (d, $\mathrm{J}=8.4 \mathrm{~Hz}, 2 \mathrm{H}), 7.25(\mathrm{~d}, \mathrm{~J}=8.3 \mathrm{~Hz}, 2 \mathrm{H}), 5.75(\mathrm{t}, \mathrm{J}=6.8 \mathrm{~Hz}, 1 \mathrm{H}), 5.58(\mathrm{~s}, 1 \mathrm{H}), 3.08(\mathrm{~d}, \mathrm{~J}=6.9 \mathrm{~Hz}, 2 \mathrm{H}), 2.41(\mathrm{~s}$, $3 \mathrm{H}), 2.40(\mathrm{~s}, 3 \mathrm{H}), 1.71-1.63(\mathrm{~m}, 2 \mathrm{H}), 1.61-1.54(\mathrm{~m}, 2 \mathrm{H}), 1.51-1.37(\mathrm{~m}, 4 \mathrm{H}) ;{ }^{13} \mathrm{C} \mathrm{NMR}\left(126 \mathrm{MHz}, \mathrm{CDCl}_{3}\right)$ $\delta$ 143.13, 143.07, 139.36, 137.04, 129.56 (x2), 129.52 (x2), 126.86 (x2), 126.68 (x2), 67.36, 49.60, 35.89, 31.42, $22.54(\mathrm{x} 2), 21.36,13.85$; HRMS (ESI) calcd for $\left(\mathrm{C}_{20} \mathrm{H}_{27} \mathrm{~N}_{2} \mathrm{O}_{4} \mathrm{~S}_{2}\right)+[\mathrm{M}+\mathrm{H}]+$ : 423.1407, found: 423.1400 . 
<smiles>[AlH2]C(Pc1ccccc1)C(N[As])c1ccccc1</smiles>

N,N'-((1R,2R)-1,2-diphenylethane-1,2-diyl)bis(4-methylbenzenesulfonamide) (1g): Prepared according to a protocol by Giuffredi and coworkers. ${ }^{14} \mathrm{~A}$ dry round bottomed flask was charged with $(1 R, 2 R)-(+)$ 1,2-diphenylethylenediamine $(250 \mathrm{mg}, 1.18 \mathrm{mmol})$, followed by addition of THF $(8 \mathrm{~mL})$, this was stirred under nitrogen until all solids dissolved. The reaction mixture was cooled to $0{ }^{\circ} \mathrm{C}$ and triethyl amine $(0.13$ $\mathrm{mL}, 0.9 \mathrm{mmol}$ ) was added via syringe. After stirring 1 minute tosyl chloride (245 $\mathrm{mg}, 1.28 \mathrm{mmol}$ ) was added portionwise over 2 minutes. After stirring for an additional $2 \mathrm{~h}$ the reaction mixture was diluted with $\mathrm{CH}_{2} \mathrm{Cl}_{2}$ and quenched with $\mathrm{NaCO}_{3} \mathrm{H}(\mathrm{aq})$. The aqueous layer was extracted 3 times with $\mathrm{CH}_{2} \mathrm{Cl}_{2}$, the combined organic layers were washed with brine, dried over $\mathrm{Na}_{2} \mathrm{SO}_{4}$ and concentrated in vacuo. The resulting residue was purified by flash chromatography ( $2 \% \mathrm{MeOH} / \mathrm{CH}_{2} \mathrm{Cl}_{2}$ to $5 \% \mathrm{MeOH} / \mathrm{CH}_{2} \mathrm{Cl}_{2}$ ) to afford $1 \mathrm{~g}$ (614 mg, 24\% yield) as a white solid. The analytical data matched those reported in the literature. ${ }^{15}$<smiles>[3H]N[C@@H]1CCCC[C@@H]1N[TeH]</smiles>

N,N'-((1R,2R)-cyclohexane-1,2-diyl)bis(4-methylbenzenesulfonamide) (1h): Prepared according to a protocol by Stones and coworkers. ${ }^{16} \mathrm{~A}$ dry round bottomed flask was charged with $(1 R, 2 R)-(-)-$ diaminocyclohexane $(228 \mathrm{mg}, 2.0 \mathrm{mmol})$, followed by addition of $\mathrm{CH}_{2} \mathrm{Cl}_{2}(2 \mathrm{~mL})$ and stirred until solids are dissolved. $\mathrm{N}(i \mathrm{Pr})_{2} \mathrm{Et}(2.2 \mathrm{~mL}, 12.8 \mathrm{mmol})$ was added via syringe followed by addition of tosyl chloride (782 $\mathrm{mg}, 4.1 \mathrm{mmol}$ ) all at once. The reaction mixture was then stirred for an additional $12 \mathrm{~h}$ at ambient temperature. The reaction was quenched by the addition of $10 \% \mathrm{HCl}$ and the diluted with $\mathrm{Et}_{2} \mathrm{O}$. The aqueous phase was separated and extracted $3 \times$ with $\mathrm{Et}_{2} \mathrm{O}$, the combined organic layers were washed with brine, dried over $\mathrm{MgSO}_{4}$ and concentrated in vacuo. The resulting residue expanded into a white amorphous solid ( $770 \mathrm{mg}, 1.82 \mathrm{mmol}, 91 \%$ yield) after prolonged exposure to high vacuum, affording $\mathbf{1 h}$ and required no further purification. The analytical data matched those reported in the literature. ${ }^{16}$

\footnotetext{
${ }^{14}$ Giuffredi, G. T.; Purser, S.; Sawicki, M.; Thompson, A. L.; Gouverneur, V. Tetrahedron: Asymmetry 2009, $20,910$.

${ }^{15}$ Hilgraf, R.; Pfaltz, A. Adv. Synth. Catal. 2005, 347, 61.

${ }^{16}$ Stones, G.; Tripoli, R.; McDavid, C. L.; Roux-Duplatre, K. ; Kennedy, A. R.; Sherrington, D. C.; Gibson, C. L. Organic \& Biomolecular Chemistry 2008, 6, 374.
} 
<smiles>[AsH3]NCCCN[AsH3-]</smiles>

$\mathbf{N}, N^{\prime}$-Bis( $p$-toluenesulfonyl)1,3-propylenediamine (1i): Prepared according to the general method using 1,3-propylenediamine $(0.17 \mathrm{~mL}, 2.0 \mathrm{mmol})$, tosyl chloride (782 $\mathrm{mg}, 4.1 \mathrm{mmol}$ ) and pyridine $(6.7 \mathrm{~mL})$. Purified by vacuum filtration and washing with diethyl ether afforded $\mathbf{1 i}$ (114 $\mathrm{mg}, 16 \%$ yield), an off white solid. The analytical data matched those reported in the literature. ${ }^{17}$<smiles>[3H]NCC(C)(C)CN[TeH]</smiles>

N,N'-(2,2-dimethylpropane-1,3-diyl)bis(4-methylbenzenesulfonamide) (1j): Prepared according to the general method using 2,2-dimethyl-1,3-propanediamine $(0.12 \mathrm{~mL}, 1.0 \mathrm{mmol})$, tosyl chloride (391 $\mathrm{mg}$, $2.05 \mathrm{mmol}$ ) and pyridine $(5 \mathrm{~mL})$. Isolated by extraction into EtOAc followed by subsequent washing with water to afford a yellow oil after concentration in vaccuo. The crude material was purified by flash column chromatography to give $1 \mathbf{j}(0.27 \mathrm{~g}, 66 \%$ yield) as a yellow amorphous solid.

Analytical data matched those reported in the literature. ${ }^{18}$<smiles>[As]NCCCCN[As]</smiles>

$\mathbf{N}, \boldsymbol{N}^{\prime}$-Bis(p-toluenesulfonyl)1,4-diaminobutane (1k): Prepared according to the general method using 1,4-diaminobutane $(0.20 \mathrm{~mL}, 2.0 \mathrm{mmol})$, tosyl chloride $(782 \mathrm{mg}, 4.1 \mathrm{mmol})$ and pyridine $(10 \mathrm{~mL})$. Purified by vacuum filtration and washing with diethyl ether to afford $\mathbf{1 k}(401 \mathrm{mg}, 51 \%$ yield) as a white solid. The analytical data matched those reported in the literature. ${ }^{19}$<smiles>CCOC(=O)Nc1ccccc1NC(=O)OCC</smiles>

diethyl 1,2-phenylenedicarbamate (1): Prepared according to a protocol by Conconi and coworkers. ${ }^{20} \mathrm{~A}$ dry round bottomed flask was charged with 1,2-diamnobenzene $(216 \mathrm{mg}, 2.0 \mathrm{mmol})$, THF (13 $\mathrm{mL})$ and $\mathrm{NEt}_{3}(1.11 \mathrm{~mL}, 8.0 \mathrm{mmol})$. To this solution ethyl chloroformate $(0.76 \mathrm{~mL}, 8.0 \mathrm{mmol})$ was added dropwise, following the addition, the reaction flask was fitted with a reflux condenser and heated to reflux under $\mathrm{N}_{2}$ for $1 \mathrm{~h}$. The reaction mixture was then allowed to cool to ambient temperature and the heterogeneous mixture was filtered over celite and the filtrate was washed with $\mathrm{Et}_{2} \mathrm{O}$. The combined organic solution was concentrated in vacuo to afford 11 (504 mg, $2.0 \mathrm{mmol}$, >99\% yield). The analytical data matched those reported in the literature. ${ }^{20}$

\footnotetext{
${ }^{17}$ Tan, Y.; Wang, S. J.; Wang, Y. T.; Gao, B. X.; Ba, X. W. Synth. Commun. 2010, 40, 3648.

${ }^{18}$ Burkhart, A.; Fischer, J.; Mondrzyk, A.; Ritter, H. Macromol. Chem. Phys. 2014, 215, 421.

${ }^{19}$ Khanjin, N. A.; Hesse, M. Helv. Chim. Acta 2003, 86, 2028.

${ }^{20}$ Conconi, M. T.; Marzaro, G.; Urbani, L.; Zanusso, I.; Di Liddo, R.;. Castagliuolo, I; Brun, P.; Tonus, F.; Ferrarese, A.; Guiotto, A.; Chilin, A. Eur. J. Med. Chem. 2013, 67, 373.
} 
<smiles>O=C(Nc1ccccc1)C(=O)Nc1ccccc1</smiles>

N1,N2-diphenyloxalamide (SI-1): Prepared according to a protocol by Wang and coworkers. ${ }^{21}$ To a suspension of sodium bicarbonate $(428 \mathrm{mg}, 5.1 \mathrm{mmol})$ in THF $(4.2 \mathrm{~mL})$ under nitrogen atmosphere oxalyl chloride $(0.21 \mathrm{~mL}, 2.5 \mathrm{mmol})$ was added via syringe. Aniline $(0.46 \mathrm{~mL}, 5.1 \mathrm{mmol})$ was added dropwise to the stirring suspension over 5 minutes and the reaction was allowed to stir for an additional $1 \mathrm{~h}$. The reaction was then quenched by addition of water, the formed precipitate was isolated by vacuum filtration, washed with water and dried under high vacuum for $24 \mathrm{~h}$. The procedure afforded SI-1 (433 $\mathrm{mg}, 72 \%$ yield) as a white solid and required no further purification.

Analytical data matched those reported in the literature. ${ }^{21}$

\section{Preparation of Amino Acid Based Substrates}

General Method: Step 1: Based on the protocol by Ajani and coworkers. ${ }^{22}$ To a flask charged with amino acid (1.0 equiv.) and $\mathrm{NaHCO}_{3}(2.1$ equiv.) water $(0.8 \mathrm{M})$ is added and the suspension is set stirring at a high rate. The suspension is then cooled to $0{ }^{\circ} \mathrm{C}$ and after $20 \mathrm{~min}$ sulfonyl chloride (1.2 equiv.) is added portion-wise over five minutes. The reaction vessel is then sealed under nitrogen and allowed to slowly warm to ambient temperature. The mixture is then stirred for 4 to $18 \mathrm{~h}$ after which it is carefully quenched with $10 \% \mathrm{HCl}$. Upon acidification the formed precipitate is isolated by vacuum filtration and washed with $2.2 \mathrm{pH}$ buffer solution $(746 \mathrm{mg} \mathrm{KCl}, 1.56 \mathrm{~mL} 10 \% \mathrm{HCl}$ in $50 \mathrm{~mL}$ water). Unless otherwise indicated the crude material is taken on without further purification.

Step 2: Based on the protocol by Aguilar-Castro and coworkers. ${ }^{23}$ The crude product isolated previously was dissolved in THF (0.1 M), cooled to $0{ }^{\circ} \mathrm{C}$ and sealed under nitrogen. To this $\mathrm{SOCl}_{2}$ (2.32 equiv.) was added dropwise over five minutes and then the solution was allowed warm to ambient temperature and stirred for $1 \mathrm{~h}$. The reaction mixture was then concentrated in vacuo to remove excess $\mathrm{SOCl}_{2}$, the resulting residue was redissolved in THF $(0.1 \mathrm{M})$ and aniline (1.1 equiv.) was added dropwise over 5 minutes. The reaction mixture was allowed to stir at ambient temperature until starting material was consumed as determined by TLC. The reaction mixture was then diluted with EtOAc and water. The aqueous layer was extracted three times with EtOAc and the combined organic layers were then washed with $\mathrm{NaHCO}_{3}(\mathrm{aq})$, brine, dried over $\mathrm{MgSO}_{4}$ and concentrated in vacuo. The resulting residue was purified by flash column chromatography to give the desired compounds.

\footnotetext{
${ }^{21}$ Cotton, F. A.; Liu, C. Y.; Murillo, C. A.; Villagrán, D.; Wang, X. J. Am. Chem. Soc. 2003, 125, 13564.

${ }^{22}$ Ajani, O. O.; Familoni, O. B.; Wu, F.; Echeme, J. O.; Sujiang, Z. International Journal of Drug Design and Discovery, 2013, 4, 983.

${ }^{23}$ Aguilar-Castro, L.; Tlahuextl, M.; Tapia-Benavides, A. R.; Tlahuext, H. Heteroat. Chem 2003, $14,247$.
} 
<smiles>O=C(CNc1ccccc1)Nc1ccccc1</smiles>

2-((4-methylphenyl)sulfonamido)-N-phenylacetamide (5a): Prepared according to the general method using glycine ( $375 \mathrm{mg}, 5.0 \mathrm{mmol})$, tosyl chloride $(1.14 \mathrm{~g}, 6.0 \mathrm{mmol}), \mathrm{NaHCO}_{3}(1.11 \mathrm{~g}, 10.5 \mathrm{mmol})$ and water $(6.2 \mathrm{~mL})$ for $16 \mathrm{~h}$. A portion of the crude material was taken on to the next step. The crude solid (280 mg), SOCl 2 (0.21 mL, $2.83 \mathrm{mmol})$, aniline $(0.12 \mathrm{~mL}, 1.34 \mathrm{mmol})$ and THF (12 mL) were stirred for $3 \mathrm{~h}$ at ambient temperature. Purified by flash column chromatography (9:1 to 4:1 Hexanes:EtOAc) to afford 5a (98.4 mg, $0.32 \mathrm{mmol}, 26 \%$ yield over two steps) as a white solid.

Analytical data for $5 \mathrm{a}:{ }^{1} \mathrm{H}$ NMR $(500 \mathrm{MHz}$, Acetone) $\delta 9.17(\mathrm{~s}, 1 \mathrm{H}), 7.79(\mathrm{~d}, J=8.2 \mathrm{~Hz}, 2 \mathrm{H}), 7.57(\mathrm{~d}, J=8.0$ $\mathrm{Hz}, 2 \mathrm{H}), 7.37(\mathrm{~d}, J=8.2 \mathrm{~Hz}, 2 \mathrm{H}), 7.32-7.23(\mathrm{~m}, 2 \mathrm{H}), 7.06(\mathrm{t}, J=7.4 \mathrm{~Hz}, 1 \mathrm{H}), 6.79(\mathrm{~s}, 1 \mathrm{H}), 3.76(\mathrm{~s}, 2 \mathrm{H}), 2.38$ (s, 3H); ${ }^{13} \mathrm{C}$ NMR (126 MHz, Acetone) $\delta$ 167.09, 144.36, 139.48, 130.57 (x2), 129.61 (x2), 128.11 (x2), 124.70 (x2), 120.43 (x2), 47.20, 21.45; HRMS (ESI) calcd for $\left(\mathrm{C}_{15} \mathrm{H}_{17} \mathrm{~N}_{2} \mathrm{O}_{3} \mathrm{~S}\right)^{+}[\mathrm{M}+\mathrm{H}]^{+}: 305.0954$, found: 305.0960 .<smiles>O=C(CN[13F])Nc1ccc(Cl)cc1</smiles>

N-(4-chlorophenyl)-2-((4-methylphenyl)sulfonamido)acetamide (5b): Prepared according to the general method using glycine $(375 \mathrm{mg}, 5.0 \mathrm{mmol})$, tosyl chloride $(1.14 \mathrm{~g}, 6.0 \mathrm{mmol}), \mathrm{NaHCO}_{3}(1.11 \mathrm{~g}$, $10.5 \mathrm{mmol}$ ) and water $(6.2 \mathrm{~mL})$ for $16 \mathrm{~h}$. A portion of the crude material was taken on to the next step. The crude solid ( $280 \mathrm{mg}), \mathrm{SOCl}_{2}(0.21 \mathrm{~mL}, 2.83 \mathrm{mmol})$, 4-chloroaniline $(0.12 \mathrm{~mL}, 1.34 \mathrm{mmol}$ ) and THF (12 $\mathrm{mL}$ ) were stirred for $16 \mathrm{~h}$ at ambient temperature. Purified by flash column chromatography (9:1 to 4:1 Hexanes:EtOAc) to afford $\mathbf{5 b}(74.2 \mathrm{mg}, 0.32 \mathrm{mmol}, 18 \%$ yield over two steps) as a white solid.

Analytical data for $5 \mathbf{b}:{ }^{1} \mathrm{H}$ NMR $(500 \mathrm{MHz}$, Acetone) $\delta 9.31(\mathrm{~s}, 1 \mathrm{H}), 7.78(\mathrm{~d}, J=8.2 \mathrm{~Hz}, 2 \mathrm{H}), 7.60(\mathrm{~d}, J=8.9$ $\mathrm{Hz}, 2 \mathrm{H}), 7.41-7.34(\mathrm{~m}, 2 \mathrm{H}), 7.31(\mathrm{~d}, J=8.9 \mathrm{~Hz}, 2 \mathrm{H}), 6.82(\mathrm{~s}, 1 \mathrm{H}), 3.76(\mathrm{~s}, 2 \mathrm{H}), 2.38(\mathrm{~s}, 3 \mathrm{H}) ;{ }^{13} \mathrm{C} \mathrm{NMR}(126$ $\mathrm{MHz}$, Acetone) $\delta 168.05,145.05,139.08,131.24$ (x3), 130.21 (x3), 128.78 (x3), 122.63, 47.93, 22.11; HRMS (ESI) calcd for $\left(\mathrm{C}_{15} \mathrm{H}_{16} \mathrm{CIN}_{2} \mathrm{O}_{3} \mathrm{~S}\right)^{+}[\mathrm{M}+\mathrm{H}]^{+}: 339.0565$, found: 339.0558 .<smiles>O=C(Nc1ccccc1)[C@H](Cc1ccccc1)NC(F)(F)F</smiles>

(R)-2-((4-methylphenyl)sulfonamido)- $\mathbf{N}, 3-$ diphenylpropanamide (5c): Prepared according to the general method using $(D)$-phenylalanine $(826 \mathrm{mg}, 5.0 \mathrm{mmol})$, tosyl chloride $(1.14 \mathrm{~g}, 6.0 \mathrm{mmol}), \mathrm{NaHCO}_{3}$ $(1.11 \mathrm{~g}, 10.5 \mathrm{mmol})$ and water $(6.0 \mathrm{~mL})$ for $16 \mathrm{~h}$. A portion of the crude material was taken on to the next step. The crude solid $(723 \mathrm{mg}), \mathrm{SOCl}_{2}(0.38 \mathrm{~mL}, 5.25 \mathrm{mmol})$, aniline $(0.23 \mathrm{~mL}, 2.49 \mathrm{mmol})$ and THF $(23 \mathrm{~mL}$ ) were stirred for $16 \mathrm{~h}$ at ambient temperature. Purified by flash column chromatography (9:1 to 4:1 Hexanes:EtOAc) to afford 5c (475 mg, $1.19 \mathrm{mmol}, 53 \%$ yield over two steps) as a fluffy white solid. 
Analytical data matched those reported in the literature. ${ }^{24}$<smiles>[CH]C(=O)N[C@@H](C)C(=O)Nc1ccccc1</smiles>

(S)-2-((4-methylphenyl)sulfonamido)-N-phenylpropanamide (5d): Prepared according to the general method using (L)- alanine (445 mg, $5.0 \mathrm{mmol})$, tosyl chloride $(1.14 \mathrm{~g}, 6.0 \mathrm{mmol}), \mathrm{K}_{2} \mathrm{CO}_{3}(1.45 \mathrm{~g}, 10.5$ $\mathrm{mmol})$ and water $(6.0 \mathrm{~mL})$ for $16 \mathrm{~h}$. A portion of the crude material was taken on to the next step. The crude solid $(105 \mathrm{mg}), \mathrm{SOCl}_{2}(0.08 \mathrm{~mL}, 1.0 \mathrm{mmol})$, aniline $(0.05 \mathrm{~mL}, 0.47 \mathrm{mmol})$ and THF $(1.2 \mathrm{~mL})$ were stirred for $16 \mathrm{~h}$ at ambient temperature. Purified by flash column chromatography (9:1 to 5:2 Hexanes:EtOAc) to afford $\mathbf{5 d}$ (104 $\mathrm{mg}, 0.33 \mathrm{mmol}, 75 \%$ yield over two steps) as a white solid.

Analytical data for $5 \mathrm{~d}:{ }^{1} \mathrm{H}$ NMR $(500 \mathrm{MHz}$, Acetone) $\delta 9.11(\mathrm{~s}, 1 \mathrm{H}), 7.76(\mathrm{~d}, J=8.2 \mathrm{~Hz}, 2 \mathrm{H}), 7.50$ (d, $J=8.4$ $\mathrm{Hz}, 2 \mathrm{H}), 7.33-7.22(\mathrm{~m}, 4 \mathrm{H}), 7.06(\mathrm{t}, J=7.4 \mathrm{~Hz}, 1 \mathrm{H}), 6.75(\mathrm{~s}, 1 \mathrm{H}), 4.00(\mathrm{dd}, J=14.1,7.0 \mathrm{~Hz}, 1 \mathrm{H}), 2.31(\mathrm{~s}$, $3 \mathrm{H}), 1.29$ (d, $J=7.1 \mathrm{~Hz}, 3 \mathrm{H}) ;{ }^{13} \mathrm{C}$ NMR $(126 \mathrm{MHz}$, Acetone) $\delta 170.65,144.18,140.16,139.50,130.40$ (x2), 129.46 (x2), 128.00 (x2), 124.59 (x2), 120.40, 54.05, 21.34, 19.56; HRMS (ESI) calcd for $\left(\mathrm{C}_{16} \mathrm{H}_{19} \mathrm{~N}_{2} \mathrm{O}_{3} \mathrm{~S}\right)^{+}$ $[\mathrm{M}+\mathrm{H}]^{+}: 319.1111$, found: 319.1116.<smiles>C[C@H](NC(=O)[I-])C(=O)Nc1ccc(Cl)cc1</smiles>

(S)-N-(4-chlorophenyl)-2-((4-methylphenyl)sulfonamido)propanamide (5e): Prepared according to the general method using (L)- alanine $(891 \mathrm{mg}, 10.0 \mathrm{mmol})$, tosyl chloride $(2.10 \mathrm{~g}, 11.0 \mathrm{mmol}), \mathrm{K}_{2} \mathrm{CO}_{3}(2.90 \mathrm{~g}$, $21.0 \mathrm{mmol})$ and water $(12.0 \mathrm{~mL})$ for $16 \mathrm{~h}$. A portion of the crude material was taken on to the next step. The crude solid (122 mg), SOCl $(0.08 \mathrm{~mL}, 1.16 \mathrm{mmol})$, 4-chloroaniline (70 mg, $0.55 \mathrm{mmol}$ ) and THF (5 $\mathrm{mL}$ ) were stirred for $16 \mathrm{~h}$ at ambient temperature. Purified by flash column chromatography (9:1 to 5:2 Hexanes:EtOAc) to afford 5 e ( $38 \mathrm{mg}, 0.11 \mathrm{mmol}, 22 \%$ yield over two steps) as a white solid.

Analytical data for $5 e:{ }^{1} \mathrm{H} \mathrm{NMR}\left(500 \mathrm{MHz}, \mathrm{CDCl}_{3}\right) \delta 8.27(\mathrm{~s}, 1 \mathrm{H}), 7.77(\mathrm{~d}, J=8.3 \mathrm{~Hz}, 2 \mathrm{H}), 7.39(\mathrm{~d}, J=8.8 \mathrm{~Hz}$, $2 \mathrm{H}$ ), 7.30 (dd, $J=8.0,0.5 \mathrm{~Hz}, 2 \mathrm{H}), 7.25(\mathrm{~d}, J=9.2 \mathrm{~Hz}, 2 \mathrm{H}), 5.19(\mathrm{~d}, J=7.5 \mathrm{~Hz}, 1 \mathrm{H}), 3.91(\mathrm{t}, J=7.2 \mathrm{~Hz}, 1 \mathrm{H}$ ), $2.40(\mathrm{~s}, 3 \mathrm{H}), 1.30(\mathrm{~d}, J=7.1 \mathrm{~Hz}, 3 \mathrm{H}) ;{ }^{13} \mathrm{C}$ NMR $\left(126 \mathrm{MHz} \mathrm{CDCl}_{3}\right) \delta 169.20,144.46,135.67,130.06$ (x2), $129.61(\mathrm{x} 2), 128.96(\mathrm{x} 2), 127.26(\mathrm{x} 2), 121.19(\mathrm{x} 2), 53.27,21.52,18.56$; HRMS (ESI) calcd for $\left(\mathrm{C}_{16} \mathrm{H}_{18} \mathrm{ClN}_{2} \mathrm{O}_{3} \mathrm{~S}\right)^{+}[\mathrm{M}+\mathrm{H}]^{+}: 353.0721$, found: 353.0723 .<smiles>C[C@H](NC(=O)Nc1ccc([N+](=O)[O-])cc1)C(=O)[Hg]</smiles>

\footnotetext{
${ }^{24}$ Gou, S.; Judeh, Z. M. A. Chirality 2011, 23, 105.
} 
(S)-2-((4-methylphenyl)sulfonamido)-N-(4-nitrophenyl)propanamide (5f): Prepared according to the general method using (L)- alanine $(445 \mathrm{mg}, 5.0 \mathrm{mmol})$, tosyl chloride $(1.14 \mathrm{~g}, 6.0 \mathrm{mmol}), \mathrm{K}_{2} \mathrm{CO}_{3}(1.45 \mathrm{~g}$, $10.5 \mathrm{mmol}$ ) and water $(6.0 \mathrm{~mL})$ for $16 \mathrm{~h}$. A portion of the crude material was taken on to the next step. The crude solid $(105 \mathrm{mg}), \mathrm{SOCl}_{2}(0.08 \mathrm{~mL}, 1.0 \mathrm{mmol})$, 4-nitroaniline $(69 \mathrm{mg}, 0.47 \mathrm{mmol})$ and THF (1.2 mL) were stirred for $16 \mathrm{~h}$ at ambient temperature. Purified by flash column chromatography (9:1 to 5:2 Hexanes:EtOAc) to afford $\mathbf{5 f}$ ( $98 \mathrm{mg}, 0.27 \mathrm{mmol}, 63 \%$ yield over two steps) as a white solid.

Analytical data for $\mathbf{5 f}:{ }^{1} \mathrm{H}$ NMR $(500 \mathrm{MHz}$, Acetone) $\delta 9.68(\mathrm{~s}, 1 \mathrm{H}), 8.20(\mathrm{~d}, J=9.2 \mathrm{~Hz}, 2 \mathrm{H}), 7.79$ (d, $J=9.3$ $\mathrm{Hz}, 2 \mathrm{H}), 7.75(\mathrm{~d}, J=8.2 \mathrm{~Hz}, 2 \mathrm{H}), 7.31(\mathrm{~d}, J=8.5 \mathrm{~Hz}, 2 \mathrm{H}), 6.91(\mathrm{~d}, J=8.1 \mathrm{~Hz}, 1 \mathrm{H}), 4.13-3.99(\mathrm{~m}, 1 \mathrm{H}), 2.30$ (s, 3H), 1.32 (d, J = 7.1 Hz, 3H); ${ }^{13} \mathrm{C}$ NMR (126 MHz, Acetone) $\delta$ 171.68, 145.42, 144.32, 138.79, 130.46 (x3), 128.06 (x2), 125.47 (x2), 120.15 (x2), 54.34, 21.33, 19.16; HRMS (ESI) calcd for $\left(\mathrm{C}_{16} \mathrm{H}_{18} \mathrm{~N}_{3} \mathrm{O}_{5} \mathrm{~S}\right)^{+}$ $[\mathrm{M}+\mathrm{H}]^{+}: 364.0962$, found: 364.0958 .<smiles>C[C@H](NC(=O)Nc1cc(C(F)(F)F)cc(C(F)(F)F)c1)C(F)(F)F</smiles>

(S)-N-(3,5-bis(trifluoromethyl)phenyl)-2-((4-methylphenyl)sulfonamido)propanamide (5g): Prepared according to the general method using (L)- alanine ( $445 \mathrm{mg}, 5.0 \mathrm{mmol})$, tosyl chloride $(1.14 \mathrm{~g}, 6.0 \mathrm{mmol})$, $\mathrm{K}_{2} \mathrm{CO}_{3}(1.45 \mathrm{~g}, 10.5 \mathrm{mmol})$ and water $(6.0 \mathrm{~mL})$ for $16 \mathrm{~h}$. A portion of the crude material was taken on to the next step. The crude solid $(105 \mathrm{mg}), \mathrm{SOCl}_{2}(0.08 \mathrm{~mL}, 1.0 \mathrm{mmol}), 3,5$-bis(trifluoromethyl)aniline $(0.08$ $\mathrm{mL}, 0.47 \mathrm{mmol}$ ) and THF (1.2 mL) were stirred for $16 \mathrm{~h}$ at ambient temperature. Purified by flash column chromatography (9:1 to 5:2 Hexanes:EtOAc) to afford $\mathbf{5 g}$ (54 mg, $0.54 \mathrm{mmol}, 28 \%$ yield over two steps) as a white solid.

Analytical data for $5 \mathrm{~g}:{ }^{1} \mathrm{H}$ NMR $\left(500 \mathrm{MHz}, \mathrm{CDCl}_{3}\right) \delta 8.73(\mathrm{~s}, 1 \mathrm{H}), 7.98(\mathrm{~s}, 2 \mathrm{H}), 7.79(\mathrm{~d}, J=8.2 \mathrm{~Hz}, 2 \mathrm{H}), 7.60$ $(\mathrm{s}, 1 \mathrm{H}), 7.34(\mathrm{~d}, J=8.2 \mathrm{~Hz}, 2 \mathrm{H}), 5.21(\mathrm{~d}, J=7.1 \mathrm{~Hz}, 1 \mathrm{H}), 3.96(\mathrm{dd}, J=14.1,7.0 \mathrm{~Hz}, 1 \mathrm{H}), 2.41(\mathrm{~s}, 3 \mathrm{H}), 1.32$ (d, $J=7.1 \mathrm{~Hz}, 3 \mathrm{H}) . ;{ }^{13} \mathrm{C} \mathrm{NMR}\left(126 \mathrm{MHz}, \mathrm{CDCl}_{3}\right) \delta 170.17,169.56,145.07,138.70,130.19(x 3), 127.29$ (x4), $122.14,119.64,53.32$ (x2), 29.69, 21.47, 18.19; HRMS (ESI) calcd for $\left(\mathrm{C}_{18} \mathrm{H}_{17} \mathrm{~F}_{6} \mathrm{~N}_{2} \mathrm{O}_{3} \mathrm{~S}\right)^{+}[\mathrm{M}+\mathrm{H}]^{+}: 455.0859$, found: 455.0854 .<smiles>[3H]N[C@@H](C)C(=O)Nc1cc(C)cc(C)c1</smiles>

(S)-N-(3,5-dimethylphenyl)-2-((4-methylphenyl)sulfonamido)propanamide (5h): Prepared according to the general method using (L)- alanine $(445 \mathrm{mg}, 5.0 \mathrm{mmol})$, tosyl chloride $(1.14 \mathrm{~g}, 6.0 \mathrm{mmol}), \mathrm{K}_{2} \mathrm{CO}_{3}(1.45$ $\mathrm{g}, 10.5 \mathrm{mmol})$ and water $(6.0 \mathrm{~mL})$ for $16 \mathrm{~h}$. A portion of the crude material was taken on to the next step. The crude solid (105 mg), SOCl $(0.08 \mathrm{~mL}, 1.0 \mathrm{mmol}), 3,5$-bis(methyl)aniline $(0.06 \mathrm{~mL}, 0.47 \mathrm{mmol}$ ) and THF $(1.2 \mathrm{~mL})$ were stirred for $16 \mathrm{~h}$ at ambient temperature. Purified by flash column chromatography (9:1 to 5:2 Hexanes:EtOAc) to afford $5 \mathbf{h}$ ( $50 \mathrm{mg}, 0.14 \mathrm{mmol}, 34 \%$ yield over two steps) as a white solid. 
Analytical data for $5 \mathrm{~h}:{ }^{1} \mathrm{H}$ NMR $(500 \mathrm{MHz}$, Acetone) $\delta 8.93(\mathrm{~s}, 1 \mathrm{H}), 7.76(\mathrm{~d}, J=7.9 \mathrm{~Hz}, 2 \mathrm{H}), 7.33(\mathrm{~d}, J=7.7$ $\mathrm{Hz}, 2 \mathrm{H}), 7.11(\mathrm{~s}, 2 \mathrm{H}), 6.77(\mathrm{~s}, 1 \mathrm{H}), 6.71(\mathrm{~s}, 1 \mathrm{H}), 3.98(\mathrm{~s}, 1 \mathrm{H}), 2.33(\mathrm{~s}, 3 \mathrm{H}), 2.23(\mathrm{~s}, 6 \mathrm{H}), 1.28(\mathrm{~d}, J=6.9 \mathrm{~Hz}$, $3 \mathrm{H}) ;{ }^{13} \mathrm{C}$ NMR (126 MHz, Acetone) $\delta$ 170.47, 144.17, 139.29, 138.94, 138.92, 130.42 (x2), 128.00 (x3), $126.20(x 2), 118.24(x 2), 54.05,21.41,21.36,19.58$; HRMS (ESI) calcd for $\left(\mathrm{C}_{18} \mathrm{H}_{23} \mathrm{~N}_{2} \mathrm{O}_{3} \mathrm{~S}\right)^{+}[\mathrm{M}+\mathrm{H}]^{+}$: .347 .1424 , found: 347.1430 .<smiles>COc1ccc(NC(=O)C(C)NC(F)(F)F)cc1</smiles>

(S)-N-(4-methoxyphenyl)-2-((4-methylphenyl)sulfonamido)propanamide (5i): Prepared according to the general method using (L)- alanine $(891 \mathrm{mg}, 10.0 \mathrm{mmol})$, tosyl chloride $(2.10 \mathrm{~g}, 11.0 \mathrm{mmol}), \mathrm{K}_{2} \mathrm{CO}_{3}$ $(2.90 \mathrm{~g}, 21.0 \mathrm{mmol})$ and water $(12.0 \mathrm{~mL})$ for $16 \mathrm{~h}$. A portion of the crude material was taken on to the next step. The crude solid (122 mg), SOCl $(0.08 \mathrm{~mL}, 1.16 \mathrm{mmol}), \mathrm{p}$-anisidine $(68 \mathrm{mg}, 0.55 \mathrm{mmol})$ and THF $(5 \mathrm{~mL})$ were stirred for $16 \mathrm{~h}$ at ambient temperature. Purified by flash column chromatography (9:1 to 5:2 Hexanes:EtOAc) to afford $\mathbf{5 i}$ ( $38 \mathrm{mg}, 0.11 \mathrm{mmol}, 22 \%$ yield over two steps) as a white solid.

Analytical data for $5 \mathrm{i}:{ }^{1} \mathrm{H}$ NMR $\left(500 \mathrm{MHz}, \mathrm{CDCl}_{3}\right) \delta 8.15(\mathrm{~s}, 1 \mathrm{H}), 7.76(\mathrm{~d}, J=8.3 \mathrm{~Hz}, 2 \mathrm{H}), 7.28(\mathrm{t}, J=7.5 \mathrm{~Hz}$, $4 \mathrm{H}), 6.80(\mathrm{~d}, J=8.9 \mathrm{~Hz}, 2 \mathrm{H}), 5.52(\mathrm{~d}, J=7.7 \mathrm{~Hz}, 1 \mathrm{H}), 3.93(\mathrm{~d}, J=7.2 \mathrm{~Hz}, 1 \mathrm{H}), 3.77(\mathrm{~s}, 3 \mathrm{H}), 2.38(\mathrm{~s}, 3 \mathrm{H}), 1.30$ $(\mathrm{d}, J=7.1 \mathrm{~Hz}, 3 \mathrm{H}) ;{ }^{13} \mathrm{C}$ NMR $\left(126 \mathrm{MHz}, \mathrm{CDCl}_{3}\right) \delta$ 168.97, 156.47, 144.04, 129.94 (x2), 127.21 (x3), 121.85 (x2), $114.03(x 2), 55.68,55.42,53.17,21.63,18.86$; HRMS (ESI) calcd for $\left(\mathrm{C}_{17} \mathrm{H}_{21} \mathrm{~N}_{2} \mathrm{O}_{4} \mathrm{~S}\right)^{+}[\mathrm{M}+\mathrm{H}]^{+}$: 349.1217, found: 349.1222 .<smiles>CC(C)[C@H](NC(=O)[Na])C(=O)Nc1ccc([N+](=O)[O-])cc1</smiles>

(S)-3-methyl-2-((4-methylphenyl)sulfonamido)-N-(4-nitrophenyl)butanamide (5j): Prepared according to the general method using (L)- valine $\left(469 \mathrm{mg}, 4.0 \mathrm{mmol}\right.$ ), tosyl chloride ( $839 \mathrm{mg}, 4.4 \mathrm{mmol}$ ), $\mathrm{K}_{2} \mathrm{CO}_{3}$ $(1.16 \mathrm{~g}, 8.4 \mathrm{mmol})$ and water $(4.8 \mathrm{~mL})$ for $16 \mathrm{~h}$. A portion of the crude material was taken on to the next step. The crude solid $(250 \mathrm{mg}), \mathrm{SOCl}_{2}(0.15 \mathrm{~mL}, 2.07 \mathrm{mmol})$, 4-nitroaniline (140 mg, $1.0 \mathrm{mmol}$ ) and THF $(5 \mathrm{~mL})$ were stirred for $16 \mathrm{~h}$ at ambient temperature. Purified by flash column chromatography (9:1 to 5:2 Hexanes:EtOAc) to afford $5 \mathbf{j}$ ( $161 \mathrm{mg}, 0.41 \mathrm{mmol}, 45 \%$ yield over two steps) as a white solid.

Analytical data for $5 \mathrm{j}:{ }^{1} \mathrm{H}$ NMR $(500 \mathrm{MHz}$, Acetone) $\delta 9.63(\mathrm{~s}, 1 \mathrm{H}), 8.18(\mathrm{~d}, J=9.2 \mathrm{~Hz}, 2 \mathrm{H}), 7.78-7.62(\mathrm{~m}$, $4 \mathrm{H}), 7.33-7.13(\mathrm{~m}, 2 \mathrm{H}), 6.67(\mathrm{~d}, J=9.0 \mathrm{~Hz}, 1 \mathrm{H}), 3.76(\mathrm{dd}, J=9.0,7.0 \mathrm{~Hz}, 1 \mathrm{H}), 2.18(\mathrm{~s}, 3 \mathrm{H}), 0.96(\mathrm{dd}, J=$ 10.9, $6.8 \mathrm{~Hz}, 7 \mathrm{H}) ;{ }^{13} \mathrm{C}$ NMR (126 MHz, Acetone) $\delta$ 170.82, 145.14, 144.07, 130.22 (x3), 128.12 (x3), 125.43 (x2), $120.04(x 2), 64.06,32.31,21.22,19.48,18.39$; HRMS (ESI) calcd for $\left(\mathrm{C}_{18} \mathrm{H}_{22} \mathrm{~N}_{3} \mathrm{O}_{5} \mathrm{~S}\right)^{+}[\mathrm{M}+\mathrm{H}]^{+}$: 392.1275, found: 392.1264 . 


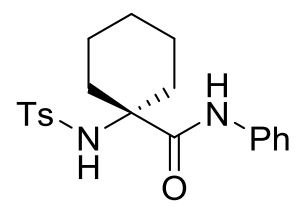

1-((4-methylphenyl)sulfonamido)-N-phenylcyclohexane-1-carboxamide (5k): Prepared according to the general method using 1-aminocyclohexanecarboxylic acid (573 mg, $4.0 \mathrm{mmol}$ ), tosyl chloride (839 mg, $4.4 \mathrm{mmol}), \mathrm{K}_{2} \mathrm{CO}_{3}(1.16 \mathrm{~g}, 8.4 \mathrm{mmol})$ and water $(4.8 \mathrm{~mL})$ for $16 \mathrm{~h}$. A portion of the crude material was taken on to the next step. The crude solid $(193 \mathrm{mg}), \mathrm{SOCl}_{2}(0.11 \mathrm{~mL}, 1.51 \mathrm{mmol})$, aniline $(0.07 \mathrm{~mL}, 0.72$ $\mathrm{mmol}$ ) and THF $(6.5 \mathrm{~mL})$ were stirred for $16 \mathrm{~h}$ at ambient temperature. Purified by flash column chromatography (9:1 to 5:2 Hexanes:EtOAc) to afford 5k (131 mg, $0.35 \mathrm{mmol}, 54 \%$ yield over two steps) as a white amorphous solid.

Analytical data for $5 \mathrm{k}^{1}{ }^{1} \mathrm{H}$ NMR $\left(500 \mathrm{MHz}, \mathrm{CDCl}_{3}\right) \delta 8.27(\mathrm{~s}, 1 \mathrm{H}), 7.78(\mathrm{~d}, \mathrm{~J}=8.3 \mathrm{~Hz}, 2 \mathrm{H}), 7.41(\mathrm{~d}, \mathrm{~J}=7.6 \mathrm{~Hz}$, 2H), $7.23(\mathrm{t}, \mathrm{J}=7.9 \mathrm{~Hz}, 2 \mathrm{H}), 7.17(\mathrm{~d}, \mathrm{~J}=8.2 \mathrm{~Hz}, 2 \mathrm{H}), 7.05(\mathrm{t}, \mathrm{J}=7.4 \mathrm{~Hz}, 1 \mathrm{H}), 5.96(\mathrm{~s}, 1 \mathrm{H}), 2.32(\mathrm{~s}, 3 \mathrm{H}), 1.96$ $(\mathrm{s}, 4 \mathrm{H}), 1.49-1.38(\mathrm{~m}, 3 \mathrm{H}), 1.31-1.19(\mathrm{~m}, 3 \mathrm{H}) ; \mathrm{C}^{13} \mathrm{NMR}\left(126 \mathrm{MHz}, \mathrm{CDCl}_{3}\right) \delta 172.00,143.53,138.76$, 137.74, 129.59 (x2), 128.96, 128.62 (x2), 128.15, 126.91 (x2), 124.19, 120.29 (x2), 63.25, 33.25, 24.86, 21.39, 21.27; HRMS (ESI) calcd for $\left(\mathrm{C}_{20} \mathrm{H}_{25} \mathrm{~N}_{2} \mathrm{O}_{3} \mathrm{~S}\right)^{+}[\mathrm{M}+\mathrm{H}]^{+}: 373.1580$, found: 373.1578 .

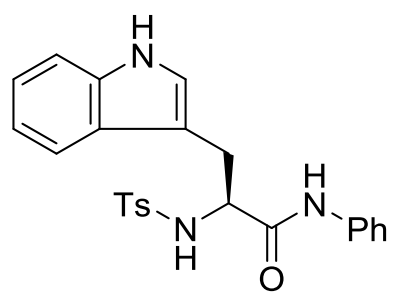

(S)-3-(1H-indol-3-yl)-2-((4-methylphenyl)sulfonamido)-N-phenylpropanamide (SI-2): Step 1: Prepared according a protocol Simsek and coworkers. ${ }^{25}$ To an oven-dried borosilicate test tube (L)-tryptophan ( $408 \mathrm{mg}, 2.0 \mathrm{mmol}$ ) is suspended/dissolved in $\mathrm{H}_{2} \mathrm{O} / \mathrm{THF}(9: 1$ ratio, $4.4 \mathrm{~mL}$ ) and set rapidly stirring. To this is added $\mathrm{NEt}_{3}(0.58 \mathrm{~mL}, 4.2 \mathrm{mmol})$ and the reaction tube is cooled to $0{ }^{\circ} \mathrm{C}$ in an ice water bath. After 15 minutes tosyl chloride (381 $\mathrm{mg}, 2.0 \mathrm{mmol}$ ) is added portion-wise over 15 minutes and the reaction is allowed to warm to ambient temperature. After consumption of starting material as determined by TLC the mixture was washed $2 \mathrm{x}$ with $\mathrm{Et}_{2} \mathrm{O}$, acidified with $1 \mathrm{M} \mathrm{HCl}$ and then extracted $3 \mathrm{x}$ with EtOAc. The combined EtOAc extracts were washed with brine, dried over $\mathrm{MgSO}_{4}$ and concentrated in vacuo. The crude ${ }^{1} \mathrm{H}$ NMR matched known spectra ${ }^{25}$ and was taken on without further purification.

Step 2: Prepared by charging a dry round bottomed flask with the crude tosyl protected product (717 $\mathrm{mg}$ ), DCC (413 mg, $2.0 \mathrm{mmol}, 1$ equiv.) 80\% 1-hydroxybenzenetriazole hydrate (34 mg, $0.2 \mathrm{mmol}, 0.1$ equiv.), purged $2 x$ with nitrogen and disolved in anh. TFH $(10 \mathrm{~mL})$. To this mixture aniline $(0.18 \mathrm{~mL}, 2.0$ mmol, 1 equiv.) was added dropwise over 15 minutes. The reaction mixture was allowed to stir at ambient temperature for $16 \mathrm{~h}$, the mixture was then concentrated in vacuo to give a residue which was

${ }^{25}$ S. Simsek, M. Horzella, M. Kalesse, M. Org. Lett. 2007, 9, 5637. 
purified by flash column chromatography (1:1 Hex: $\mathrm{CH}_{2} \mathrm{Cl}_{2}$ to $1: 9 \mathrm{MeOH}: \mathrm{CH}_{2} \mathrm{Cl}_{2}$ ) to give $\mathrm{SI}-2$ (633 mg, 1.4 $6 \mathrm{mmol}, 73 \%$ yield over two steps) as an off white solid.

Analytical data for SI-2: ${ }^{1} \mathrm{H}$ NMR $(500 \mathrm{MHz}$, Acetone) $\delta 9.96(\mathrm{~s}, 1 \mathrm{H}), 9.19(\mathrm{~s}, 1 \mathrm{H}), 7.61(\mathrm{~d}, J=8.3 \mathrm{~Hz}, 2 \mathrm{H})$, $7.51-7.47(\mathrm{~m}, 2 \mathrm{H}), 7.45(\mathrm{~d}, J=8.0 \mathrm{~Hz}, 1 \mathrm{H}), 7.35(\mathrm{~d}, J=8.1 \mathrm{~Hz}, 1 \mathrm{H}), 7.28-7.24(\mathrm{~m}, 2 \mathrm{H}), 7.15(\mathrm{~d}, J=2.1$ $\mathrm{Hz}, 1 \mathrm{H}), 7.10(\mathrm{dd}, J=11.6,4.5 \mathrm{~Hz}, 2 \mathrm{H}), 7.05(\mathrm{~d}, J=7.8 \mathrm{~Hz}, 2 \mathrm{H}), 6.98-6.94(\mathrm{~m}, 1 \mathrm{H}), 6.83(\mathrm{~d}, J=8.3 \mathrm{~Hz}$, $1 \mathrm{H}), 4.27$ (dd, $J=14.7,7.6 \mathrm{~Hz}, 1 \mathrm{H}$ ), 3.34 (dd, $J=14.5,6.5 \mathrm{~Hz}, 1 \mathrm{H}$ ), 3.15 (dd, $J=14.5,7.5 \mathrm{~Hz}, 1 \mathrm{H}$ ), 2.21 (s, $3 \mathrm{H}) ;{ }^{13} \mathrm{C}$ NMR (126 MHz, Acetone) $\delta$ 169.62, 143.00, 138.45, 137.41, 136.67, 129.26 (x2), 128.61 (x2), 127.46 (x2), 126.87, 124.05 (x2), 123.90, 121.31, 119.88 (x2), 118.78, 118.35, 111.41, 109.35, 58.29, 20.66; HRMS (ESI) calcd for $\left(\mathrm{C}_{24} \mathrm{H}_{24} \mathrm{~N}_{3} \mathrm{O}_{3} \mathrm{~S}\right)^{+}[\mathrm{M}+\mathrm{H}]^{+}: 434.1533$, found: 434.1515 .

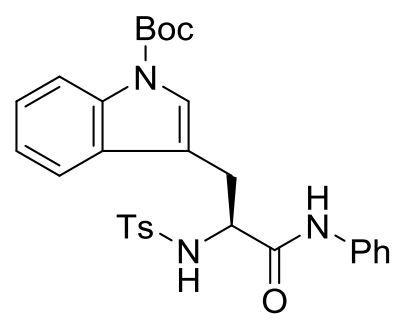

tert-butyl (S)-3-(2-((4-methylphenyl)sulfonamido)-3-oxo-3-(phenylamino)propyl)-1H-indole-1carboxylate (5I): Step 3: Selective protection of the indole nitrogen was accomplished by following a modified procedure from Chalasani and coworkers. ${ }^{3}$ To an oven dried borosilicate test tube charged with SI-2 (127 mg, $0.29 \mathrm{mmol}$ ) is added DMAP (4-dimethylamino pyridine) ( $4 \mathrm{mg}, 0.03 \mathrm{mmol}$ ) and sealed under $\mathrm{N}_{2}$. The solids were then dissolved in anh. $\mathrm{CH}_{2} \mathrm{Cl}_{2}(3.0 \mathrm{~mL})$ followed by addition of $\mathrm{N}(i \operatorname{Pr})_{2} \mathrm{Et}(0.13$ $\mathrm{mL}, 0.73 \mathrm{mmol}$ ) and then cooled to $0{ }^{\circ} \mathrm{C}$ in an ice water bath. $\mathrm{Boc}_{2} \mathrm{O}(63 \mathrm{mg}, 0.29 \mathrm{mmol}$ ) was dissolved in anh. $\mathrm{CH}_{2} \mathrm{Cl}_{2}(1.0 \mathrm{~mL})$ and added dropwise over 90 minutes via syringe to the solution. Following addition the reaction mixture was allowed to warm to ambient temperature and stirred an additional $12 \mathrm{~h}$. The reaction mixture was then diluted with $\mathrm{CH}_{2} \mathrm{Cl}_{2}$ and subsequently washed with $0.5 \mathrm{M} \mathrm{HCl}_{(\mathrm{aq})}$, water, brine, dried over $\mathrm{Na}_{2} \mathrm{SO}_{4}$ and concentrated in vacuo. The resulting residue was purified by flash column chromatography (9:1-5:2 Hex:EtOAc) to give $5 \mathrm{I}$ (33 $\mathrm{mg}, 0.06 \mathrm{mmol}, 22 \%$ yield, $78 \% \mathrm{BRSM}$ ) as a white amorphous solid.

Analytical data for $5 \mathrm{I}:{ }^{1} \mathrm{H}$ NMR $\left(500 \mathrm{MHz}, \mathrm{CDCl}_{3}\right) \delta 8.38(\mathrm{~s}, 1 \mathrm{H}), 8.04(\mathrm{~s}, 1 \mathrm{H}), 7.44(\mathrm{~d}, J=7.7 \mathrm{~Hz}, 2 \mathrm{H}), 7.39$ $(\mathrm{d}, J=8.2 \mathrm{~Hz}, 2 \mathrm{H}), 7.34-7.25(\mathrm{~m}, 5 \mathrm{H}), 7.11(\mathrm{dd}, J=13.8,6.9 \mathrm{~Hz}, 2 \mathrm{H}), 6.90(\mathrm{~d}, J=8.1 \mathrm{~Hz}, 2 \mathrm{H}), 5.24(\mathrm{~d}, J=$ $6.2 \mathrm{~Hz}, 1 \mathrm{H}), 4.03(\mathrm{dt}, J=8.7,5.6 \mathrm{~Hz}, 1 \mathrm{H}), 3.30(\mathrm{dd}, J=14.8,4.9 \mathrm{~Hz}, 1 \mathrm{H}), 2.97(\mathrm{dd}, J=14.8,8.8 \mathrm{~Hz}, 1 \mathrm{H})$, $2.27(\mathrm{~s}, 3 \mathrm{H}), 1.65(\mathrm{~s}, 9 \mathrm{H}) ;{ }^{13} \mathrm{C} \mathrm{NMR}\left(126 \mathrm{MHz}, \mathrm{CDCl}_{3}\right) \delta 168.45,143.99,137.04,129.35(\mathrm{x} 2), 128.92(\mathrm{x} 2)$, 126.71 (x2), 124.79 (x2), 124.62 (x2), 124.50, 122.78 (x2), $120.20(x 2), 118.61(x 2), 115.32(x 2), 114.32$, 83.87, 57.06, $28.16(x 3), 21.56$; HRMS (ESI) calcd for $\left(\mathrm{C}_{29} \mathrm{H}_{32} \mathrm{~N}_{3} \mathrm{O}_{5} \mathrm{~S}\right)^{+}[\mathrm{M}+\mathrm{H}]^{+}: 534.3057$, found: 534.3059. 


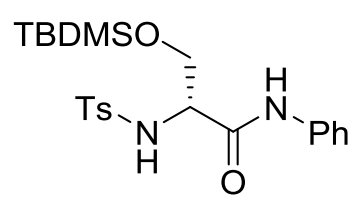

(R)-3-((tert-butyldimethylsilyl)oxy)-2-((4-methylphenyl)sulfonamido)- $\mathrm{N}$-phenylpropanamide $\quad$ (5m): Step 1: Prepared according a modified protocol from Craig and coworkers. ${ }^{26}$ To a round bottomed flask charged with $(D)$-serine $(210 \mathrm{mg}, 2.0 \mathrm{mmol}$ ) was added tosyl chloride (496 mg, $2.6 \mathrm{mmol}$ ) EtOAc (4.8 mL) and water $(1.4 \mathrm{~mL})$. The suspension was set rapidly stirring and $2.0 \mathrm{M} \mathrm{NaOH}_{\text {(aq) }}(2.7 \mathrm{~mL}, 5.4 \mathrm{mmol})$ was added dropwise over $3 \mathrm{~h}$ at ambient temperature. After addition the reaction mixture was allowed to stir for an additional hour. At this point the layers were separated and the aqueous layer was acidified with $\mathrm{HCl}_{\text {(conc) }}$ and precipitate was isolated by vacuum filtration. The precipitate was washed with water and dried to give the crude tosyl protected intermediate.

Step 2: The crude product $(0.27 \mathrm{~g}, 1.0 \mathrm{mmol})$ was dissolved in anh. THF $(20 \mathrm{~mL})$ and cooled to $0{ }^{\circ} \mathrm{C}$ in an ice water bath. To this mixture imidazole $(170 \mathrm{mg}, 2.5 \mathrm{mmol})$ was added and the reaction mixture was sealed under $\mathrm{N}_{2}$. TBDMSCl (166 mg, $1.1 \mathrm{mmol}$ ) was added portion-wise over $20 \mathrm{~min}$, after completion of addition the reaction was purged with $\mathrm{N}_{2}$, allowed to warm to ambient temperature and stir an additional $12 \mathrm{~h}$. The mixture was then diluted with EtOAc, washed with $1 \mathrm{M} \mathrm{HCl}$, water and brine, and concentrated in vacuo. The resulting residue was taken on without further purification.

Step 3: Prepared according to the general method using crude residue from the previous step (234 $\mathrm{mg}$ ), $\mathrm{SOCl}_{2}(0.11 \mathrm{~mL}, 1.46 \mathrm{mmol})$, aniline $(0.06 \mathrm{~mL}, 0.69 \mathrm{mmol})$ and THF $(6.3 \mathrm{~mL})$ were stirred for $16 \mathrm{~h}$ at ambient temperature. Purified by flash column chromatography $\left(4: 1\right.$ to $3: 1$ Hexanes: $\left.\mathrm{CH}_{2} \mathrm{Cl}_{2}\right)$ to afford $5 \mathrm{~m}$ (100 mg, $0.32 \mathrm{mmol}, 51 \%$ yield) as an off white amorphous solid.

Analytical data for $5 \mathrm{~m}:{ }^{1} \mathrm{H}$ NMR $\left(500 \mathrm{MHz}, \mathrm{CDCl}_{3}\right) \delta 8.60(\mathrm{~s}, 1 \mathrm{H}), 7.78(\mathrm{~d}, J=8.3 \mathrm{~Hz}, 2 \mathrm{H}), 7.43(\mathrm{~d}, J=7.7$ $\mathrm{Hz}, 2 \mathrm{H}), 7.35-7.28(\mathrm{~m}, 3 \mathrm{H}), 7.12(\mathrm{t}, J=7.4 \mathrm{~Hz}, 1 \mathrm{H}), 5.72(\mathrm{~d}, J=5.4 \mathrm{~Hz}, 1 \mathrm{H}), 4.12(\mathrm{dd}, J=10.1,3.8 \mathrm{~Hz}, 1 \mathrm{H})$, $3.72(\mathrm{ddd}, J=7.2,5.5,3.9 \mathrm{~Hz}, 1 \mathrm{H}), 3.54(\mathrm{dd}, J=10.1,7.3 \mathrm{~Hz}, 1 \mathrm{H}), 2.42(\mathrm{~s}, 3 \mathrm{H}), 0.87(\mathrm{~s}, 9 \mathrm{H}), 0.06$ (d, $J=4.6$ $\mathrm{Hz}, 6 \mathrm{H}) ;{ }^{13} \mathrm{C}$ NMR (126 MHz, $\left.\mathrm{CDCl}_{3}\right) \delta 167.11,144.26,137.10,130.00$ (x2), 129.02 (x2), 127.32 (x2), 124.73 (x2), 119.89 (x2), 63.21, 57.51, 25.76 (x3), 21.52, 18.13, -5.51, -5.56; HRMS (ESI) calcd for $\left(\mathrm{C}_{22} \mathrm{H}_{32} \mathrm{~N}_{2} \mathrm{O}_{4} \mathrm{SSiNa}\right)^{+}[\mathrm{M}+\mathrm{Na}]^{+}:$471.1744, found: 471.1736 .

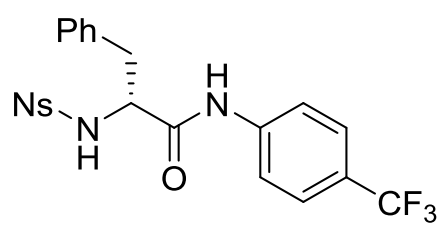

\section{(R)-2-((4-nitrophenyl)sulfonamido)-3-phenyl-N-(4-(trifluoromethyl)phenyl)propanamide}

(5n): Prepared according to the general method using $(D)$-phenylalanine (1.65 g, $10.0 \mathrm{mmol}), 2$-nitrosulfonyl chloride $(2.66 \mathrm{~g}, 12.0 \mathrm{mmol}), \mathrm{K}_{2} \mathrm{CO}_{3}(2.90 \mathrm{~g}, 21 \mathrm{mmol})$ and water $(12.0 \mathrm{~mL})$ for $16 \mathrm{~h}$. A portion of the crude material was taken on to the next step. The crude solid $(0.60 \mathrm{~g}), \mathrm{SOCl}_{2}(0.29 \mathrm{~mL}, 3.98 \mathrm{mmol})$, 4-

${ }^{26}$ D. Craig, C. J. T. Hyland, S. E. Ward, S. E. Chem. Commun. 2005, 3439. 
(trifluoromethyl)aniline $(0.24 \mathrm{~mL}, 1.90 \mathrm{mmol})$ and THF $(65 \mathrm{~mL})$ were stirred for $16 \mathrm{~h}$ at ambient temperature. Purified by flash column chromatography (9:1 to 5:2 Hexanes:EtOAc) to afford $\mathbf{5 n}$ ( $244 \mathrm{mg}$, $0.49 \mathrm{mmol}, 29 \%$ yield over two steps) as a yellow amorphous solid.

Analytical data for $5 \mathrm{n}:{ }^{1} \mathrm{H}$ NMR $\left(500 \mathrm{MHz}, \mathrm{CDCl}_{3}\right) \delta 8.76(\mathrm{~s}, 1 \mathrm{H}), 8.10-7.91(\mathrm{~m}, 1 \mathrm{H}), 7.77-7.70(\mathrm{~m}, 1 \mathrm{H})$, $7.67-7.56(\mathrm{~m}, 4 \mathrm{H}), 7.47(\mathrm{~d}, J=8.6 \mathrm{~Hz}, 2 \mathrm{H}), 7.08-7.02(\mathrm{~m}, 2 \mathrm{H}), 6.98(\mathrm{t}, J=6.9 \mathrm{~Hz}, 3 \mathrm{H}), 6.49(\mathrm{~d}, J=5.4 \mathrm{~Hz}$, $1 \mathrm{H}), 4.48-4.22(\mathrm{~m}, 1 \mathrm{H}), 3.38(\mathrm{dd}, J=14.2,4.7 \mathrm{~Hz}, 1 \mathrm{H}), 2.99(\mathrm{dd}, J=14.2,10.3 \mathrm{~Hz}, 1 \mathrm{H}) ;{ }^{13} \mathrm{C}$ NMR $(126$ $\left.\mathrm{MHz}_{1} \mathrm{CDCl}_{3}\right) \delta 169.04,146.85,140.04,134.88,133.86,133.25,132.18,131.02,128.93$ (x2), 128.54, $127.21,126.03,126.00,125.97,125.88,125.00,122.84,119.87$ (x2), 60.71, 38.19; HRMS (ESI) calcd for $\left(\mathrm{C}_{22} \mathrm{H}_{19} \mathrm{~F}_{3} \mathrm{~N}_{3} \mathrm{O}_{5} \mathrm{~S}\right)^{+}[\mathrm{M}+\mathrm{H}]^{+}: 494.0992$, found: 494.0991 .

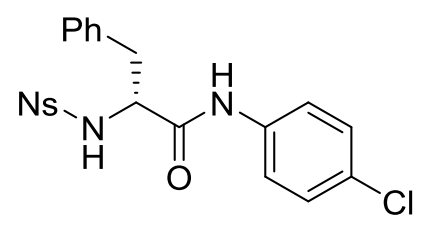

(R)-N-(4-chlorophenyl)-2-((4-nitrophenyl)sulfonamido)-3-phenylpropanamide (5o): Prepared according to the general method using $(D)$-phenylalanine $(1.65 \mathrm{~g}, 10.0 \mathrm{mmol})$, 2-nitrosulfonyl chloride (2.66 g, 12.0 $\mathrm{mmol}), \mathrm{K}_{2} \mathrm{CO}_{3}(2.90 \mathrm{~g}, 21 \mathrm{mmol})$ and water $(12.0 \mathrm{~mL})$ for $16 \mathrm{~h}$. A portion of the crude material was taken on to the next step. The crude solid $(2.74 \mathrm{~g}), \mathrm{SOCl}_{2}(1.33 \mathrm{~mL}, 18.2 \mathrm{mmol})$, 4-chloroaniline $(1.10 \mathrm{~g}, 8.6$ $\mathrm{mmol}$ ) and THF $(200 \mathrm{~mL})$ were stirred for $16 \mathrm{~h}$ at ambient temperature. Purified by flash column chromatography ( $9: 1$ to 5:2 Hexanes:EtOAc) to afford 50 ( $1.24 \mathrm{~g}, 2.69 \mathrm{mmol}, 35 \%$ yield over two steps) as a yellow amorphous solid.

Analytical data for $50:{ }^{1} \mathrm{H}$ NMR $\left(500 \mathrm{MHz}, \mathrm{CDCl}_{3}\right) \delta 8.50(\mathrm{~s}, 1 \mathrm{H}), 8.03-7.92(\mathrm{~m}, 1 \mathrm{H}), 7.73$ (dd, J = 7.0, 1.1 $\mathrm{Hz}, 1 \mathrm{H}), 7.69-7.58(\mathrm{~m}, 2 \mathrm{H}), 7.38(\mathrm{~d}, J=8.6 \mathrm{~Hz}, 2 \mathrm{H}), 7.20(\mathrm{~d}, J=8.6 \mathrm{~Hz}, 2 \mathrm{H}), 7.01(\mathrm{~d}, J=8.4 \mathrm{~Hz}, 5 \mathrm{H}), 6.39$ $(\mathrm{d}, J=5.7 \mathrm{~Hz}, 1 \mathrm{H}), 4.34-4.18(\mathrm{~m}, 1 \mathrm{H}), 3.33(\mathrm{dd}, J=14.2,4.8 \mathrm{~Hz}, 1 \mathrm{H}), 2.96(\mathrm{dd}, J=14.1,9.9 \mathrm{~Hz}, 1 \mathrm{H}) ;{ }^{13} \mathrm{C}$ NMR $\left(126 \mathrm{MHz}, \mathrm{CDCl}_{3}\right) \delta 168.52,146.94,135.47,134.95,133.80,133.17,132.34,130.99,129.86$, $128.95,128.85,128.58,127.21,125.86,121.54$ (x2), 60.48, 38.32, 31.49, 22.56, 14.02; HRMS (ESI) calcd for $\left(\mathrm{C}_{21} \mathrm{H}_{19} \mathrm{ClN}_{3} \mathrm{O}_{5} \mathrm{~S}\right)^{+}[\mathrm{M}+\mathrm{H}]^{+}: 460.0728$, found: 460.0726 .

\section{General Procedure for the Pd-catalyzed Piperizine Formation}

General Method: Diamine substrate (1 equiv.), $\mathrm{Pd}_{2}(\mathrm{dba})_{3} \cdot \mathrm{CHCl}_{3}(0.015$ equiv., $3 \mathrm{~mol} \% \mathrm{Pd}$ ) and DPEphos ( 0.033 equiv.) were added to a oven-dried $16 \times 100$ borosilicate test tube. The test tube was covered with a rubber septum and sealed with Teflon tape. The test tube was purged with nitrogen, anhydrous $\mathrm{CH}_{2} \mathrm{Cl}_{2}$ $(0.1 \mathrm{M})$ was added and the mixture was maintained under a positive pressure of nitrogen. In the case of a heterogeneous mixture sonication was used to break up larger particles of insoluble substrate. After 2 min tert-butyl propargyl carbonate $\mathbf{2 a}$ (1.3 equiv.) was added to the reaction mixture via syringe. The test tube was purged again with nitrogen. After consumption of starting material as judged by TLC, the reaction was concentrated and purified by flash column chromatography to give the desired products. 
<smiles>C=C1CN([13CH3])CCN1[13OH]</smiles>

2-methylene-1,4-ditosylpiperazine (3a): Prepared according to the general method using 1a (184 mg, $0.5 \mathrm{mmol}$ ), $\mathrm{Pd}_{2}(\mathrm{dba})_{3} \cdot \mathrm{CHCl}_{3}(2.6 \mathrm{mg}, 2.5 \mu \mathrm{mol})$, DPEphos (3.2 mg, $5.5 \mu \mathrm{mol}$ ), propargyl carbonate $2 \mathrm{a}$ (102 $\mathrm{mg}, 0.65 \mathrm{mmol}$ ) in $\mathrm{CH}_{2} \mathrm{Cl}_{2}(5.0 \mathrm{~mL})$ for $12 \mathrm{~h}$. Purified by flash column chromatography (9:1 to $3: 1$ Hexanes:EtOAc) to afford $3 a$ ( $165 \mathrm{mg}, 0.43 \mathrm{mmol}, 85 \%$ yield) as a white solid.

Analytical data for $3 a:\left(\mathrm{mp}: 169{ }^{\circ} \mathrm{C}\right),{ }^{1} \mathrm{H}$ NMR $\left(500 \mathrm{MHz}, \mathrm{CDCl}_{3}\right) \delta 7.61(\mathrm{~d}, J=8.3 \mathrm{~Hz}, 2 \mathrm{H}), 7.56(\mathrm{~d}, J=8.3$ $\mathrm{Hz}, 2 \mathrm{H}), 7.31(\mathrm{~d}, J=7.9 \mathrm{~Hz}, 2 \mathrm{H}), 7.22(\mathrm{~d}, J=7.9 \mathrm{~Hz}, 2 \mathrm{H}), 5.11(\mathrm{~d}, J=0.6 \mathrm{~Hz}, 1 \mathrm{H}), 4.96(\mathrm{~d}, J=0.7 \mathrm{~Hz}, 1 \mathrm{H})$, $3.79-3.63(\mathrm{~m}, 2 \mathrm{H}), 3.36(\mathrm{~s}, 2 \mathrm{H}), 3.09-2.99(\mathrm{~m}, 2 \mathrm{H}), 2.45(\mathrm{~s}, 3 \mathrm{H}), 2.39(\mathrm{~s}, 3 \mathrm{H}) ;{ }^{13} \mathrm{C} \mathrm{NMR}\left(126 \mathrm{MHz}, \mathrm{CDCl}_{3}\right)$ $\delta$ 144.09, 143.97, 136.73 (x2), 136.05 (x2), 129.75, 129.69 (x2), 127.73 (x2), 127.15 (x2), 110.30, 49.61, 45.87, 44.68, 21.52, 21.46; HRMS (ESI) calcd for $\left(\mathrm{C}_{19} \mathrm{H}_{23} \mathrm{~N}_{2} \mathrm{O}_{4} \mathrm{~S}_{2}\right)^{+}[\mathrm{M}+\mathrm{H}]^{+}:$407.1094, found: 407.1099.<smiles>C=C1CN(S(=O)(=O)Oc2ccccc2)CCN1S(=O)(=O)c1ccccc1</smiles>

2-methylene-1,4-bis(phenylsulfonyl)piperazine (3b): Prepared according to the general method using 1b $(68 \mathrm{mg}, 0.2 \mathrm{mmol}), \mathrm{Pd}_{2}(\mathrm{dba})_{3} \cdot \mathrm{CHCl}_{3}(3.1 \mathrm{mg}, 3.0 \mu \mathrm{mol})$, DPEphos (3.6 mg, $\left.6.6 \mu \mathrm{mol}\right)$, propargyl carbonate $2 \mathrm{a}(41 \mathrm{mg}, 0.26 \mathrm{mmol})$ in $\mathrm{CH}_{2} \mathrm{Cl}_{2}(2.0 \mathrm{~mL})$ for $20 \mathrm{~min}$. Purified by flash column chromatography (9:1 to $3: 1$ Hexanes:EtOAc) to afford $\mathbf{3 b}$ ( $70 \mathrm{mg}, 0.19 \mathrm{mmol}, 93 \%$ yield) as a white solid.

Analytical data for $\mathbf{3 b}$ : (mp: $\left.138^{\circ} \mathrm{C}\right),{ }^{1} \mathrm{H}$ NMR $\left(500 \mathrm{MHz}, \mathrm{CDCl}_{3}\right) \delta 7.72(\mathrm{dd}, J=8.3,1.1 \mathrm{~Hz}, 2 \mathrm{H}), 7.66$ (dd, $J$ $=8.2,1.0 \mathrm{~Hz}, 2 \mathrm{H}), 7.63(\mathrm{t}, J=7.5 \mathrm{~Hz}, 1 \mathrm{H}), 7.57-7.46(\mathrm{~m}, 3 \mathrm{H}), 7.42(\mathrm{t}, J=7.8 \mathrm{~Hz}, 2 \mathrm{H}), 5.16(\mathrm{~s}, 1 \mathrm{H}), 5.00(\mathrm{~s}$, $1 \mathrm{H}), 3.76-3.66(\mathrm{~m}, 2 \mathrm{H}), 3.35(\mathrm{~s}, 2 \mathrm{H}), 3.11-2.94(\mathrm{~m}, 2 \mathrm{H}) ;{ }^{13} \mathrm{C} \mathrm{NMR}\left(126 \mathrm{MHz}, \mathrm{CDCl}_{3}\right) \delta$ 135.89, 133.21 (x2), 133.06 (x2), 129.18 (x2), 129.13 (x2), 127.66 (x2), 127.04 (x2), 110.92, 49.48, 45.95, 44.58; HRMS (ESI) calcd for $\left(\mathrm{C}_{17} \mathrm{H}_{19} \mathrm{~N}_{2} \mathrm{O}_{4} \mathrm{~S}_{2}\right)^{+}[\mathrm{M}+\mathrm{H}]^{+}$: 379.0781, found: 379.0792 .

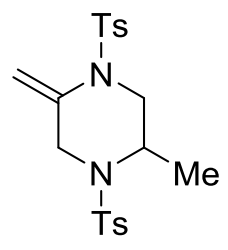

2-methyl-5-methylene-1,4-ditosylpiperazine (3c): Prepared according to the general method using 1c (77 mg, $0.2 \mathrm{mmol}), \mathrm{Pd}_{2}(\mathrm{dba})_{3} \cdot \mathrm{CHCl}_{3}(3.1 \mathrm{mg}, 3.0 \mu \mathrm{mol})$, DPEphos (3.6 mg, $6.6 \mu \mathrm{mol}$ ), propargyl carbonate 2a (41 mg, $0.26 \mathrm{mmol})$ in $\mathrm{CH}_{2} \mathrm{Cl}_{2}(2.0 \mathrm{~mL})$ for $3 \mathrm{~h}$ at $0{ }^{\circ} \mathrm{C}$. Purified by flash column chromatography (9:1 to $3: 1$ Hexanes:EtOAc) to afford $3 c$ ( $82 \mathrm{mg}, 0.19 \mathrm{mmol}, 97 \%$ yield) as a white solid.

Analytical data for $3 \mathrm{c}:\left(\mathrm{mp}: 145^{\circ} \mathrm{C}\right),{ }^{1} \mathrm{H}$ NMR $\left(500 \mathrm{MHz}, \mathrm{CDCl}_{3}\right) \delta 7.61(\mathrm{dt}, J=16.5,8.2 \mathrm{~Hz}, 4 \mathrm{H}), 7.51(\mathrm{~d}, J=$ $8.2 \mathrm{~Hz}, \mathrm{OH}), 7.34-7.23(\mathrm{~m}, 4 \mathrm{H}), 7.17(\mathrm{~d}, J=8.0 \mathrm{~Hz}, \mathrm{OH}), 5.24(\mathrm{~s}, \mathrm{OH}), 5.13(\mathrm{~s}, \mathrm{OH}), 5.02(\mathrm{~s}, 1 \mathrm{H}), 4.66(\mathrm{~s}$, $1 \mathrm{H}), 4.11-3.99(\mathrm{~m}, 1 \mathrm{H}), 3.91(\mathrm{~d}, J=12.1 \mathrm{~Hz}, \mathrm{OH}), 3.78(\mathrm{~d}, J=14.2 \mathrm{~Hz}, 1 \mathrm{H}), 3.72(\mathrm{~d}, J=14.2 \mathrm{~Hz}, 1 \mathrm{H}), 3.56$ 
$(\mathrm{dd}, J=12.8,4.6 \mathrm{~Hz}, 1 \mathrm{H}), 3.34(\mathrm{dd}, J=12.8,4.0 \mathrm{~Hz}, 1 \mathrm{H}), 2.43(\mathrm{~d}, J=12.6 \mathrm{~Hz}, 6 \mathrm{H}), 2.36(\mathrm{~s}, 0 \mathrm{H}), 1.30(\mathrm{~d}, J=$ $6.8 \mathrm{~Hz}, \mathrm{OH}), 1.23(\mathrm{~d}, J=6.6 \mathrm{~Hz}, 3 \mathrm{H}) ;{ }^{13} \mathrm{C} \mathrm{NMR}\left(126 \mathrm{MHz} \mathrm{CDCl}_{3}\right) \delta 144.09,144.06,143.68,136.61,135.84$, 129.70 (x2), 129.64, 129.52 (x2), 127.63 (x2), 127.29 (x2), 127.23, 127.18, 114.46, 102.89, 50.42, 50.09, 49.85, 49.32, 49.21, 46.41, 21.48, 21.46, 17.93; HRMS (ESI) calcd for $\left(\mathrm{C}_{20} \mathrm{H}_{25} \mathrm{~N}_{2} \mathrm{O}_{4} \mathrm{~S}_{2}\right)^{+}[\mathrm{M}+\mathrm{H}]^{+}: 421.1250$, found: 421.1265 .

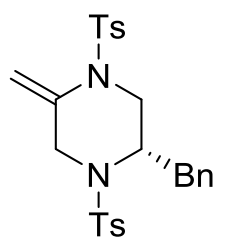

(S)-2-benzyl-5-methylene-1,4-ditosylpiperazine (3d): Prepared according to the general method using 1d (92 mg, $0.2 \mathrm{mmol}), \mathrm{Pd}_{2}(\mathrm{dba})_{3} \cdot \mathrm{CHCl}_{3}(3.1 \mathrm{mg}, 3.0 \mu \mathrm{mol})$, DPEphos $(3.6 \mathrm{mg}, 6.6 \mu \mathrm{mol})$, propargyl carbonate $2 \mathrm{a}$ ( $41 \mathrm{mg}, 0.26 \mathrm{mmol})$ in $\mathrm{CH}_{2} \mathrm{Cl}_{2}(2.0 \mathrm{~mL})$ for $15 \mathrm{~min}$. Purified by flash column chromatography (9:1 to 3:1 Hexanes:EtOAc) to afford $\mathbf{3 d}(79 \mathrm{mg}, 0.16 \mathrm{mmol}, 80 \%$ yield) as a white solid.

Analytical data for $\mathbf{3 d}$ : $\left(\mathrm{mp}: 64{ }^{\circ} \mathrm{C}\right),{ }^{1} \mathrm{H}$ NMR $\left(500 \mathrm{MHz}, \mathrm{CDCl}_{3}\right) \delta 7.59(\mathrm{~d}, \mathrm{~J}=8.1 \mathrm{~Hz}, 2 \mathrm{H}), 7.56(\mathrm{~d}, \mathrm{~J}=8.2 \mathrm{~Hz}$, $2 \mathrm{H}), 7.31(\mathrm{t}, \mathrm{J}=7.3 \mathrm{~Hz}, 2 \mathrm{H}), 7.27-7.22(\mathrm{~m}, 7 \mathrm{H}), 5.11(\mathrm{~s}, 1 \mathrm{H}), 4.72(\mathrm{~s}, 1 \mathrm{H}), 4.06(\mathrm{dd}, \mathrm{J}=9.3,4.3 \mathrm{~Hz}, 1 \mathrm{H})$, $3.76-3.67(\mathrm{~m}, 2 \mathrm{H}), 3.67-3.62(\mathrm{~m}, 1 \mathrm{H}), 3.08-2.94(\mathrm{~m}, 3 \mathrm{H}), 2.42(\mathrm{~d}, \mathrm{~J}=7.0 \mathrm{~Hz}, 6 \mathrm{H}) ;{ }^{13} \mathrm{C} \mathrm{NMR}(126 \mathrm{MHz}$, $\left.\mathrm{CDCl}_{3}\right) \delta 144.12,143.78,136.90,136.74,129.75$ (x2), 129.67 (x2), 129.57 (x2), 128.70 (x2), 127.39 (x3), 127.26 (x2), 126.87 (x2), 103.38, 56.00, 47.25, 46.50, 38.47, 21.54, 21.51; HRMS (ESI) calcd for $\left(\mathrm{C}_{26} \mathrm{H}_{29} \mathrm{~N}_{2} \mathrm{O}_{4} \mathrm{~S}_{2}\right)^{+}[\mathrm{M}+\mathrm{H}]^{+}:$497.1563, found: 497.1564 .

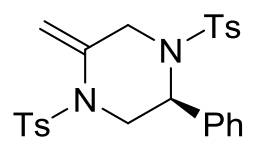

(S)-2-phenyl-5-methylene-1,4-ditosylpiperazine (3e): Prepared according to the general method using 1e (133 mg, $0.3 \mathrm{mmol}), \mathrm{Pd}_{2}\left(\mathrm{dba}_{3} \cdot \mathrm{CHCl}_{3}(4.6 \mathrm{mg}, 4.5 \mu \mathrm{mol})\right.$, DPEphos (5.3 mg, $\left.9.9 \mu \mathrm{mol}\right)$, propargyl carbonate $2 \mathrm{a}(61 \mathrm{mg}, 0.39 \mathrm{mmol})$ in $\mathrm{CH}_{2} \mathrm{Cl}_{2}(3.0 \mathrm{~mL})$ for $10 \mathrm{~min}$. Purified by flash column chromatography (6:1 to $3: 1$ Hexanes: $\mathrm{CH}_{2} \mathrm{Cl}_{2}$ ) to afford $3 e(134 \mathrm{mg}, 0.28 \mathrm{mmol}, 92 \%$ yield) as an off white amorphous solid.

Analytical data for $3 \mathrm{e}:\left(\mathrm{mp}: 11{ }^{\circ} \mathrm{C}\right),{ }^{1} \mathrm{H}$ NMR $\left(500 \mathrm{MHz}, \mathrm{CDCl}_{3}\right) \delta 7.58(\mathrm{~d}, J=8.3 \mathrm{~Hz}, 2 \mathrm{H}), 7.44(\mathrm{~d}, J=8.3$ $\mathrm{Hz}, 2 \mathrm{H}), 7.36-7.28(\mathrm{~m}, 5 \mathrm{H}), 7.22(\mathrm{~d}, J=7.9 \mathrm{~Hz}, 2 \mathrm{H}), 7.18(\mathrm{~d}, J=8.0 \mathrm{~Hz}, 2 \mathrm{H}), 5.01-4.97(\mathrm{~m}, 1 \mathrm{H}), 4.95(\mathrm{~s}$, $1 \mathrm{H}), 4.52(\mathrm{~s}, 1 \mathrm{H}), 4.08(\mathrm{~d}, J=15.1 \mathrm{~Hz}, 1 \mathrm{H}), 3.88(\mathrm{~d}, J=15.0 \mathrm{~Hz}, 1 \mathrm{H}), 3.84(\mathrm{dd}, J=13.5,6.8 \mathrm{~Hz}, 1 \mathrm{H}), 3.75$ $(\mathrm{dd}, J=13.5,4.8 \mathrm{~Hz}, 1 \mathrm{H}), 2.41(\mathrm{~s}, 3 \mathrm{H}), 2.39(\mathrm{~s}, 3 \mathrm{H}) ;{ }^{13} \mathrm{C} \mathrm{NMR}\left(126 \mathrm{MHz}, \mathrm{CDCl}_{3}\right) \delta 144.09,143.76,138.15$, 136.64, 135.73, 135.19, 129.62, 129.59, 128.65, 127.93, 127.45, 127.25, 126.57, 100.21, 57.94, 48.69, 47.83, 21.48; HRMS (ESI) calcd for $\left(\mathrm{C}_{25} \mathrm{H}_{27} \mathrm{~N}_{2} \mathrm{O}_{4} \mathrm{~S}_{2}\right)^{+}[\mathrm{M}+\mathrm{H}]^{+}: 483.1407$, found: 483.1410 . 


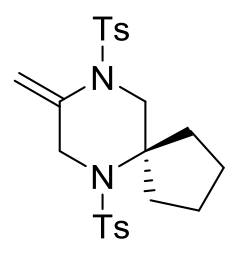

8-methylene-6,9-ditosyl-6,9-diazaspiro[4.5]decane (3f): Prepared according to the general method using $1 \mathrm{f}\left(106 \mathrm{mg}, 0.25 \mathrm{mmol}\right.$ ), $\mathrm{Pd}_{2}\left(\mathrm{dba}_{3} \cdot \mathrm{CHCl}_{3}\right.$ (3.9 mg, $3.8 \mu \mathrm{mol}$ ), DPEphos (4.5 mg, $\left.8.3 \mu \mathrm{mol}\right)$, propargyl carbonate $2 \mathrm{a}$ ( $52 \mathrm{mg}, 0.33 \mathrm{mmol}$ ) in $\mathrm{CH}_{2} \mathrm{Cl}_{2}(2.5 \mathrm{~mL}$ ) for $45 \mathrm{~min}$. Purified by flash column chromatography (9:1 to 4:1 Hexanes:EtOAc) to afford $\mathbf{3 f}$ (113 $\mathrm{mg}, 0.25 \mathrm{mmol}, 98 \%$ yield) as a white amorphous solid.

Analytical data for $3 f:\left(\mathrm{mp}: 138{ }^{\circ} \mathrm{C}\right),{ }^{1} \mathrm{H}$ NMR $\left(500 \mathrm{MHz}, \mathrm{CDCl}_{3}\right) \delta 7.68(\mathrm{~d}, \mathrm{~J}=8.2 \mathrm{~Hz}, 2 \mathrm{H}), 7.65(\mathrm{~d}, \mathrm{~J}=8.2$ $\mathrm{Hz}, 2 \mathrm{H}), 7.30(\mathrm{~d}, \mathrm{~J}=8.0 \mathrm{~Hz}, 2 \mathrm{H}), 7.24(\mathrm{~d}, \mathrm{~J}=8.0 \mathrm{~Hz}, 2 \mathrm{H}), 5.02(\mathrm{~s}, 1 \mathrm{H}), 4.58(\mathrm{~s}, 1 \mathrm{H}), 3.95(\mathrm{~s}, 2 \mathrm{H}), 3.47(\mathrm{~s}, 2 \mathrm{H})$, $2.43(\mathrm{~s}, 3 \mathrm{H}), 2.40(\mathrm{~s}, 3 \mathrm{H}), 2.25-2.17(\mathrm{~m}, 2 \mathrm{H}), 1.80(\mathrm{t}, \mathrm{J}=8.8 \mathrm{~Hz}, 4 \mathrm{H}), 1.60-1.52(\mathrm{~m}, 2 \mathrm{H}) ;{ }^{13} \mathrm{C}$ NMR $(126$ $\mathrm{MHz}, \mathrm{CDCl}_{3}$ ) $\delta 143.19,143.17,137.72,129.72$ (x2), 129.59 (x2), 127.19 (x3), 126.90 (x3), 100.72, 69.32, 53.04, 49.46, $34.94(\times 2), 23.18(\times 2), 21.52,21.43$; HRMS (ESI) calcd for $\left(\mathrm{C}_{23} \mathrm{H}_{28} \mathrm{~N}_{2} \mathrm{O}_{4} \mathrm{~S}_{2} \mathrm{Na}\right)^{+}[\mathrm{M}+\mathrm{Na}]^{+}$: 483.1388, found: 483.1384 .

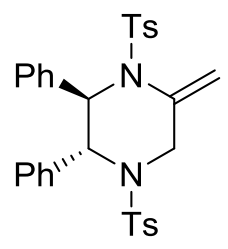

(2R,3R)-5-methylene-2,3-diphenyl-1,4-ditosylpiperazine (3g): Prepared according to the general

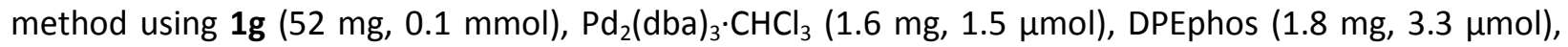
propargyl carbonate $2 \mathrm{a}(20 \mathrm{mg}, 0.13 \mathrm{mmol})$ in $\mathrm{CH}_{2} \mathrm{Cl}_{2}(1.0 \mathrm{~mL})$ for $60 \mathrm{~min}$. Purified by flash column chromatography (6:1 to $3: 1$ Hexanes:EtOAc) to afford $3 \mathrm{~g}(52 \mathrm{mg}, 0.09 \mathrm{mmol}, 94 \%$ yield) as a white amorphous solid.

Analytical data for $3 \mathrm{~g}$ : (mp: $\left.247^{\circ} \mathrm{C}\right),{ }^{1} \mathrm{H}$ NMR $\left(500 \mathrm{MHz}, \mathrm{CDCl}_{3}\right) \delta 7.57-7.46(\mathrm{~m}, 2 \mathrm{H}), 7.37$ (ddd, $J=6.7$, 4.3, $1.5 \mathrm{~Hz}, 5 \mathrm{H}), 7.25-7.20(\mathrm{~m}, 3 \mathrm{H}), 7.17(\mathrm{~d}, J=8.0 \mathrm{~Hz}, 2 \mathrm{H}), 7.00-6.95(\mathrm{~m}, 2 \mathrm{H}), 6.93(\mathrm{~d}, J=0.7 \mathrm{~Hz}, 4 \mathrm{H})$, $6.02(\mathrm{~s}, 1 \mathrm{H}), 5.66(\mathrm{~s}, 1 \mathrm{H}), 4.98(\mathrm{~s}, 1 \mathrm{H}), 4.60(\mathrm{~s}, 1 \mathrm{H}), 3.96(\mathrm{~d}, J=13.7 \mathrm{~Hz}, 1 \mathrm{H}), 3.86(\mathrm{~d}, J=13.7 \mathrm{~Hz}, 1 \mathrm{H}), 2.33$ $(\mathrm{d}, J=7.4 \mathrm{~Hz}, 6 \mathrm{H}) ;{ }^{13} \mathrm{C}$ NMR $\left(126 \mathrm{MHz}, \mathrm{CDCl}_{3}\right) \delta 143.74,143.18,139.77,139.15,135.24,129.38$ (x2), 129.25 (x2), 128.93 (x2), 128.72 (x2), 127.72 (x3), 127.68 (x2), $127.24(x 2), 126.92(x 2), 126.90$ (x2), 126.14 (x2), 100.76, 64.15, 62.85, 46.64, 21.41; HRMS (ESI) calcd for $\left(\mathrm{C}_{31} \mathrm{H}_{31} \mathrm{~N}_{2} \mathrm{O}_{4} \mathrm{~S}_{2}\right)^{+}[\mathrm{M}+\mathrm{H}]^{+}: 559.1720$, found: 559.1710 . 


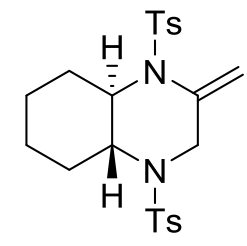

(4aR,8aR)-2-methylene-1,4-ditosyldecahydroquinoxaline (3h): Prepared according to the general method using $1 \mathrm{~h}$ (65 mg, $0.15 \mathrm{mmol}), \mathrm{Pd}_{2}\left(\mathrm{dba}_{3} \cdot \mathrm{CHCl}_{3}(2.4 \mathrm{mg}, 2.3 \mu \mathrm{mol})\right.$, DPEphos (2.7 mg, $\left.5.0 \mu \mathrm{mol}\right)$, propargyl carbonate $2 \mathrm{a}(31 \mathrm{mg}, 0.20 \mathrm{mmol})$ in $\mathrm{CH}_{2} \mathrm{Cl}_{2}(1.5 \mathrm{~mL})$ for $45 \mathrm{~min}$. Purified by flash column chromatography (9:1 to $3: 1$ Hexanes:EtOAc) to afford $3 \mathrm{~h}(67 \mathrm{mg}, 0.15 \mathrm{mmol}, 95 \%$ yield) as a yellow oil.

Analytical data for $3 \mathrm{~h}:{ }^{1} \mathrm{H}$ NMR $\left(500 \mathrm{MHz}, \mathrm{CDCl}_{3}\right) \delta 7.75(\mathrm{~d}, J=8.3 \mathrm{~Hz}, 2 \mathrm{H}), 7.54(\mathrm{~d}, J=8.3 \mathrm{~Hz}, 2 \mathrm{H}), 7.32$ $(\mathrm{dd}, J=8.0,0.6 \mathrm{~Hz}, 2 \mathrm{H}), 7.25(\mathrm{dd}, J=8.0,0.5 \mathrm{~Hz}, 2 \mathrm{H}), 5.35(\mathrm{~d}, J=2.3 \mathrm{~Hz}, 1 \mathrm{H}), 4.99(\mathrm{~d}, J=1.9 \mathrm{~Hz}, 1 \mathrm{H}), 4.04$ (dt, $J=14.3,2.4 \mathrm{~Hz}, 1 \mathrm{H}), 3.85-3.76(\mathrm{~m}, 1 \mathrm{H}), 3.74(\mathrm{~d}, J=14.3 \mathrm{~Hz}, 1 \mathrm{H}), 2.92(\mathrm{td}, J=11.1,3.3 \mathrm{~Hz}, 1 \mathrm{H}), 2.43$ $(\mathrm{s}, 3 \mathrm{H}), 2.40(\mathrm{~s}, 3 \mathrm{H}), 2.37(\mathrm{~d}, J=13.9 \mathrm{~Hz}, 1 \mathrm{H}), 2.05(\mathrm{~d}, J=12.8 \mathrm{~Hz}, 1 \mathrm{H}), 1.76-1.63(\mathrm{~m}, 3 \mathrm{H}), 1.43-1.30(\mathrm{~m}$, 2H), $1.19-1.07(\mathrm{~m}, 1 \mathrm{H}) ;{ }^{13} \mathrm{C} \mathrm{NMR}\left(126 \mathrm{MHz}, \mathrm{CDCl}_{3}\right) \delta 143.91,143.31,138.46,136.53,136.26,129.66$, 129.62 (x2), 128.24, 127.62 (x2), 127.02, 126.86, 113.21, 63.87, 60.08, 51.72, 33.64, 29.66, 25.25, 24.03, 21.53, 21.43.; HRMS (ESI) calcd for $\left(\mathrm{C}_{23} \mathrm{H}_{29} \mathrm{~N}_{2} \mathrm{O}_{4} \mathrm{~S}_{2}\right)^{+}[\mathrm{M}+\mathrm{H}]^{+}: 461.1563$, found: 461.1552 .

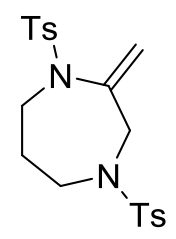

2-methylene-1,4-ditosyl-1,4-diazepane (3i): Prepared according to the general method using $\mathbf{1 i}$ (111 $\mathrm{mg}, 0.3 \mathrm{mmol}), \mathrm{Pd}_{2}\left(\mathrm{dba}_{3} \cdot \mathrm{CHCl}_{3}(4.6 \mathrm{mg}, 4.5 \mu \mathrm{mol})\right.$, DPEphos $(5.3 \mathrm{mg}, 9.9 \mu \mathrm{mol})$, propargyl carbonate $2 \mathrm{a}$ (61 mg, $0.39 \mathrm{mmol}$ ) in $\mathrm{CH}_{2} \mathrm{Cl}_{2}(3.0 \mathrm{~mL}$ ) for $3 \mathrm{~h}$. Purified by flash column chromatography (9:1 to $3: 1$ Hexanes:EtOAc) to afford $\mathbf{3 i}$ ( $110 \mathrm{mg}, 0.27 \mathrm{mmol}, 90 \%$ yield) as an off white solid.

Analytical data for $3 \mathbf{i}:\left(\mathrm{mp}: 144{ }^{\circ} \mathrm{C}\right),{ }^{1} \mathrm{H}$ NMR $\left(500 \mathrm{MHz}, \mathrm{CDCl}_{3}\right) \delta 7.71(\mathrm{dd}, J=8.2,0.9 \mathrm{~Hz}, 2 \mathrm{H}), 7.63(\mathrm{dd}, J=$ $8.2,0.9 \mathrm{~Hz}, 2 \mathrm{H}), 7.36-7.21(\mathrm{~m}, 4 \mathrm{H}), 5.13(\mathrm{~s}, 1 \mathrm{H}), 5.03(\mathrm{~s}, 1 \mathrm{H}), 3.77(\mathrm{~s}, 2 \mathrm{H}), 3.65-3.57(\mathrm{~m}, 2 \mathrm{H}), 3.30-$ $3.21(\mathrm{~m}, 2 \mathrm{H}), 2.42(\mathrm{~s}, 3 \mathrm{H}), 2.41(\mathrm{~s}, 3 \mathrm{H}), 1.94-1.82(\mathrm{~m}, 2 \mathrm{H}) ;{ }^{13} \mathrm{C} \mathrm{NMR}\left(126 \mathrm{MHz}, \mathrm{CDCl}_{3}\right) \delta 143.65,143.42$, 141.34, 136.37 (x2), 135.90, 129.69 (x2), 129.58, 127.41 (x2), 126.98 (x2), 112.73, 53.38, 47.80, 47.59, 28.97, 21.43, 21.38; HRMS (ESI) calcd for $\left(\mathrm{C}_{20} \mathrm{H}_{25} \mathrm{~N}_{2} \mathrm{O}_{4} \mathrm{~S}_{2}\right)^{+}[\mathrm{M}+\mathrm{H}]^{+}: 421.1250$, found: 421.1265 .

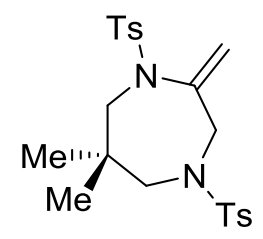

6,6-dimethyl-2-methylene-1,4-ditosyl-1,4-diazepane (3j): Prepared according to the general method using $1 \mathrm{j}$ (82 mg, $0.2 \mathrm{mmol}), \mathrm{Pd}_{2}(\mathrm{dba})_{3} \cdot \mathrm{CHCl}_{3}(3.1 \mathrm{mg}, 3.0 \mu \mathrm{mol})$, DPEphos (3.6 mg, $\left.6.6 \mu \mathrm{mol}\right)$, propargyl carbonate $2 \mathrm{a}$ ( $41 \mathrm{mg}, 0.26 \mathrm{mmol}$ ) in $\mathrm{CH}_{2} \mathrm{Cl}_{2}(2.0 \mathrm{~mL})$ for $1.5 \mathrm{~h}$. Purified by flash column chromatography (9:1 to 3:1 Hexanes:EtOAc) to afford $\mathbf{3 j}$ ( $87 \mathrm{mg}, 0.19 \mathrm{mmol}, 98 \%$ yield) as an off white solid. 
Analytical data for $\mathbf{3 j}$ : (mp: $\left.175^{\circ} \mathrm{C}\right),{ }^{1} \mathrm{H}$ NMR $\left(500 \mathrm{MHz}, \mathrm{CDCl}_{3}\right) \delta 7.65(\mathrm{~d}, \mathrm{~J}=8.0 \mathrm{~Hz}, 2 \mathrm{H}), 7.63(\mathrm{~d}, \mathrm{~J}=7.9$ $\mathrm{Hz}, 2 \mathrm{H}), 7.29(\mathrm{dd}, \mathrm{J}=7.4,6.9 \mathrm{~Hz}, 4 \mathrm{H}), 5.13(\mathrm{~s}, 1 \mathrm{H}), 4.81(\mathrm{~s}, 1 \mathrm{H}), 3.66(\mathrm{~s}, 2 \mathrm{H}), 3.35(\mathrm{~s}, 2 \mathrm{H}), 2.98(\mathrm{~s}, 2 \mathrm{H}), 2.41$ $(\mathrm{s}, 6 \mathrm{H}), 1.03(\mathrm{~s}, 6 \mathrm{H}) ;{ }^{13} \mathrm{C}$ NMR $\left(126 \mathrm{MHz}, \mathrm{CDCl}_{3}\right) \delta 143.74,143.45,140.79,136.13,135.47,129.68$ (x2), 129.57 (x2), 127.32 (x2), 127.06 (x2), 112.42, 58.16, 56.86, 53.50, 36.27, 24.21 (x2), 21.43, 21.39; HRMS (ESI) calcd for $\left(\mathrm{C}_{22} \mathrm{H}_{29} \mathrm{~N}_{2} \mathrm{O}_{4} \mathrm{~S}_{2}\right)+[\mathrm{M}+\mathrm{H}]+:$ 449.1563, found: 449.1584 .

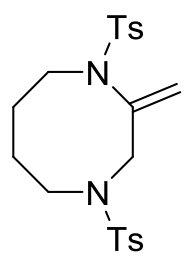

2-methylene-1,4-ditosyl-1,4-diazocane (3k): Prepared according to the general method using $\mathbf{1 k}$ (119 $\mathrm{mg}, 0.3 \mathrm{mmol}), \mathrm{Pd}_{2}(\mathrm{dba})_{3} \cdot \mathrm{CHCl}_{3}(4.6 \mathrm{mg}, 4.5 \mu \mathrm{mol})$, DPEphos (5.3 mg, $\left.9.9 \mu \mathrm{mol}\right)$, propargyl carbonate $2 \mathrm{a}$ (61 mg, $0.39 \mathrm{mmol})$ in $\mathrm{CH}_{2} \mathrm{Cl}_{2}(3.0 \mathrm{~mL})$ for $75 \mathrm{~min}$. Purified by flash column chromatography $(6: 1$ to $4: 1$ Hexanes:EtOAc) to afford $\mathbf{3 k}$ (117 mg, $0.27 \mathrm{mmol}, 90 \%$ yield) as an off white solid.

Analytical data for 3k: (mp: $\left.131{ }^{\circ} \mathrm{C}\right),{ }^{1} \mathrm{H}$ NMR $\left(500 \mathrm{MHz}, \mathrm{CDCl}_{3}\right) \delta 7.66(\mathrm{dd}, J=10.5,8.3 \mathrm{~Hz}, 4 \mathrm{H}), 7.36-$ $7.22(\mathrm{~m}, 4 \mathrm{H}), 5.46(\mathrm{~s}, 1 \mathrm{H}), 4.57(\mathrm{~s}, 1 \mathrm{H}), 3.91(\mathrm{~s}, 2 \mathrm{H}), 3.48(\mathrm{~d}, J=5.5 \mathrm{~Hz}, 2 \mathrm{H}), 3.39(\mathrm{~d}, J=5.1 \mathrm{~Hz}, 2 \mathrm{H}), 2.42$ $(\mathrm{d}, J=2.0 \mathrm{~Hz}, 6 \mathrm{H}), 1.82(\mathrm{~s}, 4 \mathrm{H}) ;{ }^{13} \mathrm{C} \mathrm{NMR}\left(126 \mathrm{MHz}, \mathrm{CDCl}_{3}\right) \delta 143.55,143.22,136.36,129.70(\mathrm{x} 2), 129.44$ (x2), 127.68 (x2), 126.84 (x2), 119.29, 55.63 (x2), 50.78 (x2), 48.38 (x2), 27.91, 22.19, 21.44; HRMS (ESI) calcd for $\left(\mathrm{C}_{21} \mathrm{H}_{27} \mathrm{~N}_{2} \mathrm{O}_{4} \mathrm{~S}_{2}\right)^{+}[\mathrm{M}+\mathrm{H}]^{+}: 435.1407$, found: 435.1415 .<smiles>C=C1CN(C(=O)OCC)c2ccccc2N1C(=O)OCC</smiles>

diethyl 2-methylene-2,3-dihydroquinoxaline-1,4-dicarboxylate (3I): Prepared according to the general method using 11 (50 mg, $0.2 \mathrm{mmol}), \mathrm{Pd}_{2}\left(\mathrm{dba}_{3} \cdot \mathrm{CHCl}_{3}(3.1 \mathrm{mg}, 3.0 \mu \mathrm{mol})\right.$, DPEphos (3.6 mg, $\left.6.6 \mu \mathrm{mol}\right)$, propargyl carbonate $2 \mathrm{a}(41 \mathrm{mg}, 0.26 \mathrm{mmol})$ in $\mathrm{CH}_{2} \mathrm{Cl}_{2}(2.0 \mathrm{~mL})$ for $50 \mathrm{~min}$. Purified by flash column chromatography (9:1 to $3: 1$ Hexanes:EtOAc) to afford 31 (19 mg, $0.07 \mathrm{mmol}, 34 \%$ yield) as a white amorphous solid.

Analytical data for $3 \mathbf{3 :}{ }^{1} \mathrm{H}$ NMR $\left(500 \mathrm{MHz}, \mathrm{CDCl}_{3}\right) \delta 7.55(\mathrm{~s}, 1 \mathrm{H}), 7.41(\mathrm{dd}, J=7.8,1.8 \mathrm{~Hz}, 1 \mathrm{H}), 7.12(\mathrm{pd}, J=$ 7.4, $1.4 \mathrm{~Hz}, 2 \mathrm{H}$ ), $5.38(\mathrm{~s}, 1 \mathrm{H}), 4.93(\mathrm{~s}, 1 \mathrm{H}), 4.46(\mathrm{~d}, J=1.1 \mathrm{~Hz}, 2 \mathrm{H}$ ), 4.26 (qdd, J = 7.1, 3.2, $1.0 \mathrm{~Hz}, 4 \mathrm{H}$ ), 1.32 (qd, $J=7.1,1.0 \mathrm{~Hz}, 6 \mathrm{H}) ;{ }^{13} \mathrm{C} \mathrm{NMR}\left(126 \mathrm{MHz}, \mathrm{CDCl}_{3}\right) \delta 153.42,139.99,133.11,124.97,124.84,124.68$, $124.39,123.79,62.60,62.30,52.59,51.06,14.51,14.27,7.34$; HRMS (ESI) calcd for $\left(\mathrm{C}_{15} \mathrm{H}_{18} \mathrm{~N}_{2} \mathrm{O}_{4} \mathrm{Na}\right)^{+}$ $[\mathrm{M}+\mathrm{Na}]^{+}: 313.1159$, found: 313.1152 . 
<smiles>C=C1CN(c2ccccc2)C(=O)C(=O)N1c1ccccc1</smiles>

5-methylene-1,4-diphenylpiperazine-2,3-dione (4): Prepared according to the general method using SI$1(48 \mathrm{mg}, 0.2 \mathrm{mmol}), \mathrm{Pd}_{2}(\mathrm{dba})_{3} \cdot \mathrm{CHCl}_{3}(3.1 \mathrm{mg}, 3 \mu \mathrm{mol})$, DPEphos (3.6 mg, $\left.6.6 \mu \mathrm{mol}\right)$, propargyl carbonate 2a (41 mg, $0.26 \mathrm{mmol}$ ) in $\mathrm{ClCH}_{2} \mathrm{CH}_{2} \mathrm{Cl}(2 \mathrm{~mL})$ for $18 \mathrm{~h}$ at $80^{\circ} \mathrm{C}$. Purified by flash column chromatography (1:0 to $10: 1 \mathrm{CH}_{2} \mathrm{Cl}_{2} / \mathrm{MeOH}$ ) to afford 4 (30 $\mathrm{mg}, 0.11 \mathrm{mmol}, 54 \%$ yield) as an off white solid.

Analytical data for 4: (mp: $\left.166^{\circ} \mathrm{C}\right),{ }^{1} \mathrm{H}$ NMR $(500 \mathrm{MHz}, \mathrm{MeOD}) \delta 7.37-7.32(\mathrm{~m}, 2 \mathrm{H}), 7.30-7.22(\mathrm{~m}, 5 \mathrm{H})$, $7.16-7.08(\mathrm{~m}, 3 \mathrm{H}), 4.64(\mathrm{~d}, J=0.4 \mathrm{~Hz}, 2 \mathrm{H}), 4.52(\mathrm{~d}, J=1.5 \mathrm{~Hz}, 1 \mathrm{H}), 3.96(\mathrm{~d}, J=1.6 \mathrm{~Hz}, 1 \mathrm{H}) ;{ }^{13} \mathrm{C} \mathrm{NMR}(126$ $\mathrm{MHz}, \mathrm{MeOD}) \delta$ 157.35, 140.94, 138.80, 130.89 (x2), 130.36 (x2), 129.99 (x2), 129.25 (x2), 128.76 (x2), 126.14 (x2), 99.70, 52.92; HRMS (ESI) calcd for $\left(\mathrm{C}_{17} \mathrm{H}_{15} \mathrm{~N}_{2} \mathrm{O}_{2}\right)^{+}[\mathrm{M}+\mathrm{H}]^{+}: 279.1128$, found: 279.1122 .

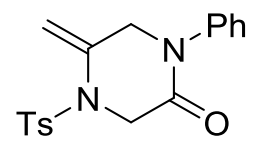

5-methylene-1-phenyl-4-tosylpiperazin-2-one (6a): Prepared according to the general method using 5a (65 mg, $0.15 \mathrm{mmol}$ ), $\mathrm{Pd}_{2}(\mathrm{dba})_{3} \cdot \mathrm{CHCl}_{3}(2.4 \mathrm{mg}, 2.3 \mu \mathrm{mol})$, DPEphos (2.7 mg, $5.0 \mu \mathrm{mol}$ ), propargyl carbonate $2 \mathrm{a}(31 \mathrm{mg}, 0.20 \mathrm{mmol})$ in $\mathrm{CH}_{2} \mathrm{Cl}_{2}(1.5 \mathrm{~mL})$ for $30 \mathrm{~min}$. Purified by flash column chromatography (6:1 to $4: 1$ Hexanes:EtOAc) to afford $6 a$ ( $48 \mathrm{mg}, 0.14 \mathrm{mmol}, 93 \%$ yield) as a white solid.

Analytical data for $6 \mathrm{a}:\left(\mathrm{mp}: 141{ }^{\circ} \mathrm{C}\right),{ }^{1} \mathrm{H}$ NMR $\left(500 \mathrm{MHz}, \mathrm{CDCl}_{3}\right) \delta 7.76(\mathrm{~d}, J=8.3 \mathrm{~Hz}, 2 \mathrm{H}), 7.38$ (qd, $J=9.3$, $7.0 \mathrm{~Hz}, 5 \mathrm{H}), 6.78(\mathrm{dd}, J=8.0,1.1 \mathrm{~Hz}, 2 \mathrm{H}), 4.28(\mathrm{~d}, J=1.1 \mathrm{~Hz}, 1 \mathrm{H}), 4.17(\mathrm{~s}, 2 \mathrm{H}), 4.13(\mathrm{~s}, 2 \mathrm{H}), 3.73(\mathrm{~s}, 1 \mathrm{H})$, 2.48 (s, 3H); ${ }^{13} \mathrm{C}$ NMR (126 MHz, $\left.\mathrm{CDCl}_{3}\right) \delta 163.43,144.60,139.67,136.46,130.02$ (x2), 129.61 (x2), 128.60 (x2), 128.20 (x2), 128.07 (x2), 95.60, 49.75, 47.82, 21.51; HRMS (ESI) calcd for $\left(\mathrm{C}_{18} \mathrm{H}_{19} \mathrm{~N}_{2} \mathrm{O}_{3} \mathrm{~S}\right)^{+}$ $[\mathrm{M}+\mathrm{H}]^{+}: 343.1111$, found: 343.1118 .<smiles>C=C1CN(c2ccc(Cl)cc2)C(=O)CN1[13O]</smiles>

1-(4-chlorophenyl)-5-methylene-4-tosylpiperazin-2-one (6b): Prepared according to the general method using $5 \mathrm{~b}(34 \mathrm{mg}, 0.1 \mathrm{mmol}), \mathrm{Pd}_{2}(\mathrm{dba})_{3} \cdot \mathrm{CHCl}_{3}(1.6 \mathrm{mg}, 1.5 \mu \mathrm{mol})$, DPEphos (1.8 mg, $\left.3.3 \mu \mathrm{mol}\right)$, propargyl carbonate $2 \mathrm{a}(20 \mathrm{mg}, 0.13 \mathrm{mmol})$ in $\mathrm{CH}_{2} \mathrm{Cl}_{2}(1.0 \mathrm{~mL})$ for $90 \mathrm{~min}$. Purified by flash column chromatography (8:1 to 3:1 Hexanes:EtOAc) to afford $\mathbf{6 b}$ (38 mg, $0.1 \mathrm{mmol}, 99 \%$ yield) as a white solid.

Analytical data for $\mathbf{6 b}:\left(\mathrm{mp}: 139{ }^{\circ} \mathrm{C}\right),{ }^{1} \mathrm{H}$ NMR $\left(500 \mathrm{MHz}, \mathrm{CDCl}_{3}\right) \delta 7.75$ (d, J=8.2 Hz, 2H), $7.43-7.29(\mathrm{~m}$, $4 \mathrm{H}), 6.73(\mathrm{~d}, J=8.5 \mathrm{~Hz}, 2 \mathrm{H}), 4.31(\mathrm{~s}, 1 \mathrm{H}), 4.17(\mathrm{~s}, 2 \mathrm{H}), 4.12(\mathrm{~s}, 2 \mathrm{H}), 3.75(\mathrm{~s}, 1 \mathrm{H}), 2.48(\mathrm{~s}, 3 \mathrm{H}) ;{ }^{13} \mathrm{C} \mathrm{NMR}(126$ $\left.\mathrm{MHz}, \mathrm{CDCl}_{3}\right) \delta 163.46,144.65,139.65,134.88,134.59,130.03$ (x2), 129.93 (x2), 129.67 (x3), 128.09 (x2), 95.62, 49.73, 47.76, 21.52; HRMS (ESI) calcd for $\left(\mathrm{C}_{18} \mathrm{H}_{18} \mathrm{CIN}_{2} \mathrm{O}_{3} \mathrm{~S}\right)^{+}[\mathrm{M}+\mathrm{H}]^{+}: 377.0721$, found: 377.0720 . 


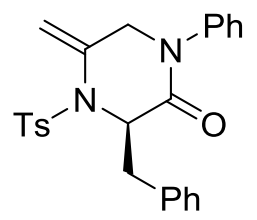

(R)-3-benzyl-5-methylene-1-phenyl-4-tosylpiperazin-2-one (6c): (mp: $140{ }^{\circ} \mathrm{C}$ ), Prepared according to the general method using $5 \mathrm{c}(79 \mathrm{mg}, 0.2 \mathrm{mmol}), \mathrm{Pd}_{2}(\mathrm{dba})_{3} \cdot \mathrm{CHCl}_{3}(3.1 \mathrm{mg}, 3.0 \mu \mathrm{mol})$, DPEphos (3.6 mg, $6.6 \mu \mathrm{mol})$, propargyl carbonate $2 \mathrm{a}(41 \mathrm{mg}, 0.26 \mathrm{mmol})$ in $\mathrm{CH}_{2} \mathrm{Cl}_{2}(2.0 \mathrm{~mL})$ for $45 \mathrm{~min}$. Purified by flash column chromatography (6:1 to $4: 1$ Hexanes:EtOAc) to afford $6 \mathrm{c}(85 \mathrm{mg}, 0.20 \mathrm{mmol}, 98 \%$ yield) as a white solid.

Analytical data for $6 \mathrm{c}:{ }^{1} \mathrm{H}$ NMR $\left(500 \mathrm{MHz}, \mathrm{CDCl}_{3}\right) \delta 7.67(\mathrm{~d}, J=8.3 \mathrm{~Hz}, 2 \mathrm{H}), 7.38-7.22(\mathrm{~m}, 10 \mathrm{H}), 6.56$ (dd, $J=7.8,1.4 \mathrm{~Hz}, 2 \mathrm{H}), 4.80$ (ddd, $J=7.6,4.8,1.7 \mathrm{~Hz}, 1 \mathrm{H}), 4.44(\mathrm{dd}, J=16.9,1.7 \mathrm{~Hz}, 1 \mathrm{H}), 4.08(\mathrm{~s}, 1 \mathrm{H}), 3.82(\mathrm{~d}$, $J=16.8 \mathrm{~Hz}, 1 \mathrm{H}), 3.50(\mathrm{~s}, 1 \mathrm{H}), 3.39(\mathrm{dd}, J=14.0,7.7 \mathrm{~Hz}, 1 \mathrm{H}), 3.29(\mathrm{dd}, J=14.0,4.8 \mathrm{~Hz}, 1 \mathrm{H}), 2.45(\mathrm{~s}, 3 \mathrm{H}) ;{ }^{13} \mathrm{C}$ NMR $\left(126 \mathrm{MHz}_{2} \mathrm{CDCl}_{3}\right) \delta 165.84,144.03,140.01,136.70,136.19,129.71,129.70$ (x2), 129.45, 129.43 (x3), 128.58 (x2), 128.39, 128.00 (x2), 127.80 (x2), 127.13, 94.69, 59.28, 43.57, 38.15, 21.41; HRMS (ESI) calcd for $\left(\mathrm{C}_{25} \mathrm{H}_{25} \mathrm{~N}_{2} \mathrm{O}_{3} \mathrm{~S}\right)^{+}[\mathrm{M}+\mathrm{H}]^{+}: 433.1580$, found: 433.1586 .<smiles>[3H]N1C(=C)CN(c2ccccc2)C(=O)[C@@H]1C</smiles>

(S)-3-methyl-5-methylene-1-phenyl-4-tosylpiperazin-2-one (6d): $\left(\mathrm{mp}: 131^{\circ} \mathrm{C}\right)$, Prepared according to a modification of the general method using $5 \mathbf{d}(20 \mathrm{mg}, 0.07 \mathrm{mmol})$. To this $1 \mathrm{~mL}$ of a prepared stock solution $\left(\mathrm{Pd}_{2}(\mathrm{dba})_{3} \cdot \mathrm{CHCl}_{3}(3.1 \mathrm{mg}, 3.0 \mu \mathrm{mol})\right.$, DPEphos $(3.6 \mathrm{mg}, 6.6 \mu \mathrm{mol})$ in $\left.\mathrm{CH}_{2} \mathrm{Cl}_{2}(3 \mathrm{~mL})\right)$ was added followed by propargyl carbonate $2 \mathrm{a}(14 \mathrm{mg}, 0.09 \mathrm{mmol})$ and stirred for $4 \mathrm{~h}$. Purified by flash column chromatography (6:1 to 4:1 Hexanes:EtOAc) to afford $6 \mathrm{~d}(22 \mathrm{mg}, 0.07 \mathrm{mmol}, 98 \%$ yield) as a white solid.

Analytical data for $6 \mathrm{~d}:{ }^{1} \mathrm{H}$ NMR $\left(500 \mathrm{MHz}, \mathrm{CDCl}_{3}\right) \delta 7.79(\mathrm{~d}, J=8.3 \mathrm{~Hz}, 2 \mathrm{H}), 7.39-7.29(\mathrm{~m}, 5 \mathrm{H}), 6.47(\mathrm{~d}, J$ $=5.4 \mathrm{~Hz}, 2 \mathrm{H}), 4.70(\mathrm{qd}, J=7.2,1.8 \mathrm{~Hz}, 1 \mathrm{H}), 4.63(\mathrm{dd}, J=16.9,1.6 \mathrm{~Hz}, 1 \mathrm{H}), 4.29(\mathrm{~d}, J=16.8 \mathrm{~Hz}, 1 \mathrm{H}), 4.19$ (s, $1 \mathrm{H}), 3.55(\mathrm{~s}, 1 \mathrm{H}), 2.49(\mathrm{~s}, 3 \mathrm{H}), 1.66(\mathrm{~d}, J=7.2 \mathrm{~Hz}, 3 \mathrm{H}) ;{ }^{13} \mathrm{C} \mathrm{NMR}\left(126 \mathrm{MHz}, \mathrm{CDCl}_{3}\right) \delta$ 166.95, 144.16, 139.88, 136.57, 135.99, 129.89 (x2), 129.44 (x2), 128.40, 128.10 (x2), 127.85 (x2), 94.55, 54.37, 42.72, 21.46, 17.84; HRMS (ESI) calcd for $\left(\mathrm{C}_{19} \mathrm{H}_{21} \mathrm{~N}_{2} \mathrm{O}_{3} \mathrm{~S}\right)^{+}[\mathrm{M}+\mathrm{H}]^{+}$: 357.1267, found: 357.1271.<smiles>C=C1CN(c2ccc(Cl)cc2)C(=O)[C@H](C)N1[13CH3]</smiles>

(S)-1-(4-chlorophenyl)-3-methyl-5-methylene-4-tosylpiperazin-2-one (6e): Prepared according to the general method using $5 \mathrm{e}(16 \mathrm{mg}, 0.05 \mathrm{mmol}), \mathrm{Pd}_{2}\left(\mathrm{dba}_{3} \cdot \mathrm{CHCl}_{3}(0.8 \mathrm{mg}, 0.8 \mu \mathrm{mol})\right.$, DPEphos (0.9 mg, 1.6 $\mu \mathrm{mol})$, propargyl carbonate $2 \mathrm{a}(10 \mathrm{mg}, 0.07 \mathrm{mmol})$ in $\mathrm{CH}_{2} \mathrm{Cl}_{2}(0.5 \mathrm{~mL})$ for $60 \mathrm{~min}$. Purified by flash 
column chromatography (8:1 to $3: 1$ Hexanes:EtOAc) to afford 6 e $(21 \mathrm{mg}, 0.05 \mathrm{mmol}, 98 \%$ yield) as a white solid.

Analytical data for $6 \mathrm{e}:\left(\mathrm{mp}: 145^{\circ} \mathrm{C}\right),{ }^{1} \mathrm{H}$ NMR $\left(500 \mathrm{MHz}, \mathrm{CDCl}_{3}\right) \delta 7.77(\mathrm{~d}, J=7.9 \mathrm{~Hz}, 2 \mathrm{H}), 7.33$ (dd, $J=7.9$, $0.6 \mathrm{~Hz}, 2 \mathrm{H}$ ), 7.29 (dd, $J=8.8,0.8 \mathrm{~Hz}, 2 \mathrm{H}), 6.42(\mathrm{~d}, J=8.3 \mathrm{~Hz}, 2 \mathrm{H}$ ), 4.69 (dddd, $J=8.2,7.2,6.4,1.0 \mathrm{~Hz}, 1 \mathrm{H}$ ), $4.66-4.57(\mathrm{~m}, 1 \mathrm{H}), 4.27(\mathrm{dd}, J=16.8,0.8 \mathrm{~Hz}, 1 \mathrm{H}), 4.21(\mathrm{~s}, 1 \mathrm{H}), 3.56(\mathrm{~d}, J=0.8 \mathrm{~Hz}, 1 \mathrm{H}), 2.48(\mathrm{~s}, 3 \mathrm{H}), 1.64$ $(\mathrm{dd}, J=7.2,0.8 \mathrm{~Hz}, 3 \mathrm{H}) ;{ }^{13} \mathrm{C} \mathrm{NMR}\left(126 \mathrm{MHz}, \mathrm{CDCl}_{3}\right) \delta 166.98,144.33,139.69,135.90,135.47,134.96$, 134.38, 129.89, 129.75 (x2), 129.56 (x2), 127.88 (x2), 94.59, 54.33, 42.61, 21.48, 17.75; HRMS (ESI) calcd for $\left(\mathrm{C}_{19} \mathrm{H}_{20} \mathrm{ClN}_{2} \mathrm{O}_{3} \mathrm{~S}\right)^{+}[\mathrm{M}+\mathrm{H}]^{+}$: 391.0878 , found: 391.0879 .<smiles>C=C1CN(c2ccc([N+](=O)[O-])cc2)C(=O)[C@H](C)N1[As]</smiles>

(S)-3-methyl-5-methylene-1-(4-nitrophenyl)-4-tosylpiperazin-2-one (6f): Prepared according to the general method using $5 \mathbf{f}(33 \mathrm{mg}, 0.1 \mathrm{mmol}), \mathrm{Pd}_{2}\left(\mathrm{dba}_{3} \cdot \mathrm{CHCl}_{3}(1.6 \mathrm{mg}, 1.5 \mu \mathrm{mol})\right.$, DPEphos $(1.8 \mathrm{mg}, 3.3$ umol), propargyl carbonate $2 \mathrm{a}(20 \mathrm{mg}, 0.13 \mathrm{mmol})$ in $\mathrm{CH}_{2} \mathrm{Cl}_{2}(1.0 \mathrm{~mL})$ for $60 \mathrm{~min}$. Purified by flash column chromatography ( $8: 1$ to $3: 1$ Hexanes:EtOAc) to afford $6 f(35 \mathrm{mg}, 0.1 \mathrm{mmol}, 97 \%$ yield) as a white solid.

Analytical data for $6 \mathbf{6 f}$ ( $\left.\mathrm{mp}: 112{ }^{\circ} \mathrm{C}\right),{ }^{1} \mathrm{H}$ NMR $\left(500 \mathrm{MHz}, \mathrm{CDCl}_{3}\right) \delta 8.20(\mathrm{~d}, J=9.0 \mathrm{~Hz}, 2 \mathrm{H}), 7.79(\mathrm{~d}, J=8.3$ $\mathrm{Hz}, 2 \mathrm{H}), 7.35(\mathrm{~d}, J=8.1 \mathrm{~Hz}, 2 \mathrm{H}), 6.71(\mathrm{~d}, J=8.9 \mathrm{~Hz}, 2 \mathrm{H}), 4.72(\mathrm{qd}, J=7.2,1.7 \mathrm{~Hz}, 1 \mathrm{H}), 4.66(\mathrm{~d}, J=17.1 \mathrm{~Hz}$, $1 \mathrm{H}), 4.30(\mathrm{~m}, 1 \mathrm{H}), 4.28(\mathrm{~d}, J=1.4 \mathrm{~Hz}, 1 \mathrm{H}), 3.54(\mathrm{~s}, 1 \mathrm{H}) 2.51(\mathrm{~s}, 3 \mathrm{H}), 1.65(\mathrm{~d}, J=7.2 \mathrm{~Hz}, 3 \mathrm{H}) ;{ }^{13} \mathrm{C}$ NMR $(126$ $\left.\mathrm{MHz}, \mathrm{CDCl}_{3}\right) \delta 166.90,147.43,144.40,142.35,139.51,135.86,129.93$ (x2), 129.60 (x2), 127.89 (x2), 124.79 (x2), 94.89, 54.29, 42.54, 21.51, 17.63; HRMS (ESI) calcd for $\left(\mathrm{C}_{19} \mathrm{H}_{20} \mathrm{~N}_{3} \mathrm{O}_{5} \mathrm{~S}\right)^{+}[\mathrm{M}+\mathrm{H}]^{+}: 402.1118$, found: 402.1118.<smiles>C=C1CN(c2cc(C(F)(F)F)cc(C(F)(F)F)c2)C(=O)[C@H](C)N1[Hg]</smiles>

(S)-1-(3,5-bis(trifluoromethyl)phenyl)-3-methyl-5-methylene-4-tosylpiperazin-2-one (6g): Prepared according to the general method using $5 \mathrm{~g}(59 \mathrm{mg}, 0.15 \mathrm{mmol}), \mathrm{Pd}_{2}(\mathrm{dba})_{3} \cdot \mathrm{CHCl}_{3}(2.3 \mathrm{mg}, 2.2 \mu \mathrm{mol})$, DPEphos (2.4 mg, $4.5 \mu \mathrm{mol}$ ), propargyl carbonate $2 \mathrm{a}(31 \mathrm{mg}, 0.20 \mathrm{mmol})$ in $\mathrm{CH}_{2} \mathrm{Cl}_{2}(1.5 \mathrm{~mL})$ for $75 \mathrm{~min}$. Purified by flash column chromatography ( $8: 1$ to $3: 1$ Hexanes:EtOAc) to afford $6 \mathrm{~g} \mathrm{(39} \mathrm{mg}, 0.09 \mathrm{mmol}$, $62 \%$ yield) as a white solid.

Analytical data for $6 \mathrm{~g}:\left(\mathrm{mp}: 143{ }^{\circ} \mathrm{C}\right),{ }^{1} \mathrm{H}$ NMR $\left(500 \mathrm{MHz}, \mathrm{CDCl}_{3}\right) \delta 7.84(\mathrm{~s}, 1 \mathrm{H}), 7.79(\mathrm{~d}, J=8.3 \mathrm{~Hz}, 2 \mathrm{H}), 7.35$ $(\mathrm{d}, J=8.4 \mathrm{~Hz}, 2 \mathrm{H}), 6.99(\mathrm{~s}, 2 \mathrm{H}), 4.77(\mathrm{qd}, J=7.2,1.7 \mathrm{~Hz}, 1 \mathrm{H}), 4.69(\mathrm{dd}, J=17.1,1.7 \mathrm{~Hz}, 1 \mathrm{H}), 4.33(\mathrm{dt}, J=$ 
17.0, $1.7 \mathrm{~Hz}, 1 \mathrm{H}), 4.28(\mathrm{~d}, J=1.8 \mathrm{~Hz}, 1 \mathrm{H}), 3.46(\mathrm{~d}, J=1.9 \mathrm{~Hz}, 1 \mathrm{H}), 2.46(\mathrm{~s}, 3 \mathrm{H}), 1.69(\mathrm{~d}, J=7.3 \mathrm{~Hz}, 3 \mathrm{H}) ;{ }^{13} \mathrm{C}$ NMR $\left(126 \mathrm{MHz}_{\mathrm{CDCl}}\right.$ ) $\delta 167.00,144.79,139.64,137.96,133.28,133.00,129.92$ (x2), 129.17, 127.92 (x2), $127.33(x 2), 123.69,122.49,121.52,94.80,54.30,42.50,21.29,17.78$; HRMS (ESI) calcd for $\left(\mathrm{C}_{21} \mathrm{H}_{19} \mathrm{~F}_{6} \mathrm{~N}_{2} \mathrm{O}_{3} \mathrm{~S}\right)^{+}[\mathrm{M}+\mathrm{H}]^{+}: 493.1015$, found: 493.1016 .<smiles>C=C1CN(c2cc(C)cc(C)c2)C(=O)[C@H](C)N1[Te]</smiles>

(S)-1-(3,5-dimethylphenyl)-3-methyl-5-methylene-4-tosylpiperazin-2-one (6h): Prepared according to the general method using $1 \mathrm{~h}(23 \mathrm{mg}, 0.07 \mathrm{mmol}), \mathrm{Pd}_{2}\left(\mathrm{dba}_{3} \cdot \mathrm{CHCl}_{3}(1.1 \mathrm{mg}, 1.0 \mu \mathrm{mol})\right.$, DPEphos (1.2 $\mathrm{mg}$, $2.2 \mu \mathrm{mol})$, propargyl carbonate $2 \mathrm{a}(14 \mathrm{mg}, 0.09 \mathrm{mmol})$ in $\mathrm{CH}_{2} \mathrm{Cl}_{2}(1.0 \mathrm{~mL})$ for $5 \mathrm{~h}$. Purified by flash column chromatography (4:1 to 3:1 Hexanes:EtOAc) to afford $6 \mathrm{~h}$ ( $15 \mathrm{mg}, 0.04 \mathrm{mmol}, 61 \%$ yield) as a white solid.

Analytical data for $6 \mathrm{~h}:\left(\mathrm{mp}: 169^{\circ} \mathrm{C}\right),{ }^{1} \mathrm{H}$ NMR $\left(500 \mathrm{MHz}, \mathrm{CDCl}_{3}\right) \delta 7.74(\mathrm{~d}, J=8.3 \mathrm{~Hz}, 2 \mathrm{H}), 7.34(\mathrm{~d}, J=8.0$ $\mathrm{Hz}, 2 \mathrm{H}), 6.90(\mathrm{~s}, 1 \mathrm{H}), 6.63$ (br. s, 1H), $5.90(\mathrm{~s}, 1 \mathrm{H}), 5.46(\mathrm{~s}, 1 \mathrm{H}), 4.73(\mathrm{tt}, J=7.1,3.5 \mathrm{~Hz}, 1 \mathrm{H}), 2.45(\mathrm{~s}, 3 \mathrm{H})$, $2.25(\mathrm{~s}, 3 \mathrm{H}), 2.21(\mathrm{~s}, 3 \mathrm{H}), 1.52(\mathrm{~d}, J=1.1 \mathrm{~Hz}, 3 \mathrm{H}), 1.46(\mathrm{~d}, J=7.1 \mathrm{~Hz}, 3 \mathrm{H}) ;{ }^{13} \mathrm{C} \mathrm{NMR}\left(126 \mathrm{MHz}, \mathrm{CDCl}_{3}\right) \delta$ 165.91, 143.84, 136.04, 130.15 (x2), 129.70 (x4), 127.23 (x4), 103.89 (x2), 54.46 (x2), 21.58, 21.10, 16.76, 16.73; HRMS (ESI) calcd for $\left(\mathrm{C}_{21} \mathrm{H}_{25} \mathrm{~N}_{2} \mathrm{O}_{3} \mathrm{~S}\right)^{+}[\mathrm{M}+\mathrm{H}]^{+}: 385.1580$, found: 385.1581 .<smiles>C=C1CN(c2ccc(OC)cc2)C(=O)[C@H](C)N1[TeH]</smiles>

(S)-1-(4-methoxyphenyl)-3-methyl-5-methylene-4-tosylpiperazin-2-one (6i): Prepared according to the general method using $5 \mathrm{i}(32 \mathrm{mg}, 0.1 \mathrm{mmol}), \mathrm{Pd}_{2}(\mathrm{dba})_{3} \cdot \mathrm{CHCl}_{3}(1.6 \mathrm{mg}, 1.5 \mu \mathrm{mol})$, DPEphos (1.8 mg, 3.3 $\mu \mathrm{mol})$, propargyl carbonate $2 \mathrm{a}(20 \mathrm{mg}, 0.13 \mathrm{mmol})$ in $\mathrm{CH}_{2} \mathrm{Cl}_{2}(1.0 \mathrm{~mL})$ for $5 \mathrm{~h}$. Purified by flash column chromatography (8:1 to 3:1 Hexanes:EtOAc) to afford $6 \mathbf{i}(34 \mathrm{mg}, 0.1 \mathrm{mmol}, 98 \%$ yield) as a white solid.

Analytical data for $\mathbf{6 i}$ : $\left(\mathrm{mp}: 116{ }^{\circ} \mathrm{C}\right),{ }^{1} \mathrm{H}$ NMR $\left(500 \mathrm{MHz}, \mathrm{CDCl}_{3}\right) \delta 7.78(\mathrm{~d}, J=8.3 \mathrm{~Hz}, 2 \mathrm{H}), 7.33(\mathrm{~d}, J=8.0$ $\mathrm{Hz}, 2 \mathrm{H}), 6.82(\mathrm{~d}, J=9.1 \mathrm{~Hz}, 2 \mathrm{H}), 6.39(\mathrm{~d}, J=8.2 \mathrm{~Hz}, 2 \mathrm{H}), 4.69(\mathrm{tt}, J=7.2,3.6 \mathrm{~Hz}, 1 \mathrm{H}), 4.62(\mathrm{dd}, J=16.9,1.8$ $\mathrm{Hz}, 1 \mathrm{H}), 4.28(\mathrm{dt}, J=16.8,1.8 \mathrm{~Hz}, 1 \mathrm{H}), 4.18(\mathrm{~s}, 1 \mathrm{H}), 3.78(\mathrm{~s}, 3 \mathrm{H}), 3.59(\mathrm{t}, J=1.6 \mathrm{~Hz}, 1 \mathrm{H}), 2.48(\mathrm{~s}, 3 \mathrm{H}), 1.65$ $(\mathrm{d}, J=7.2 \mathrm{~Hz}, 3 \mathrm{H}) ;{ }^{13} \mathrm{C} \mathrm{NMR}\left(126 \mathrm{MHz}, \mathrm{CDCl}_{3}\right) \delta 167.13,159.22,144.08,139.98,135.84,129.84(\mathrm{x} 2)$, 129.00 (x2), 127.82 (x2), 114.68 (x2), 94.41, 55.37 (x2), 54.36, 42.68, 21.45, 17.81; HRMS (ESI) calcd for $\left(\mathrm{C}_{20} \mathrm{H}_{23} \mathrm{~N}_{2} \mathrm{O}_{4} \mathrm{~S}\right)^{+}[\mathrm{M}+\mathrm{H}]^{+}: 387.1373$, found: 387.1378 . 


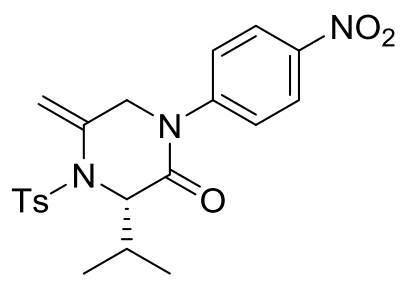

(S)-3-isopropyl-5-methylene-1-(4-nitrophenyl)-4-tosylpiperazin-2-one (6j): Prepared according to the general method using $5 \mathrm{j}$ (117 mg, $0.3 \mathrm{mmol}$ ), $\mathrm{Pd}_{2}(\mathrm{dba})_{3} \cdot \mathrm{CHCl}_{3}(4.6 \mathrm{mg}, 4.5 \mu \mathrm{mol})$, DPEphos (5.3 mg, 9.9 umol), propargyl carbonate $2 \mathrm{a}(61 \mathrm{mg}, 0.39 \mathrm{mmol})$ in $\mathrm{CH}_{2} \mathrm{Cl}_{2}(3.0 \mathrm{~mL})$ for $36 \mathrm{~h}$. Purified by flash column chromatography (6:1 to 2:1 Hexanes:EtOAc) to afford $6 \mathbf{j}(110 \mathrm{mg}, 0.26 \mathrm{mmol}, 85 \%$ yield) as a yellow oil.

Analytical data for $6 \mathbf{6 j}:{ }^{1} \mathrm{H}$ NMR $\left(500 \mathrm{MHz}, \mathrm{CDCl}_{3}\right) \delta 8.17(\mathrm{~d}, J=9.0 \mathrm{~Hz}, 2 \mathrm{H}), 7.78(\mathrm{~d}, J=8.2 \mathrm{~Hz}, 2 \mathrm{H}), 7.35$ (d, $J=8.3 \mathrm{~Hz}, 2 \mathrm{H}), 6.61(\mathrm{~d}, J=8.9 \mathrm{~Hz}, 2 \mathrm{H}), 4.72(\mathrm{~d}, J=17.5 \mathrm{~Hz}, 1 \mathrm{H}), 4.29(\mathrm{~d}, J=17.5 \mathrm{~Hz}, 1 \mathrm{H}), 4.24-4.16(\mathrm{~m}$, $2 \mathrm{H}), 3.51(\mathrm{~s}, 1 \mathrm{H}), 2.50(\mathrm{~s}, 3 \mathrm{H}), 2.39-2.24(\mathrm{~m}, 1 \mathrm{H}), 1.17(\mathrm{t}, J=7.1 \mathrm{~Hz}, 6 \mathrm{H}) ;{ }^{13} \mathrm{C} \mathrm{NMR}\left(126 \mathrm{MHz}, \mathrm{CDCl}_{3}\right) \delta$ 165.29, 147.31, 144.30, 142.60, 139.62, 135.95, 129.93 (x2), 129.56 (x2), 127.89 (x2), 124.69 (x2), 94.50, $63.73,43.36,29.91,21.50,20.01,19.52$; HRMS (ESI) calcd for $\left(\mathrm{C}_{21} \mathrm{H}_{24} \mathrm{~N}_{3} \mathrm{O}_{5} \mathrm{~S}\right)^{+}[\mathrm{M}+\mathrm{H}]^{+}: 430.1431$, found: 430.1411 .

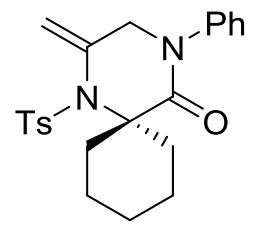

2-methylene-4-phenyl-1-tosyl-1,4-diazaspiro[5.5]undecan-5-one (6k): Prepared according to the general method using 5k (37 mg, $0.1 \mathrm{mmol}), \mathrm{Pd}_{2}(\mathrm{dba})_{3} \cdot \mathrm{CHCl}_{3}(1.6 \mathrm{mg}, 1.5 \mu \mathrm{mol})$, DPEphos (1.8 mg, 3.3 $\mu \mathrm{mol})$, propargyl carbonate $2 \mathrm{a}(20 \mathrm{mg}, 0.13 \mathrm{mmol})$ in $\mathrm{CH}_{2} \mathrm{Cl}_{2}(1.0 \mathrm{~mL})$ for $100 \mathrm{~min}$. Purified by flash column chromatography (9:1 to $4: 1 \mathrm{Hex}$ :EtOAc) to afford $6 \mathbf{k}(40 \mathrm{mg}, 0.1 \mathrm{mmol}, 98 \%$ yield) as a white solid.

Analytical data for 6k: (mp: $\left.138{ }^{\circ} \mathrm{C}\right),{ }^{1} \mathrm{H}$ NMR $\left(500 \mathrm{MHz}, \mathrm{CDCl}_{3}\right) \delta 7.79(\mathrm{~d}, J=7.1 \mathrm{~Hz}, 2 \mathrm{H}), 7.31(\mathrm{dd}, J=$ 13.6, $5.3 \mathrm{~Hz}, 5 \mathrm{H}), 6.54(\mathrm{~d}, J=7.8 \mathrm{~Hz}, 2 \mathrm{H}), 4.75(\mathrm{~d}, J=1.3 \mathrm{~Hz}, 2 \mathrm{H}), 4.15-4.09(\mathrm{~m}, 1 \mathrm{H}), 3.62-3.57(\mathrm{~m}, 1 \mathrm{H})$, $2.47(\mathrm{~s}, 5 \mathrm{H}), 2.07-2.00(\mathrm{~m}, 2 \mathrm{H}), 1.72-1.59(\mathrm{~m}, 5 \mathrm{H}), 1.47-1.29(\mathrm{~m}, 1 \mathrm{H}) ;{ }^{13} \mathrm{C} \mathrm{NMR}\left(126 \mathrm{MHz}, \mathrm{CDCl}_{3}\right) \delta$ 169.04, 143.91, 141.17, 137.47, 129.80 (x2), 129.23 (x2), 128.27 (x3), 127.95 (x2), 92.42, 63.84, 45.62 (x2), 33.32 (x2), 25.42, 21.45, 21.32; HRMS (ESI) calcd for $\left(\mathrm{C}_{23} \mathrm{H}_{26} \mathrm{~N}_{2} \mathrm{O}_{3} \mathrm{SNa}\right)^{+}[\mathrm{M}+\mathrm{Na}]^{+}: 433.1556$, found: 433.1560 . 


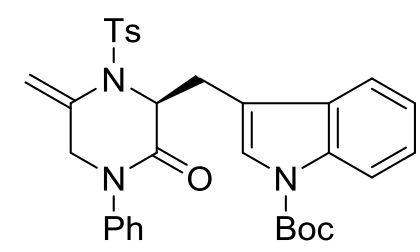

tert-butyl (S)-3-((6-methylene-3-oxo-4-phenyl-1-tosylpiperazin-2-yl)methyl)-1H-indole-1-carboxylate (6I): Prepared according to the general method using 51 (32 $\mathrm{mg}, 0.06 \mathrm{mmol}$ ), palladium and phosphine added as a solution $\left(0.5 \mathrm{~mL}\right.$ of $\mathrm{Pd}_{2}(\mathrm{dba})_{3} \cdot \mathrm{CHCl}_{3}(3.6 \mathrm{mg}, 3.6 \mu \mathrm{mol})$, DPEphos $(4.2 \mathrm{mg}, 8.0 \mu \mathrm{mol})$ in $2.0 \mathrm{~mL}$ $\mathrm{CH}_{2} \mathrm{Cl}_{2}$ ), propargyl carbonate $2 \mathrm{a}(13 \mathrm{mg}, 0.08 \mathrm{mmol})$ in $\mathrm{CH}_{2} \mathrm{Cl}_{2}(0.5 \mathrm{~mL})$ for $3.5 \mathrm{~h}$. Purified by flash column chromatography (9:1 to 4:1 Hex:EtOAc) to afford 61 (33 mg, $0.06 \mathrm{mmol}, 97 \%$ yield) as a white solid.

Analytical data for $6 \mathrm{I}:\left(\mathrm{mp}: 107^{\circ} \mathrm{C}\right),{ }^{1} \mathrm{H}$ NMR $\left(500 \mathrm{MHz}, \mathrm{CDCl}_{3}\right) \delta 8.13(\mathrm{~s}, 1 \mathrm{H}), 7.64(\mathrm{~d}, J=8.3 \mathrm{~Hz}, 2 \mathrm{H}), 7.58$ $(\mathrm{d}, J=7.8 \mathrm{~Hz}, 1 \mathrm{H}), 7.54(\mathrm{~s}, 1 \mathrm{H}), 7.39-7.27(\mathrm{~m}, 4 \mathrm{H}), 7.24(\mathrm{t}, J=7.1 \mathrm{~Hz}, 1 \mathrm{H}), 7.18(\mathrm{~d}, J=8.0 \mathrm{~Hz}, 2 \mathrm{H}), 6.59$ $(\mathrm{d}, J=6.1 \mathrm{~Hz}, 2 \mathrm{H}), 4.87-4.78(\mathrm{~m}, 1 \mathrm{H}), 4.50(\mathrm{dd}, J=16.7,1.6 \mathrm{~Hz}, 1 \mathrm{H}), 4.11(\mathrm{~s}, 1 \mathrm{H}), 3.94(\mathrm{~d}, J=16.7 \mathrm{~Hz}$, $1 \mathrm{H}), 3.53(\mathrm{~s}, 1 \mathrm{H}), 3.46(\mathrm{dd}, J=14.9,7.3 \mathrm{~Hz}, 1 \mathrm{H}), 3.39(\mathrm{dd}, J=14.9,4.6 \mathrm{~Hz}, 1 \mathrm{H}), 2.42(\mathrm{~s}, 3 \mathrm{H}), 1.69(\mathrm{~s}, 9 \mathrm{H})$; ${ }^{13} \mathrm{C} \mathrm{NMR}\left(126 \mathrm{MHz}, \mathrm{CDCl}_{3}\right) \delta 166.23,149.47,144.10,140.03,136.86,135.39,130.10,129.56$ (x2), 129.51, 128.47 (x2), 128.15, 127.85 (x2), 124.67, 124.56, 122.76, 119.14, 115.24, 115.20, 94.88, 83.67, 58.37, 43.77, 31.57, 28.25 (x3), 22.63, 21.51, 14.08; HRMS (ESI) calcd for $\left(\mathrm{C}_{32} \mathrm{H}_{34} \mathrm{~N}_{3} \mathrm{O}_{5} \mathrm{~S}\right)^{+}[\mathrm{M}+\mathrm{H}]^{+}: 572.2214$, found: 572.2208 .<smiles>C=C1CN(c2ccccc2)C(=O)[C@@H](CO[Na])N1[Hg]</smiles>

(R)-3-(((tert-butyldimethylsilyl)oxy)methyl)-5-methylene-1-phenyl-4-tosylpiperazin-2-one

$(6 \mathrm{~m}):$

Prepared according to the general method using $5 \mathrm{~m}(16 \mathrm{mg}, 0.05 \mathrm{mmol})$, palladium and phosphine added as a solution $\left(0.5 \mathrm{~mL}\right.$ of $\mathrm{Pd}_{2}(\mathrm{dba})_{3} \cdot \mathrm{CHCl}_{3}(3.2 \mathrm{mg}, 3.2 \mu \mathrm{mol})$, DPEphos (3.6 mg, $\left.3.2 \mu \mathrm{mol}\right)$ in $2.0 \mathrm{~mL}$ $\mathrm{CH}_{2} \mathrm{Cl}_{2}$ ), propargyl carbonate 2a (13 mg, $\left.0.08 \mathrm{mmol}\right)$ in $\mathrm{CH}_{2} \mathrm{Cl}_{2}(0.5 \mathrm{~mL})$ for $5 \mathrm{~h}$. Purified by flash column chromatography (9:1 to 4:1 Hex:EtOAc) to afford $6 \mathrm{~m}(17 \mathrm{mg}, 0.05 \mathrm{mmol}, 97 \%$ yield) as a white solid.

Analytical data for $6 \mathrm{~m}$ : (mp: $\left.117^{\circ} \mathrm{C}\right),{ }^{1} \mathrm{H}$ NMR $\left(500 \mathrm{MHz}, \mathrm{CDCl}_{3}\right) \delta 7.77(\mathrm{~d}, J=8.2 \mathrm{~Hz}, 2 \mathrm{H}), 7.33(\mathrm{~d}, J=7.6$ $\mathrm{Hz}, 5 \mathrm{H}), 6.52(\mathrm{~s}, 2 \mathrm{H}), 4.87(\mathrm{~d}, J=16.3 \mathrm{~Hz}, 1 \mathrm{H}), 4.62(\mathrm{dd}, J=16.4,1.3 \mathrm{~Hz}, 1 \mathrm{H}), 4.53(\mathrm{~d}, J=2.0 \mathrm{~Hz}, 1 \mathrm{H}), 4.37$ $(\mathrm{dd}, J=10.2,3.0 \mathrm{~Hz}, 1 \mathrm{H}), 4.10(\mathrm{dd}, J=10.2,2.2 \mathrm{~Hz}, 1 \mathrm{H}), 3.50(\mathrm{~s}, 1 \mathrm{H}), 2.48(\mathrm{~s}, 3 \mathrm{H}), 0.89(\mathrm{~s}, 9 \mathrm{H}), 0.08(\mathrm{~s}$, 3H), 0.05 (s, 3H); ${ }^{13} \mathrm{C}$ NMR (126 MHz, CDCl 3 ) $\delta 164.90,144.06,140.59,136.99,136.16,129.86$ (x2), 129.45 (x2), 128.36), 128.17 (x2), 127.82 (x2, 94.14, 67.31, 60.07, 46.10, 25.75 (x3), 21.47, 18.06, -5.67, 5.72; HRMS (ESI) calcd for $\left(\mathrm{C}_{25} \mathrm{H}_{34} \mathrm{~N}_{2} \mathrm{O}_{4} \mathrm{SSiNa}\right)^{+}[\mathrm{M}+\mathrm{Na}]^{+}: 509.1901$, found: 509.1904 . 
<smiles>C=C1CN(c2ccc(C(F)(F)F)cc2)C(=O)[C@@H](Cc2ccccc2)N1[N+](=O)[O-]</smiles>

(R)-3-benzyl-5-methylene-4-((4-nitrophenyl)sulfonyl)-1-(4-(trifluoromethyl)phenyl)piperazin-2-one (6n): Prepared according to the general method using $5 \mathrm{n}(109 \mathrm{mg}, 0.22 \mathrm{mmol}), \mathrm{Pd}_{2}(\mathrm{dba})_{3} \cdot \mathrm{CHCl}_{3}(3.4 \mathrm{mg}$, $3.3 \mu \mathrm{mol})$, DPEphos $(3.9 \mathrm{mg}, 7.3 \mu \mathrm{mol})$, propargyl carbonate $2 \mathrm{a}(45 \mathrm{mg}, 0.29 \mathrm{mmol})$ in $\mathrm{CH}_{2} \mathrm{Cl}_{2}(2.2 \mathrm{~mL})$ for $24 \mathrm{~h}$. Purified by flash column chromatography (1:1 to $1: 0 \mathrm{CH}_{2} \mathrm{Cl}_{2}$ :Hexanes) to afford $6 \mathrm{n}$ (113 $\mathrm{mg}, 0.21$ $\mathrm{mmol}, 97 \%$ yield) as a white amorphous solid.

Analytical data for $6 \mathrm{n}:{ }^{1} \mathrm{H}$ NMR $\left(500 \mathrm{MHz}, \mathrm{CDCl}_{3}\right) \delta 7.82(\mathrm{~d}, J=7.9 \mathrm{~Hz}, 1 \mathrm{H}), 7.72(\mathrm{~d}, J=8.2 \mathrm{~Hz}, 2 \mathrm{H}), 7.68-$ $7.59(\mathrm{~m}, 2 \mathrm{H}), 7.58-7.49(\mathrm{~m}, 1 \mathrm{H}), 7.22-7.09(\mathrm{~m}, 7 \mathrm{H}), 5.00-4.89(\mathrm{~m}, 1 \mathrm{H}), 4.55(\mathrm{~d}, J=16.5 \mathrm{~Hz}, 1 \mathrm{H}), 4.20$ $(\mathrm{s}, 1 \mathrm{H}), 4.03(\mathrm{~d}, J=16.5 \mathrm{~Hz}, 1 \mathrm{H}), 3.65(\mathrm{~s}, 1 \mathrm{H}), 3.31(\mathrm{t}, J=6.5 \mathrm{~Hz}, 2 \mathrm{H}) ;{ }^{13} \mathrm{C} \mathrm{NMR}\left(126 \mathrm{MHz}, \mathrm{CDCl}_{3}\right) \delta 165.94$, $140.41,140.21$, 135.87, 133.99, 132.57, 132.04, 131.26, 130.92, 130.61, 129.37 (x2), 129.11, 128.51 (x2), 127.25, 126.89 (x2), 124.70, 124.58, 122.53, 95.24, 60.86, 43.53, 37.66; HRMS (ESI) calcd for $\left(\mathrm{C}_{25} \mathrm{H}_{21} \mathrm{~F}_{3} \mathrm{~N}_{3} \mathrm{O}{ }_{5} \mathrm{~S}\right)^{+}[\mathrm{M}+\mathrm{H}]^{+}: 532.1149$, found: 532.1149 .<smiles>C=C1CN(c2ccc(Cl)cc2)C(=O)[C@@H](Cc2ccccc2)N1[N+]#N</smiles>

(R)-3-benzyl-1-(4-chlorophenyl)-5-methylene-4-((4-nitrophenyl)sulfonyl)piperazin-2-one

(6o): Prepared according to the general method using $50(92 \mathrm{mg}, 0.2 \mathrm{mmol}), \mathrm{Pd}_{2}\left(\mathrm{dba}_{3}\right)^{\circ} \mathrm{CHCl}_{3}(3.1 \mathrm{mg}, 3.0$ $\mu \mathrm{mol})$, DPEphos (3.6 mg, $6.6 \mu \mathrm{mol})$, propargyl carbonate $2 \mathrm{a}(41 \mathrm{mg}, 0.26 \mathrm{mmol})$ in $\mathrm{CH}_{2} \mathrm{Cl}_{2}(2.0 \mathrm{~mL})$ for 28 h. Purified by flash column chromatography $\left(2: 1 \mathrm{CH}_{2} \mathrm{Cl}_{2}\right.$ :Hexanes to $1 \% \mathrm{MeOH}$ in $\left.\mathrm{CH}_{2} \mathrm{Cl}_{2}\right)$ to afford 60 (100 mg, $0.20 \mathrm{mmol}, 99 \%$ yield) as a white amorphous solid.

Analytical data for 6o: ${ }^{1} \mathrm{H}$ NMR $\left(500 \mathrm{MHz}, \mathrm{CDCl}_{3}\right) \delta 7.81$ (dd, $\left.J=7.9,1.2 \mathrm{~Hz}, 1 \mathrm{H}\right), 7.62$ (ddd, $J=9.6,7.5$, $1.4 \mathrm{~Hz}, 2 \mathrm{H}), 7.56-7.50(\mathrm{~m}, 1 \mathrm{H}), 7.41(\mathrm{~d}, J=8.5 \mathrm{~Hz}, 2 \mathrm{H}), 7.20-7.07(\mathrm{~m}, 5 \mathrm{H}), 6.98(\mathrm{~d}, J=8.5 \mathrm{~Hz}, 2 \mathrm{H}), 4.96$ $-4.88(\mathrm{~m}, 1 \mathrm{H}), 4.53(\mathrm{dd}, J=16.5,1.7 \mathrm{~Hz}, 1 \mathrm{H}), 4.18(\mathrm{~s}, 1 \mathrm{H}), 4.00(\mathrm{~d}, J=16.5 \mathrm{~Hz}, 1 \mathrm{H}), 3.73-3.63(\mathrm{~m}, 1 \mathrm{H})$, $3.30(\mathrm{dd}, J=6.5,4.4 \mathrm{~Hz}, 2 \mathrm{H}) ;{ }^{13} \mathrm{C}$ NMR $\left(126 \mathrm{MHz}, \mathrm{CDCl}_{3}\right) \delta 165.95,140.35,135.96,135.60,134.49$, 133.93, 132.63, 132.01, 131.21, 129.99, 129.78 (x2), 129.38 (x2), 128.48 (x2), 127.21, 124.57, 95.05, 60.86, 43.51, 37.66, 31.14, 22.28; HRMS (ESI) calcd for $\left(\mathrm{C}_{24} \mathrm{H}_{21} \mathrm{CIN}_{3} \mathrm{O}_{5} \mathrm{~S}\right)^{+}[\mathrm{M}+\mathrm{H}]^{+}: 498.0885$, found: 498.0883 . 


\section{General Procedure for the Pd-catalyzed Piperizine Formation with Substituted Propargyl Carbonates}

General Method: Diamine substrate 1a (1 equiv.), $\mathrm{Pd}_{2}(\mathrm{dba})_{3} \cdot \mathrm{CHCl}_{3}(0.015$ equiv., $3 \mathrm{~mol} \% \mathrm{Pd}$ ) and DPEphos ( 0.033 equiv.) were added to a oven-dried $16 \times 100$ borosilicate test tube. The test tube was covered with a rubber septum and sealed with Teflon tape. The test tube was purged with nitrogen, anhydrous $\mathrm{CH}_{2} \mathrm{Cl}_{2}(0.1 \mathrm{M})$ was added and the mixture was maintained under a positive pressure of nitrogen. In the case of a heterogeneous mixture sonication was used to break up larger particles of insoluble substrate. After 2 min the substituted propargyl carbonate 2 (1.3 equiv.) was added to the reaction mixture via syringe. The test tube was purged again with nitrogen. After consumption of starting material as judged by TLC, the reaction was concentrated and purified by flash column chromatography to give the desired products.<smiles>[As]N1CCN([As])C(=Cc2ccccc2)C1</smiles>

(Z)-2-benzylidene-1,4-ditosylpiperazine (3m): Prepared according to the general method using 1a (74 $\mathrm{mg}, 0.2 \mathrm{mmol}), \mathrm{Pd}_{2}(\mathrm{dba})_{3} \cdot \mathrm{CHCl}_{3}(3.1 \mathrm{mg}, 3.0 \mu \mathrm{mol})$, DPEphos (3.6 mg, $\left.6.6 \mu \mathrm{mol}\right)$, propargyl carbonate $\mathbf{2 b}$ (60 mg, $0.26 \mathrm{mmol})$ in $\mathrm{CH}_{2} \mathrm{Cl}_{2}(2.0 \mathrm{~mL})$ for $45 \mathrm{~min}$. Purified by flash column chromatography (8:1 to 4:1 Hexanes:EtOAc) to afford $3 \mathrm{~m}$ (43 $\mathrm{mg}, 0.09 \mathrm{mmol}, 45 \%$ yield, 10:1 mixture of olefin isomers) as a yellow solid.

Prepared according to a modification of the general method using $1 \mathrm{a}$ (37 mg, $0.1 \mathrm{mmol}), \mathrm{Pd}_{2}(\mathrm{dba})_{3} \cdot \mathrm{CHCl}_{3}$ (1.6 mg, $1.5 \mu \mathrm{mol})$, DPEphos $(1.8 \mathrm{mg}, 3.3 \mu \mathrm{mol})$, propargyl carbonate $2 \mathbf{b}(30 \mathrm{mg}, 0.13 \mathrm{mmol})$ in $\mathrm{CH}_{2} \mathrm{Cl}_{2}$ $(1.0 \mathrm{~mL})$ for $5 \mathrm{~h}$. Prior to addition of propargyl carbonate the reaction mixture was cooled to $0{ }^{\circ} \mathrm{C}$ for 30 min. Purified by flash column chromatography (8:1 to 4:1 Hexanes:EtOAc) to afford $3 \mathrm{~m} \mathrm{(24} \mathrm{mg,} 0.05$ $\mathrm{mmol}, 50 \%$ yield, $20: 1$ mixture of olefin isomers) as a yellow foam.

Analytical data for 3m: ${ }^{1} \mathrm{H}$ NMR $\left(500 \mathrm{MHz}, \mathrm{CDCl}_{3}\right) \delta 7.63(\mathrm{~d}, J=8.3 \mathrm{~Hz}, \mathrm{~min}), 7.59(\mathrm{~d}, J=$ $10.0 \mathrm{~Hz}, 2 \mathrm{H}), 7.43(\mathrm{~d}, J=8.2 \mathrm{~Hz}, \min ), 7.36(\mathrm{~d}, J=7.7 \mathrm{~Hz}, \min ), 7.28(\mathrm{~m}, 5 \mathrm{H}), 7.25-7.06(\mathrm{~m}$, $6 \mathrm{H}), 6.91(\mathrm{~d}, J=8.0 \mathrm{~Hz}, 2 \mathrm{H}), 6.74(\mathrm{~s}, \min ), 6.50(\mathrm{~s}, 1 \mathrm{H}), 4.0-3.0$ (br. s, 3H) $3.85-3.66$ (m, $1 \mathrm{H}), 3.55(\mathrm{~s}, 1 \mathrm{H}), 3.16-2.97(\mathrm{~m}, 1 \mathrm{H}), 2.43(\mathrm{~s}, 3 \mathrm{H}), 2.39(\mathrm{~s}, \mathrm{~min}), 2.26(\mathrm{~s}, 3 \mathrm{H}) ;{ }^{13} \mathrm{C}$ NMR $(126$ $\left.\mathrm{MHz}, \mathrm{CDCl}_{3}\right) \delta 143.98,143.63,136.36,133.92,132.56,131.01,129.79(\mathrm{x} 2), 129.69,129.65$, 129.21, 129.14 (x2), 128.90 (x2), 128.73, 128.63, 127.99 (x2), 127.84, 127.73 (x2), 127.57, 127.56 (x2), 127.26, 50.93, 46.95, 46.28, 44.44, 44.13, 21.52, 21.36; HRMS (ESI) calcd for $\left(\mathrm{C}_{25} \mathrm{H}_{27} \mathrm{~N}_{2} \mathrm{O}_{4} \mathrm{~S}_{2}\right)^{+}[\mathrm{M}+\mathrm{H}]^{+}:$483.1407, found: 483.1405 . 
<smiles>[3H]N1CCN([AsH3-])C(=C)C1c1ccccc1</smiles>

2-methylene-3-phenyl-1,4-ditosylpiperazine (3n): Prepared according to the general method using 1a (74 mg, $0.2 \mathrm{mmol}$ ), $\mathrm{Pd}_{2}\left(\mathrm{dba}_{3} \cdot \mathrm{CHCl}_{3}(3.1 \mathrm{mg}, 3.0 \mu \mathrm{mol}\right.$ ), DPEphos (3.6 mg, $6.6 \mu \mathrm{mol}$ ), propargyl carbonate 2b $(60 \mathrm{mg}, 0.26 \mathrm{mmol})$ in $\mathrm{CH}_{2} \mathrm{Cl}_{2}(2.0 \mathrm{~mL})$ for $45 \mathrm{~min}$. Purified by flash column chromatography (8:1 to 4:1 Hexanes:EtOAc) to afford $3 \mathrm{n}(43 \mathrm{mg}, 0.09 \mathrm{mmol}, 45 \%$ yield) as a white amorphous solid.

Analytical data for $3 \mathrm{n}:{ }^{1} \mathrm{H}$ NMR $\left(500 \mathrm{MHz}, \mathrm{CDCl}_{3}\right) \delta 7.69(\mathrm{~d}, J=8.2 \mathrm{~Hz}, 2 \mathrm{H}), 7.39(\mathrm{~d}, J=8.2 \mathrm{~Hz}, 2 \mathrm{H}), 7.29(\mathrm{~d}$, $J=7.9 \mathrm{~Hz}, 2 \mathrm{H}), 7.16(\mathrm{~m}, 7 \mathrm{H}), 5.40(\mathrm{~s}, 1 \mathrm{H}), 5.31(\mathrm{~s}, 1 \mathrm{H}), 4.72(\mathrm{~s}, 1 \mathrm{H}), 3.57(\mathrm{~m}, 1 \mathrm{H}), 3.48(\mathrm{~m}, 2 \mathrm{H}), 3.39(\mathrm{~m}$, 1H), $2.43(\mathrm{~s}, 3 \mathrm{H}), 2.41(\mathrm{~s}, 3 \mathrm{H}) ;{ }^{13} \mathrm{C}$ NMR $\left(126 \mathrm{MHz}, \mathrm{CDCl}_{3}\right) \delta 143.96,137.54,137.33,129.80(\mathrm{x} 2), 129.48$ (x2), 128.53 (x3), 127.46 (x2), 127.45 (x2), 127.41 (x2), 126.26 (x2), 105.61 (x2), 62.77 (x2), 44.06, 42.96, 21.53; HRMS (ESI) calcd for $\left(\mathrm{C}_{25} \mathrm{H}_{27} \mathrm{~N}_{2} \mathrm{O}_{4} \mathrm{~S}_{2}\right)^{+}[\mathrm{M}+\mathrm{H}]^{+}:$483.1407, found: 483.1411.

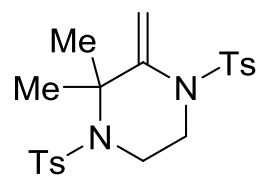

2,2-dimethyl-3-methylene-1,4-ditosylpiperazine (30): Prepared according to the general method using 1a (74 mg, $0.2 \mathrm{mmol}), \mathrm{Pd}_{2}(\mathrm{dba})_{3} \cdot \mathrm{CHCl}_{3}(3.1 \mathrm{mg}, 3.0 \mu \mathrm{mol})$, DPEphos (3.6 mg, $6.6 \mu \mathrm{mol}$ ), propargyl carbonate $2 \mathrm{~d}$ ( $48 \mathrm{mg}, 0.26 \mathrm{mmol})$ in $\mathrm{CH}_{2} \mathrm{Cl}_{2}(2.0 \mathrm{~mL}$ ) for $3 \mathrm{~h}$. Purified by flash column chromatography (8:1 to 4:1 Hexanes:EtOAc) to afford $30(52 \mathrm{mg}, 0.12 \mathrm{mmol}, 60 \%$ yield) as a white amorphous solid.

Analytical data for 3o: ${ }^{1} \mathrm{H}$ NMR $\left(500 \mathrm{MHz}, \mathrm{CDCl}_{3}\right) \delta 7.69-7.60(\mathrm{~m}, 4 \mathrm{H}), 7.27(\mathrm{dd}, J=4.3,3.8 \mathrm{~Hz}, 4 \mathrm{H})$, $5.30(\mathrm{~d}, J=1.5 \mathrm{~Hz}, 1 \mathrm{H}), 5.03(\mathrm{~d}, J=1.5 \mathrm{~Hz}, 1 \mathrm{H}), 3.62-3.57(\mathrm{~m}, 2 \mathrm{H}), 3.54(\mathrm{t}, J=5.1 \mathrm{~Hz}, 2 \mathrm{H}), 2.43(\mathrm{~d}, J=$ $2.3 \mathrm{~Hz}, 6 \mathrm{H}), 1.43(\mathrm{~s}, 6 \mathrm{H}) ;{ }^{13} \mathrm{C}$ NMR $\left(126 \mathrm{MHz} \mathrm{CDCl}_{3}\right) \delta 145.96,143.98,143.96,143.16,129.60$ (x2), 129.55 (x2), 127.56 (x2), 126.85 (x3), 107.86, 65.25, 47.58, 42.40, 26.63 (x2), 21.50, 21.44; HRMS (ESI) calcd for $\left(\mathrm{C}_{21} \mathrm{H}_{27} \mathrm{~N}_{2} \mathrm{O}_{4} \mathrm{~S}_{2}\right)^{+}[\mathrm{M}+\mathrm{H}]^{+}: 435.1407$, found: 435.1411 .<smiles>C/C=C1/C(C)N([125I])CCN1[13CH3]</smiles>

(Z)-2-ethylidene-3-methyl-1,4-ditosylpiperazine (3p): Prepared according to the general method using 1a (55 mg, $0.15 \mathrm{mmol}), \mathrm{Pd}_{2}(\mathrm{dba})_{3} \cdot \mathrm{CHCl}_{3}(2.4 \mathrm{mg}, 2.3 \mu \mathrm{mol})$, DPEphos (2.7 mg, $\left.5.0 \mu \mathrm{mol}\right)$, propargyl carbonate $2 \mathrm{e}(37 \mathrm{mg}, 0.20 \mathrm{mmol})$ in $\mathrm{CH}_{2} \mathrm{Cl}_{2}(1.5 \mathrm{~mL})$ for $24 \mathrm{~h}$. Purified by flash column chromatography (8:1 to 4:1 Hexanes:EtOAc) to afford $3 p$ ( $37 \mathrm{mg}, 0.09 \mathrm{mmol}, 57 \%$ yield) as a white solid.

Analytical data for $3 p$ : (mp: $\left.157^{\circ} \mathrm{C}\right),{ }^{1} \mathrm{H}$ NMR $\left(500 \mathrm{MHz}, \mathrm{CDCl}_{3}\right) \delta 7.75(\mathrm{~d}, J=8.3 \mathrm{~Hz}, 2 \mathrm{H}), 7.64(\mathrm{~d}, J=8.2$ $\mathrm{Hz}, 2 \mathrm{H}), 7.36-7.29(\mathrm{~m}, 2 \mathrm{H}), 7.28-7.18(\mathrm{~m}, 2 \mathrm{H}), 5.60(\mathrm{~d}, J=6.9 \mathrm{~Hz}, 1 \mathrm{H}), 4.51(\mathrm{~d}, J=6.9 \mathrm{~Hz}, 1 \mathrm{H}), 3.58(\mathrm{dt}$, $J=13.4,2.9 \mathrm{~Hz}, 1 \mathrm{H}), 3.47(\mathrm{dt}, J=13.6,3.0 \mathrm{~Hz}, 1 \mathrm{H}), 3.42-3.33(\mathrm{~m}, 1 \mathrm{H}), 3.02-2.94(\mathrm{~m}, 1 \mathrm{H}), 2.43(\mathrm{~s}, 3 \mathrm{H})$, 
$2.41(\mathrm{~s}, 3 \mathrm{H}), 1.60(\mathrm{~d}, J=6.9 \mathrm{~Hz}, 3 \mathrm{H}), 1.21(\mathrm{~d}, J=6.9 \mathrm{~Hz}, 3 \mathrm{H}) ;{ }^{13} \mathrm{C} \mathrm{NMR}\left(126 \mathrm{MHz}, \mathrm{CDCl}_{3}\right) \delta 143.86,143.44$, 137.31, 137.05, 133.79, 129.68 (x2), 129.67 (x2), 127.52 (x2), 127.15 (x2), 125.48, 55.29, 46.56, 39.44, 21.49, 21.46, 17.91, 13.97; HRMS (ESI) calcd for $\left(\mathrm{C}_{21} \mathrm{H}_{27} \mathrm{~N}_{2} \mathrm{O}_{4} \mathrm{~S}_{2}\right)^{+}[\mathrm{M}+\mathrm{H}]^{+}: 483.1407$, found: 483.1411.<smiles>CC(C)=C1C(c2ccccc2)N([AsH3])CCN1[AsH3-]</smiles>

2-phenyl-3-(propan-2-ylidene)-1,4-ditosylpiperazine (3q): Prepared according to the general method using 1a (37 mg, $0.1 \mathrm{mmol}), \mathrm{Pd}_{2}\left(\mathrm{dba}_{3} \cdot \mathrm{CHCl}_{3}(1.6 \mathrm{mg}, 1.5 \mu \mathrm{mol})\right.$, DPEphos (1.8 mg, $\left.3.3 \mu \mathrm{mol}\right)$, propargyl carbonate $2 \mathrm{f}(34 \mathrm{mg}, 0.13 \mathrm{mmol})$ in $\mathrm{CH}_{2} \mathrm{Cl}_{2}(1.0 \mathrm{~mL})$ for $24 \mathrm{~h}$ at $40{ }^{\circ} \mathrm{C}$. Purified by flash column chromatography (8:1 to 3:1 Hexanes:EtOAc) to afford $3 q$ (10 mg, $0.02 \mathrm{mmol}, 20 \%$ yield) as a clear oil.

Analytical data for 3q: ${ }^{1} \mathrm{H}$ NMR $\left(500 \mathrm{MHz}, \mathrm{CDCl}_{3}\right) \delta 7.75(\mathrm{~d}, J=8.2 \mathrm{~Hz}, 2 \mathrm{H}), 7.70(\mathrm{~d}, J=8.3 \mathrm{~Hz}, 2 \mathrm{H}), 7.31-$ $7.25(\mathrm{~m}, 5 \mathrm{H}), 7.23(\mathrm{~d}, J=8.1 \mathrm{~Hz}, 2 \mathrm{H}), 7.16(\mathrm{dd}, J=8.2,1.4 \mathrm{~Hz}, 2 \mathrm{H}), 5.16(\mathrm{t}, J=5.7 \mathrm{~Hz}, 1 \mathrm{H}), 3.64(\mathrm{t}, J=6.6$ $\mathrm{Hz}, 2 \mathrm{H}), 3.33(\mathrm{q}, J=6.4 \mathrm{~Hz}, 2 \mathrm{H}), 2.42(\mathrm{~s}, 3 \mathrm{H}), 2.35(\mathrm{~s}, 3 \mathrm{H}), 1.65(\mathrm{~s}, 6 \mathrm{H}) ;{ }^{13} \mathrm{C} \mathrm{NMR}\left(126 \mathrm{MHz}, \mathrm{CDCl}_{3}\right) \delta$ 143.50, 143.31, 131.58 (x3), 129.72 (x2), 129.58 (x2), 128.56 (x2), 128.21 (x2), 127.52 (x2), 127.22 (x2), $121.98,90.57,84.20,57.31,47.98,44.52,30.70$ (x2), 21.49, 21.41; HRMS (ESI) calcd for $\left(\mathrm{C}_{27} \mathrm{H}_{31} \mathrm{~N}_{2} \mathrm{O}_{4} \mathrm{~S}_{2}\right)^{+}$ $[\mathrm{M}+\mathrm{H}]^{+}:$511.1720, found: 511.1709 .

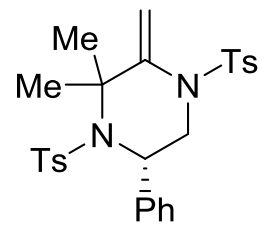

(S)-2-phenyl-5-methylene-2,2-dimethyl-1,4-ditosylpiperazine (3r): Prepared according to the general method using 1e (89 mg, $0.2 \mathrm{mmol}), \mathrm{Pd}_{2}\left(\mathrm{dba}_{3}\right) \mathrm{CHCl}_{3}(3.1 \mathrm{mg}, 3.0 \mu \mathrm{mol})$, DPEphos (3.6 mg, $\left.3.3 \mu \mathrm{mol}\right)$, propargyl carbonate $2 \mathrm{~d}(48 \mathrm{mg}, 0.26 \mathrm{mmol})$ in $\mathrm{CH}_{2} \mathrm{Cl}_{2}(2.0 \mathrm{~mL})$ for $21 \mathrm{~h}$ at $23{ }^{\circ} \mathrm{C}$. Purified by flash column chromatography (8:1 to $5: 1$ Hexanes:EtOAc) to afford $3 r$ (90 mg, $0.18 \mathrm{mmol}, 88 \%$ yield) as an off white solid.

Analytical data for $3 \mathrm{r}:\left(\mathrm{mp}: 210^{\circ} \mathrm{C}\right),{ }^{1} \mathrm{H}$ NMR $\left(500 \mathrm{MHz}, \mathrm{CDCl}_{3}\right) \delta 7.65(\mathrm{~d}, J=8.1 \mathrm{~Hz}, 2 \mathrm{H}), 7.55(\mathrm{~d}, J=8.1$ $\mathrm{Hz}, 2 \mathrm{H}), 7.35-7.29(\mathrm{~m}, 2 \mathrm{H}), 7.27-7.18(\mathrm{~m}, 7 \mathrm{H}), 5.26(\mathrm{~s}, 1 \mathrm{H}), 4.91(\mathrm{~d}, J=0.7 \mathrm{~Hz}, 1 \mathrm{H}), 4.65(\mathrm{~d}, J=0.7 \mathrm{~Hz}$, $1 \mathrm{H}), 4.39(\mathrm{dd}, J=11.6,1.4 \mathrm{~Hz}, 1 \mathrm{H}), 3.59(\mathrm{dd}, J=11.7,3.7 \mathrm{~Hz}, 1 \mathrm{H}), 2.44(\mathrm{~s}, 3 \mathrm{H}), 2.42(\mathrm{~s}, 3 \mathrm{H}), 1.62(\mathrm{~s}, 3 \mathrm{H})$, $1.21(\mathrm{~s}, 3 \mathrm{H}) ;{ }^{13} \mathrm{C}$ NMR $\left(126 \mathrm{MHz} \mathrm{CDCl}_{3}\right) \delta 144.26,143.95,143.35,138.88,137.83,129.57$ (x2), 129.46 (x2), 128.02 (x3), 127.49 (x2), 127.47 (x2), 127.34 (x2), 126.93 (x2), 105.91, 64.61, 55.67, 47.17, 28.49, 27.78, 21.53; HRMS (ESI) calcd for $\left(\mathrm{C}_{27} \mathrm{H}_{30} \mathrm{~N}_{2} \mathrm{O}_{4} \mathrm{~S}_{2} \mathrm{Na}\right)^{+}[\mathrm{M}+\mathrm{Na}]^{+}: 533.1539$, found: 533.1532 . 


\section{General Procedure for the Removal of Nosyl Group}

General Method: Based on the protocol by Floreancig and coworkers. ${ }^{27} 60$ (1.0 equiv.) and $\mathrm{K}_{2} \mathrm{CO}_{3}(3$ equiv.) are added to a dry borosilicate test tube which is then covered with a rubber septa and purged with nitrogen. Anhydrous $\mathrm{MeCN}(0.11 \mathrm{M})$ is then added via syringe and the suspension is set stirring at a high rate to prevent $\mathrm{K}_{2} \mathrm{CO}_{3}$ from settling. To this $\mathrm{PhSH}$ ( 3 equiv.) is added by syringe all at once, within five minutes a color change is noted and SM is consumed as monitored by TLC. The reaction mixture is diluted with $\mathrm{CH}_{2} \mathrm{Cl}_{2}$ and washed with water. The aqueous layer is extracted 2 times with $\mathrm{CH}_{2} \mathrm{Cl}_{2}$, the combined organic layers are then washed with brine, dried over $\mathrm{MgSO}_{4}$ and concentrated in vaccuo. The residue is then purified by flash column chromatography to afford the desired compounds.<smiles>C=C1CN(c2ccc(Cl)cc2)C(=O)[C@@H](Cc2ccccc2)N1</smiles>

(R)-3-benzyl-1-(4-chlorophenyl)-5-methylenepiperazin-2-one (7): Prepared according to the general method using 60 (50 mg, $0.1 \mathrm{mmol}), \mathrm{K}_{2} \mathrm{CO}_{3}(42 \mathrm{mg}, 0.3 \mathrm{mmol}), \mathrm{PhSH}(0.03 \mathrm{~mL}, 0.3 \mathrm{mmol})$ in $\mathrm{MeCN}(1 \mathrm{~mL})$ for 5 min. Purified by flash column chromatography $\left(2: 1 \mathrm{CH}_{2} \mathrm{Cl}_{2}:\right.$ Hexanes to $1 \% \mathrm{MeOH}$ in $\left.\mathrm{CH}_{2} \mathrm{Cl}_{2}\right)$ to afford 7 (47 mg, $0.1 \mathrm{mmol}, 99 \%$ yield) as a white amorphous solid.

Analytical data for $7:{ }^{1} \mathrm{H} N M R\left(500 \mathrm{MHz}, \mathrm{CDCl}_{3}\right) \delta 7.44(\mathrm{~d}, J=8.6 \mathrm{~Hz}, 2 \mathrm{H}), 7.37-7.31(\mathrm{~m}, 2 \mathrm{H}), 7.30-7.24$ $(\mathrm{m}, 3 \mathrm{H}), 7.06(\mathrm{~d}, J=8.6 \mathrm{~Hz}, 2 \mathrm{H}), 4.06(\mathrm{~s}, 1 \mathrm{H}), 3.88(\mathrm{dd}, J=8.3,4.0 \mathrm{~Hz}, 1 \mathrm{H}), 3.74(\mathrm{~d}, J=14.7 \mathrm{~Hz}, 1 \mathrm{H}), 3.66$ (d, $J=14.7 \mathrm{~Hz}, 1 \mathrm{H}$ ), $3.62(\mathrm{~s}, 1 \mathrm{H}), 3.37(\mathrm{dd}, J=13.8,3.9 \mathrm{~Hz}, 1 \mathrm{H}), 3.15$ (dd, $J=13.8,8.3 \mathrm{~Hz}, 1 \mathrm{H}$ ) 1.74 (br. s, $1 \mathrm{H}) ;{ }^{13} \mathrm{C}$ NMR $\left(126 \mathrm{MHz}, \mathrm{CDCl}_{3}\right) \delta 169.24,144.62,137.46,136.36,134.00,130.07(\times 2), 129.90(\times 2)$, $129.58(x 2), 128.72(x 2), 126.96,92.07,61.03,47.40,37.61$; HRMS (ESI) calcd for $\left(\mathrm{C}_{18} \mathrm{H}_{18} \mathrm{ClN}_{2} \mathrm{O}\right)^{+}[\mathrm{M}+\mathrm{H}]^{+}$: 313.1102, found: 313.1105 .

${ }^{27}$ Jung, H. H.; Floreancig, P. E. J. Org. Chem. 2007, 72, 7359. 


\section{Selected NMR Spectra}

TDM_6_171_1.5 苦

$\overbrace{\mathrm{Me}}^{\mathrm{Me}}{ }^{2 \mathrm{e}}$

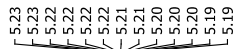

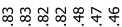

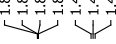

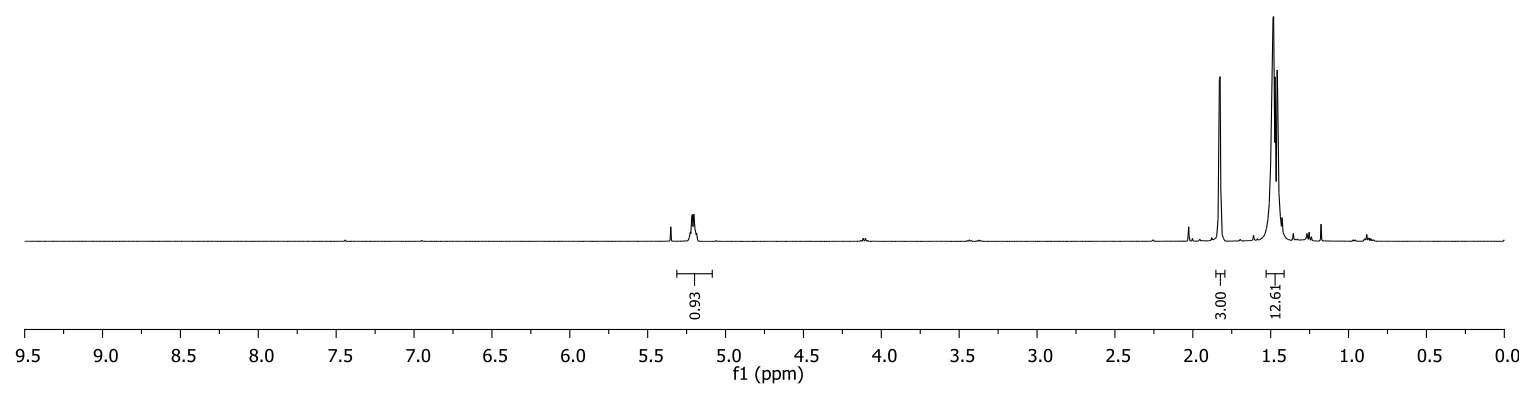

TDM_6_171_1C

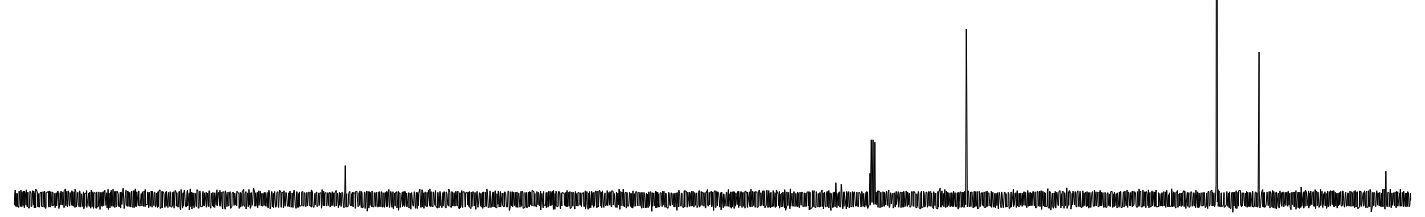

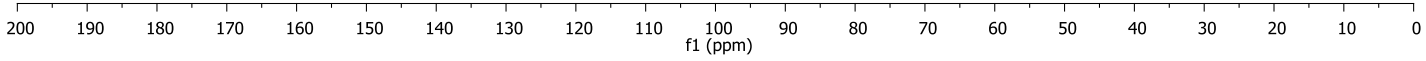




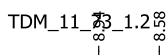

Ts $\mathrm{N}_{\mathrm{H}}^{\mathrm{Hh}}$ 1d

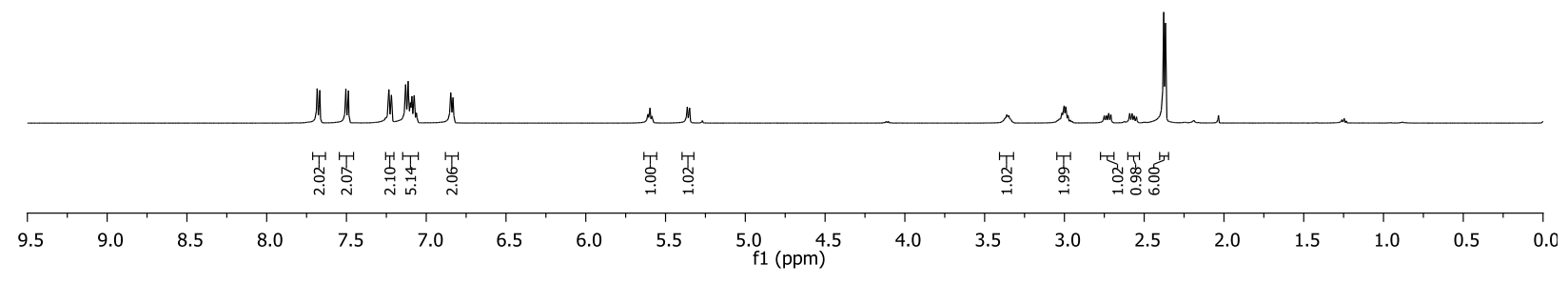

TDM_11_73_1.2C

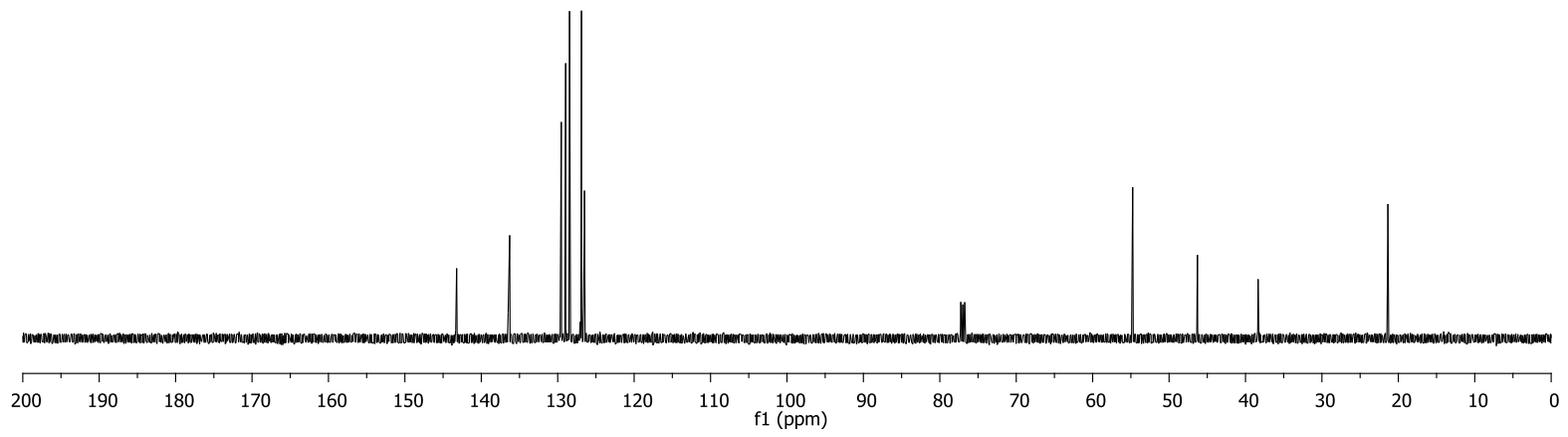


TDM_12_3_1.2 2

पर्यराँ

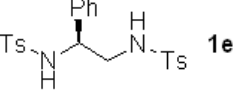

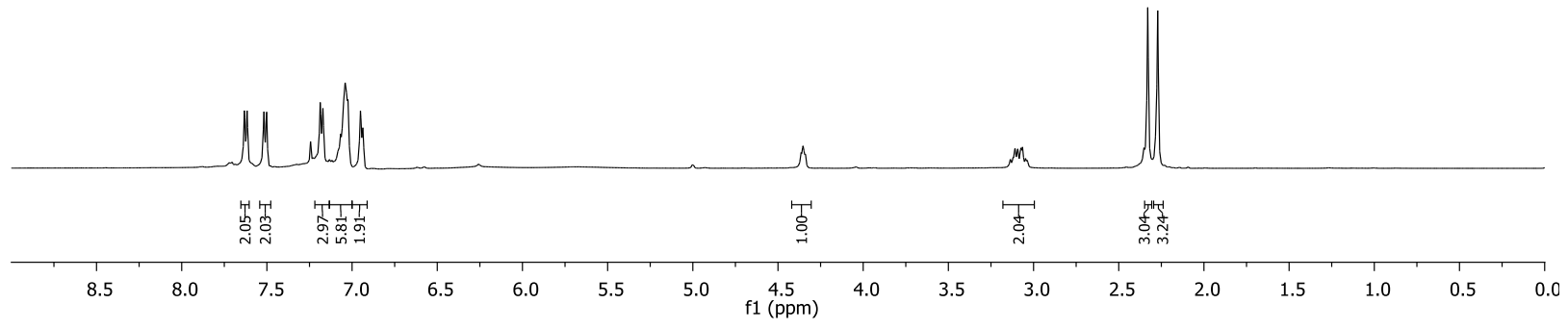

TDM_12_3_1.2C
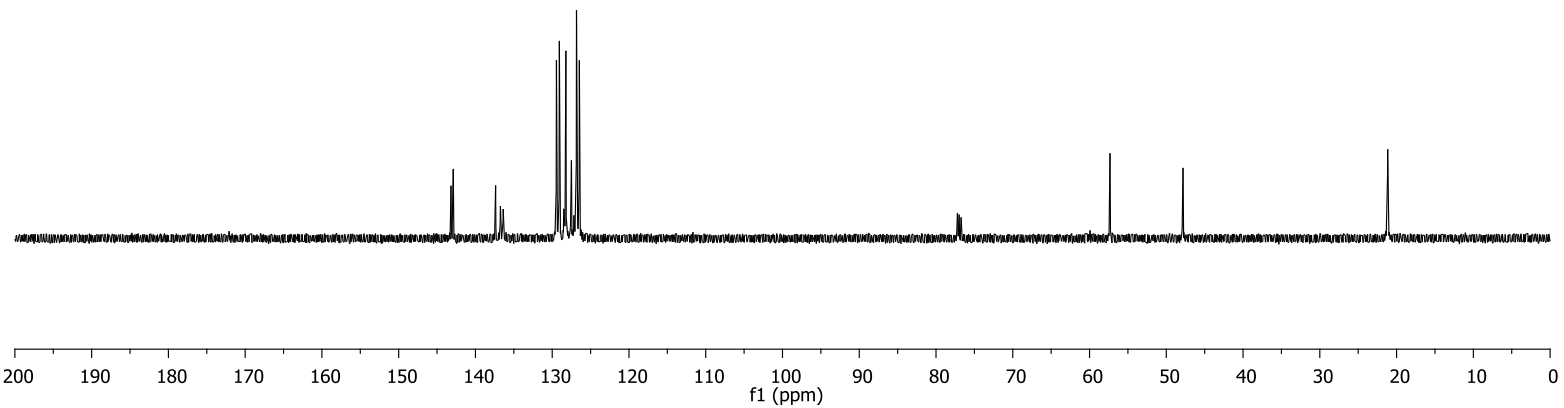


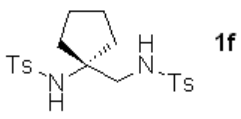

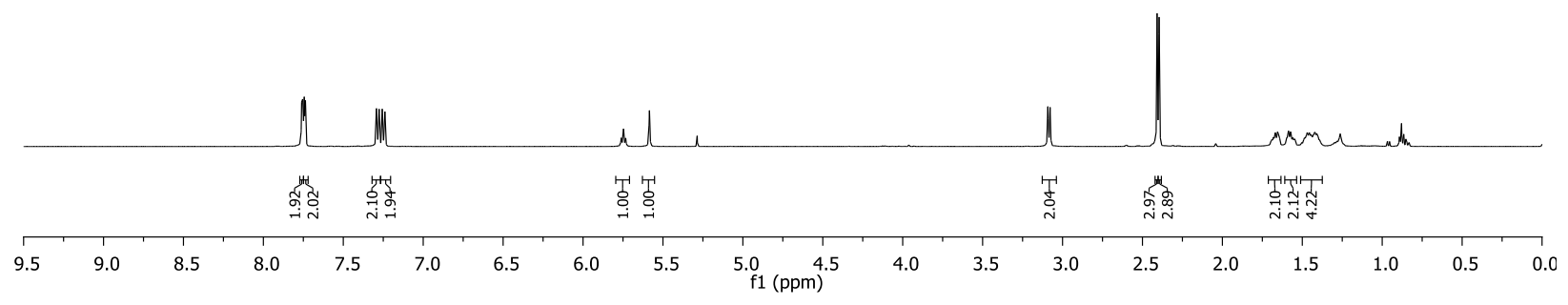

TDM_11_125_1.5C

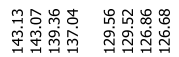

Vิ

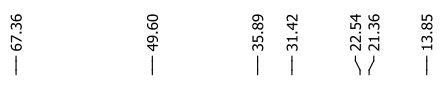

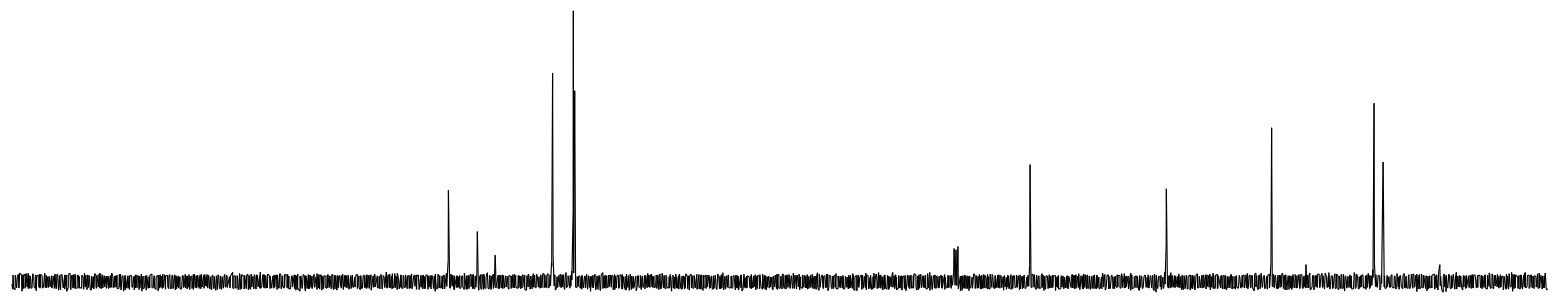




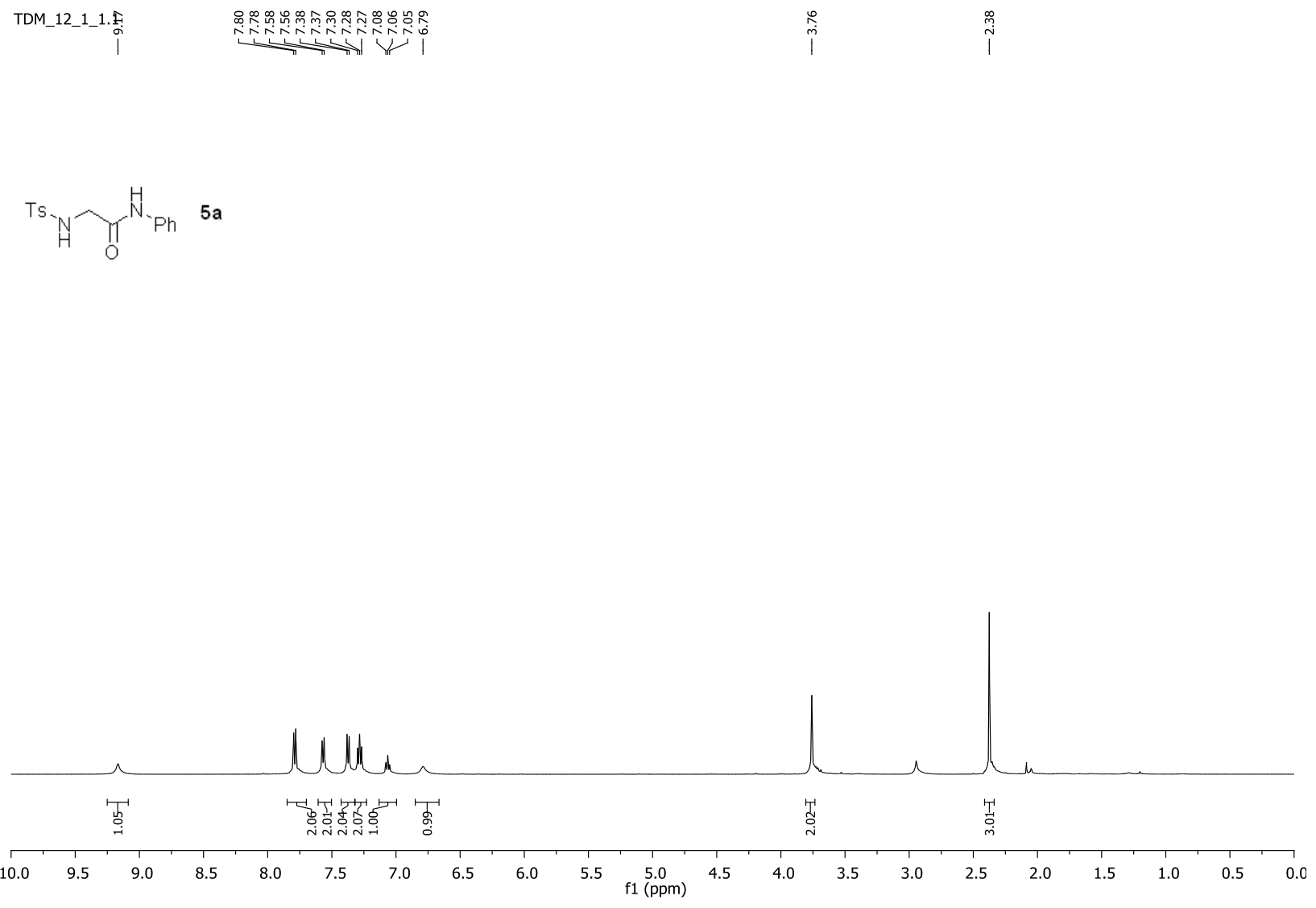

TDM_12_1_1.1C $\quad$ a
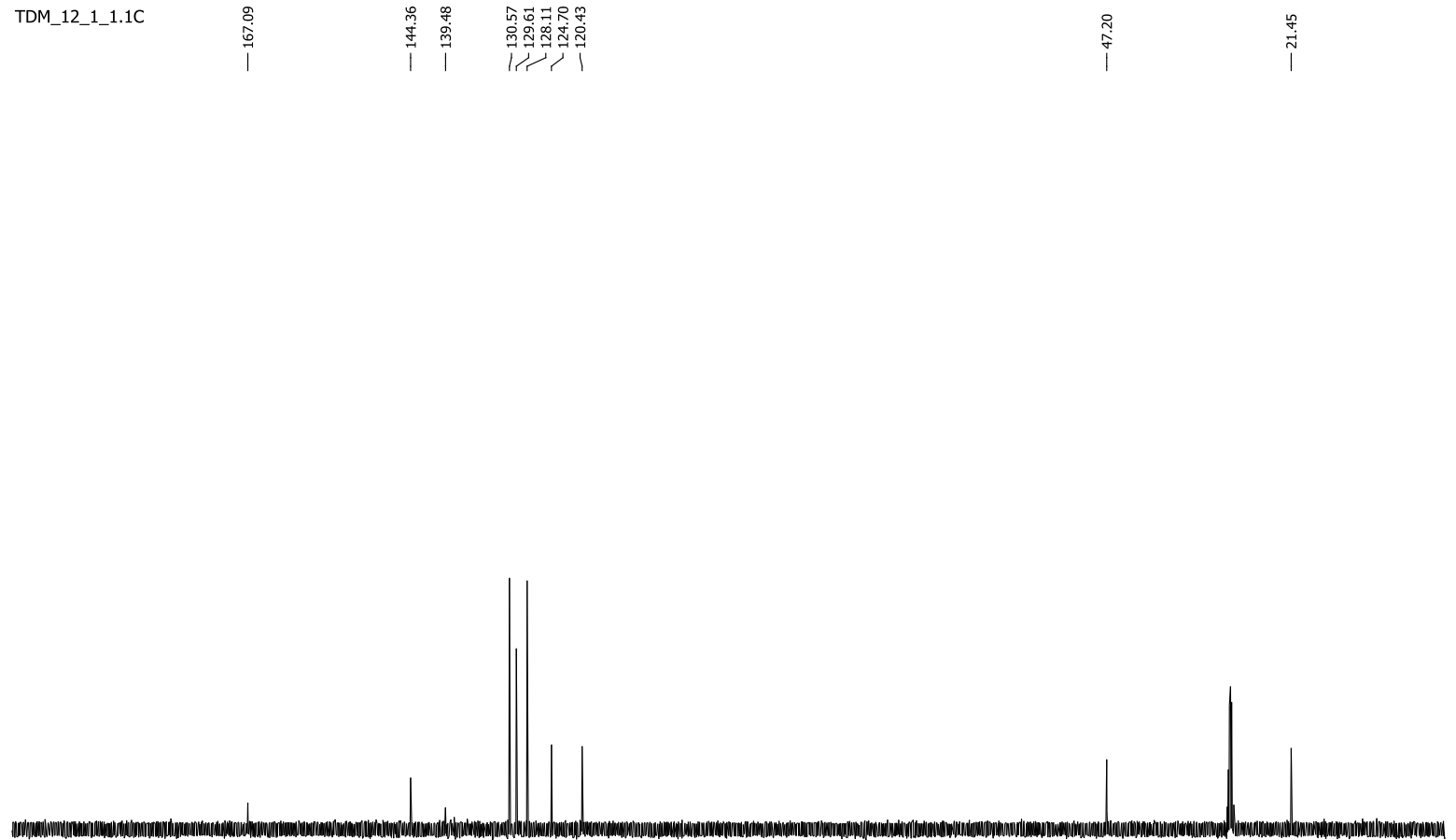

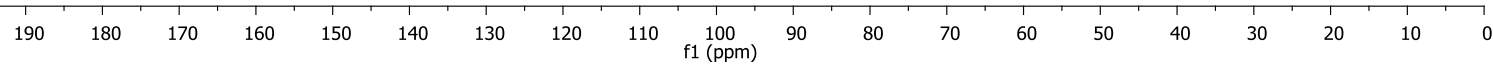




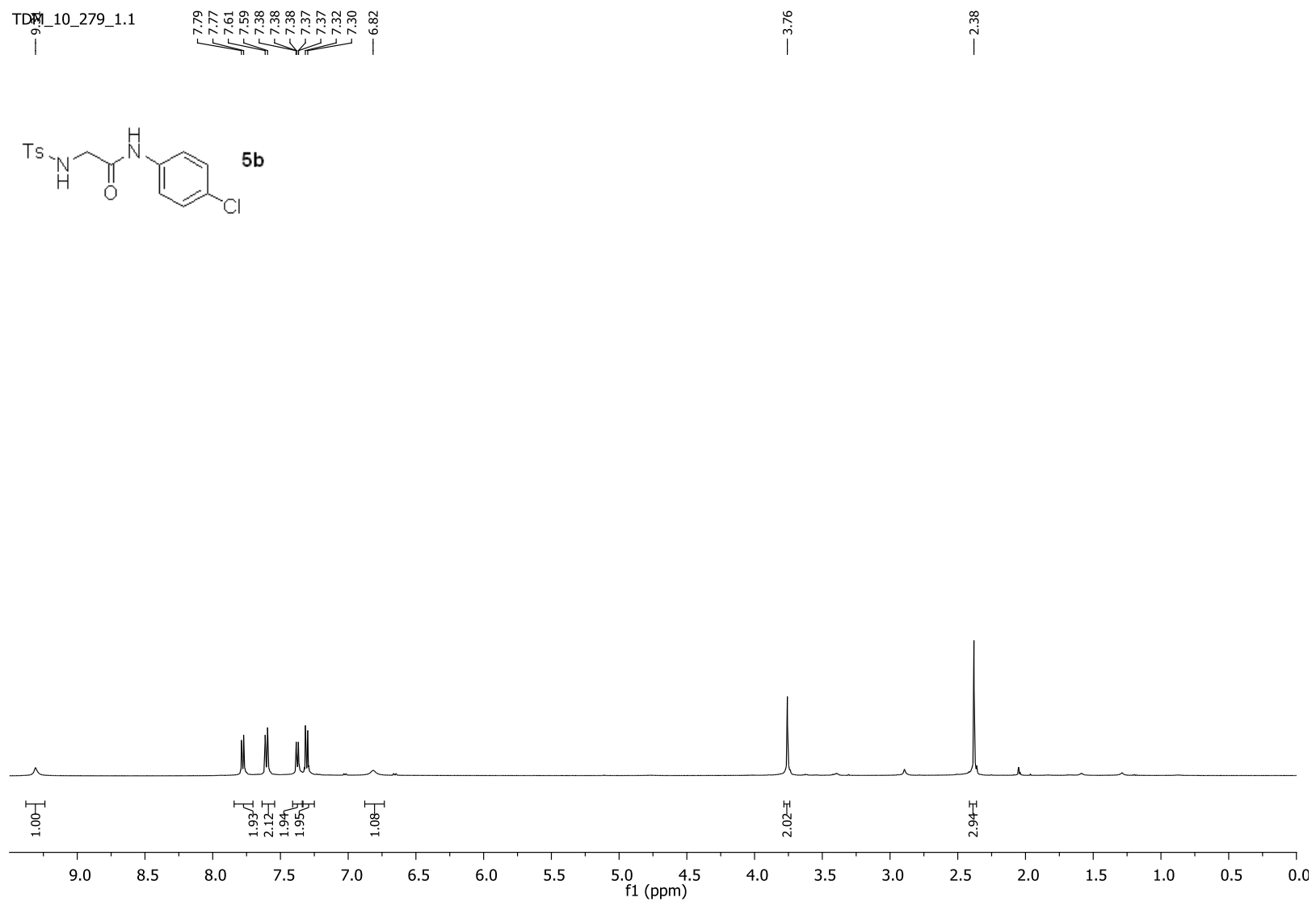

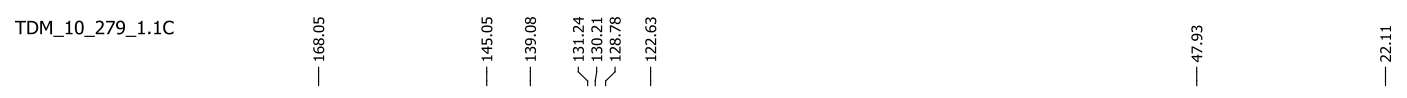

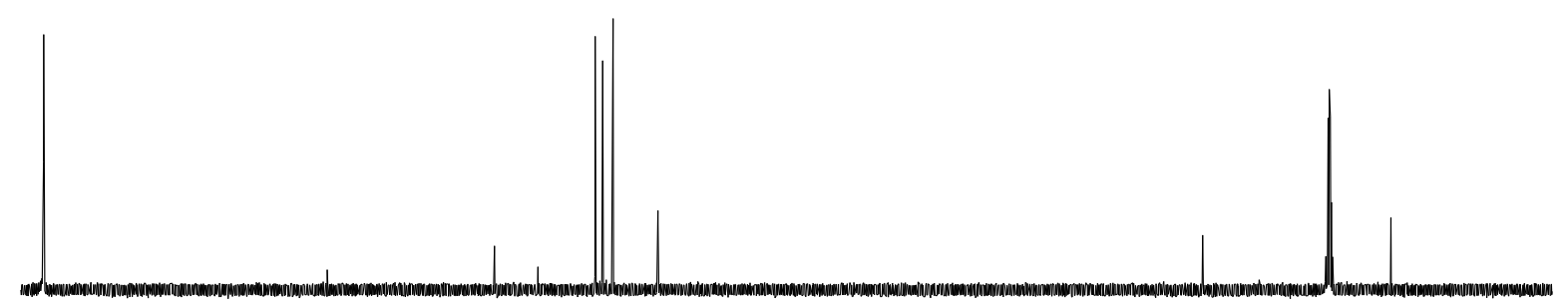

\begin{tabular}{|c|c|c|c|c|c|c|c|c|c|c|c|c|c|c|c|c|c|c|c|c|}
\hline 210 & 200 & 190 & 180 & 170 & 160 & 150 & 140 & 130 & 120 & $\begin{array}{c}110 \\
\text { f1 }\end{array}$ & $\begin{array}{c}100 \\
100)\end{array}$ & 90 & 80 & 70 & 60 & 50 & 40 & 30 & 20 & 10 \\
\hline
\end{tabular}


TDM_11_23_1. F

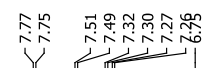

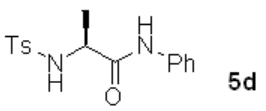
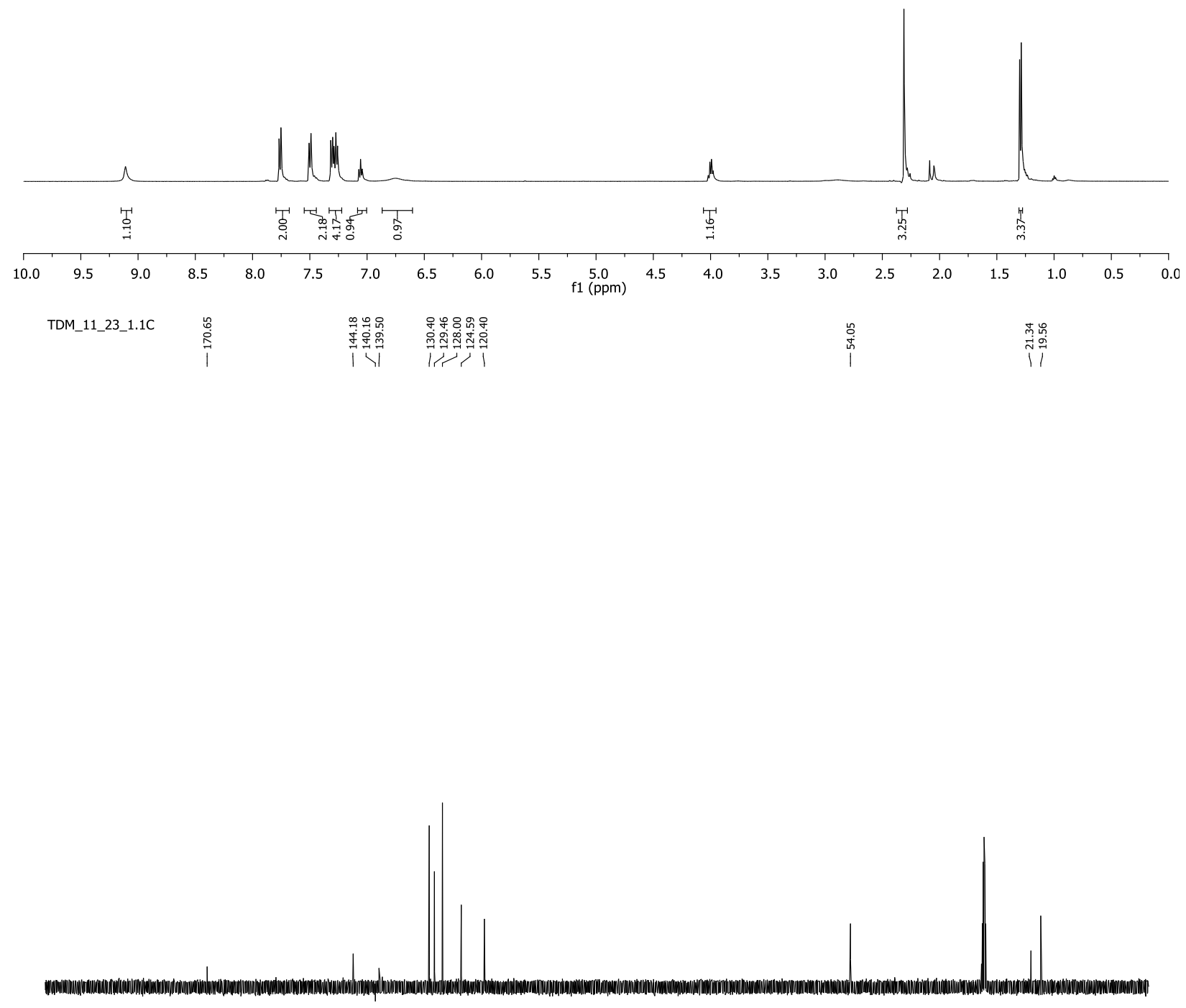

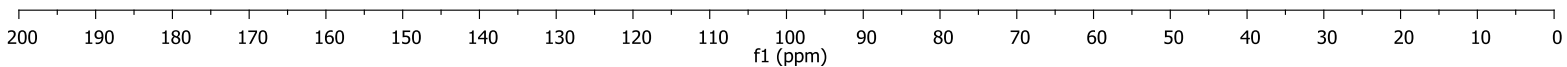


TDM_8_25_3.di

พิ่

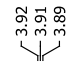

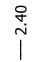

ํㅟㄲ

${ }_{\mathrm{H}}^{\text {Ts }} \prod_{\mathrm{Cl}}^{\mathrm{N}}$

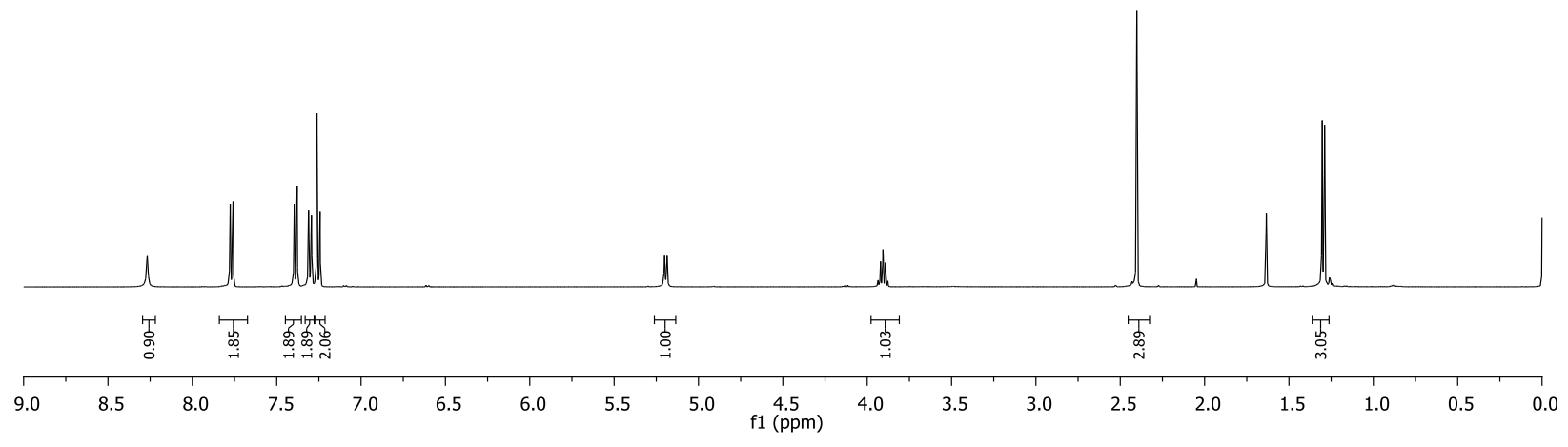

TDM_8_25_3.1C

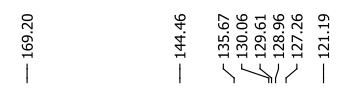
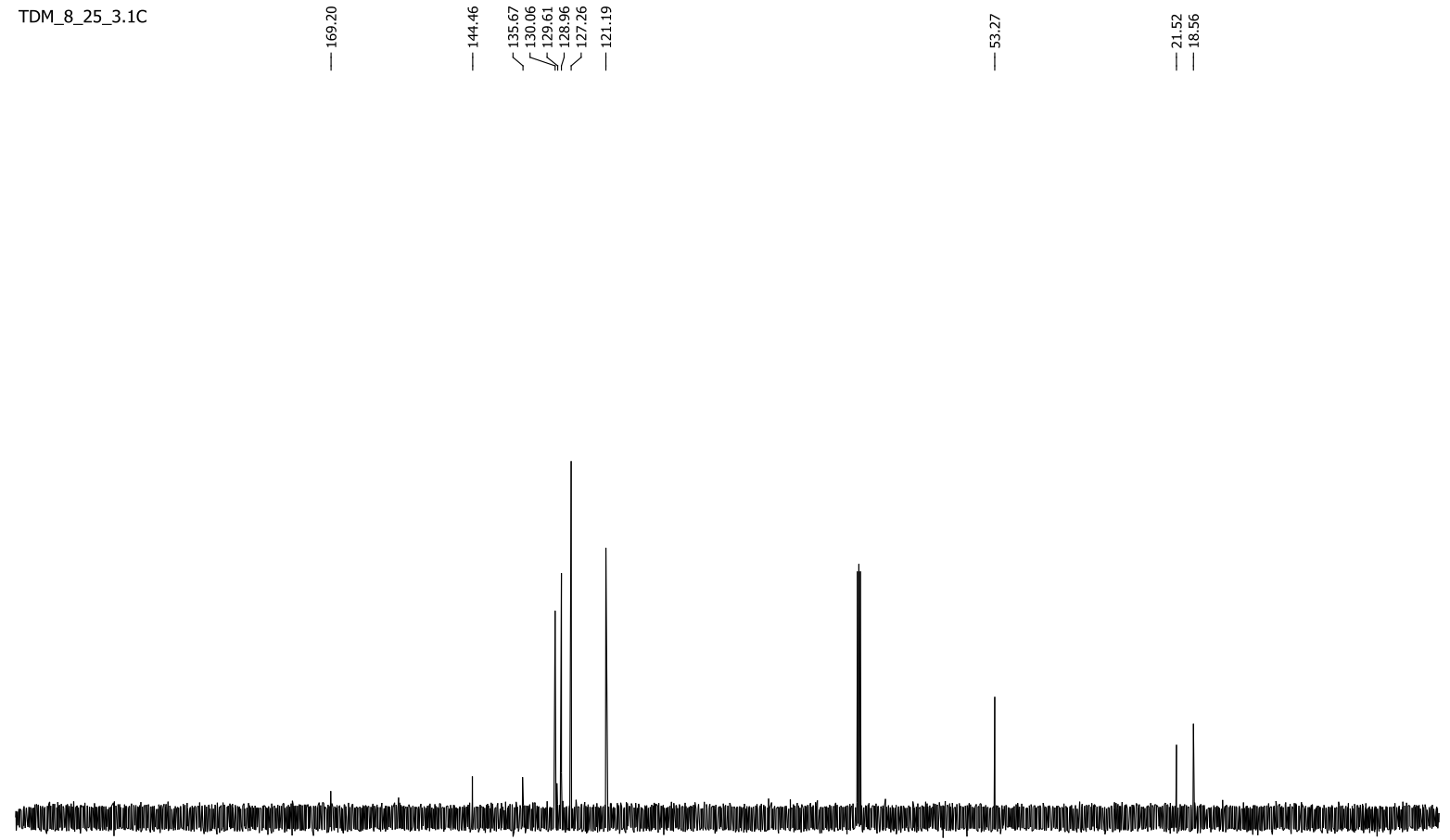

$\begin{array}{lllllllllllllllllllllllllll}220 & 210 & 200 & 190 & 180 & 170 & 160 & 150 & 140 & 130 & 120 & 110 & 100 & 90 & 80 & 70 & 60 & 50 & 40 & 30 & 20 & 10 & 0 & -10 & -20\end{array}$ 

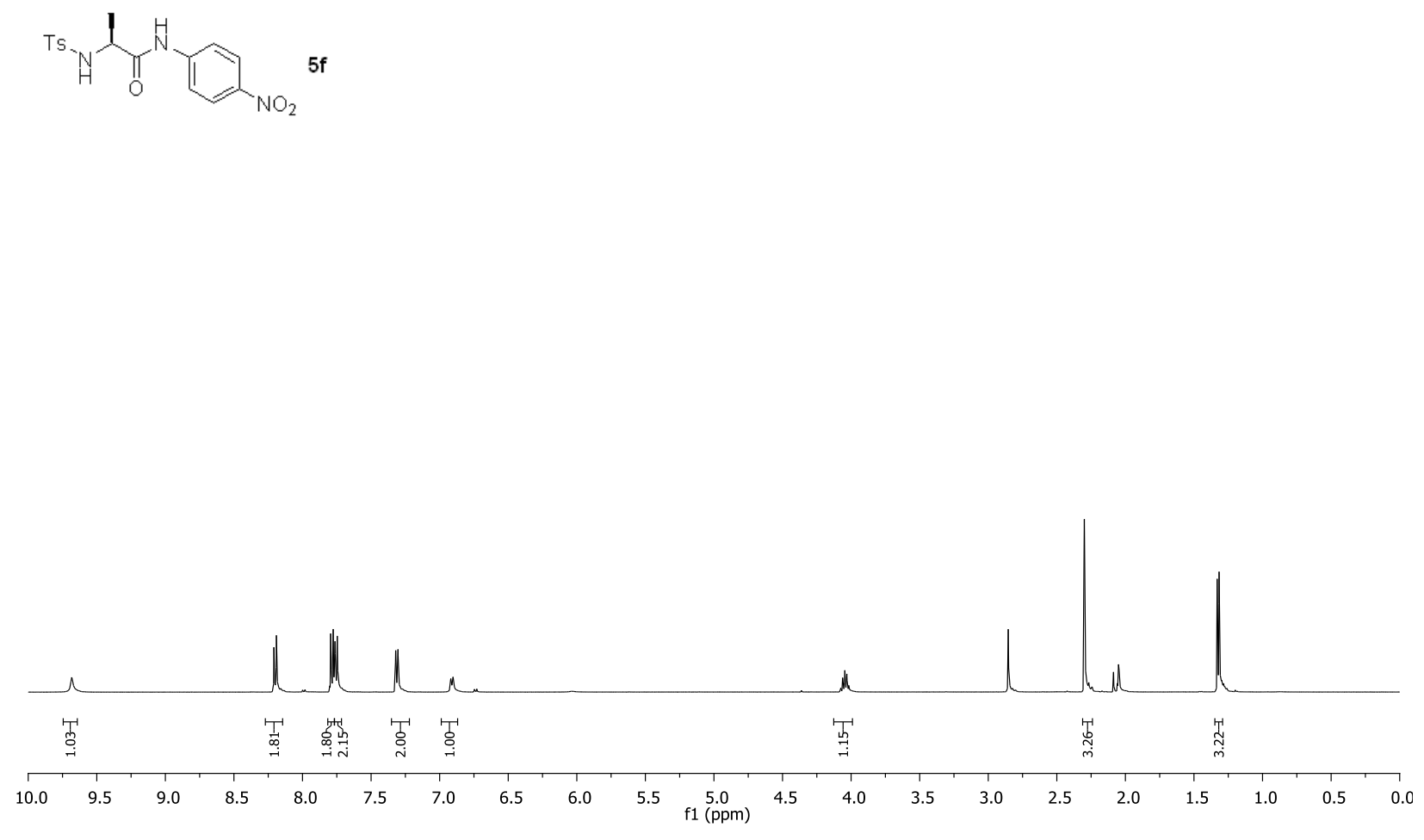

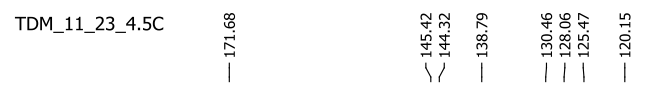
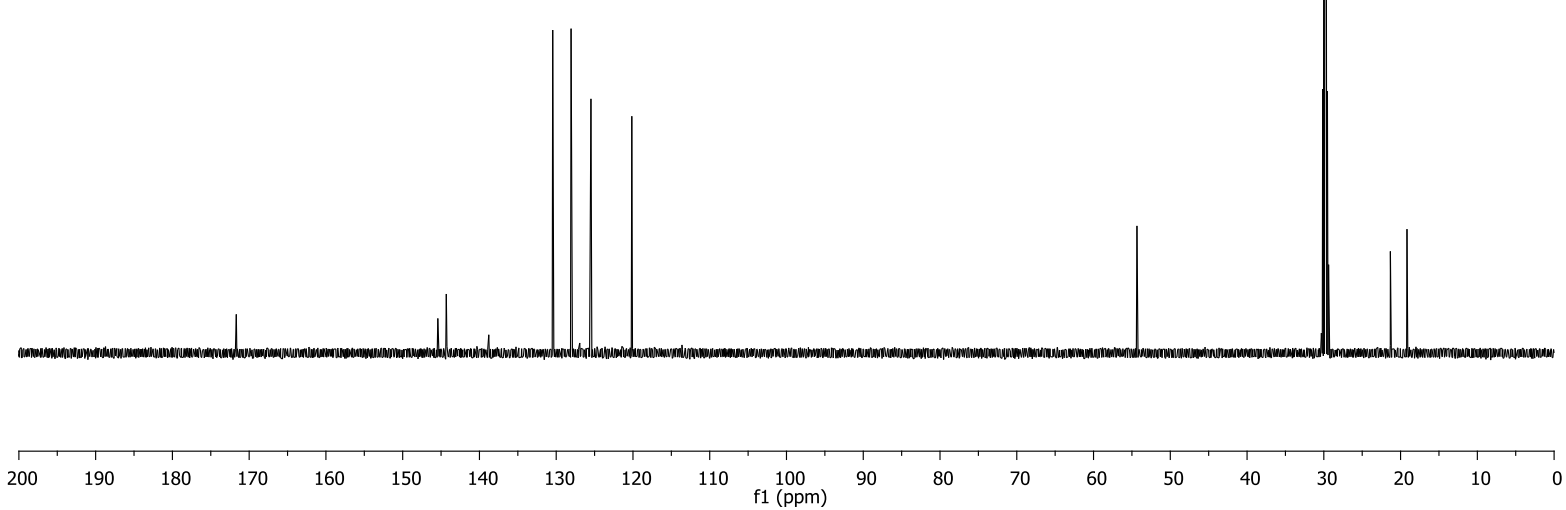


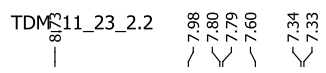

ฟูก

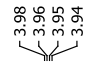

$\underset{i}{\stackrel{7}{i}}$

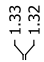

ig

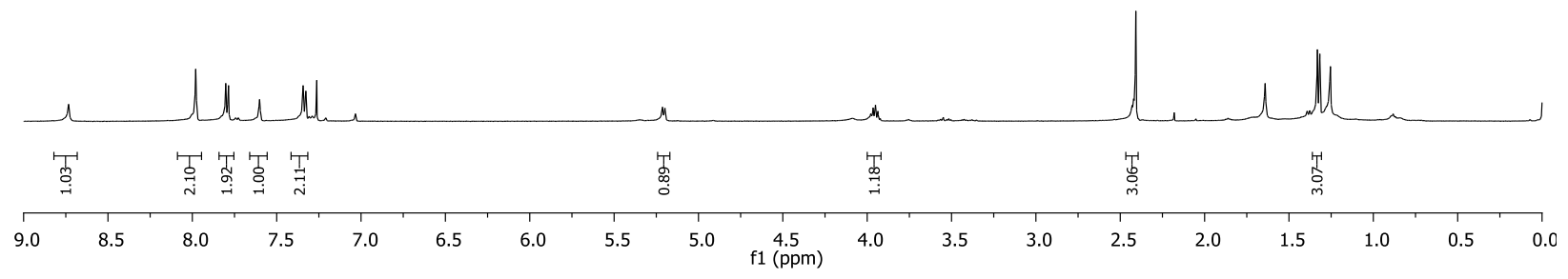

TDM_11_23_2.2C 角
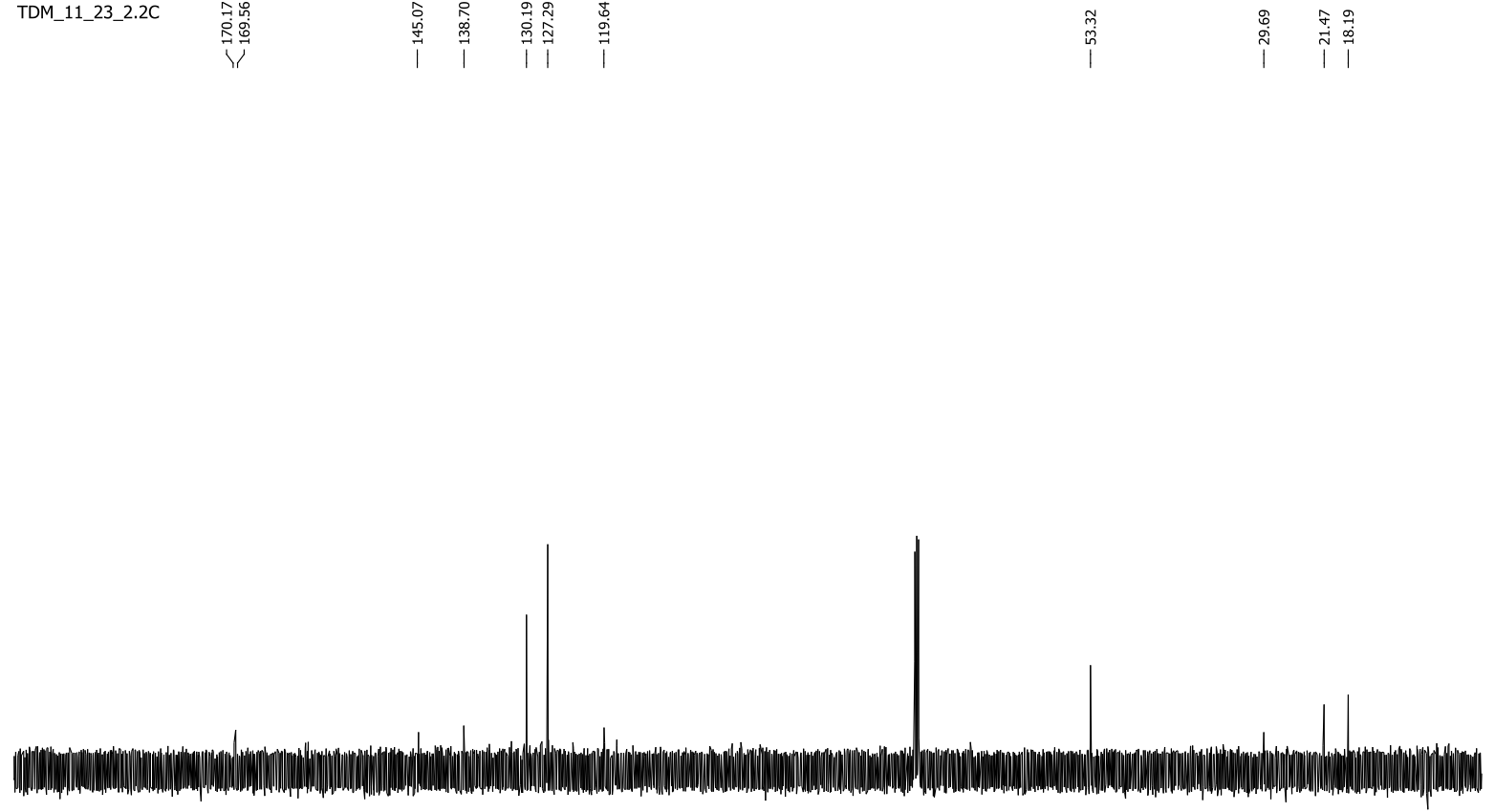

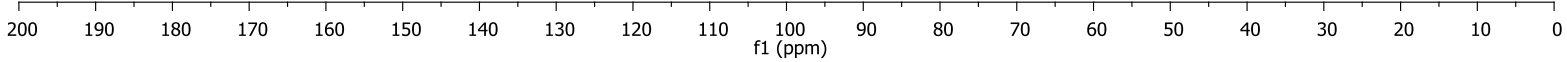


TDM_11_23_3.1
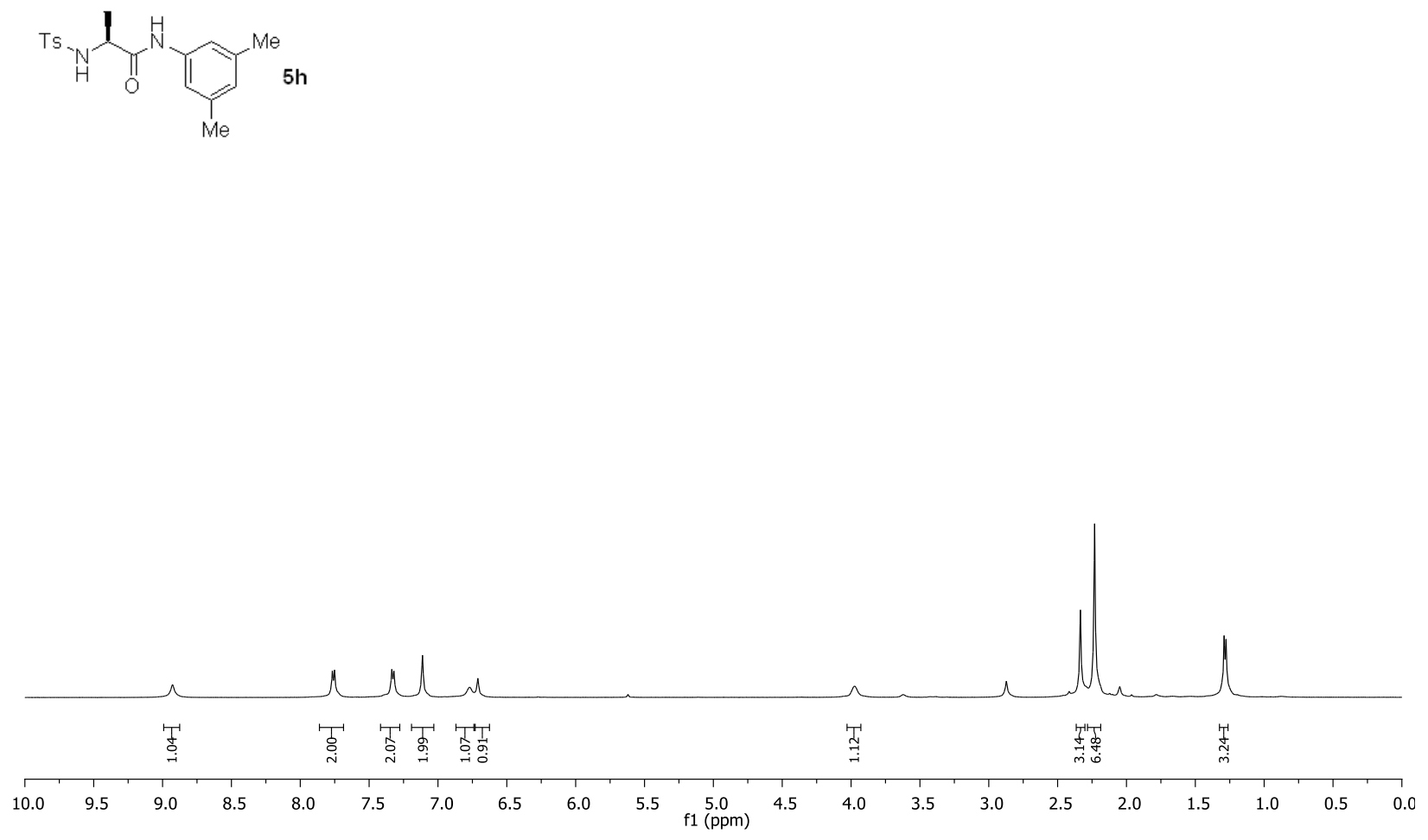

TDM_11_23_3.1C

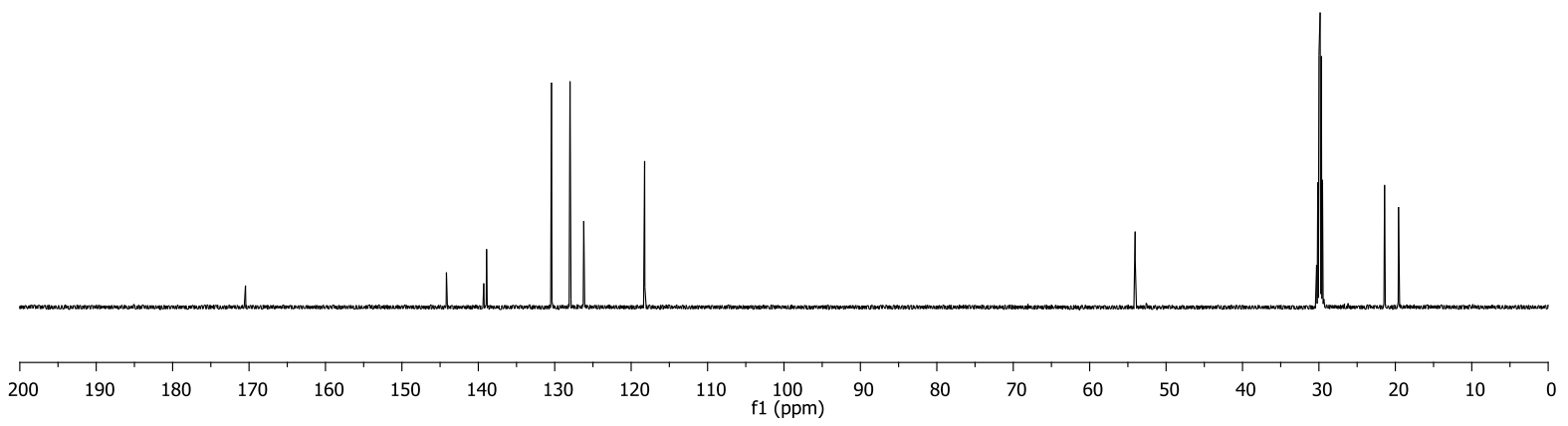


TDM_8_25_2.1
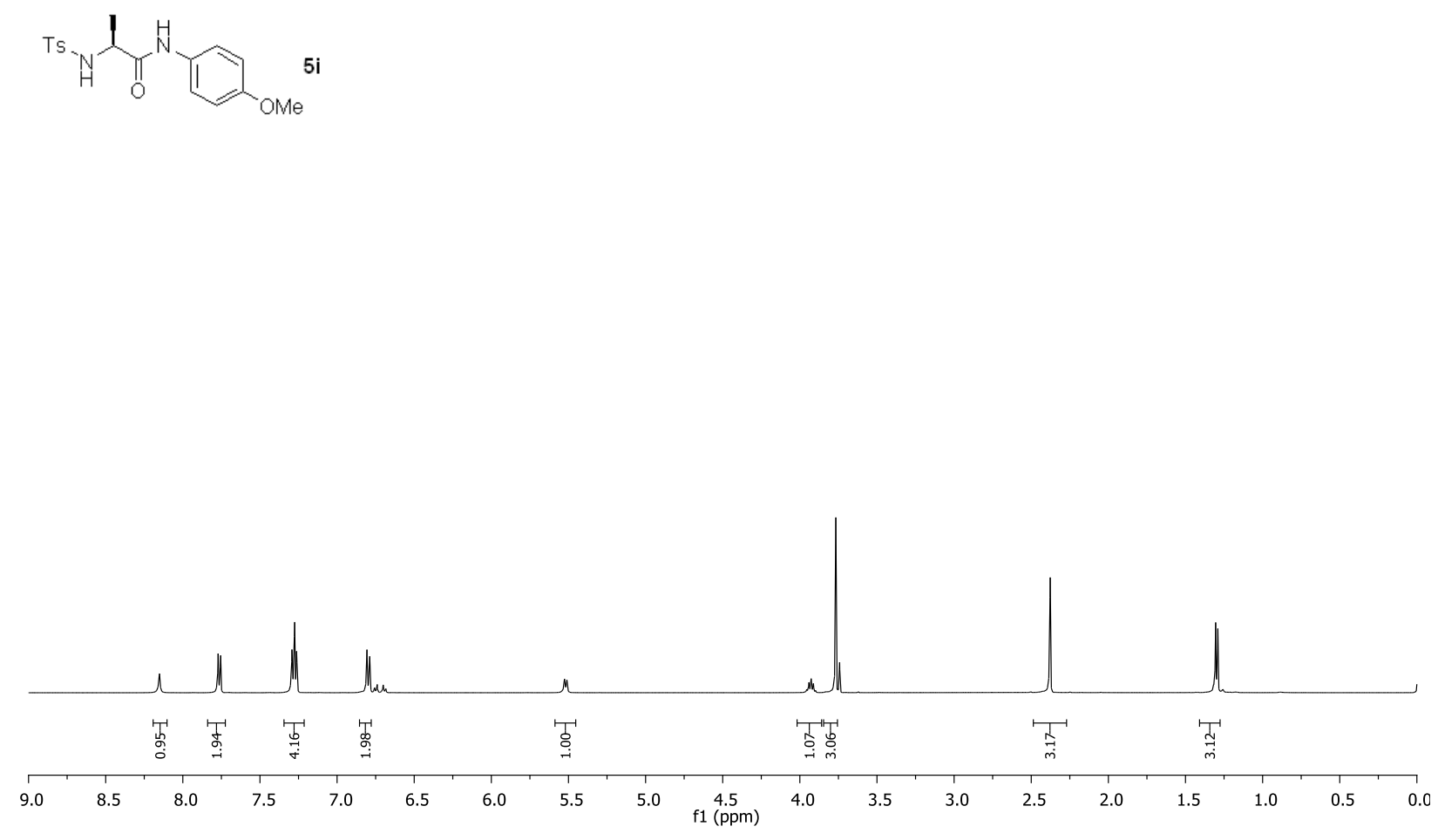

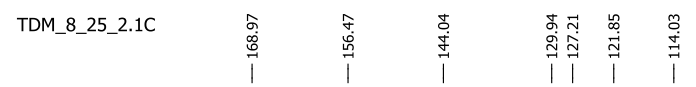

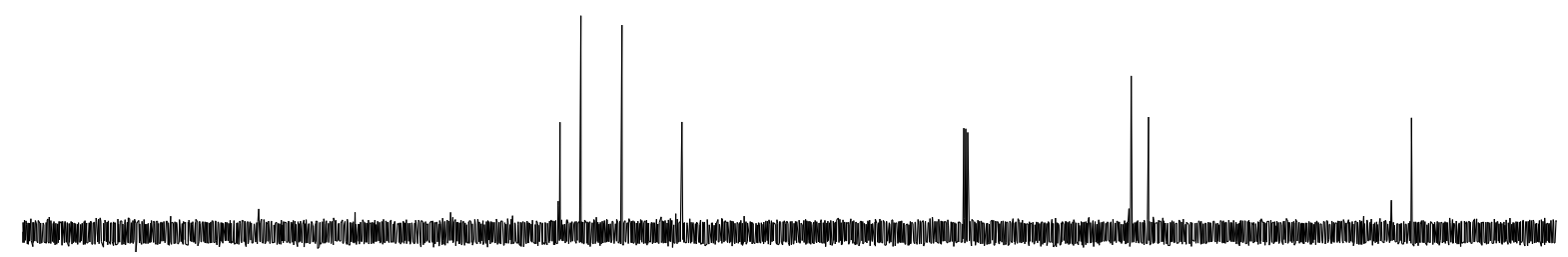

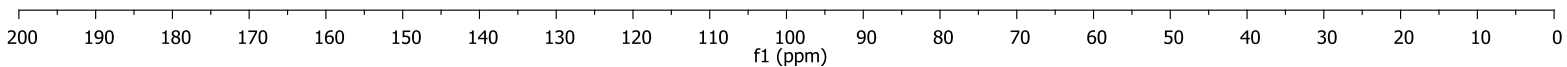


TDM_8_69_1.4

i

$\mathrm{C}_{\mathrm{O}}^{\mathrm{N}}$

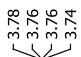

$\stackrel{\substack{\pi \\ i}}{\mid}$

我

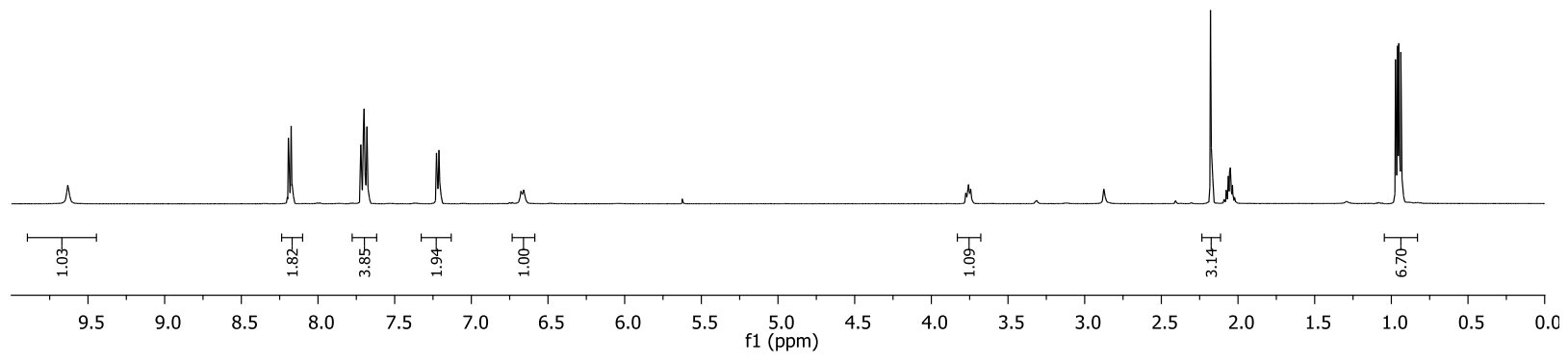

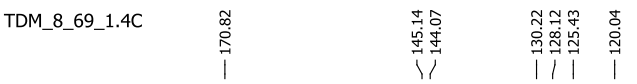

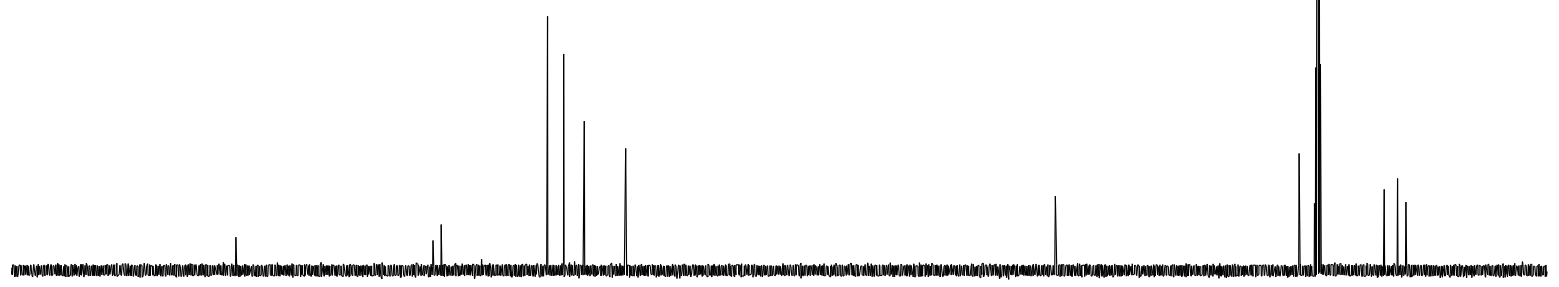
$190 \quad 180$ 170 160 150 140 130 120 110 100
$f 1(\mathrm{ppm})$ $90 \quad 80$ $70 \quad 60$ 50 
$\overbrace{\mathrm{O}}^{\mathrm{H}} \overbrace{\mathrm{H}}^{\mathrm{H}} \mathbf{5 k}$
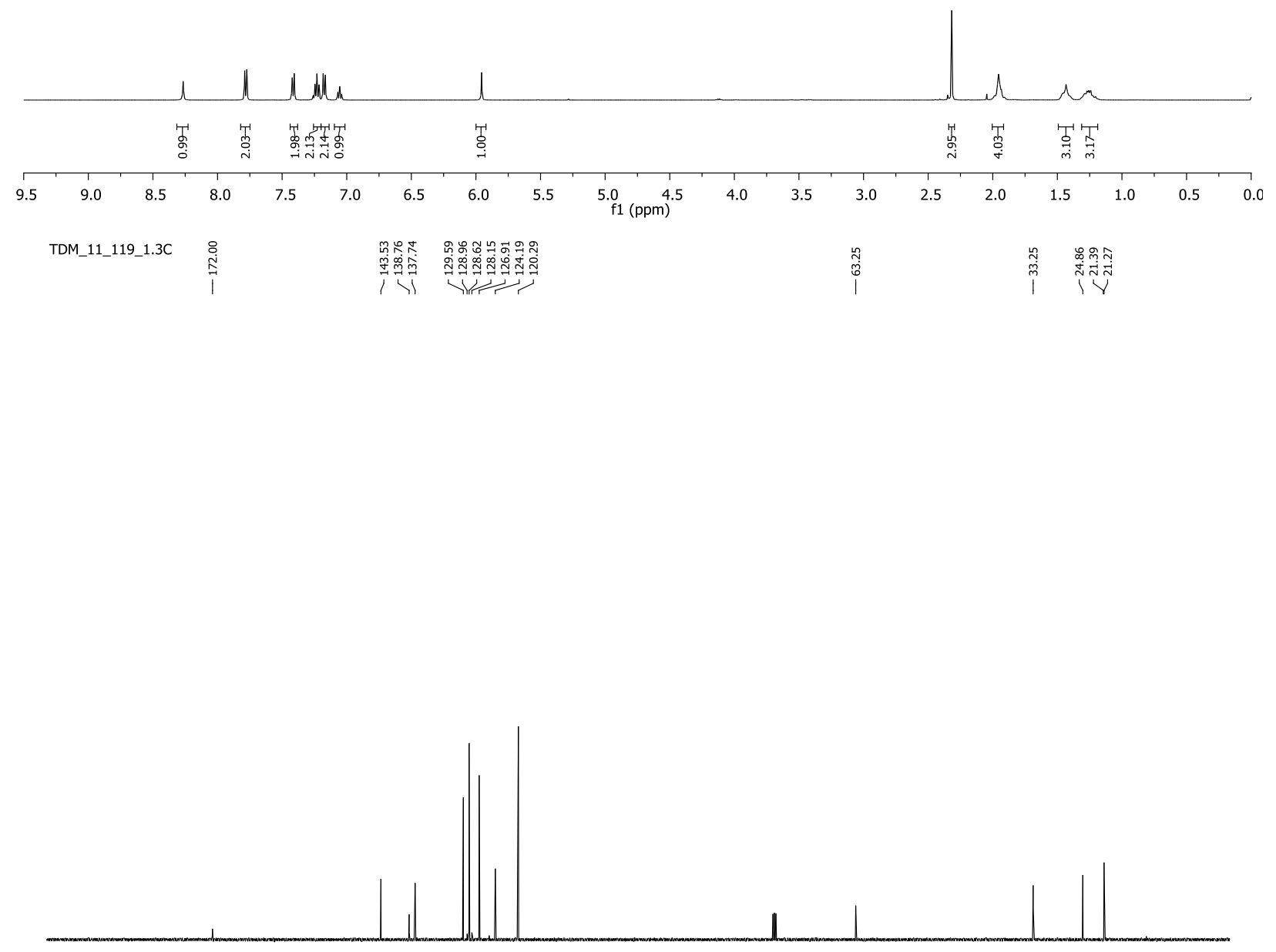

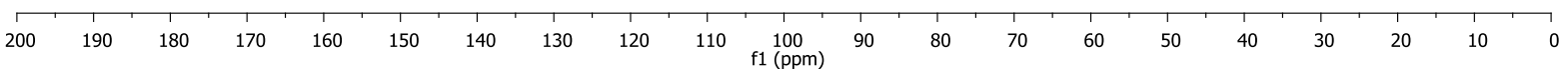



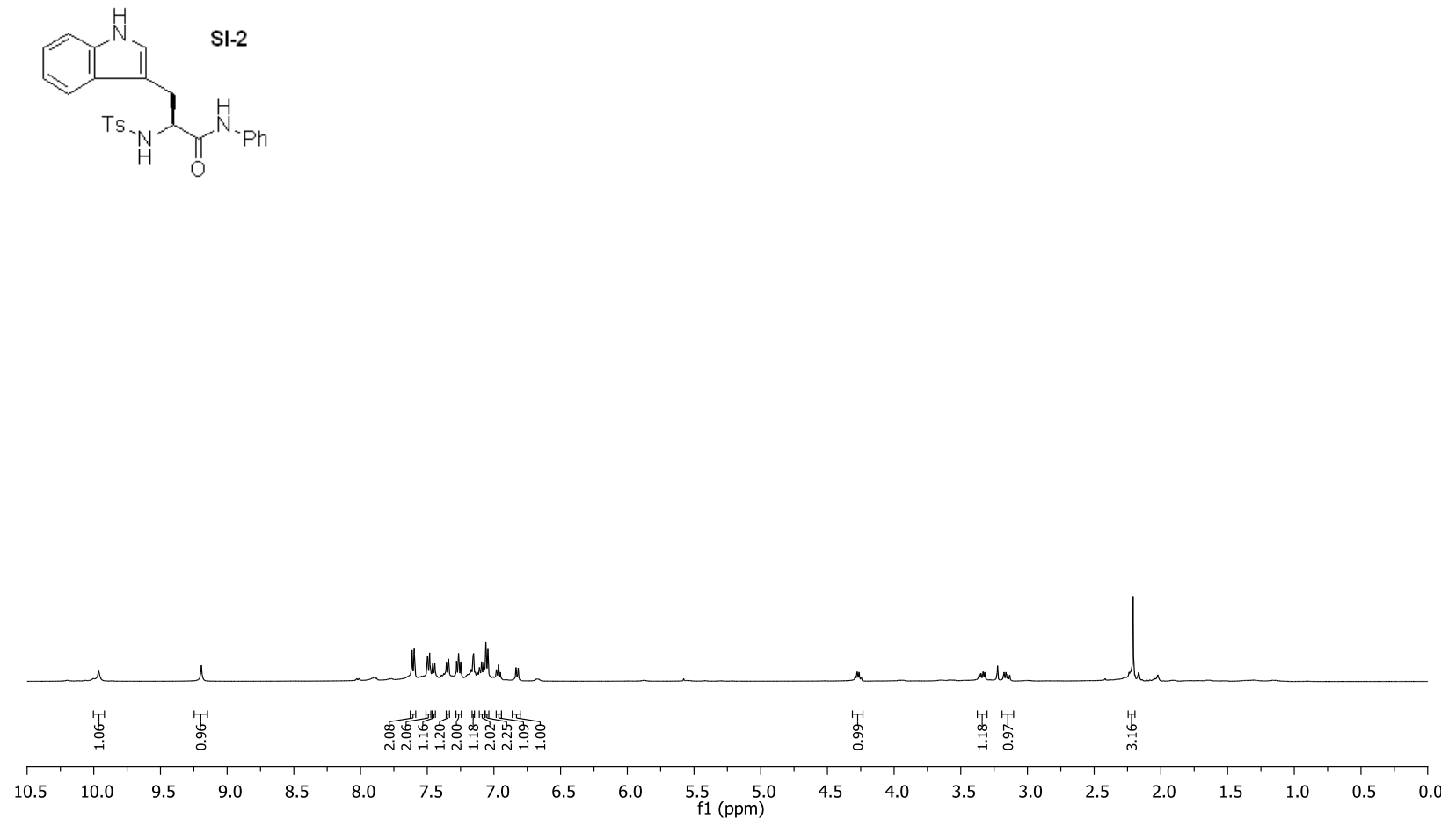

TDM_11_63_1.4C1
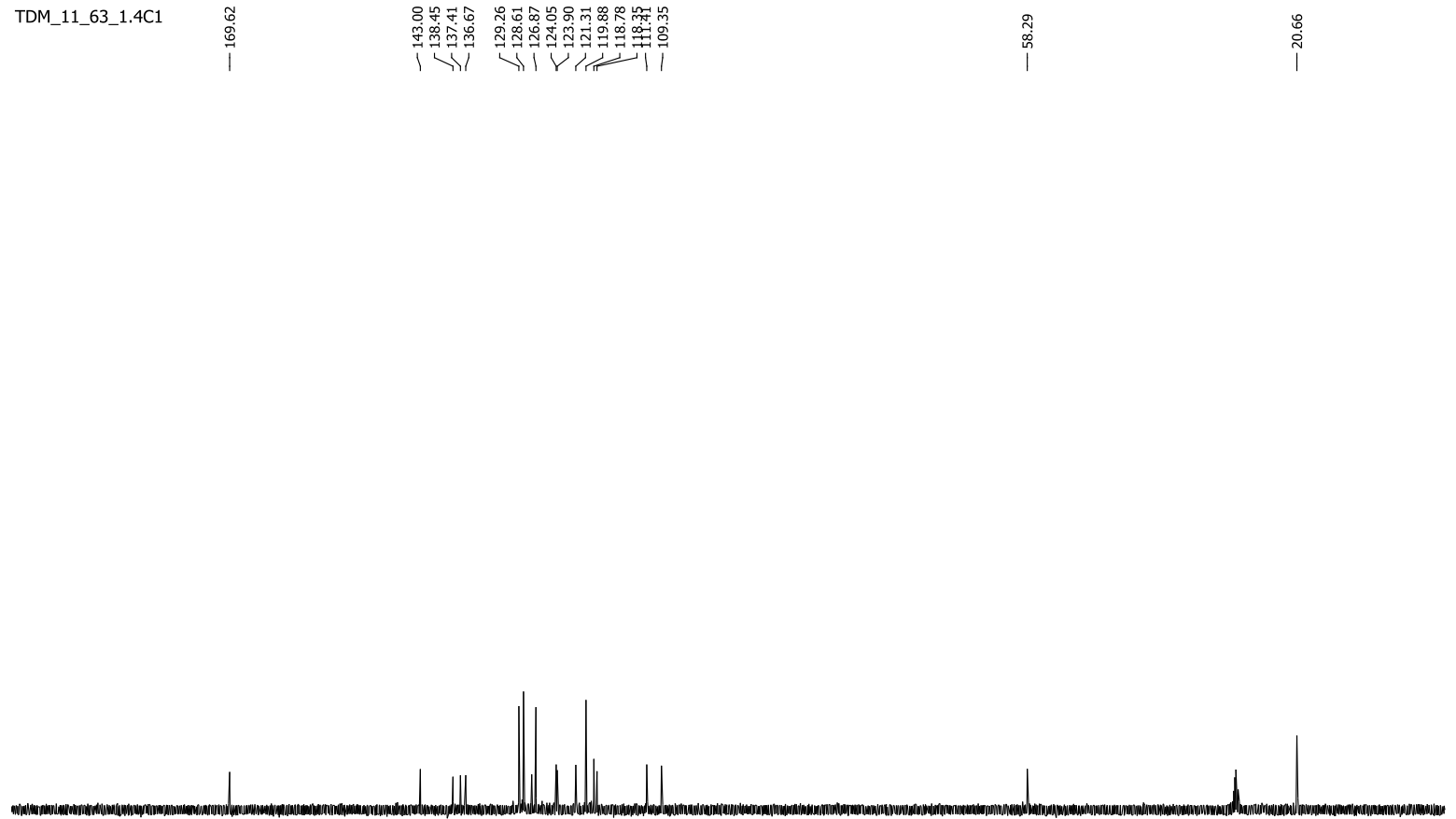

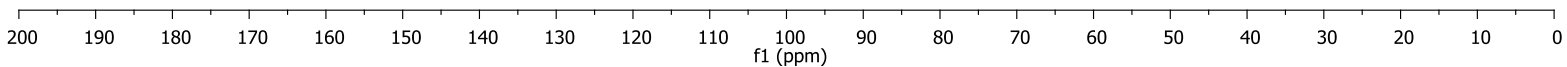


TDM_11_133_1.4

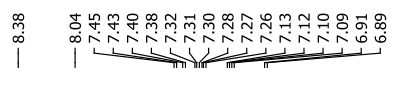

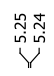
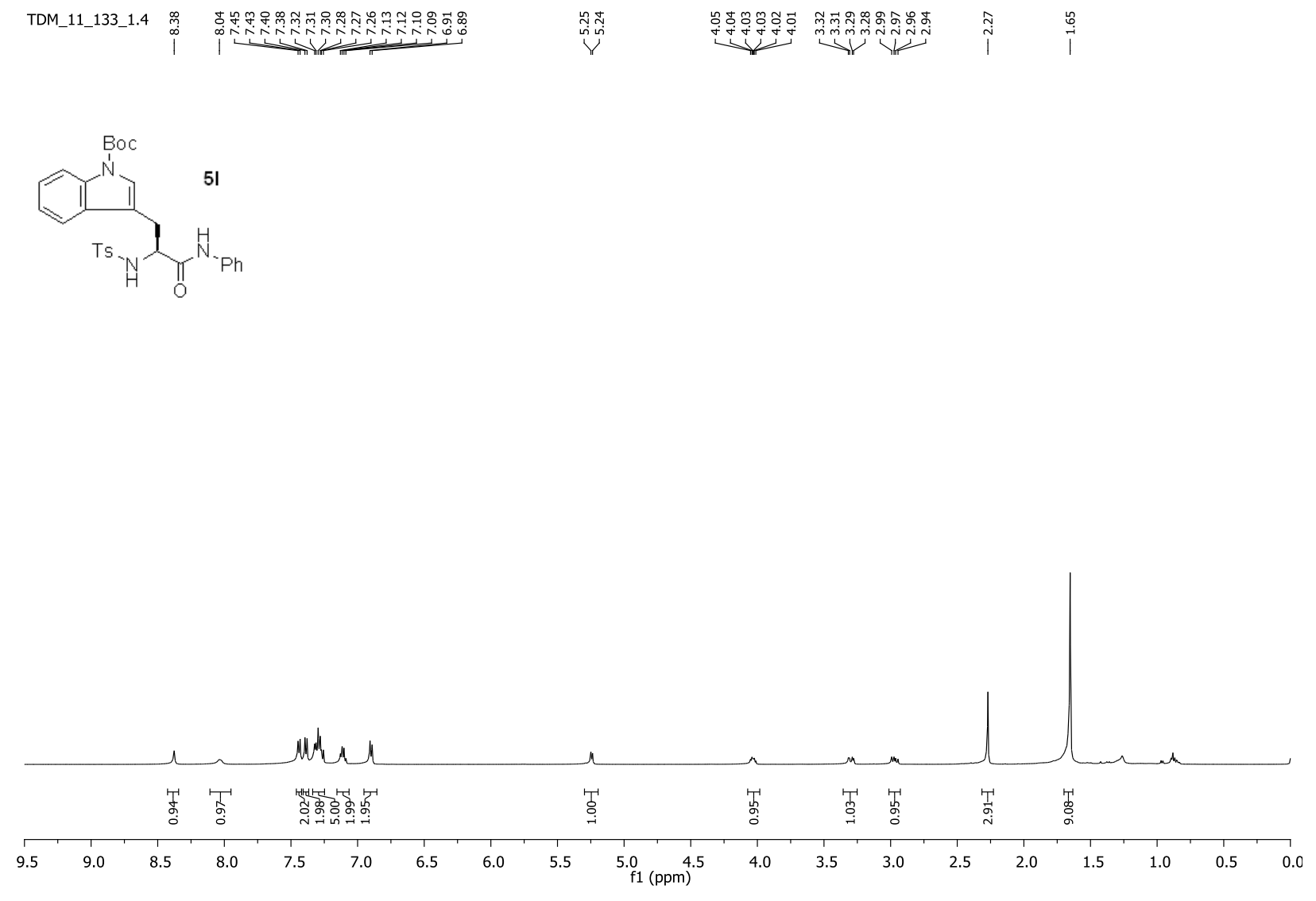

TDM_11_133_1.4C 点

$\underset{\substack { \infty \\ \begin{subarray}{c}{\infty \\ \infty{ \infty \\ \begin{subarray} { c } { \infty \\ \infty } }\end{subarray}}{|c|}$

$\stackrel{\substack{\infty \\ \text { | }}}{\stackrel{n}{N}}$

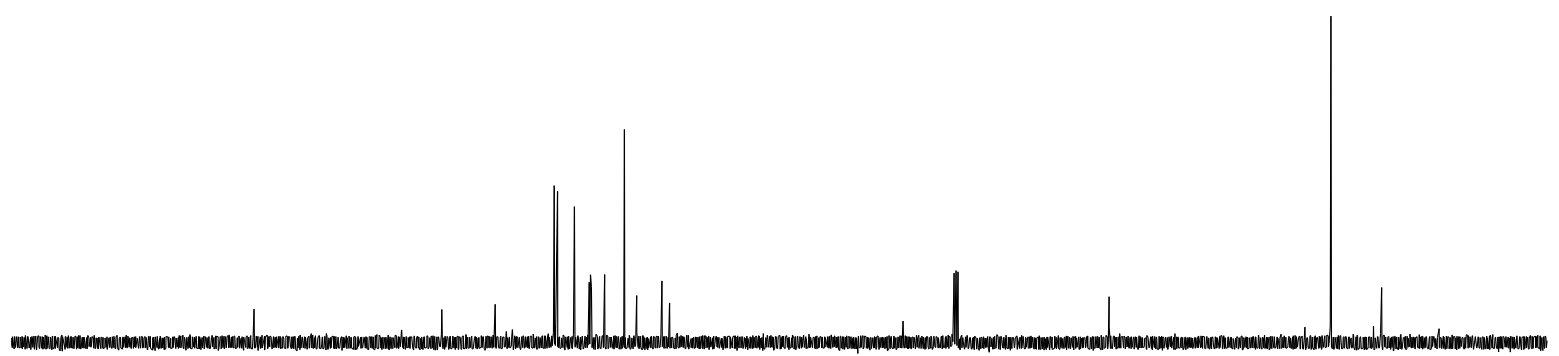

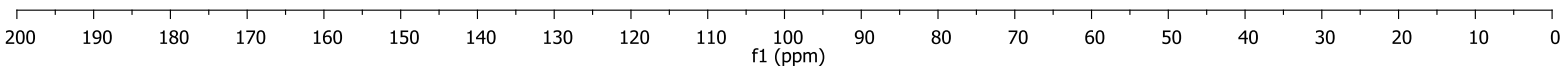


TDM_11_123_1 100
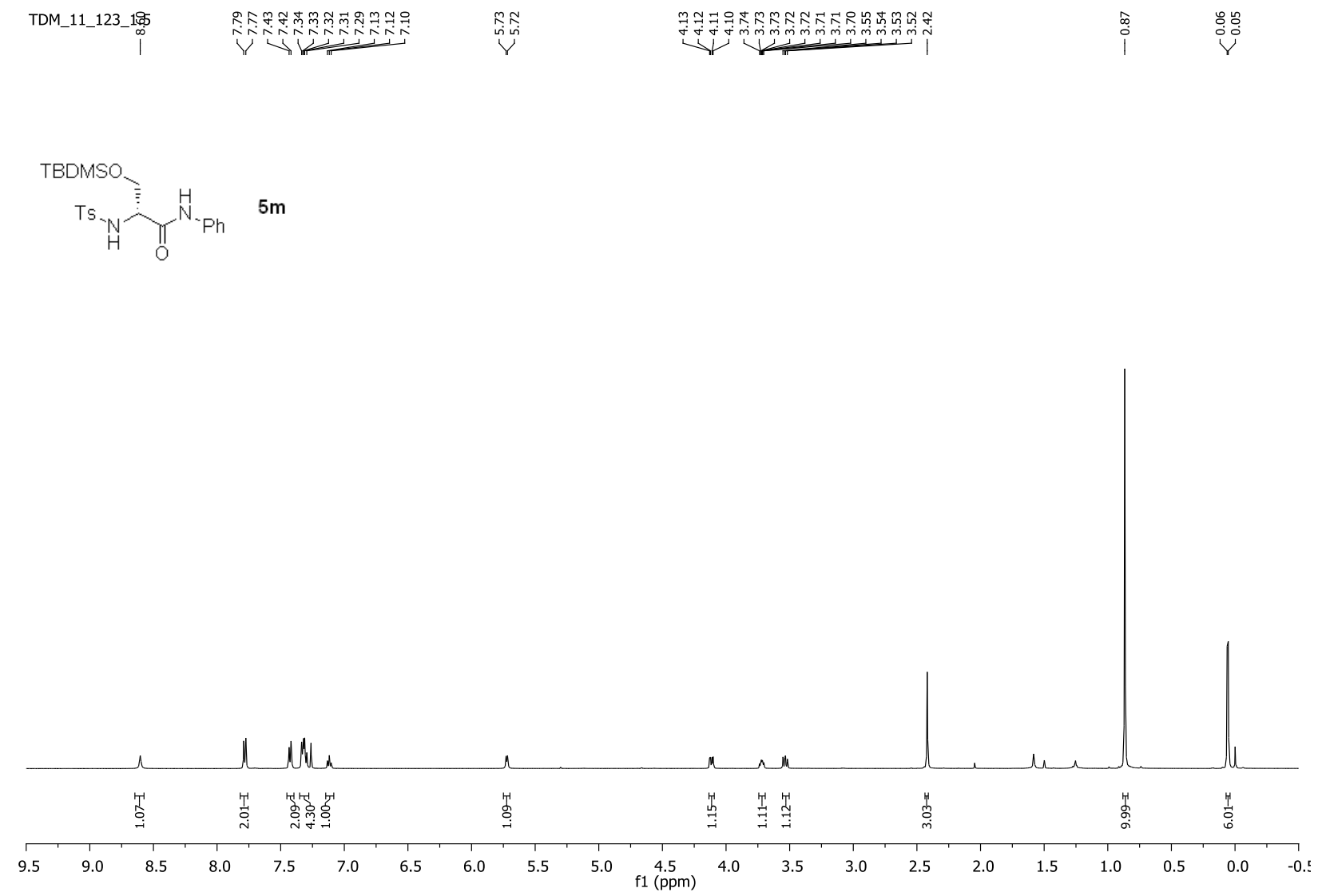

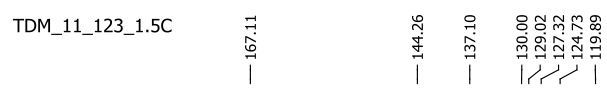

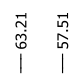

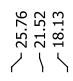

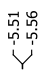

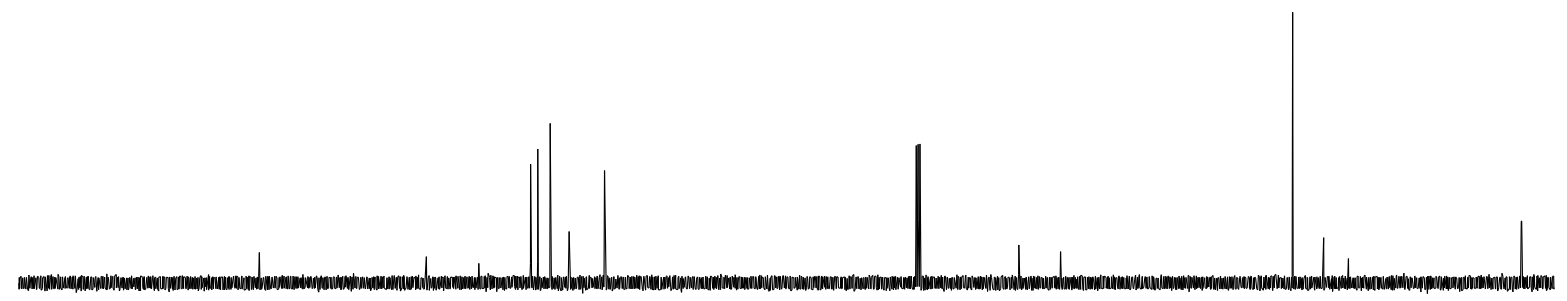

\begin{tabular}{|c|c|c|c|c|c|c|c|c|c|c|c|c|c|c|c|c|c|c|c|c|}
\hline 200 & 190 & 180 & 170 & 160 & 150 & 140 & 130 & 120 & 110 & ${ }_{\mathrm{f} 1}^{100}(\mathrm{p}$ & $\begin{array}{l}90 \\
m)\end{array}$ & 80 & 70 & 60 & 50 & 40 & 30 & 20 & 10 & 0 \\
\hline
\end{tabular}




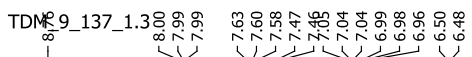

诲

Ns

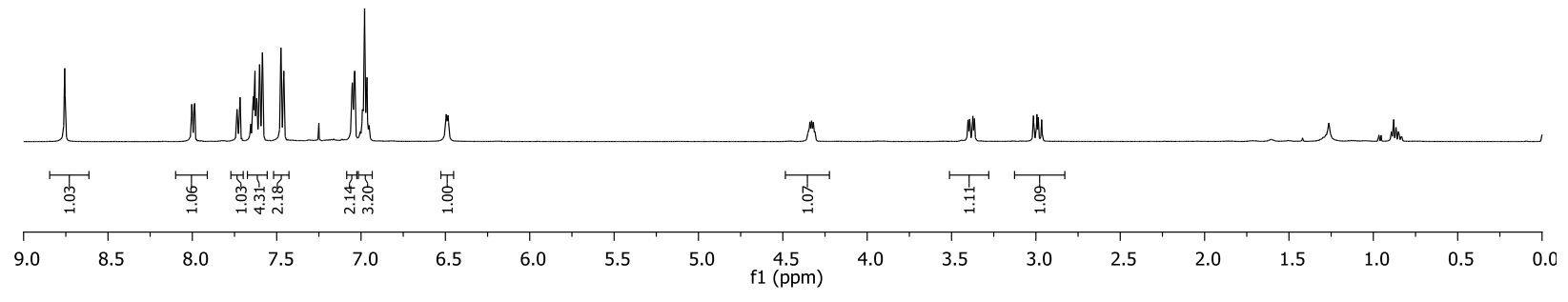

TDM_9_137_1.3C

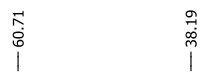
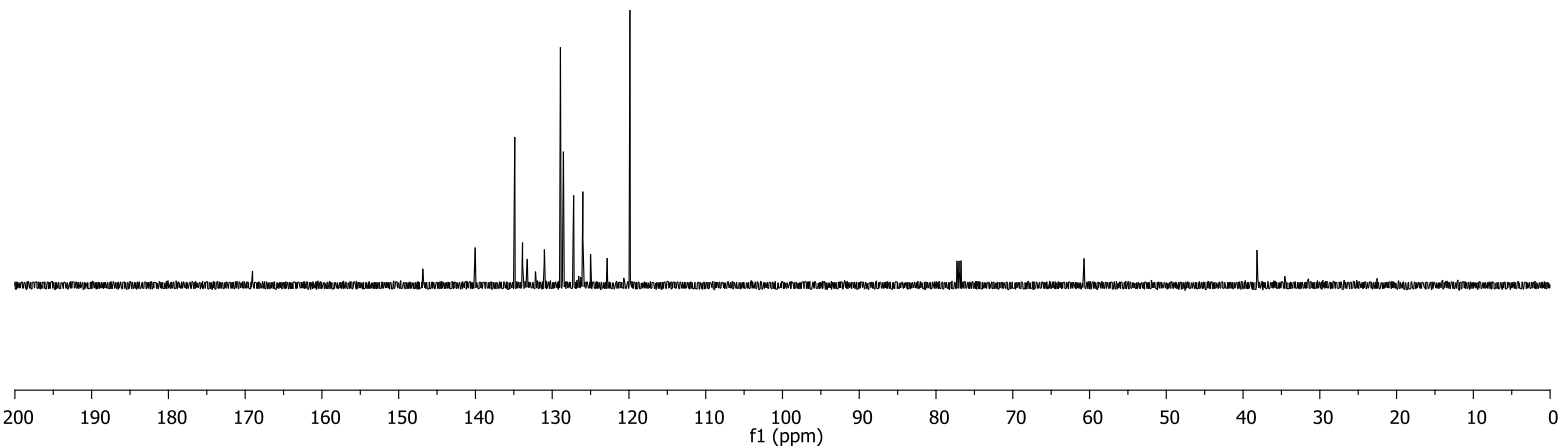


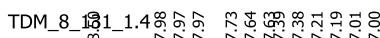

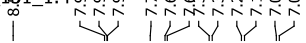<smiles>NN[C@@H](Cc1ccccc1)C(=O)Nc1ccc(Cl)cc1</smiles>

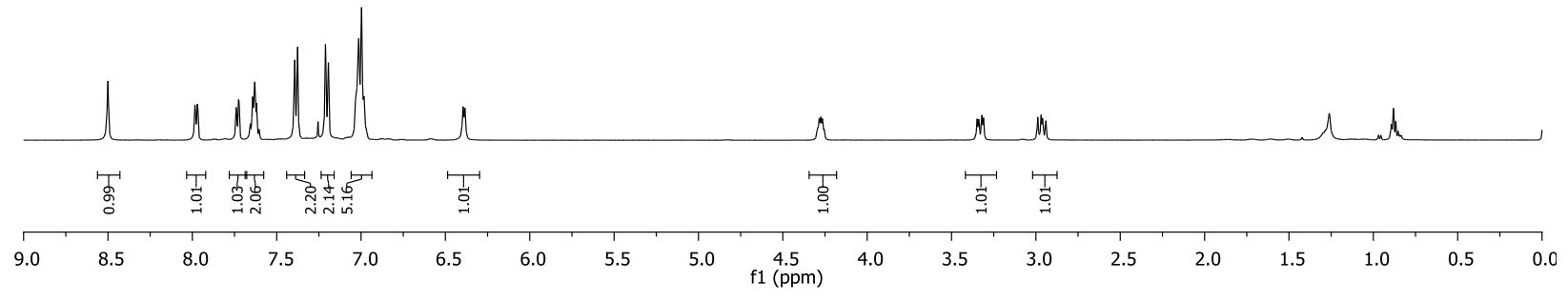

TDM_8_131_1.4C

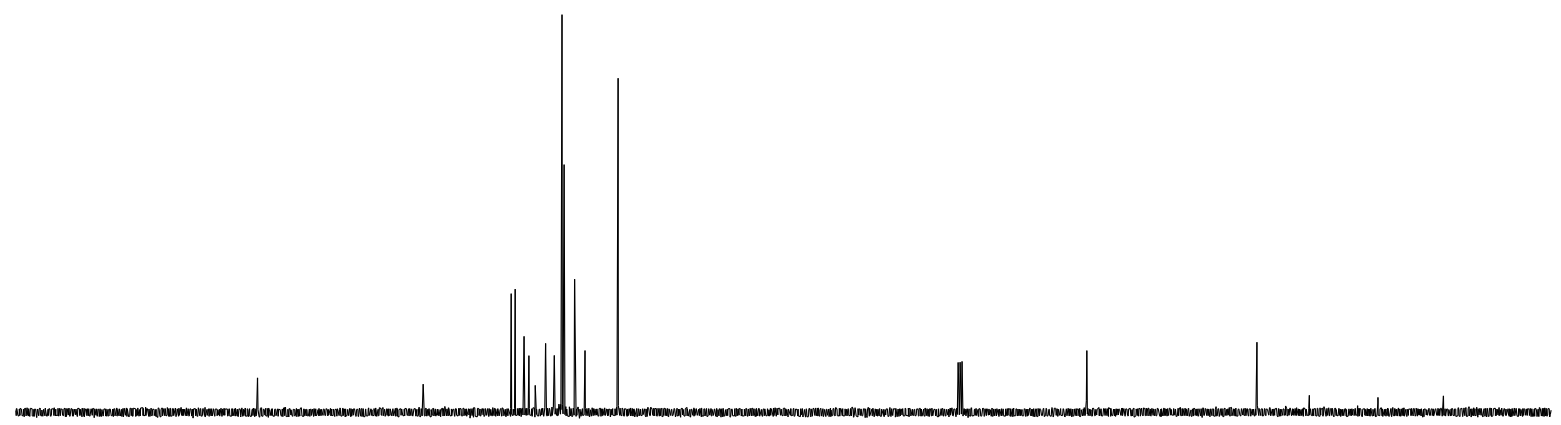

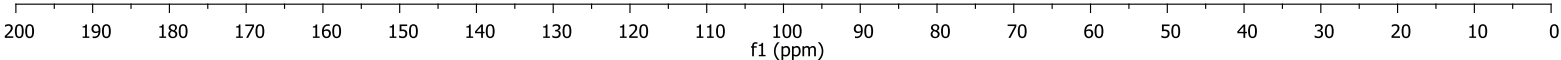




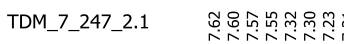

彷궁ํำ

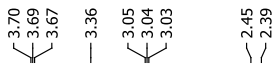

$3 a$

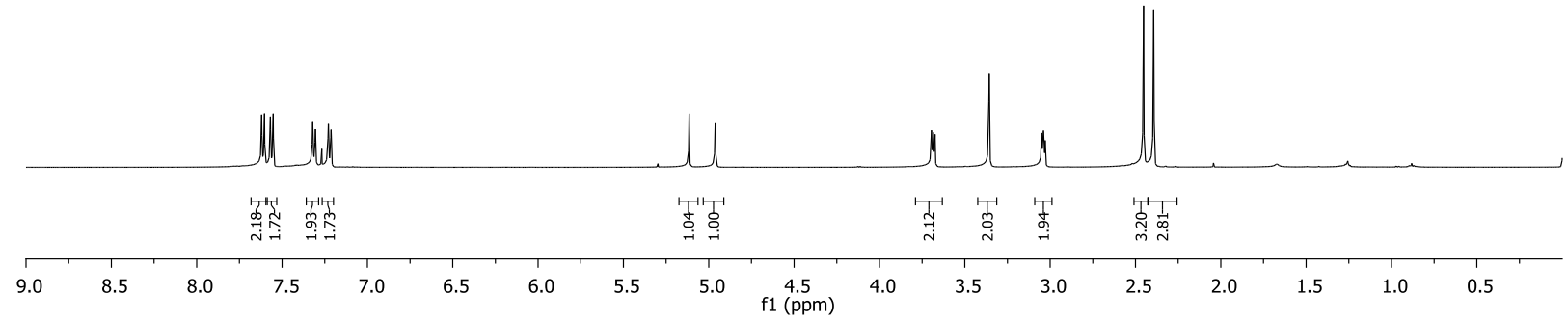

TDM_7_247_2.1C

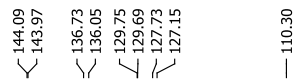

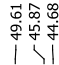

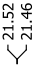

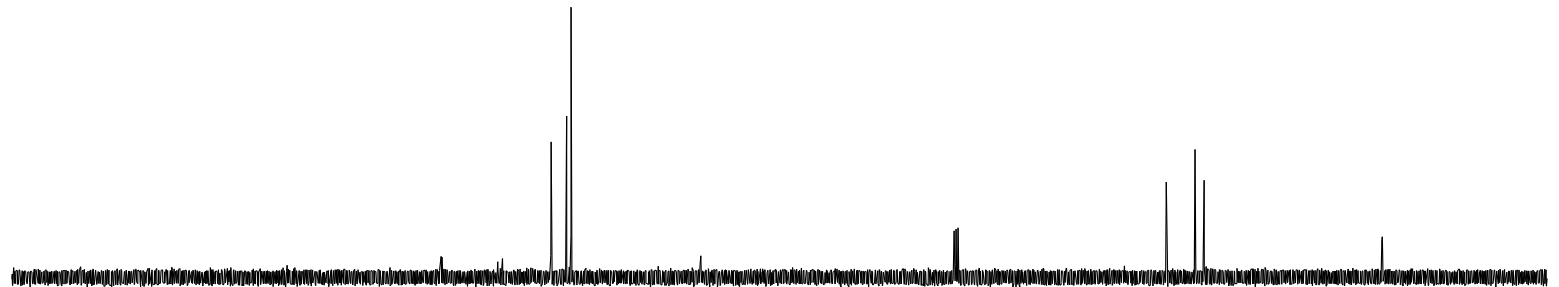

200 $190 \quad 180$ 170 160 
$\mathrm{PhO}_{2} \mathrm{~S}_{3 \mathrm{~b}}^{\mathrm{NO}_{2} \mathrm{Ph}}$
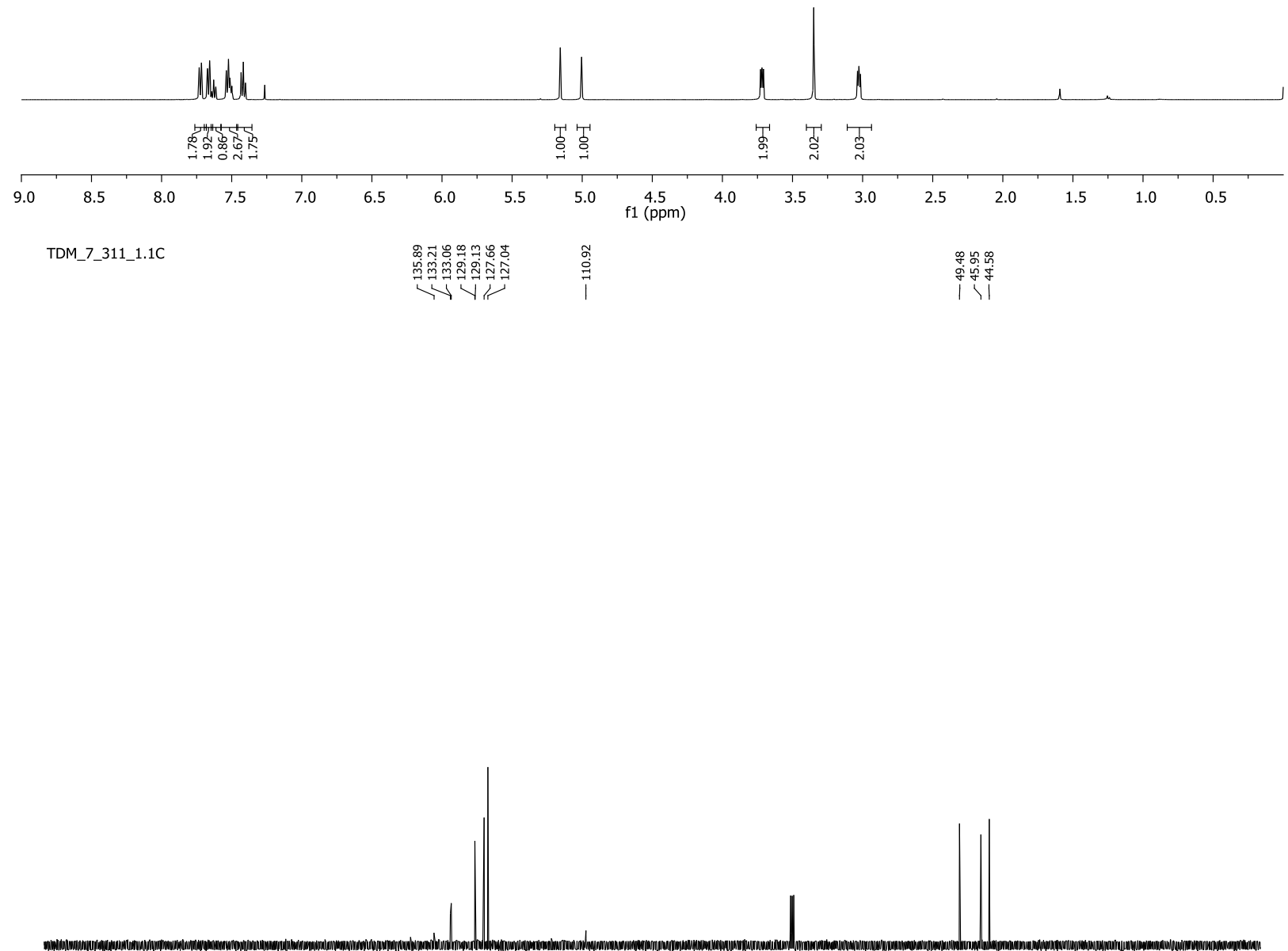

200

$\begin{array}{lllllllll}190 & 180 & 170 & 160 & 150 & 140 & 130 & 120 & 110\end{array}$
100
$\mathrm{f} 1(\mathrm{ppm})$ $90 \quad 80$ $70 \quad 60$ 
TDM_11_71_1.1

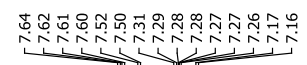

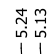

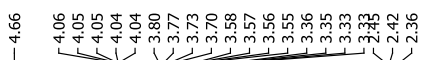

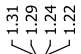

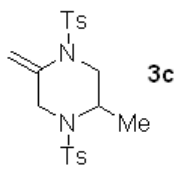

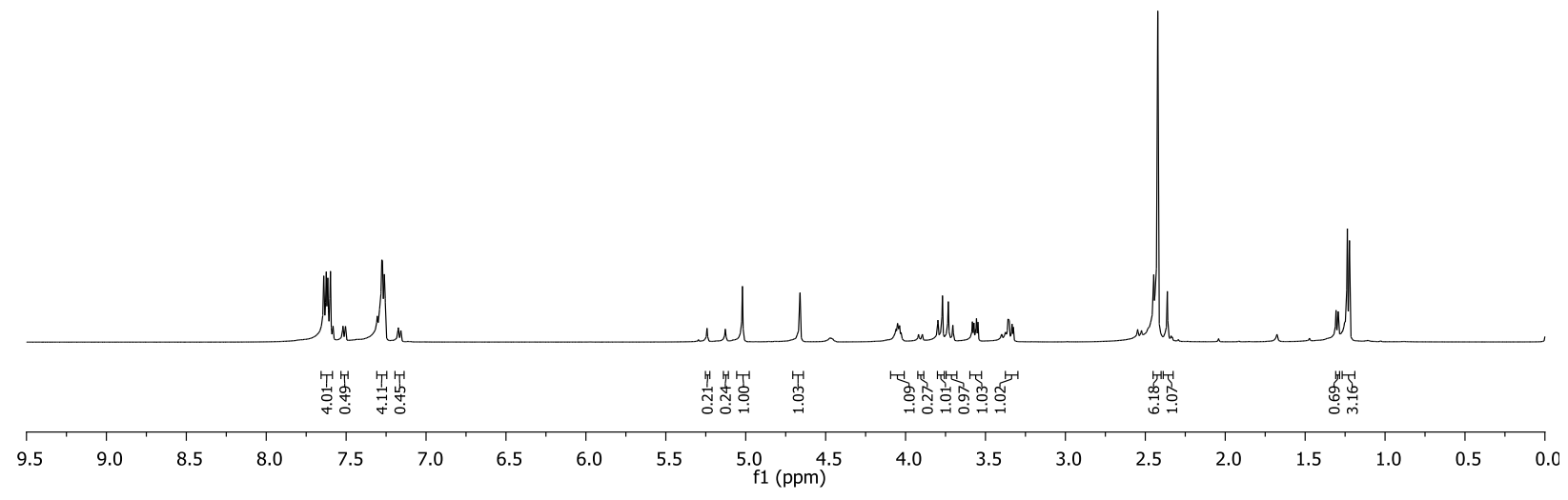

TDM_11_71_1.1C

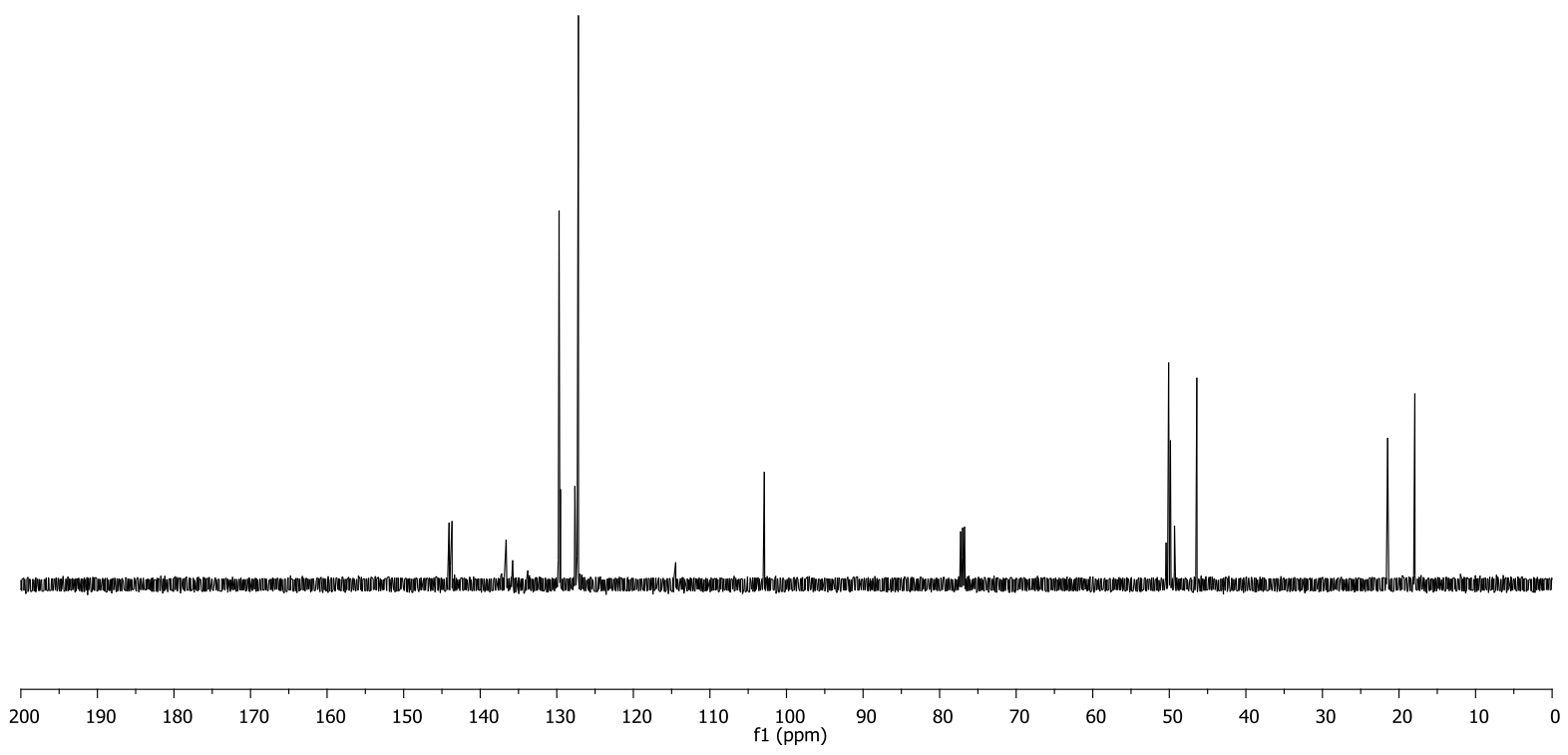




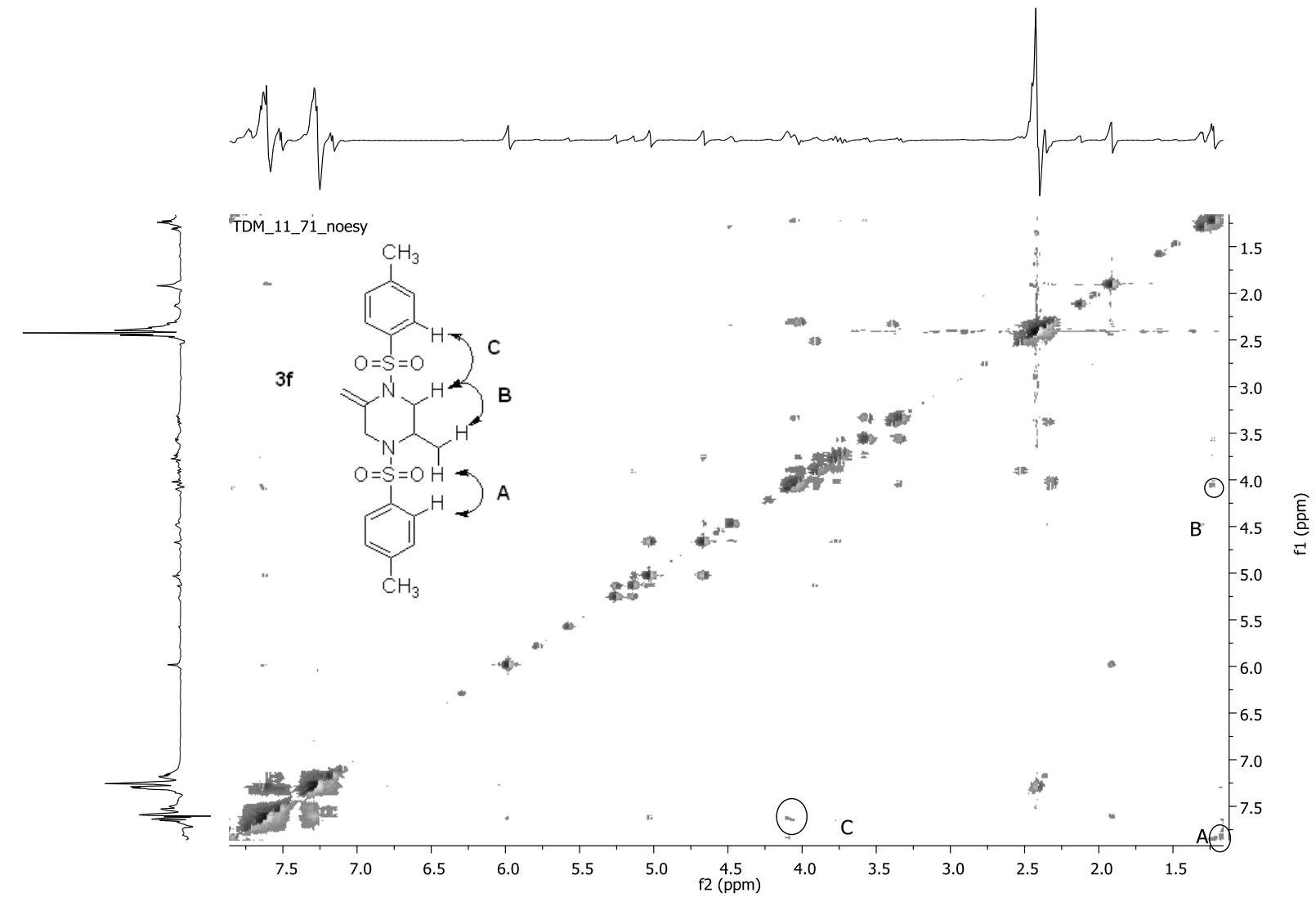


TDM_11_81_1.2

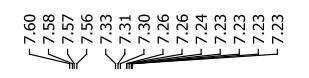

i.

$\overbrace{\substack{T^{2} \\ T}}^{T s} 3 d$

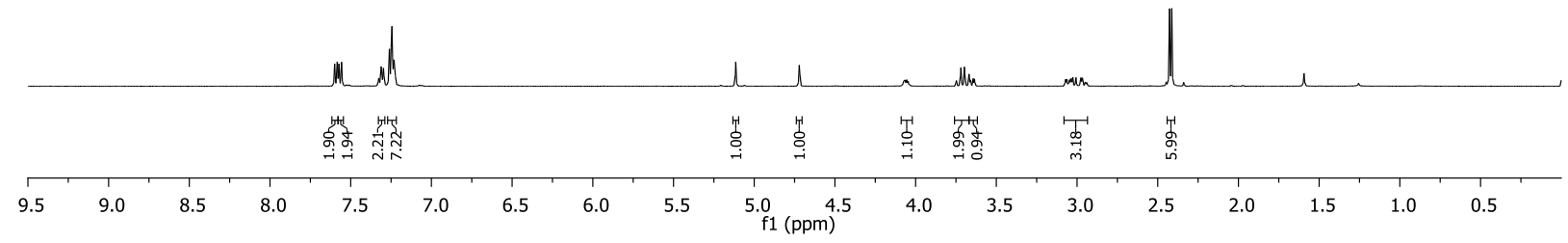

TDM_11_81_1.2C

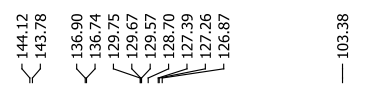
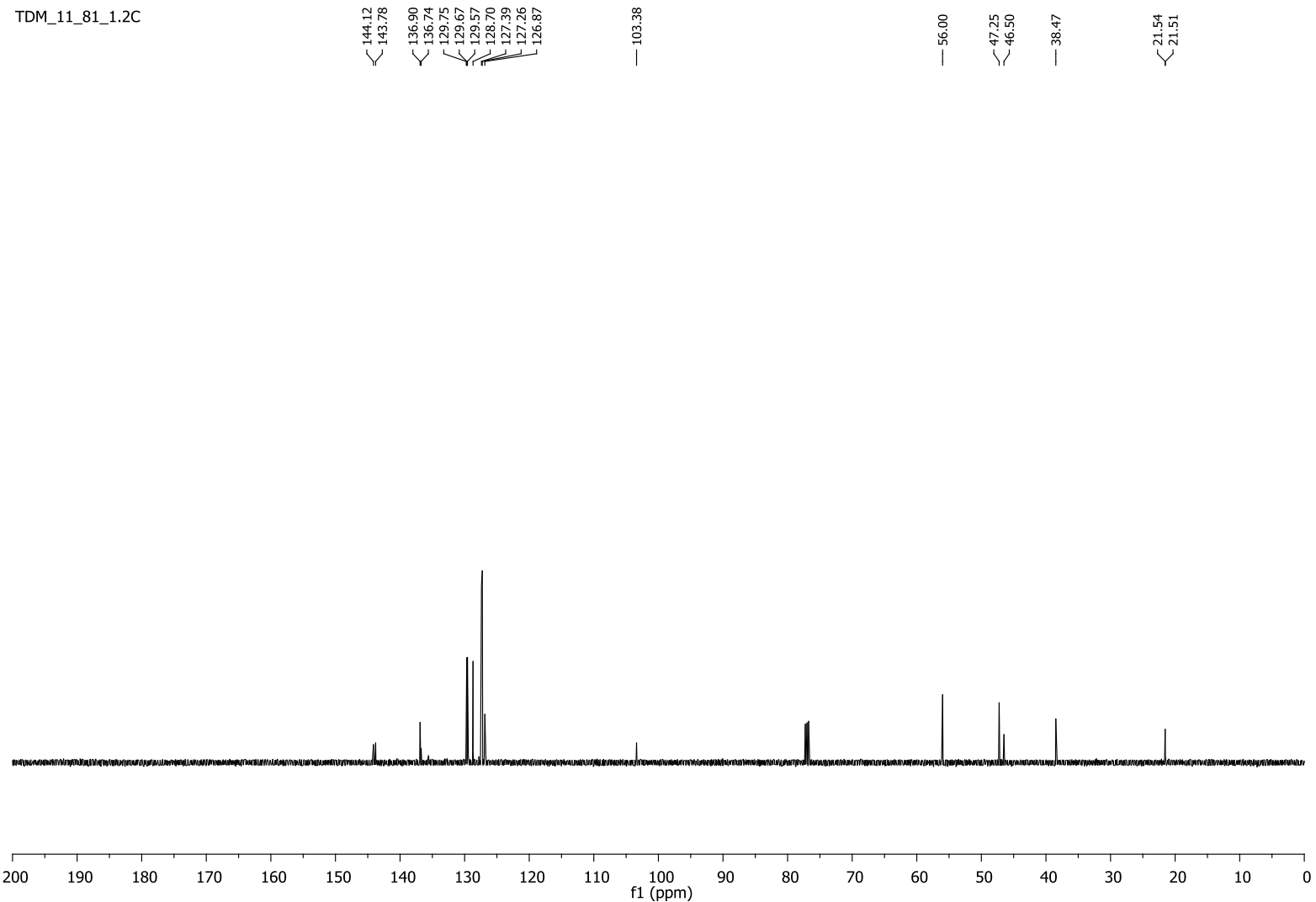
TDM_12_5_1.2

$\overbrace{T^{N}}^{N} \overbrace{\mathrm{Ph}}^{N^{-T s}} 3 \mathrm{e}$

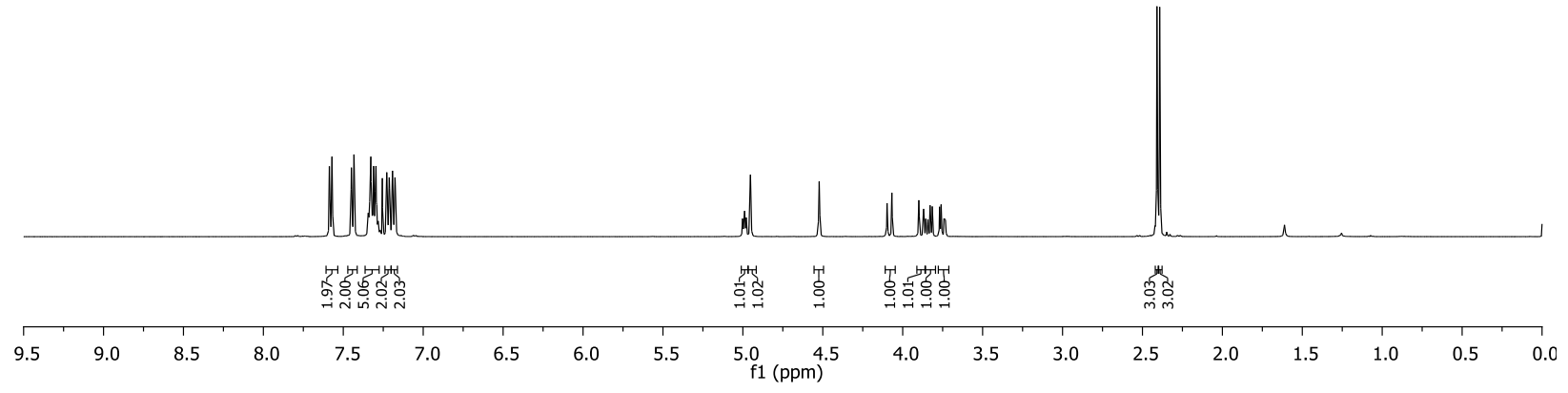

TDM_12_5_1.2C

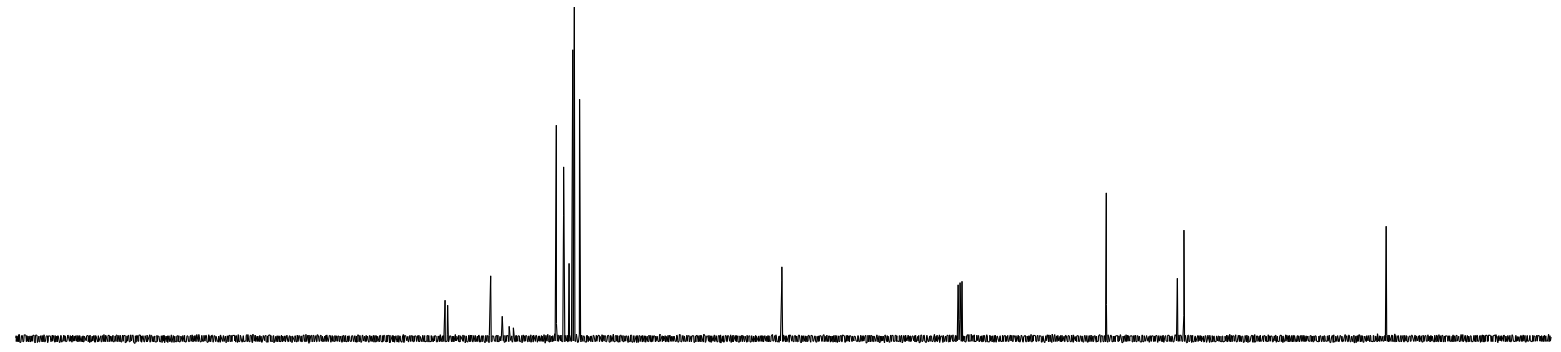


TDM_11_131_1.1

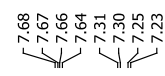

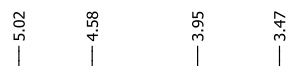

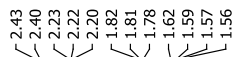<smiles>[3H]N1CC2(CCCC2)N([3H])CC1=C</smiles>

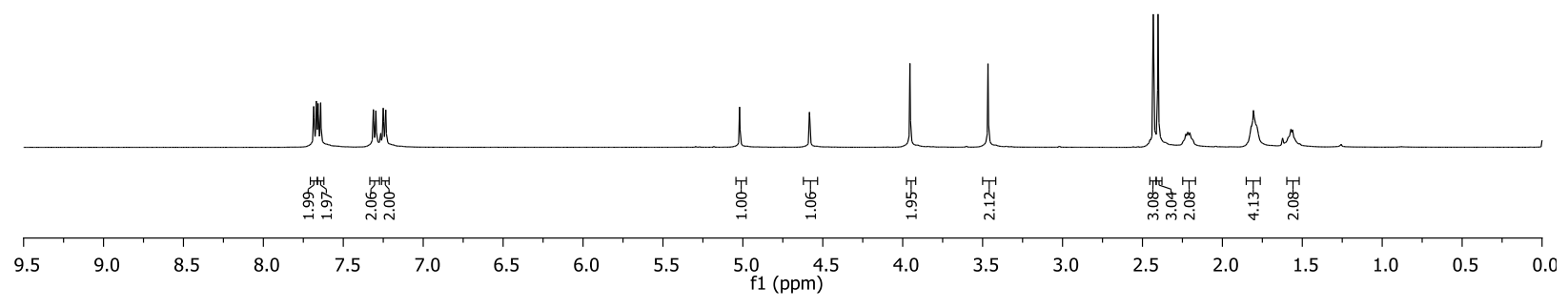

TDM_11_131_1.1C

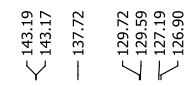

$\mathfrak{N}$
$\stackrel{0}{0}$
I

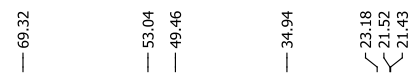

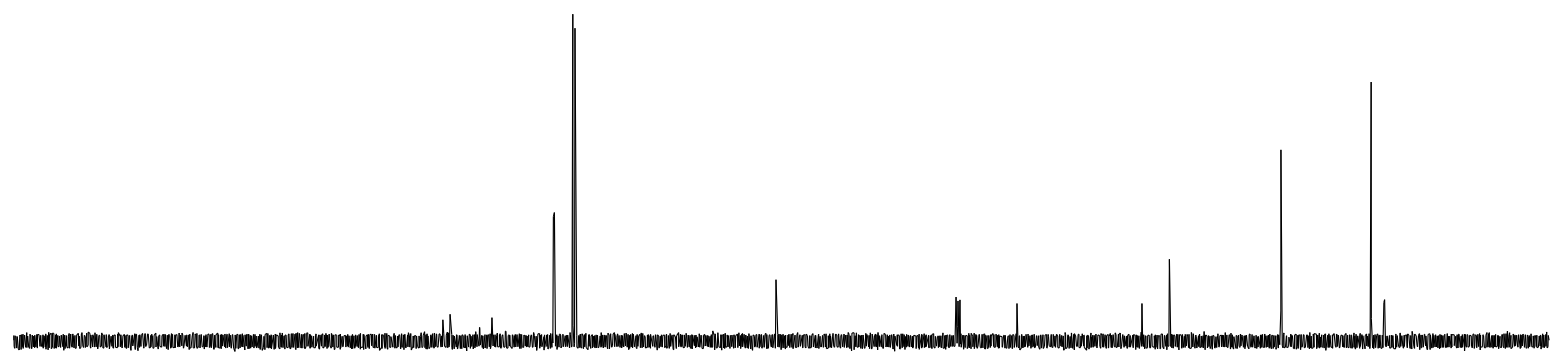


$3 g$

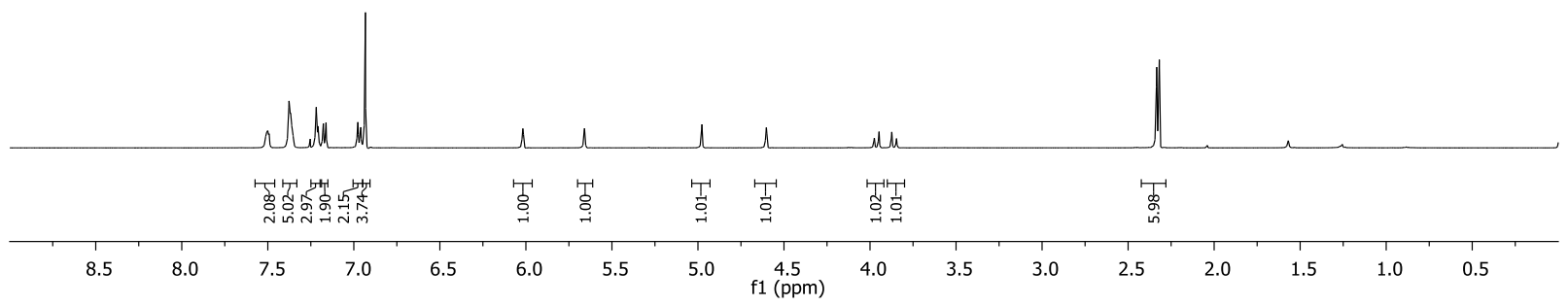

TDM_11_3_1C

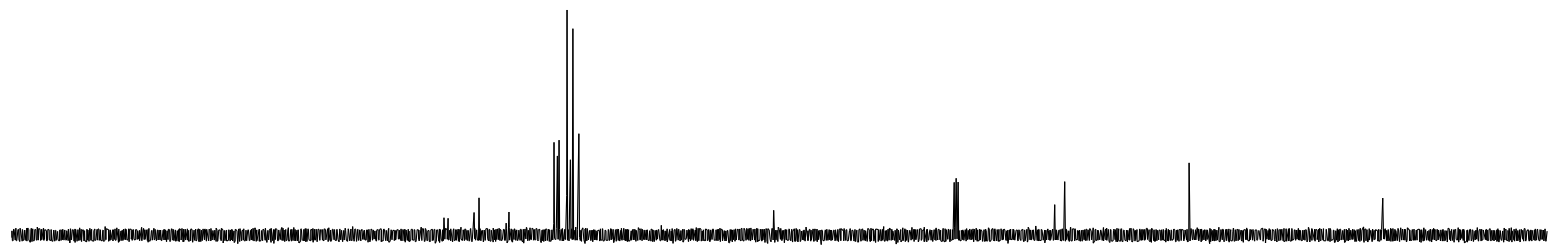


TDM_7_271_2.1

Y
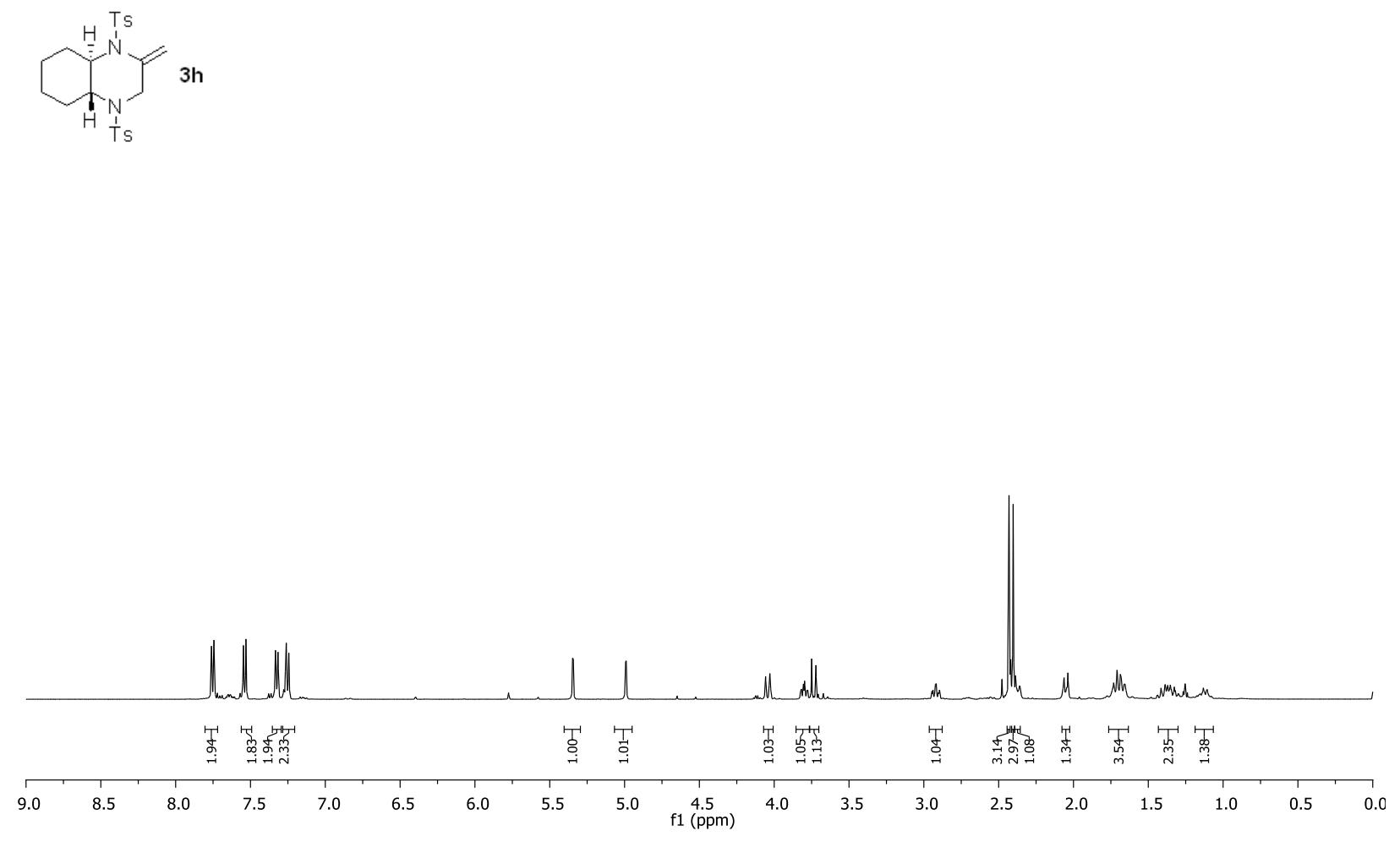

TDM_7_271_2.1C

|
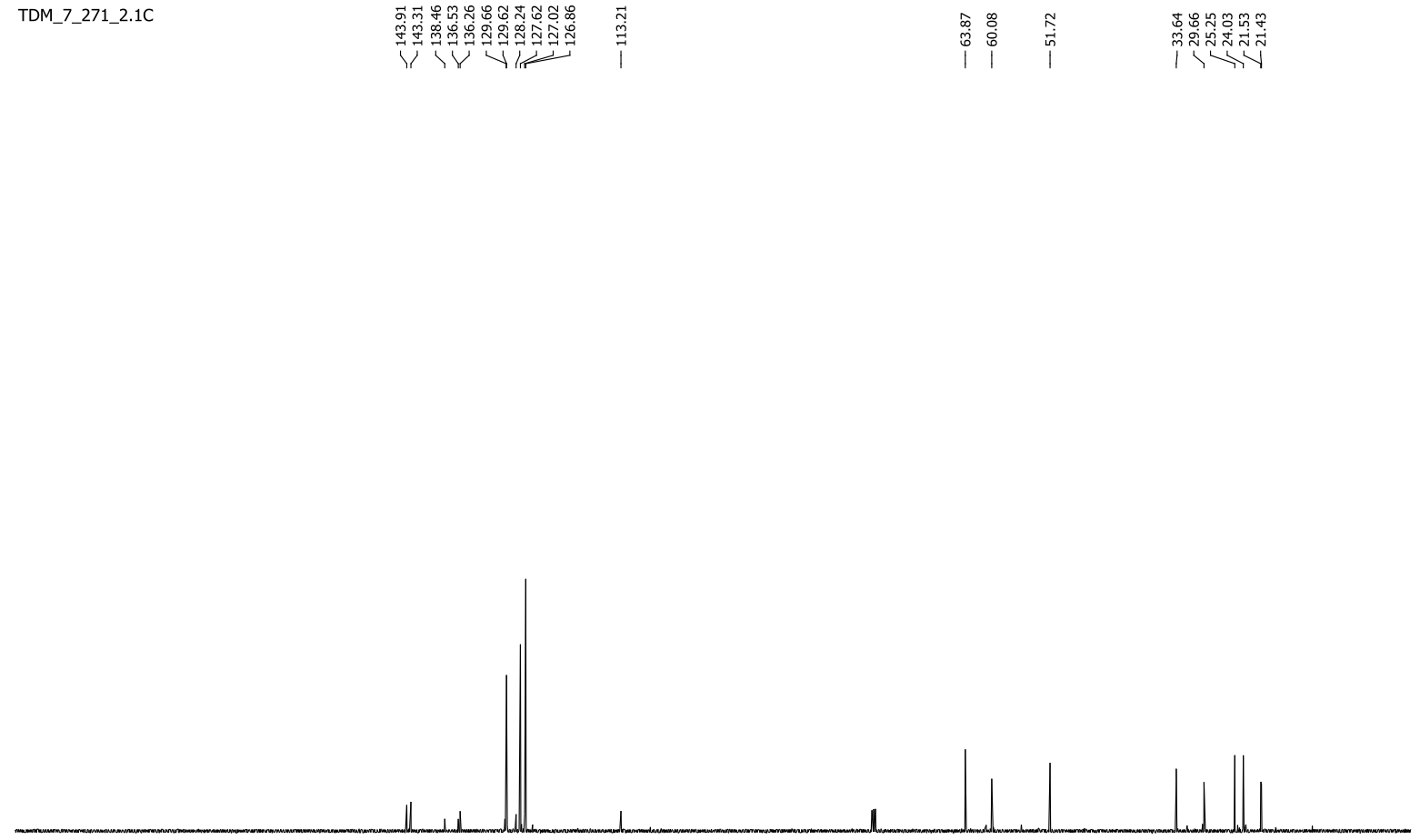


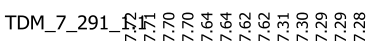

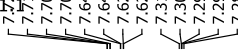

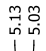

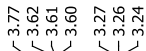

บิ

$\sum_{\text {Ts }}^{\text {Ts }} 3 \mathbf{i}$

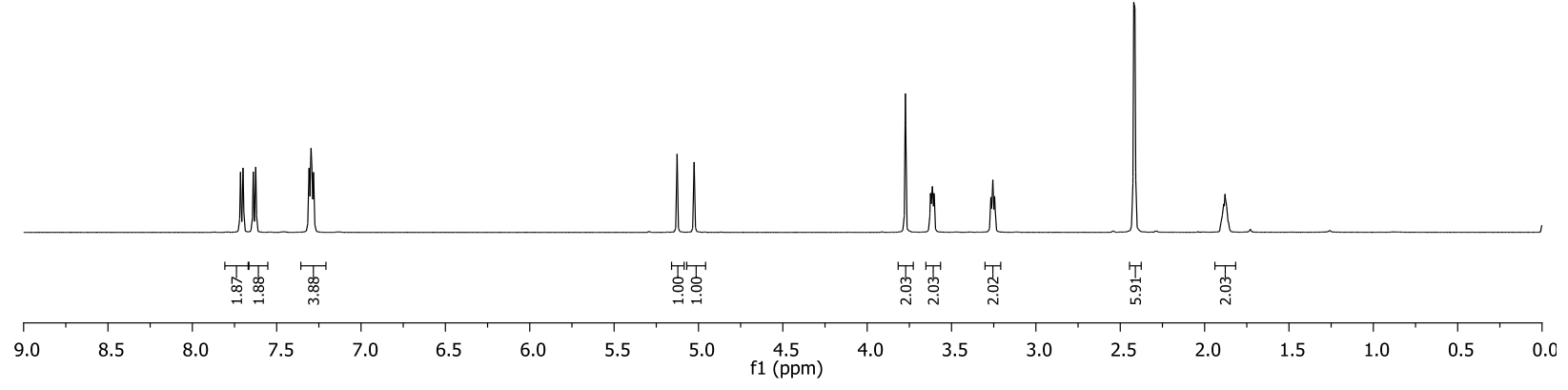

TDM_7_291_1.1C

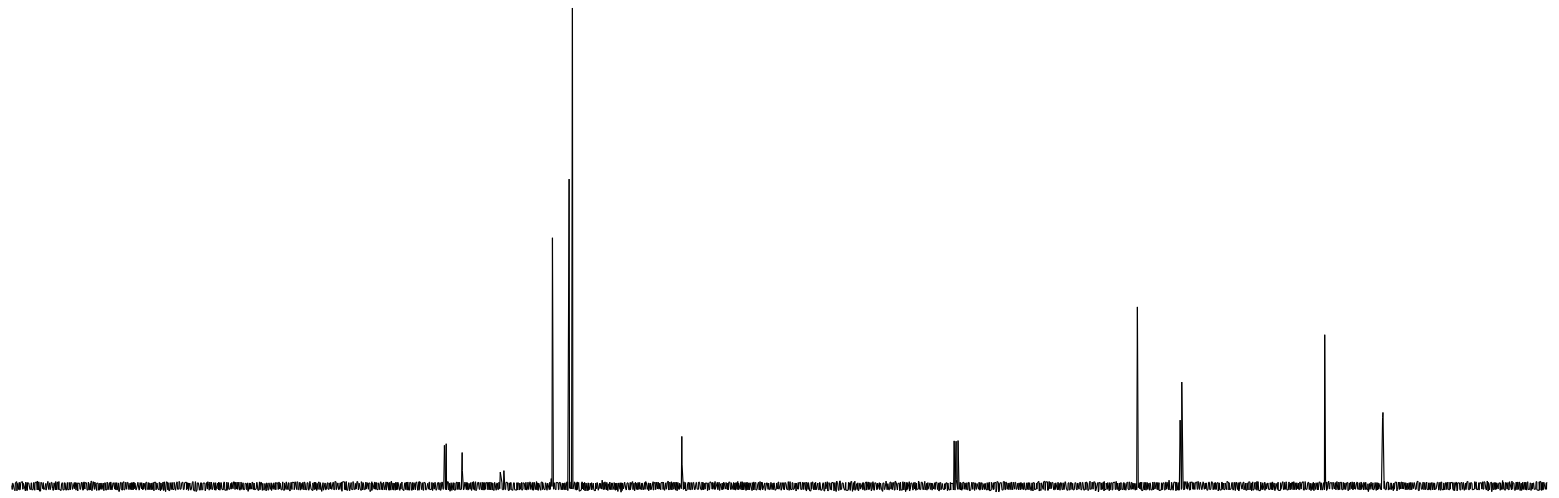

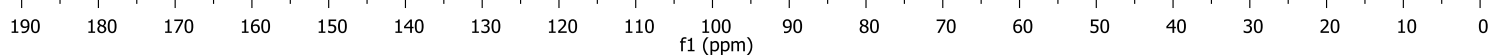


TDM_11_65_1

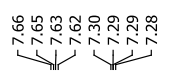<smiles>C=C1CN([13CH3])CC(C)(C)C1</smiles>

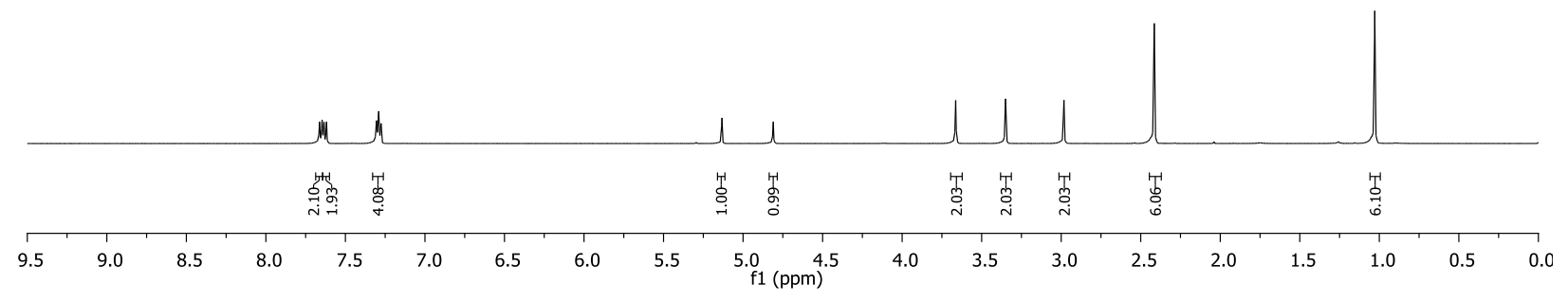

TDM_11_65_1C

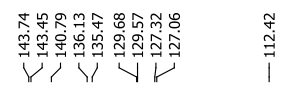
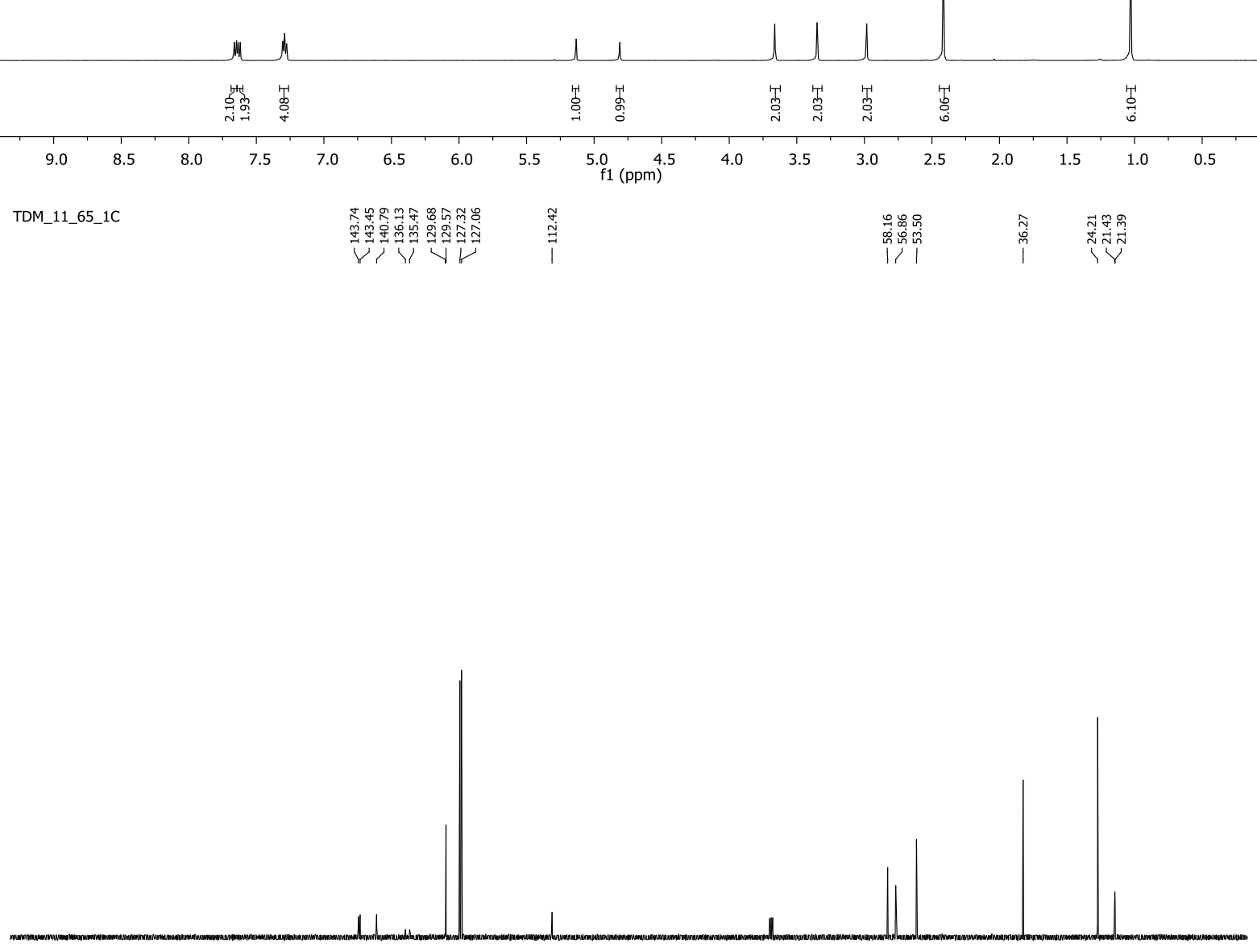

$190 \quad 180$ 
TDM_8_21_2

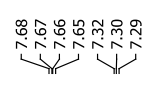

$\overbrace{i s}^{N s} 3 k$

\section{i}

\section{i}

|

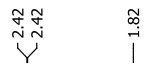
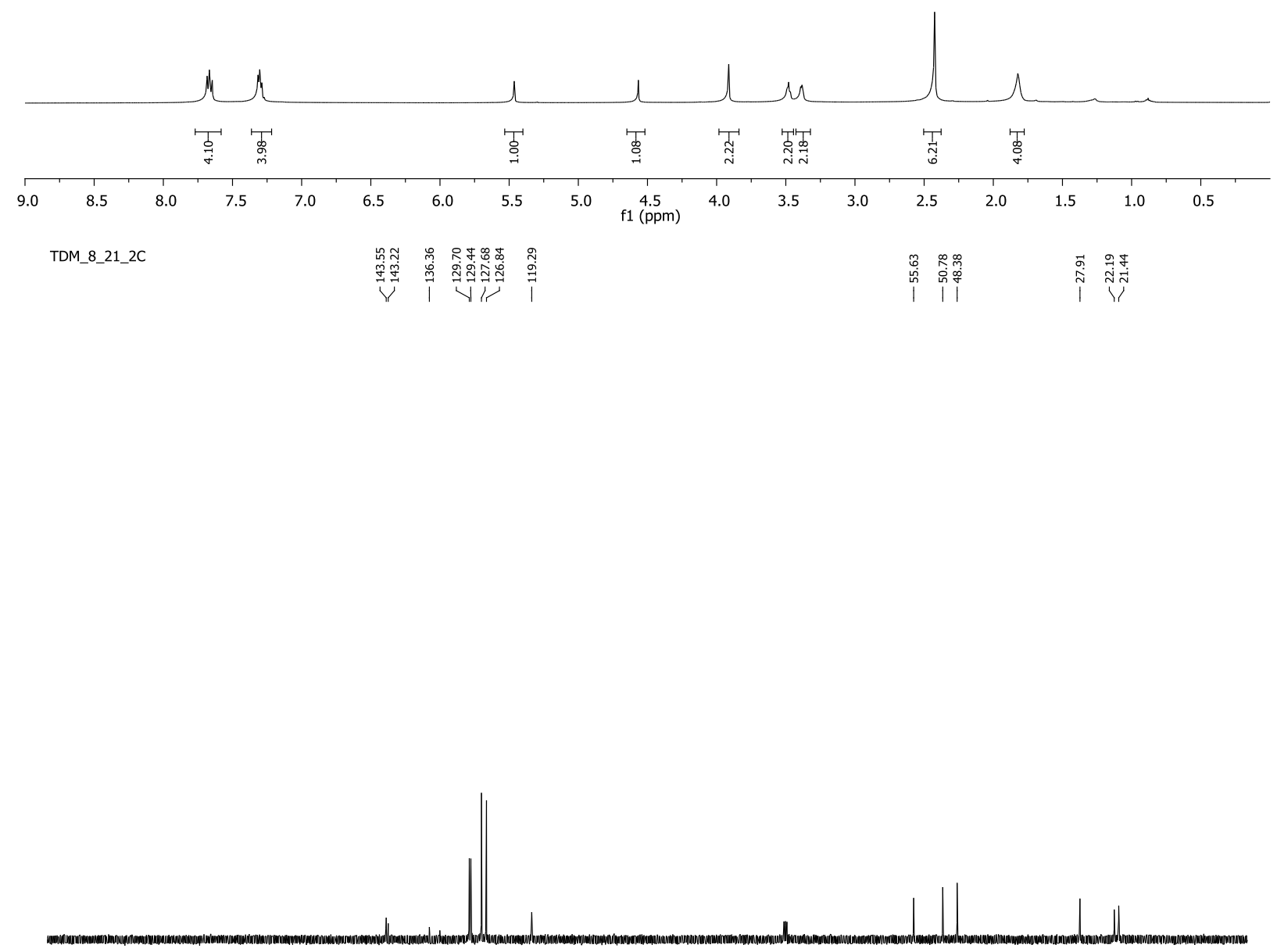

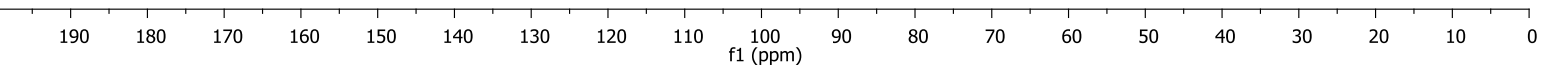


<smiles>C=C1CN(C(=O)OCC)c2ccccc2N1C(=O)OCC</smiles>

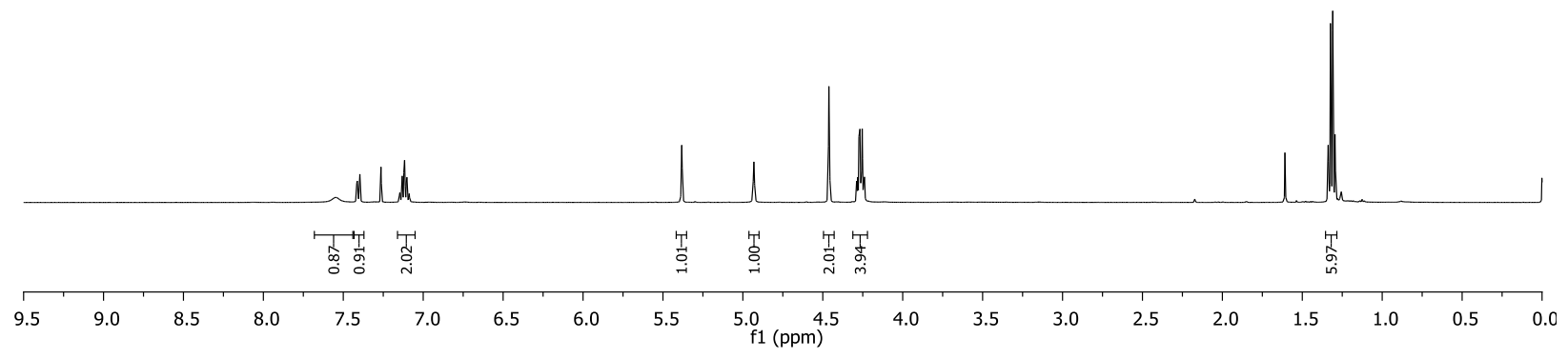

TDM_11_79_1.1C

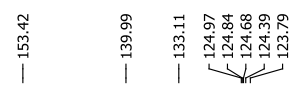
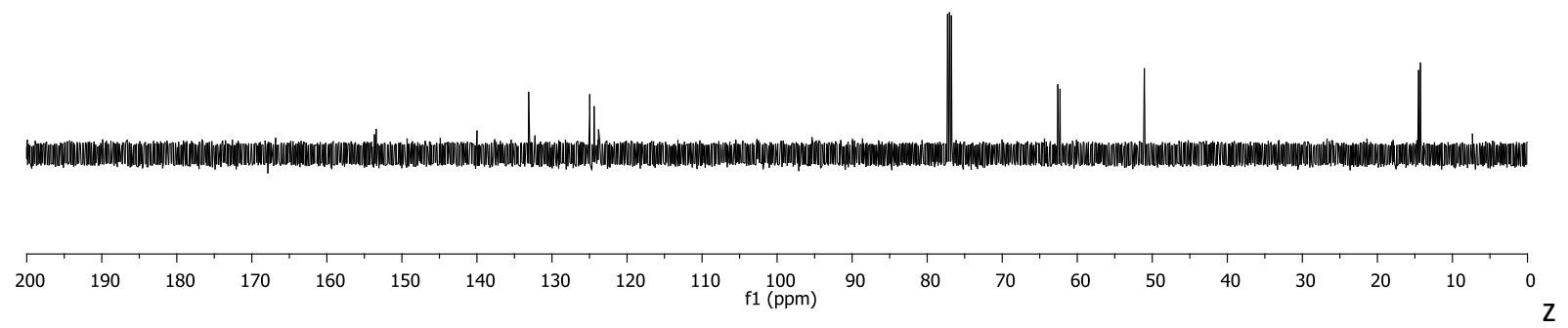

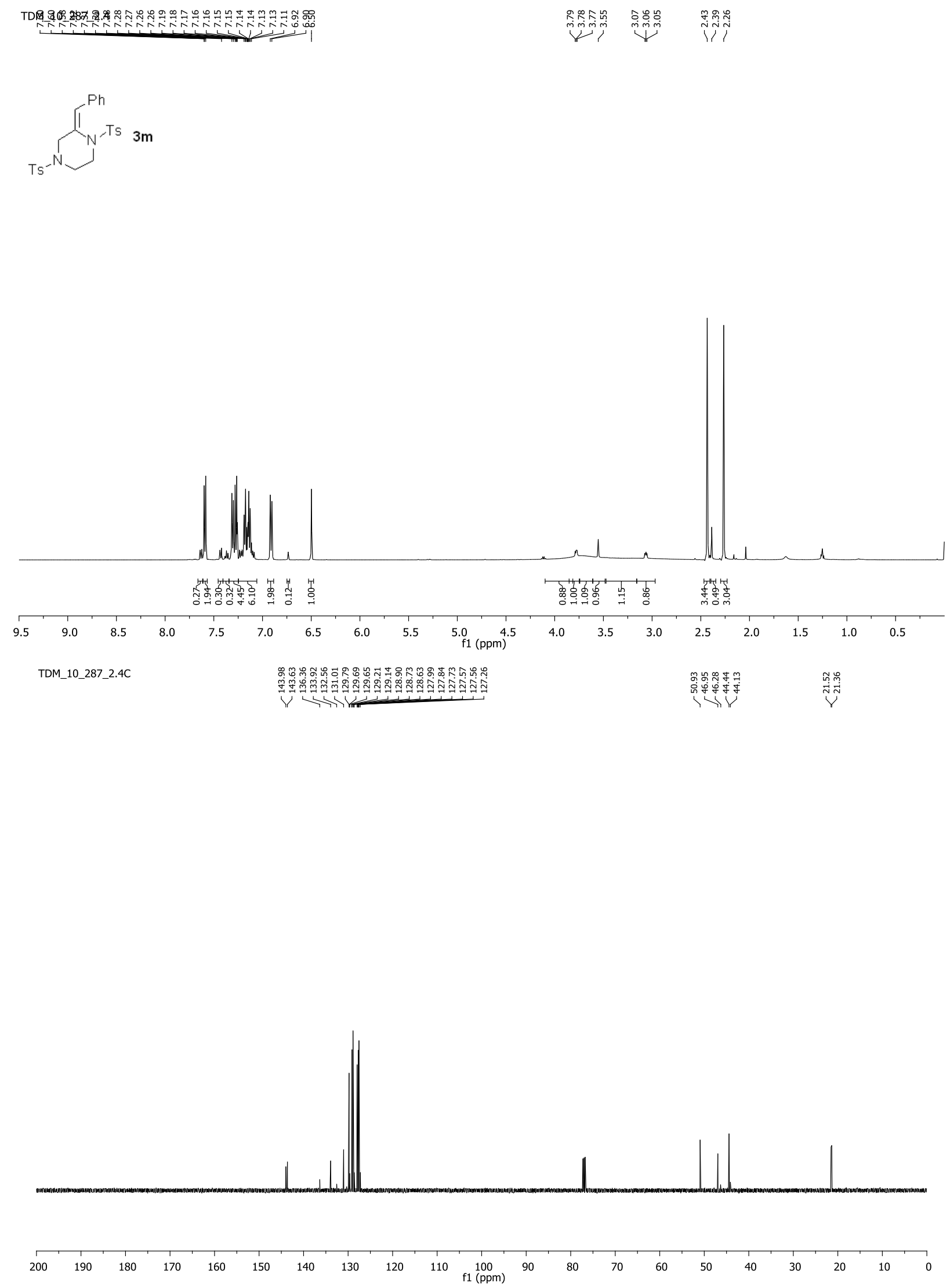
TDM_10_287_noesy

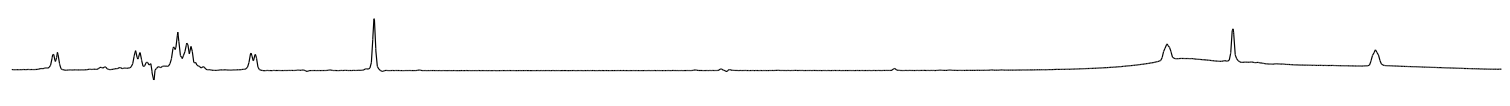

TDM_10_287_noesy
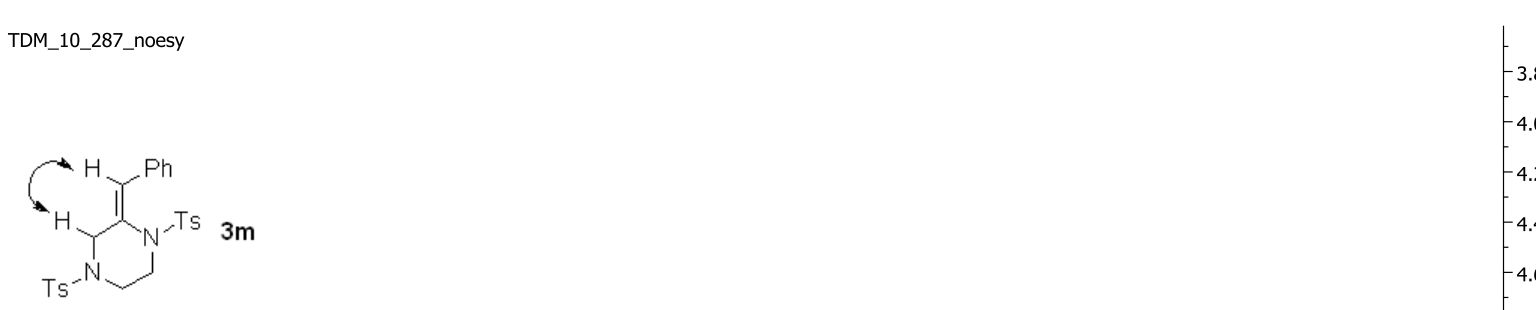
$3 n$

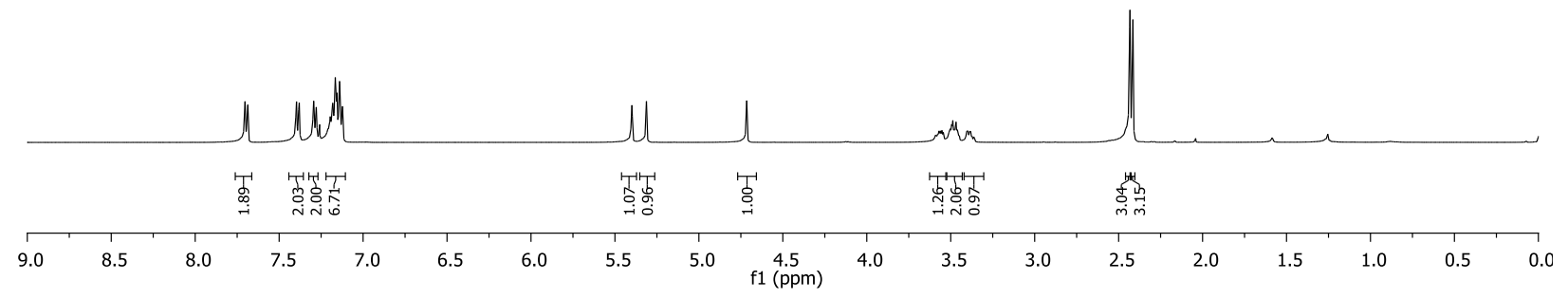

TDM_10_287_2.3C

|

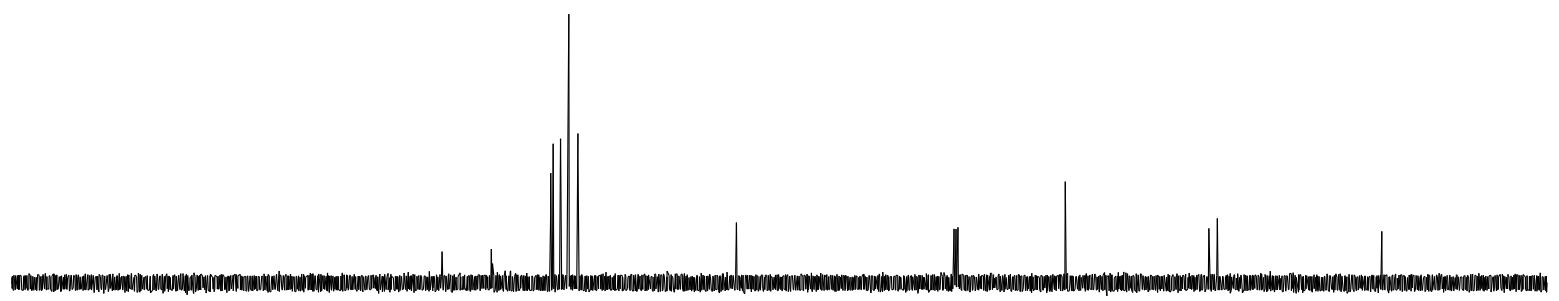




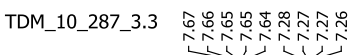

30

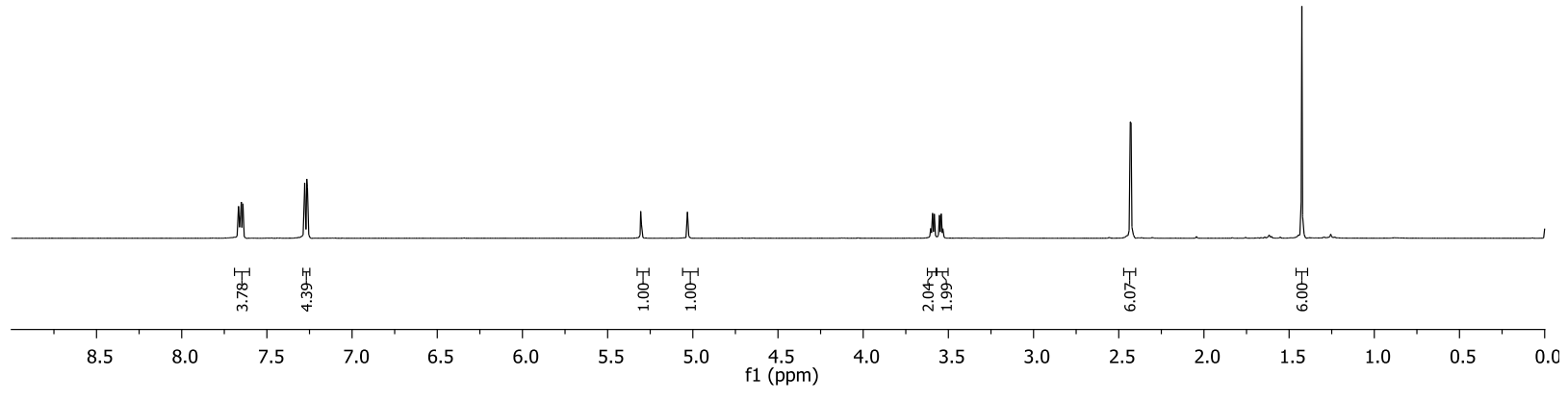

TDM_10_287_3.3C

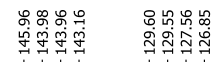

บ난
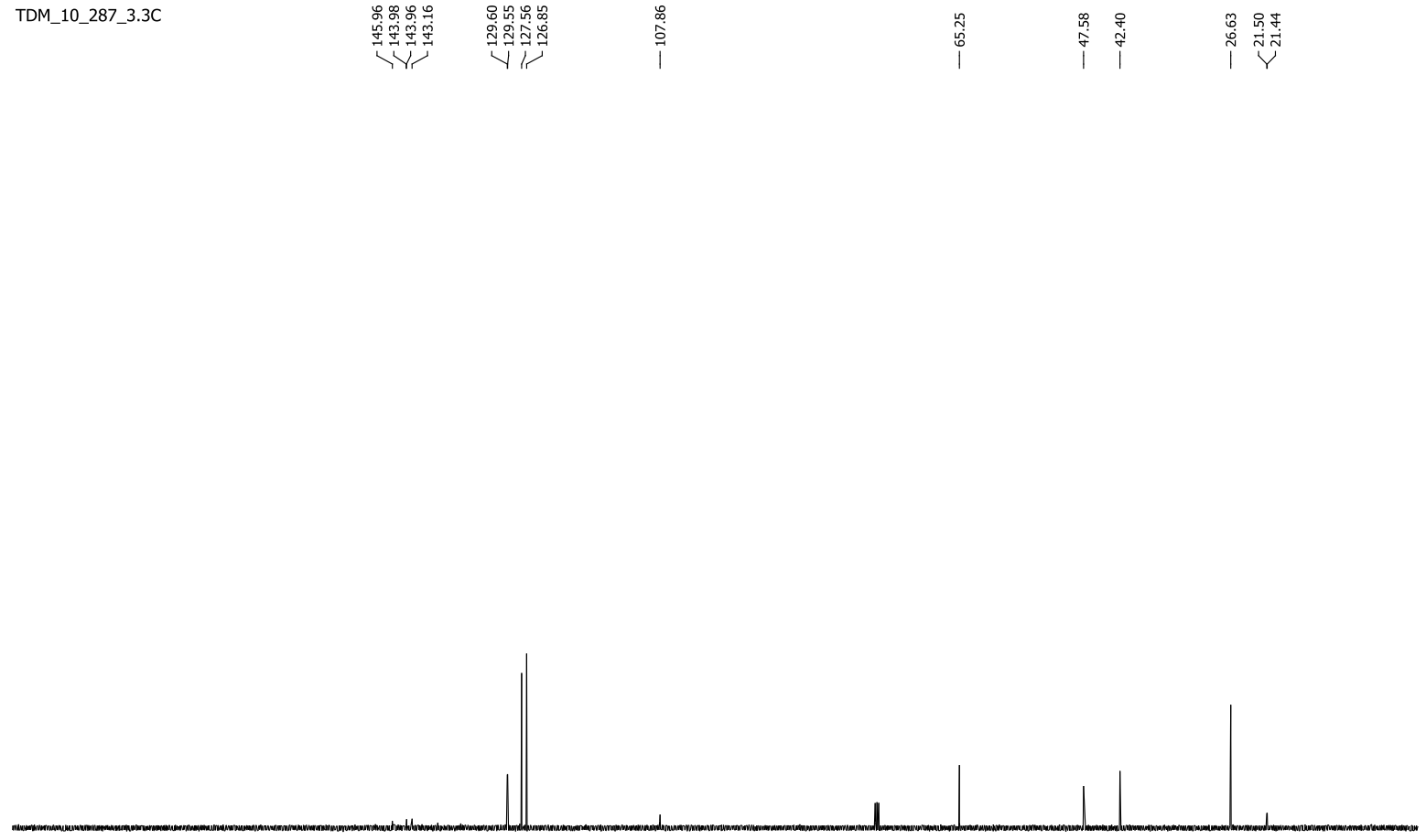

$\begin{array}{lllllll}180 & 170 & 160 & 150 & 140 & 130 & 120\end{array}$

$\begin{array}{lc}110 & 100 \\ \mathrm{f} 1 & (\mathrm{ppm})\end{array}$

$90 \quad 80$

$70 \quad 60$

$60 \quad 50 \quad 40$

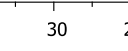

$20 \quad 10 \quad 0$ 

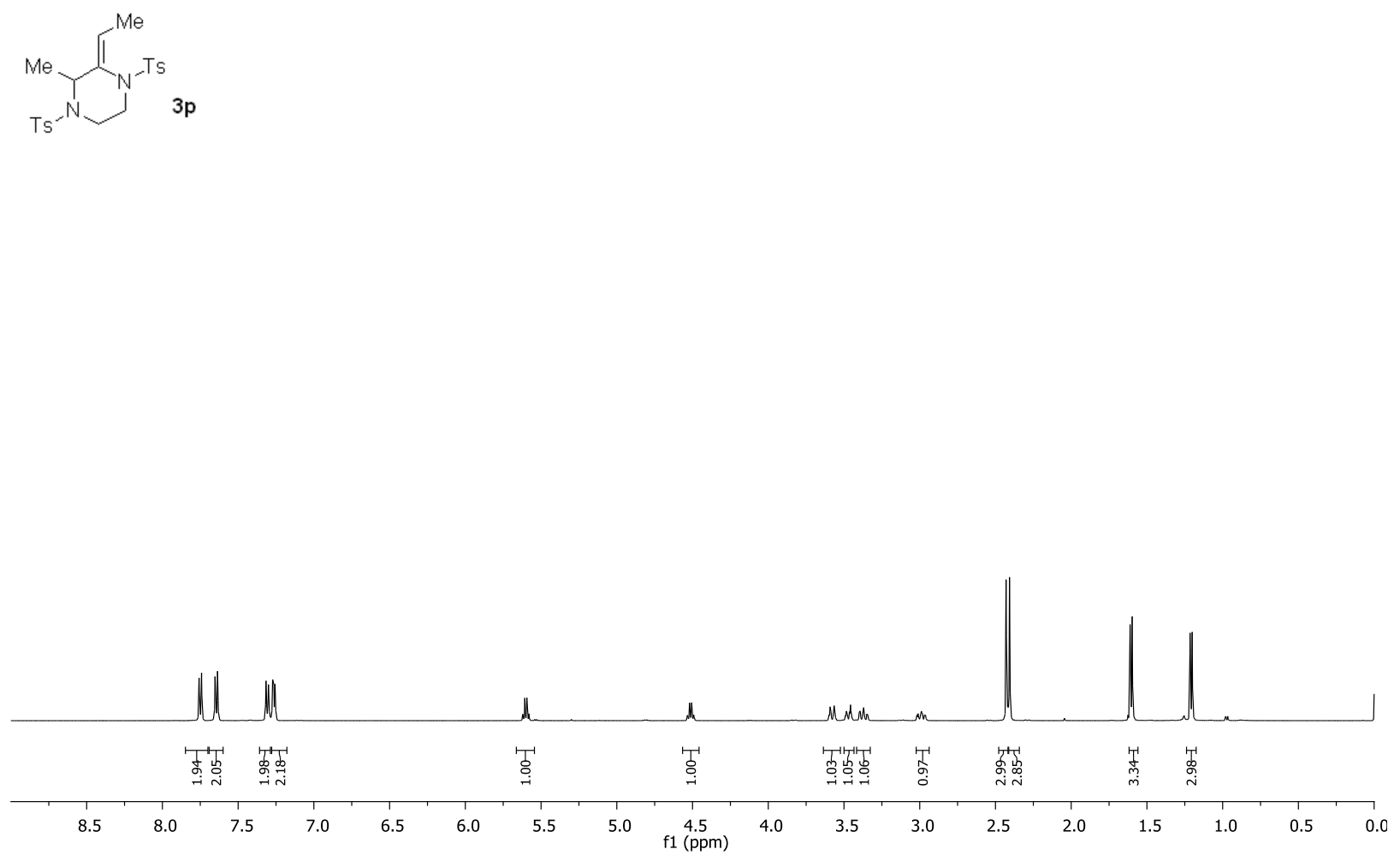

TDM_297_1.2C

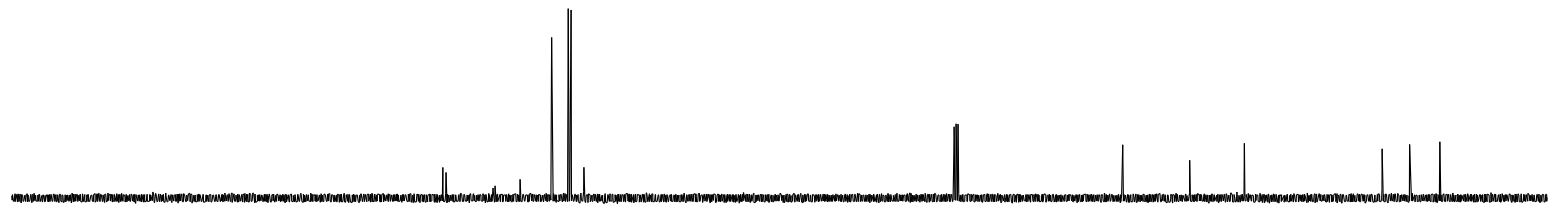

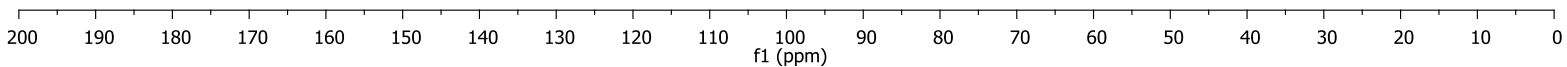




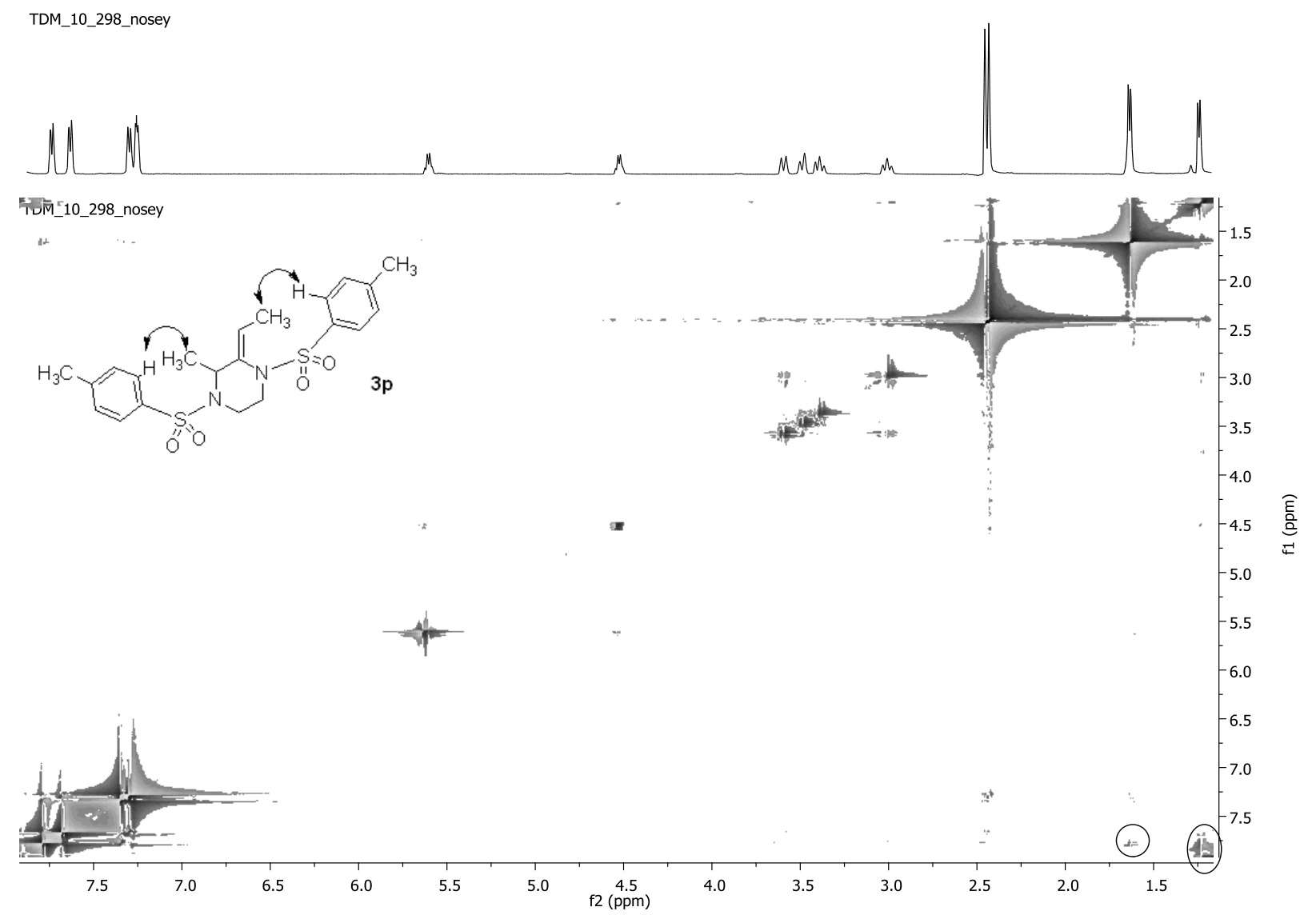


TDM_11_5_2.4

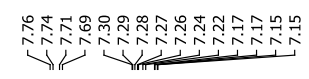

궁ำ

لْن

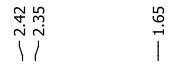

$3 q$

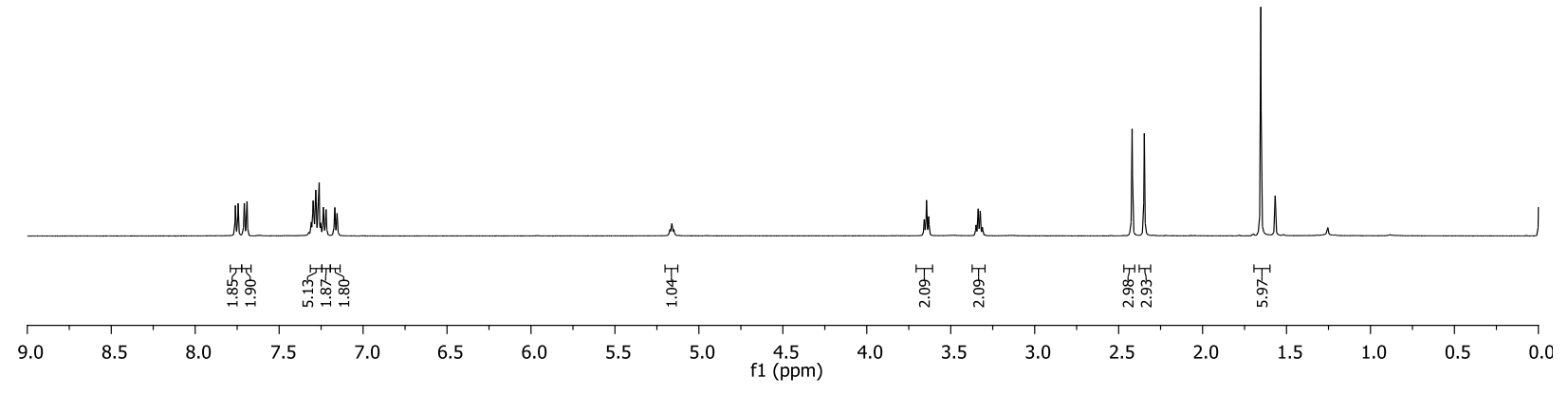

TDM_11_5_2.4C

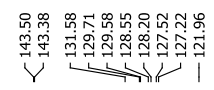

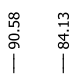

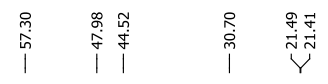

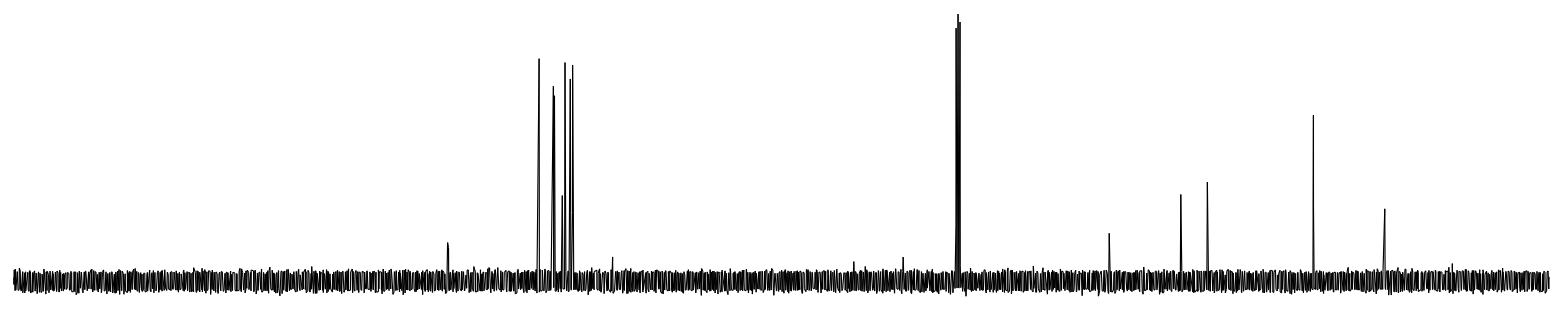

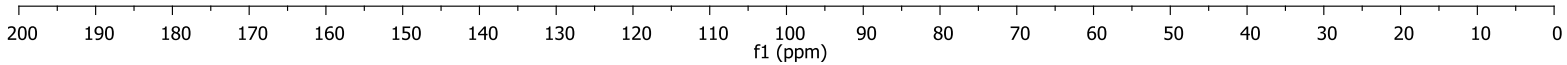




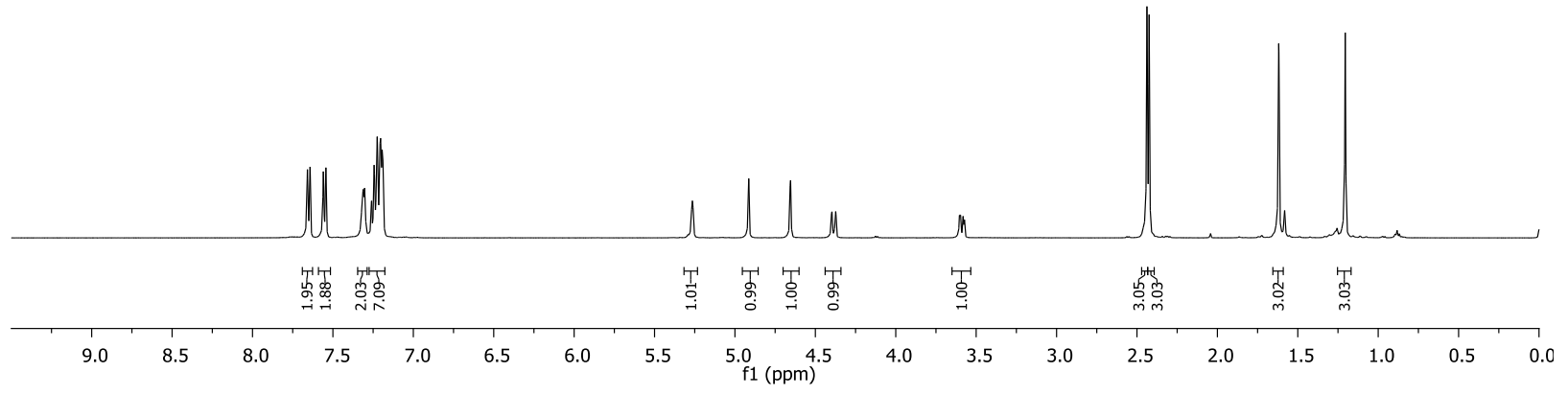

TDM_12_12_1C 

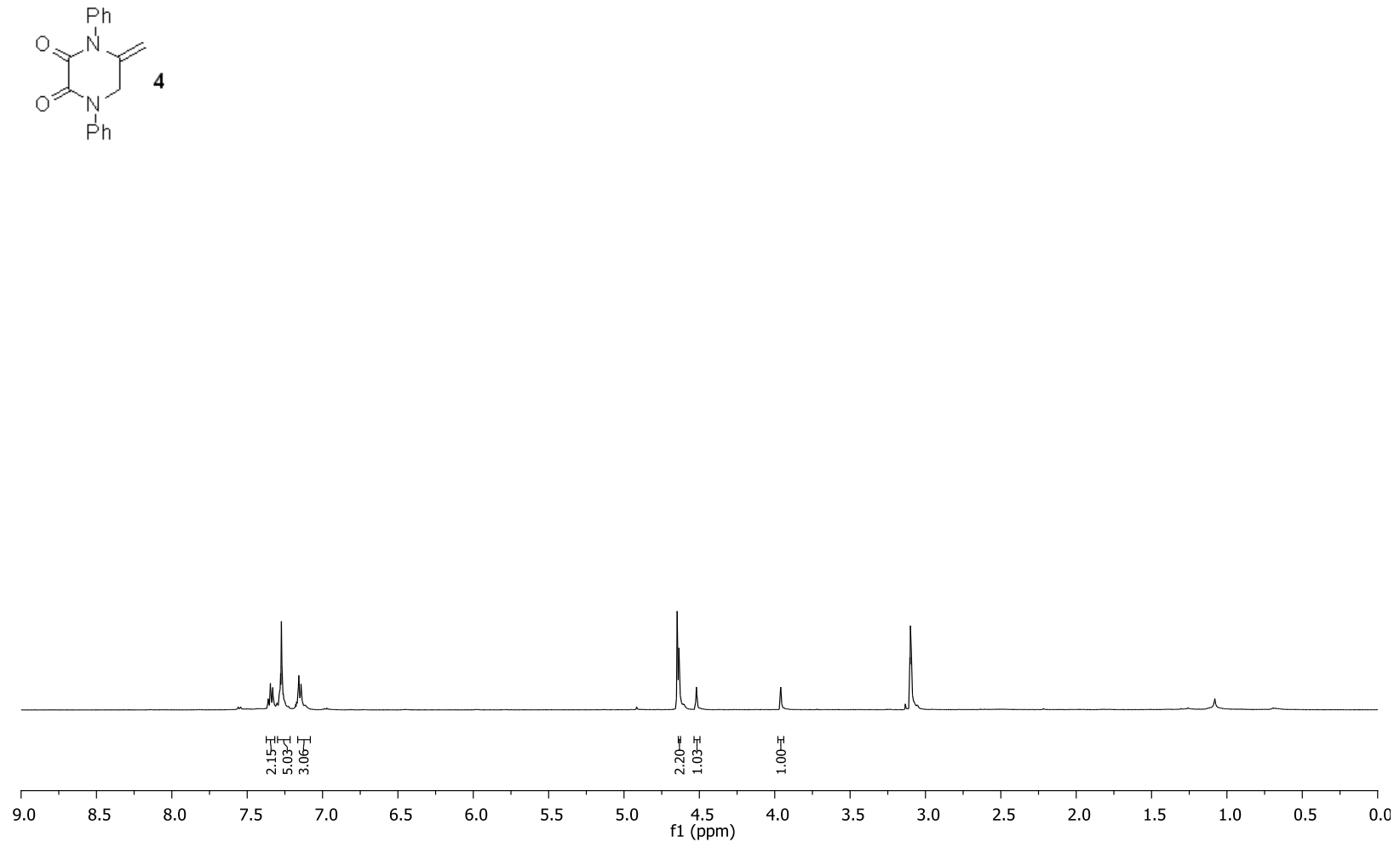

TDM_7_305_1.4C

| ll

sumw w.

$\begin{array}{llllllllllllllllllllllllllllllllllllll}220 & 210 & 200 & 190 & 180 & 170 & 160 & 150 & 140 & 130 & 120 & 110 & 100 & 90 & 80 & 70 & 60 & 50 & 40 & 30 & 20 & 10 & 0 & -10 & -20\end{array}$


TDM_10_291_1.1

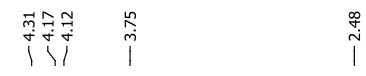<smiles>C=C1CN([13CH3])C(=O)CN1c1ccc(Cl)cc1</smiles>

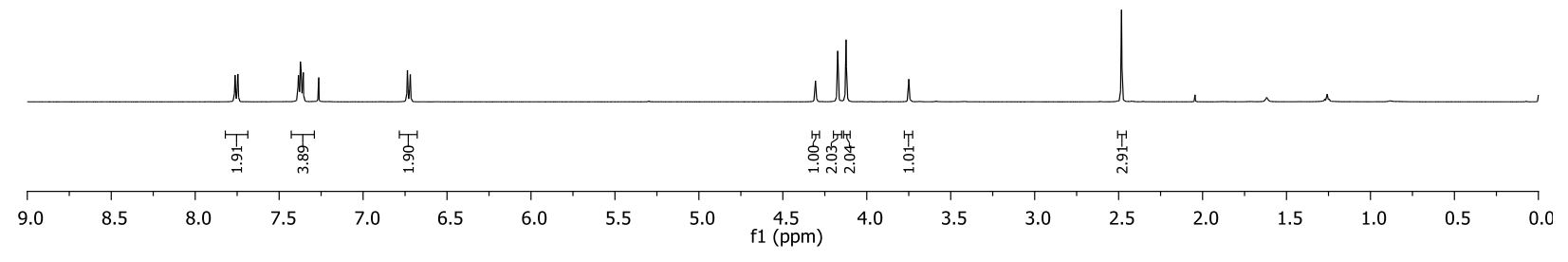

TDM_10_291_1.1C 美

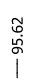

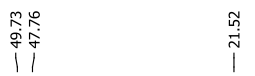

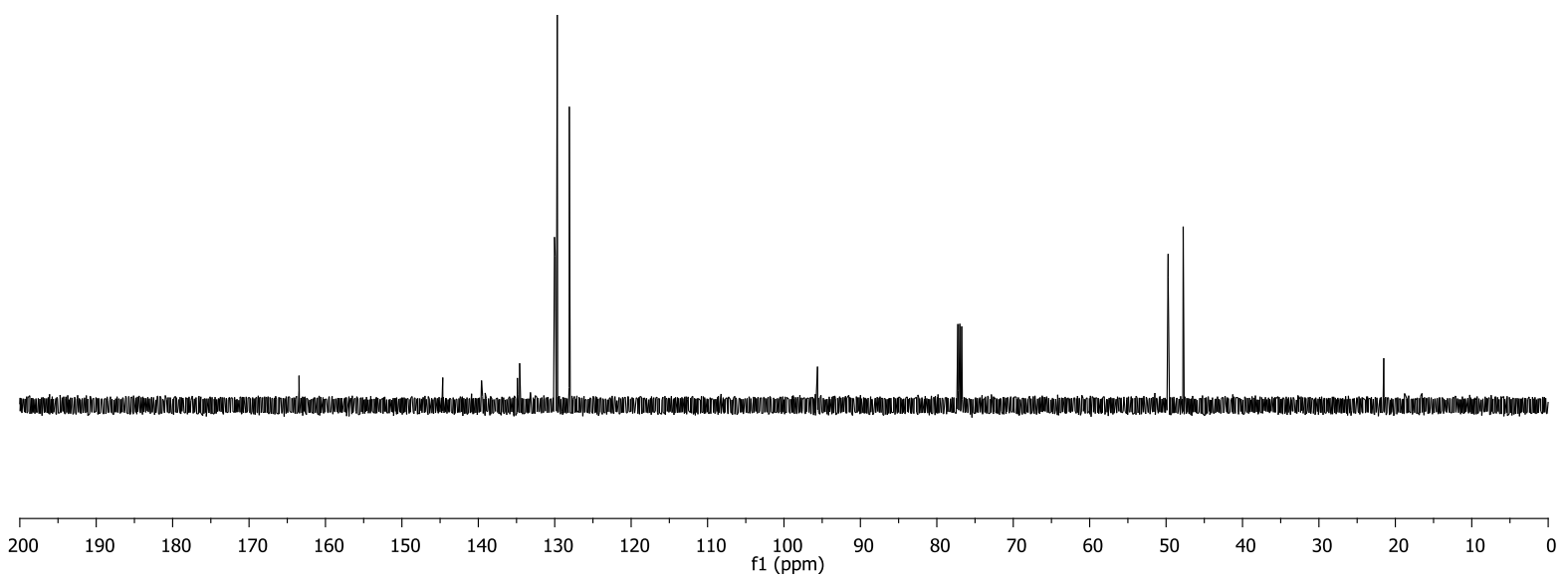


TDM_8_37_1
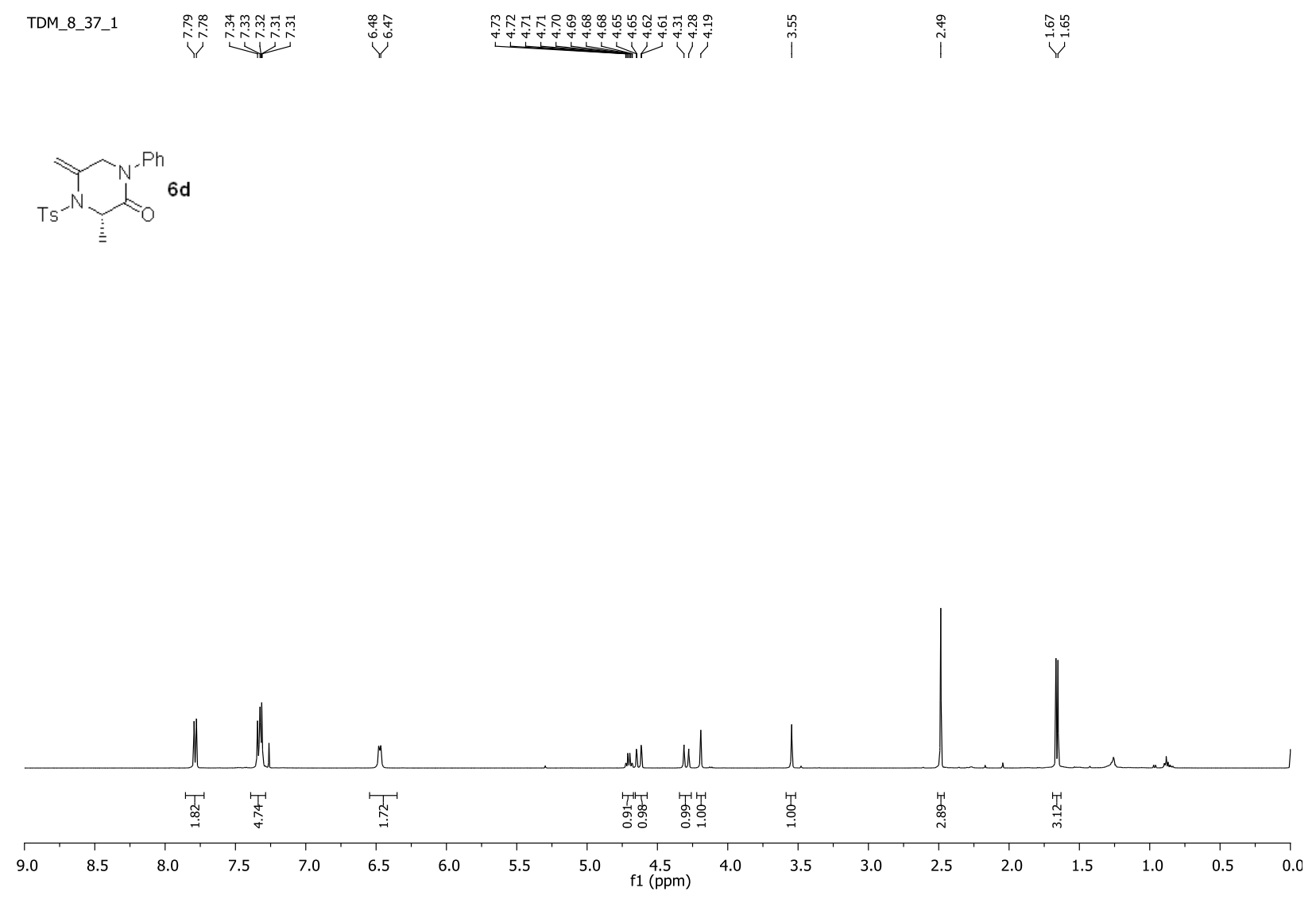

TDM_8_37_1C

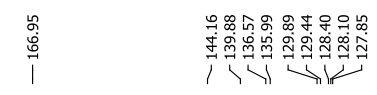

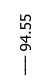

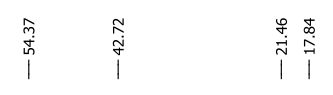
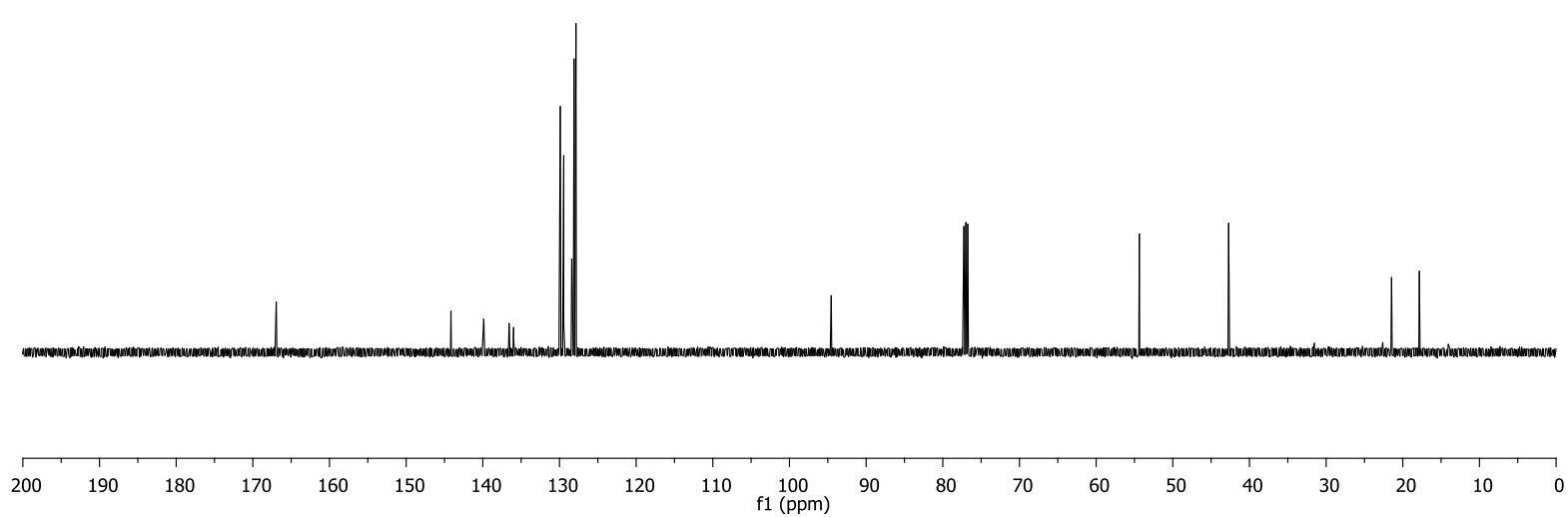

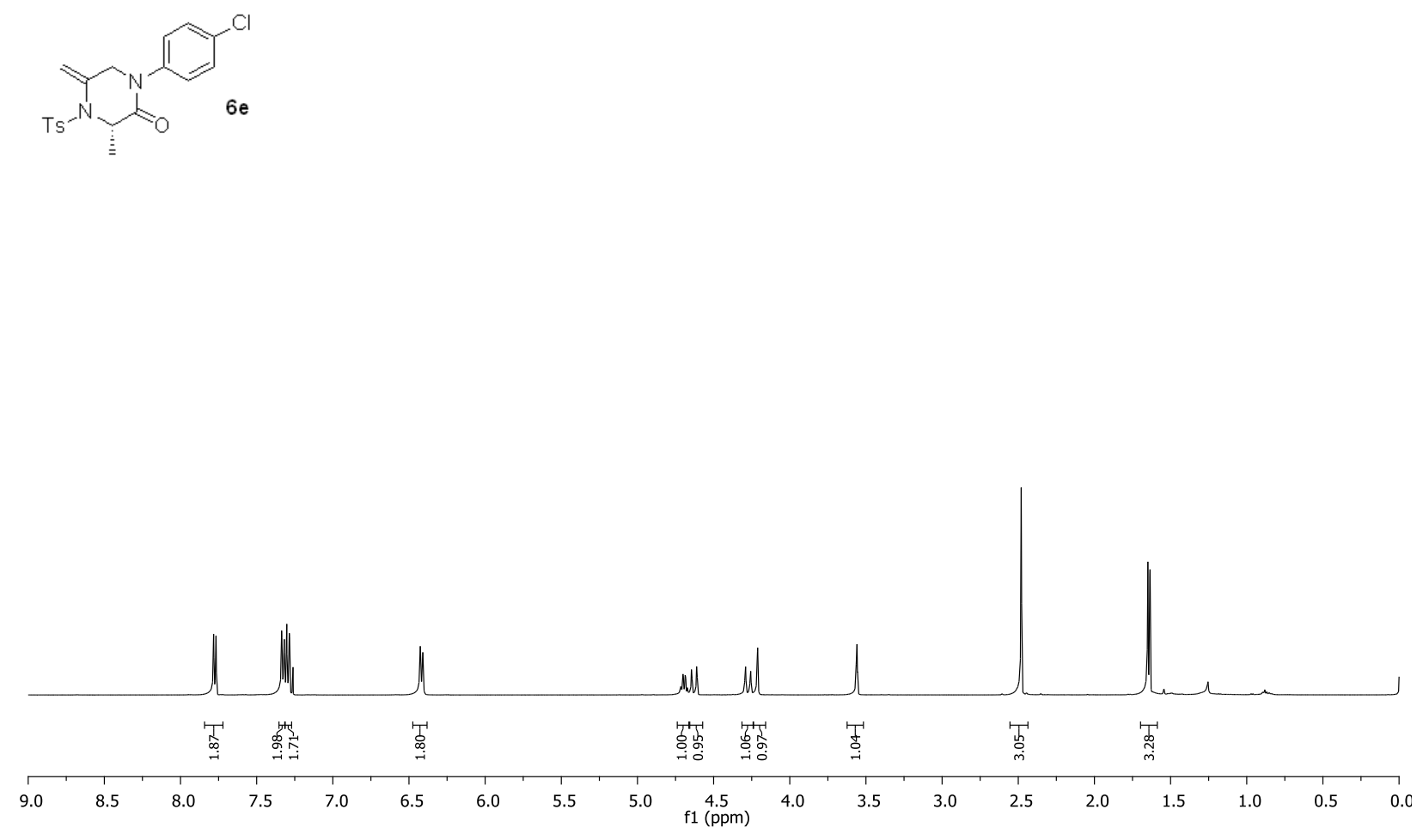

TDM_8_39_2C

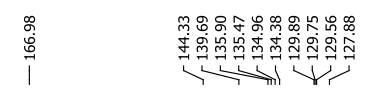

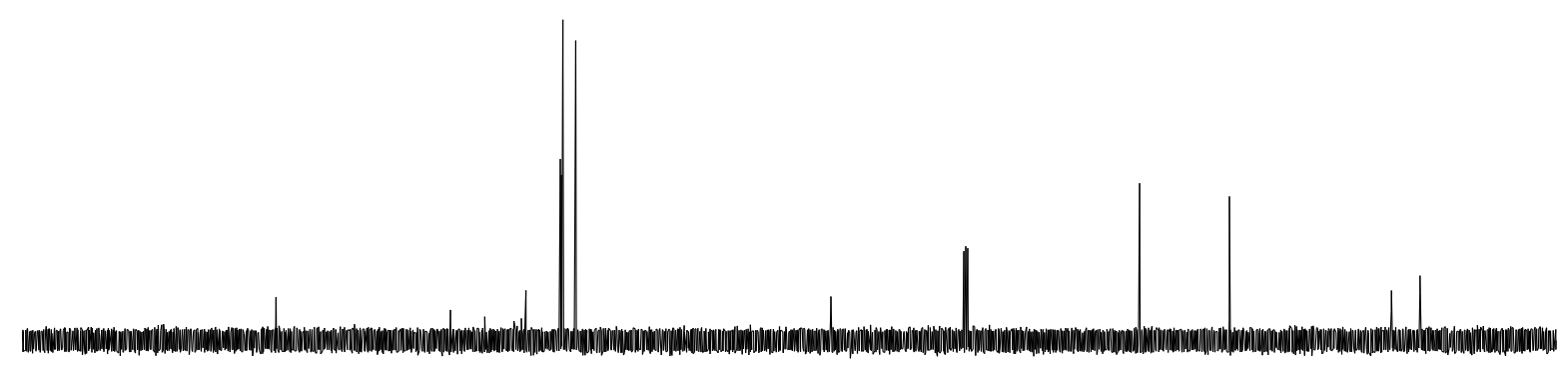

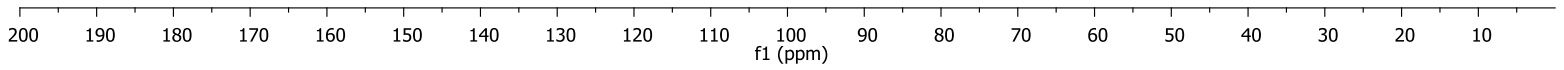




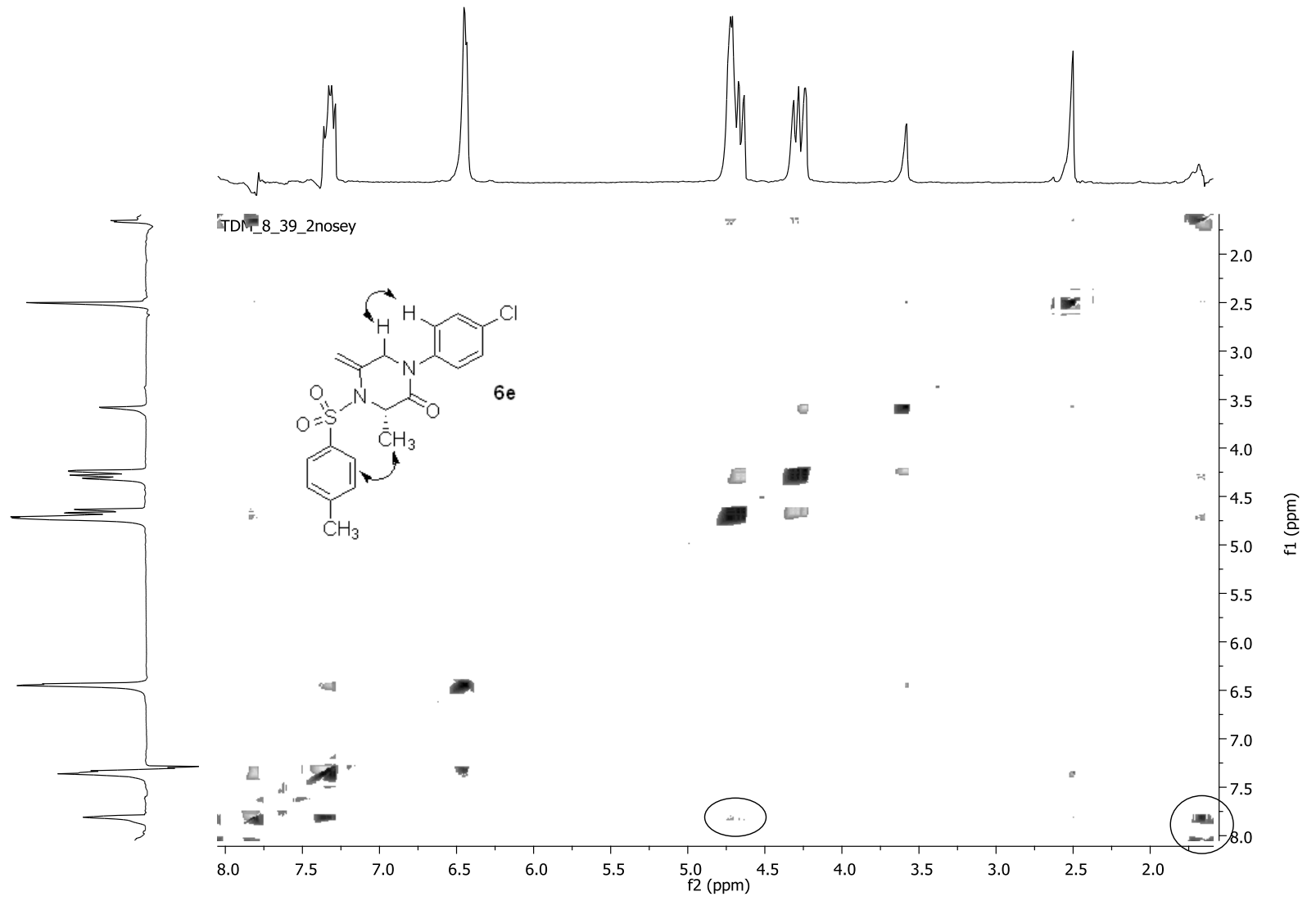



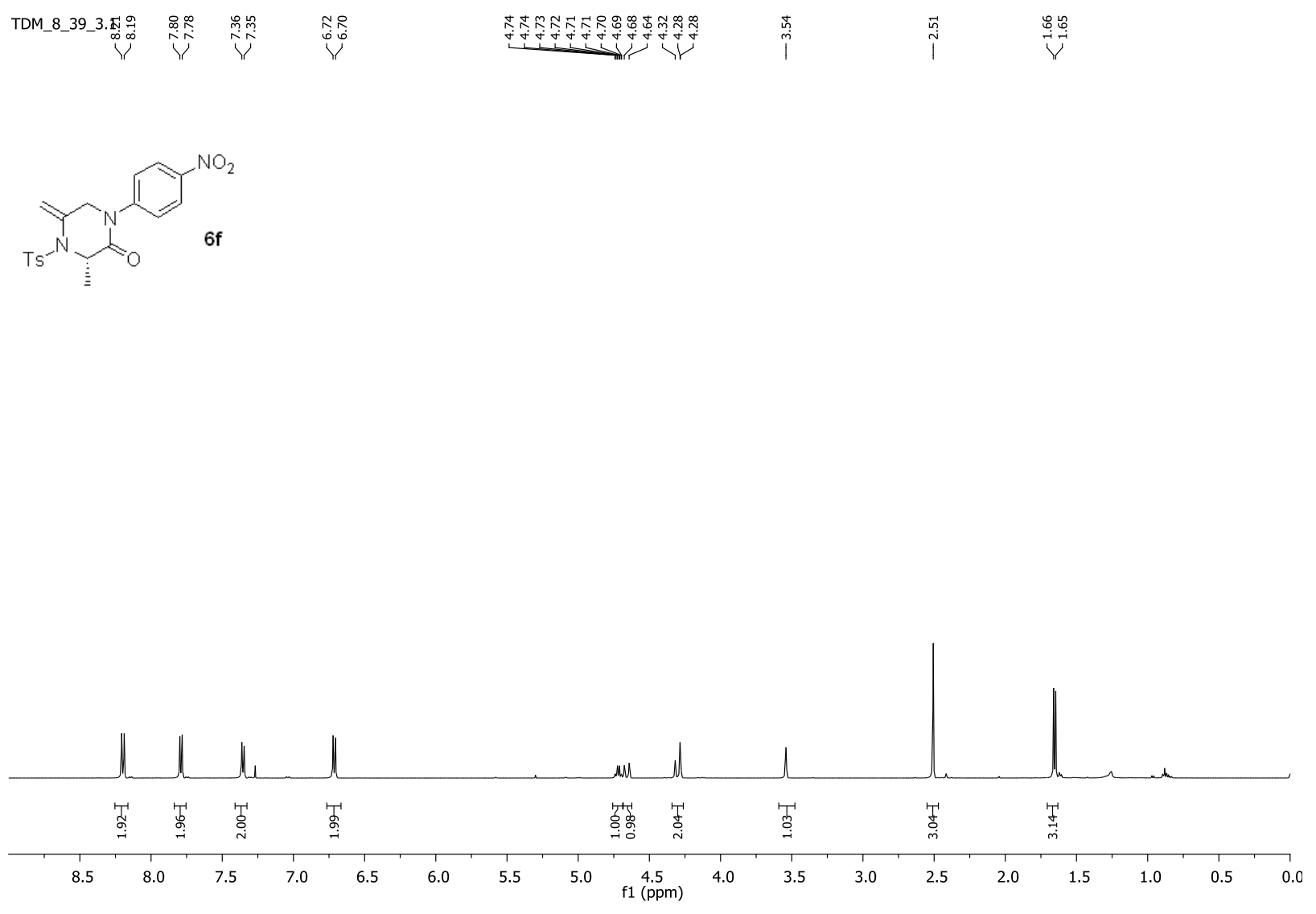

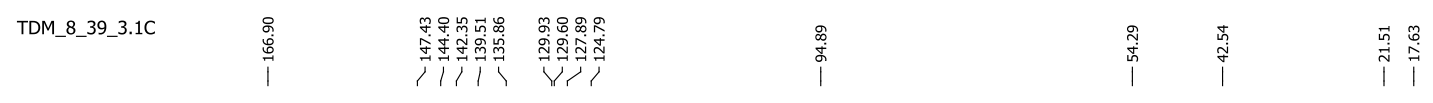
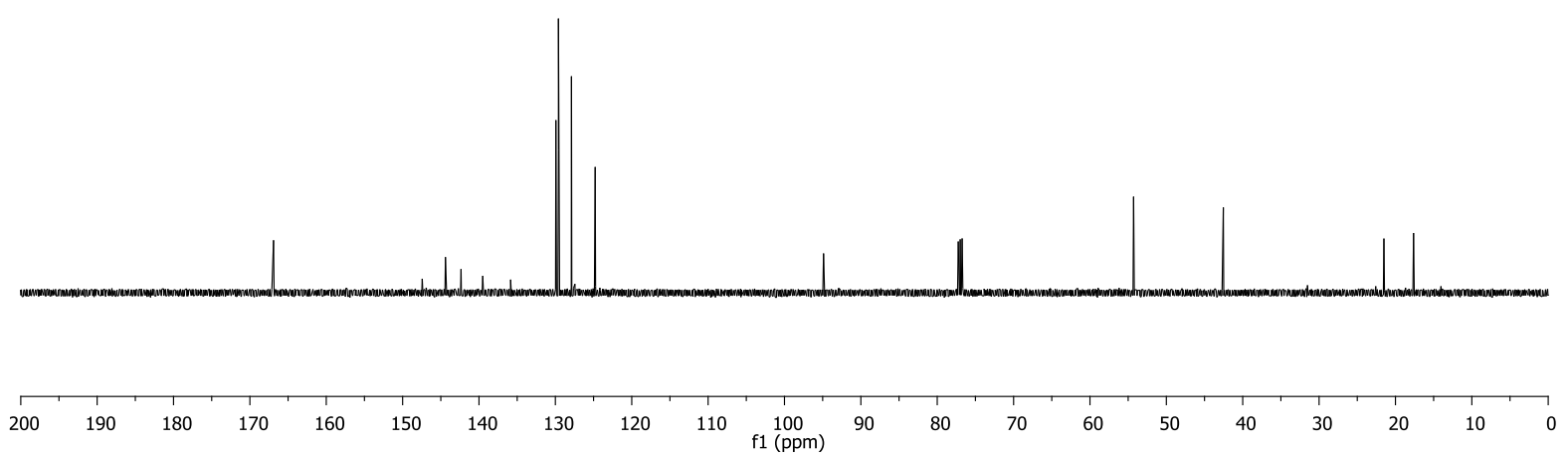
TDM_8_57_1<smiles>C=C1CN(c2cc(C(F)(F)F)cc(C(F)(F)F)c2)C(=O)[C@H](C)N1[13CH3]</smiles>
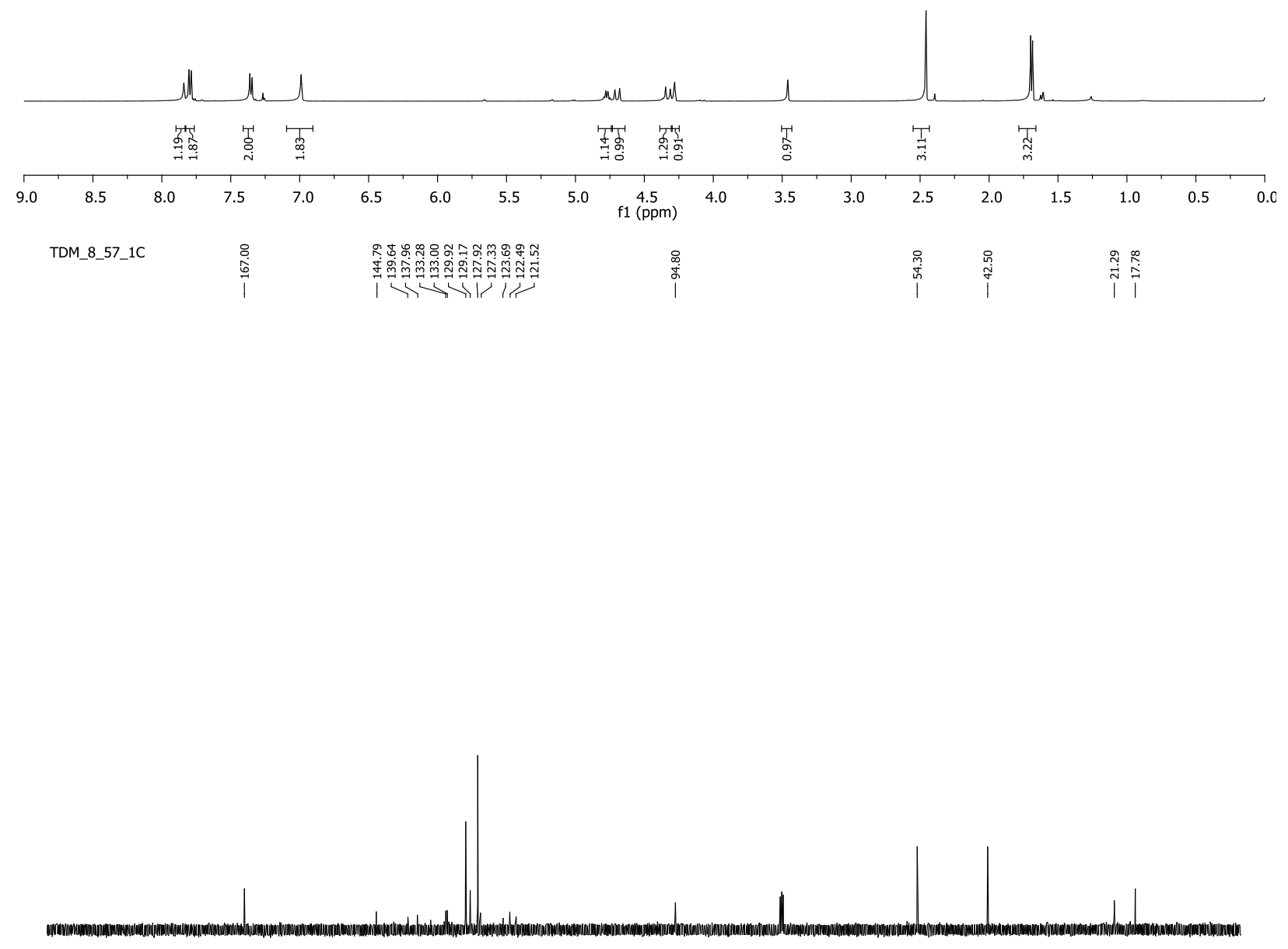

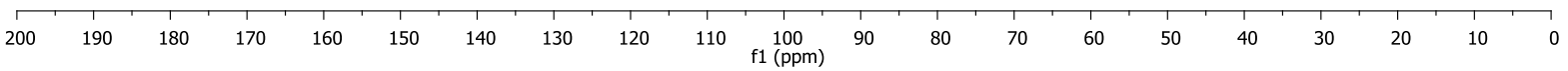



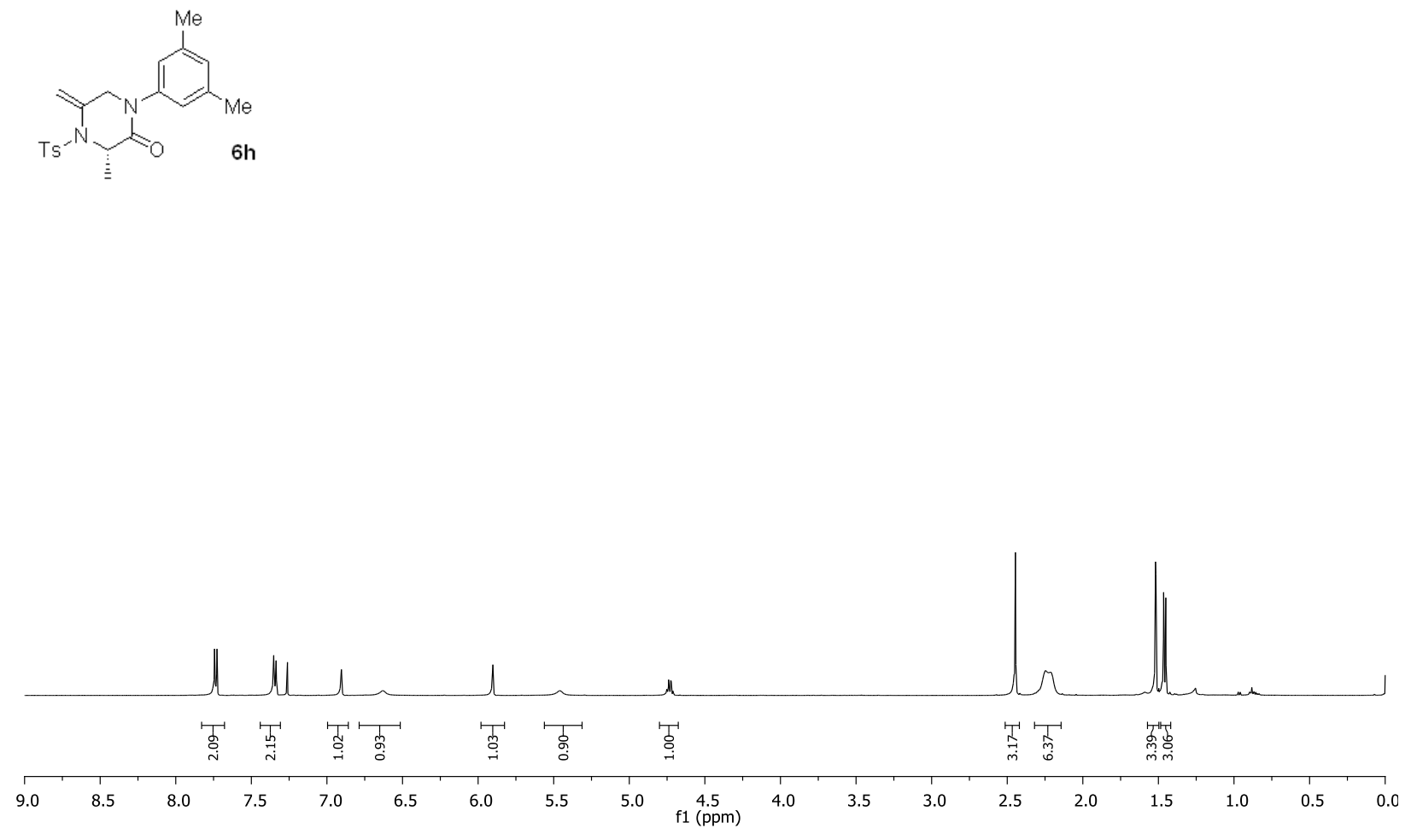

TDM_8_63_2C

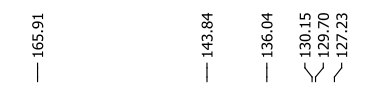

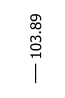

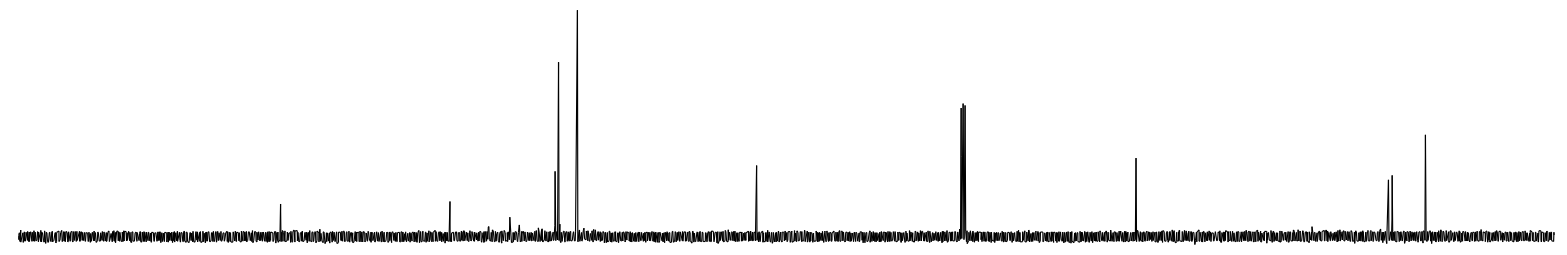

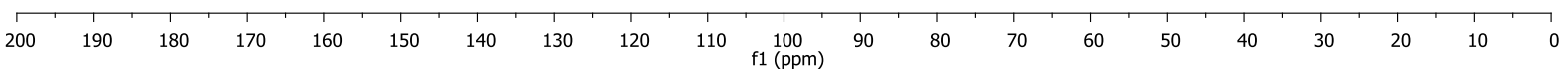




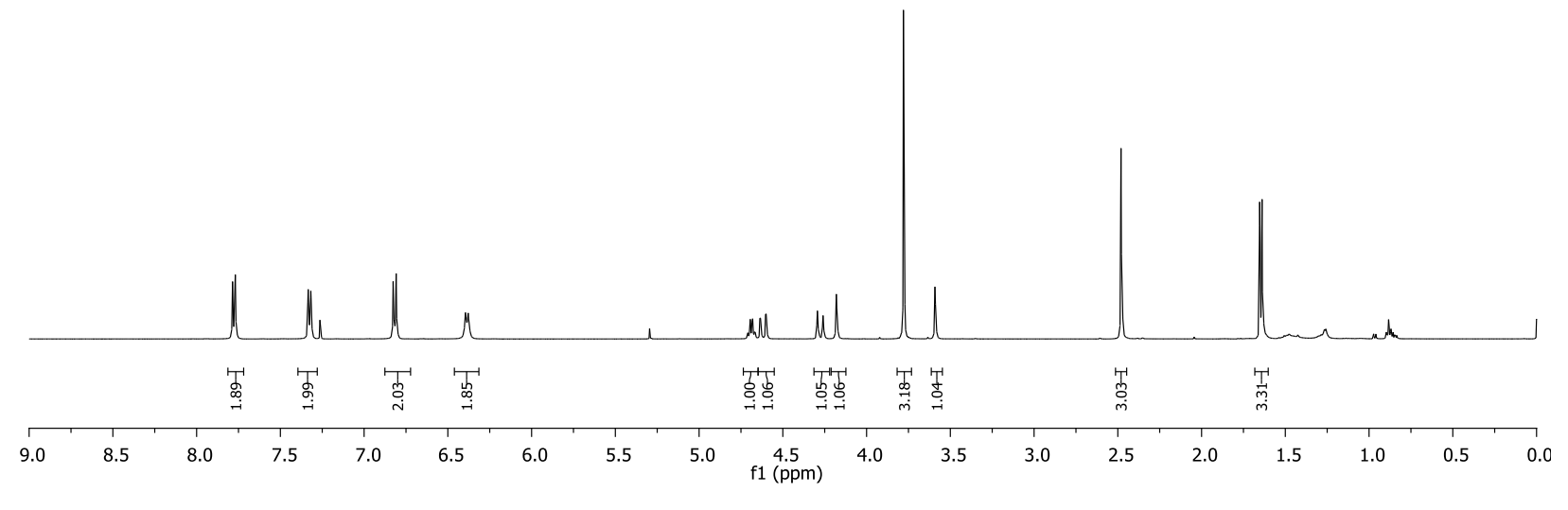

TDM_8_39_11C

|
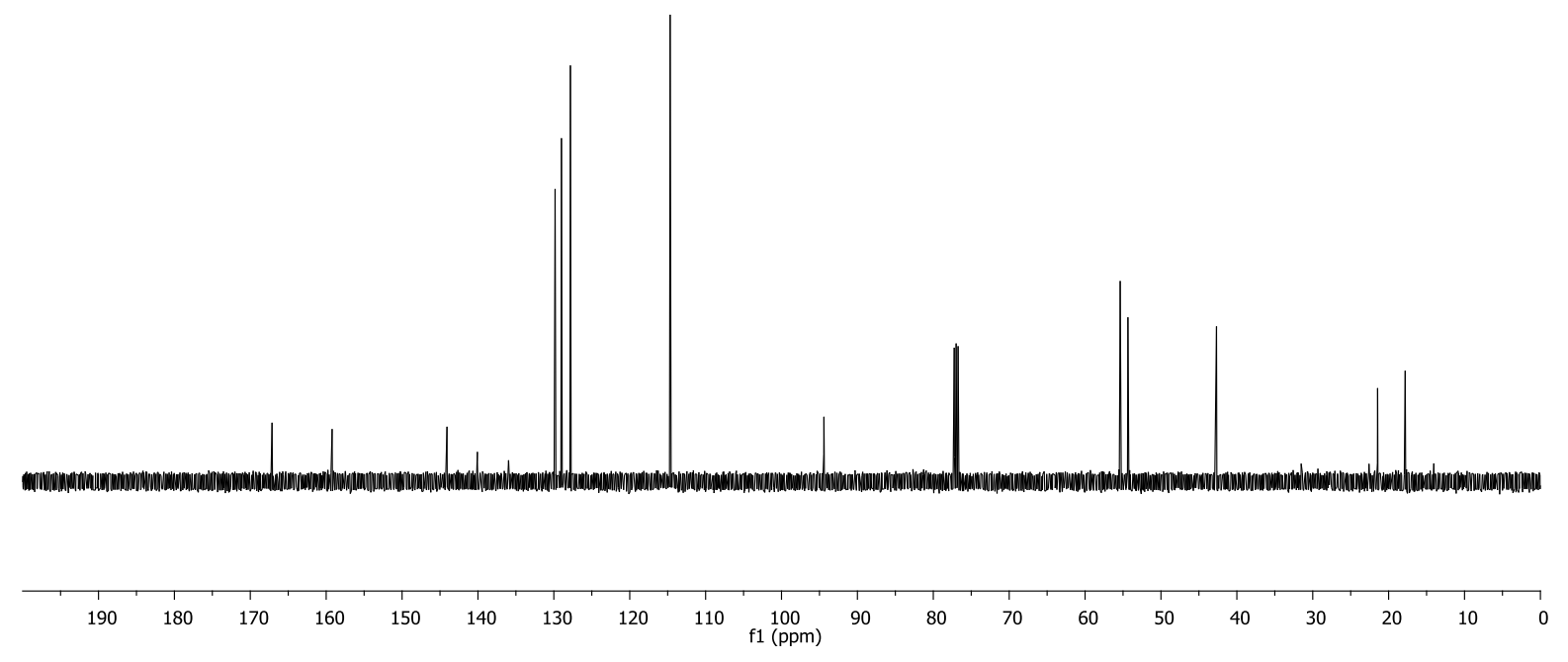


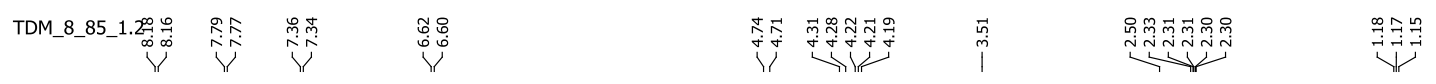<smiles>C=C1CN(c2ccc([N+](=O)[O-])cc2)C(=O)[C@@H](C(C)C)N1[13CH3]</smiles>

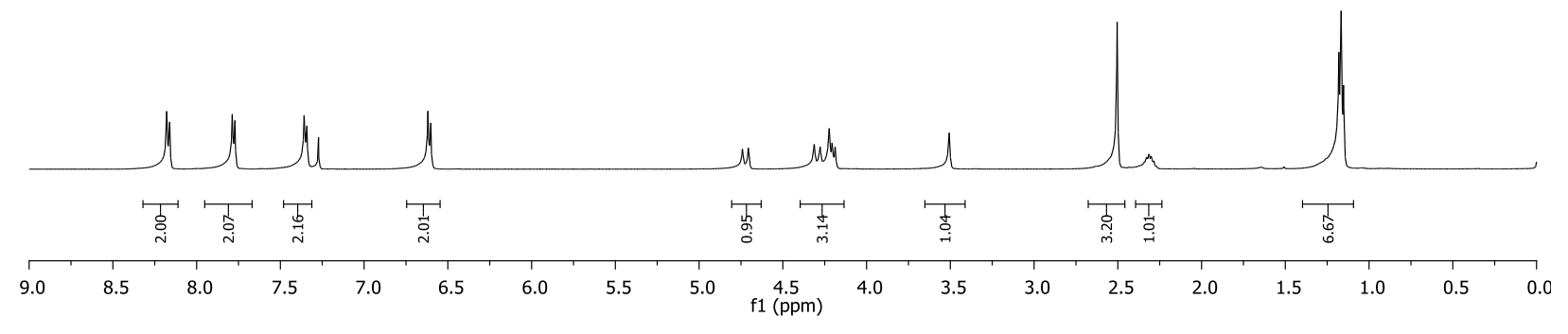

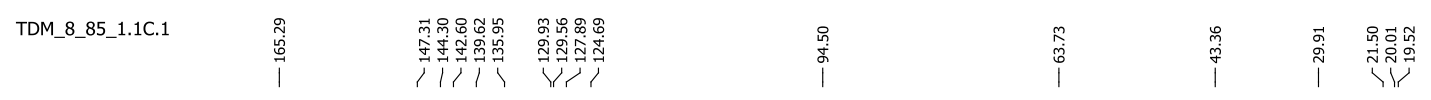

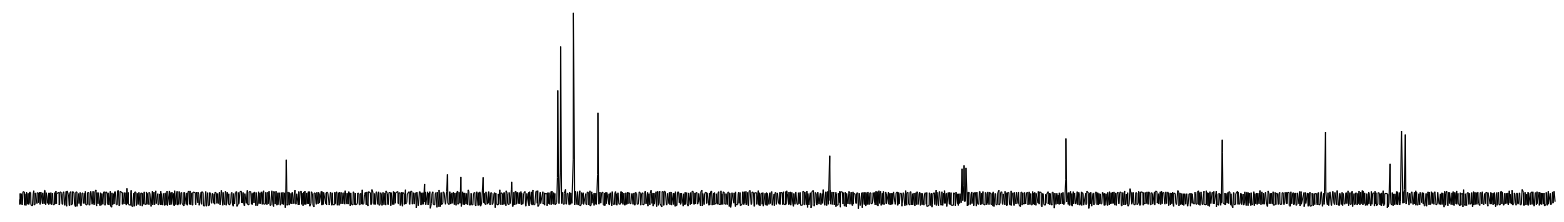

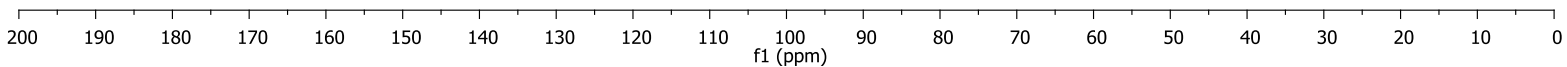


$6 \mathbf{k}$

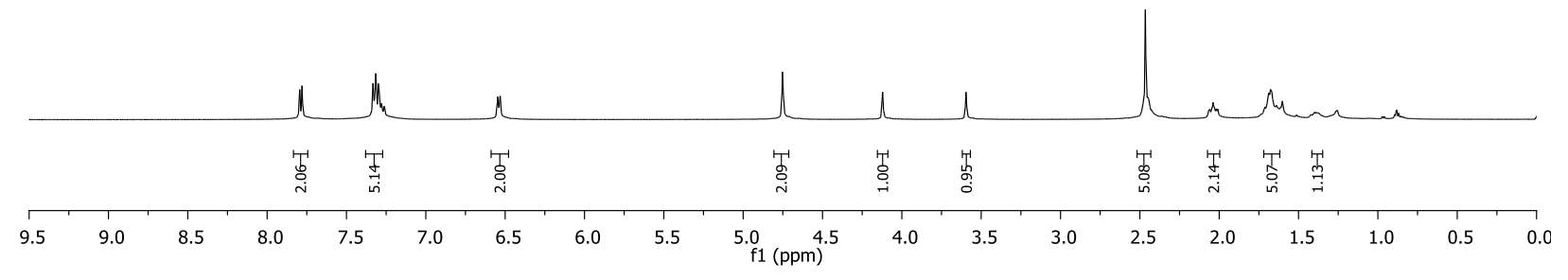

TDM_11_127_1.1C

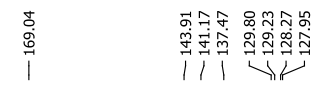

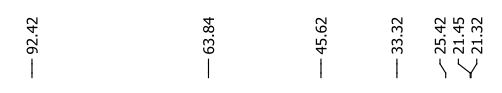

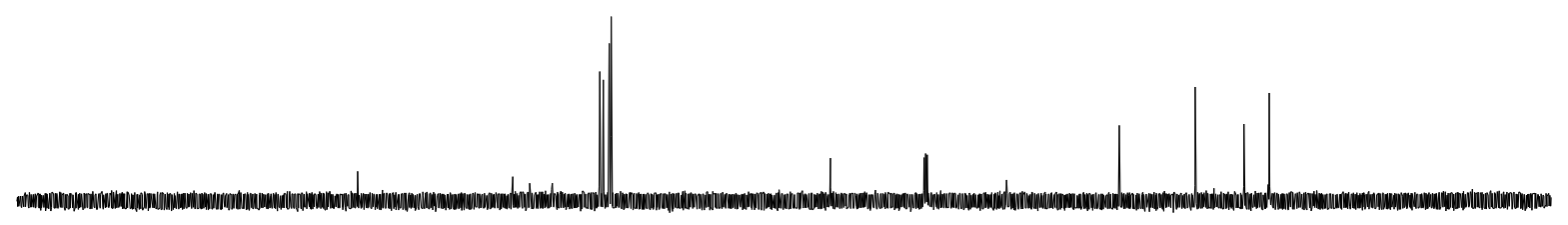

$\begin{array}{lllllllllllllllllllllllllllll} & 220 & 210 & 200 & 190 & 180 & 170 & 160 & 150 & 140 & 130 & 120 & 110 & 100 & 90 & 80 & 70 & 60 & 50 & 40 & 30 & 20 & 10 & 0 & -10 & -20\end{array}$ 

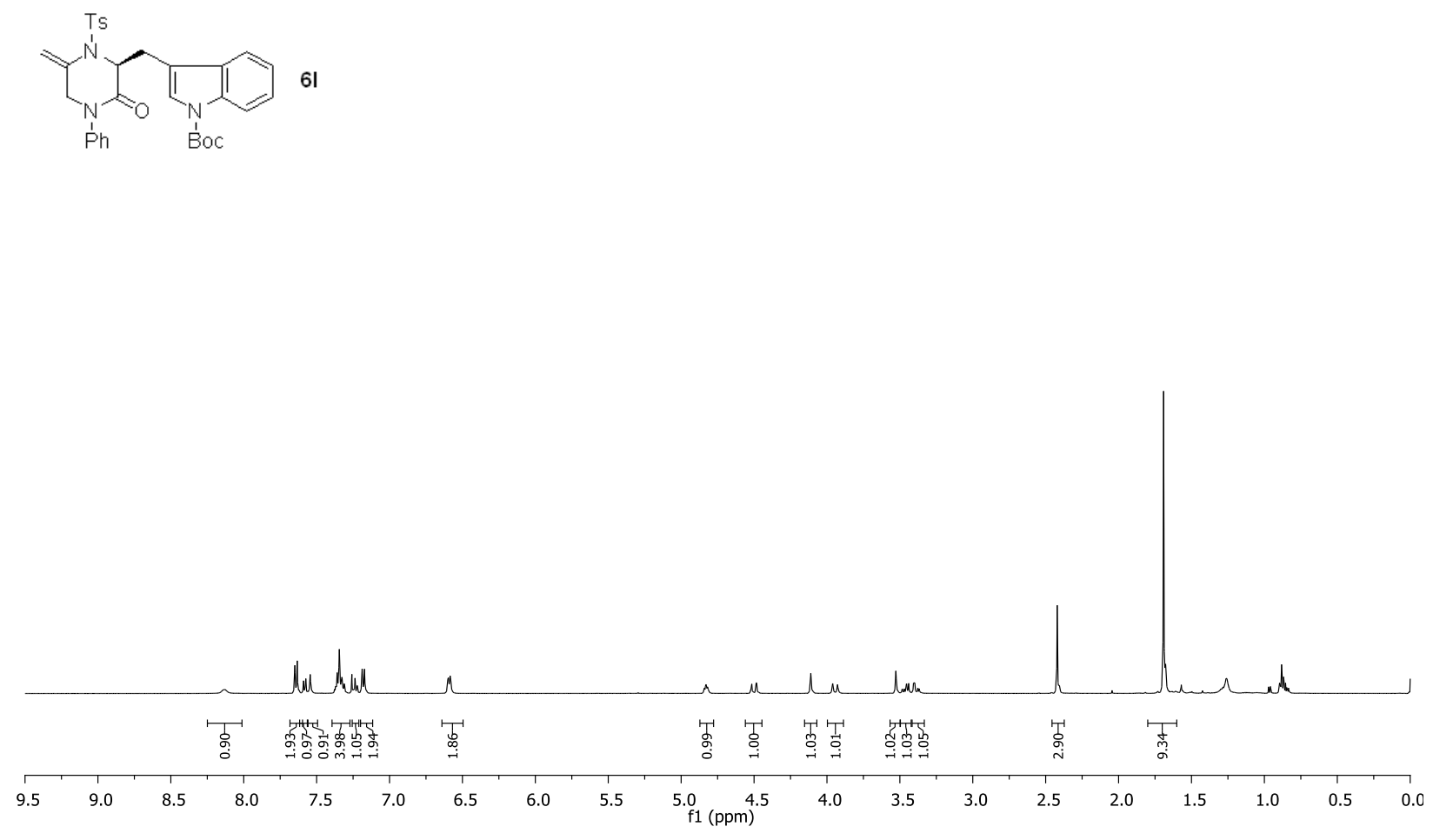

TDM_11_139_1.1C

|

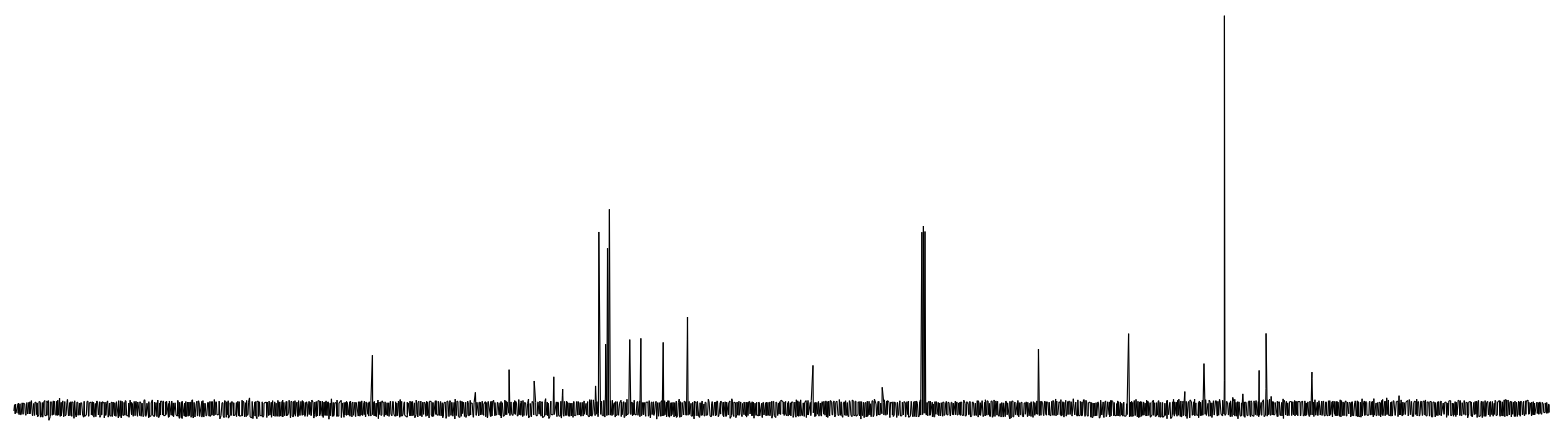

$\begin{array}{lllllllllllllllllllllllllll}220 & 210 & 200 & 190 & 180 & 170 & 160 & 150 & 140 & 130 & 120 & 110 & 100 & 90 & 80 & 70 & 60 & 50 & 40 & 30 & 20 & 10 & 0 & -10 & -20\end{array}$ 
TDM_11_135_1.1

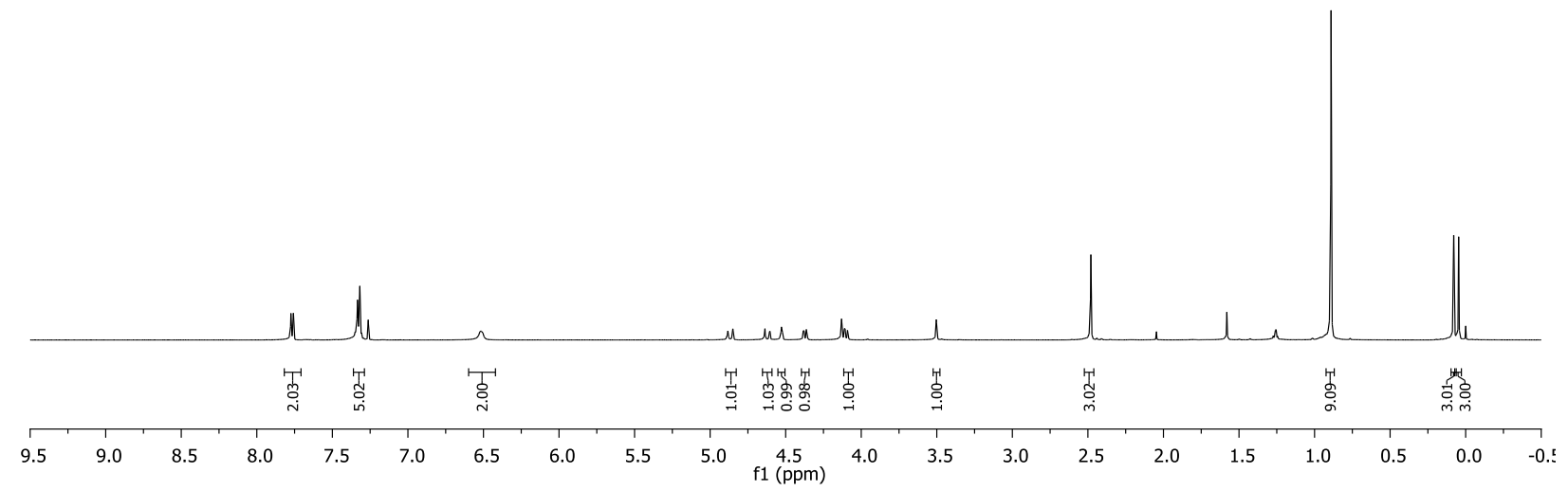
TDM_11_135_1.1C

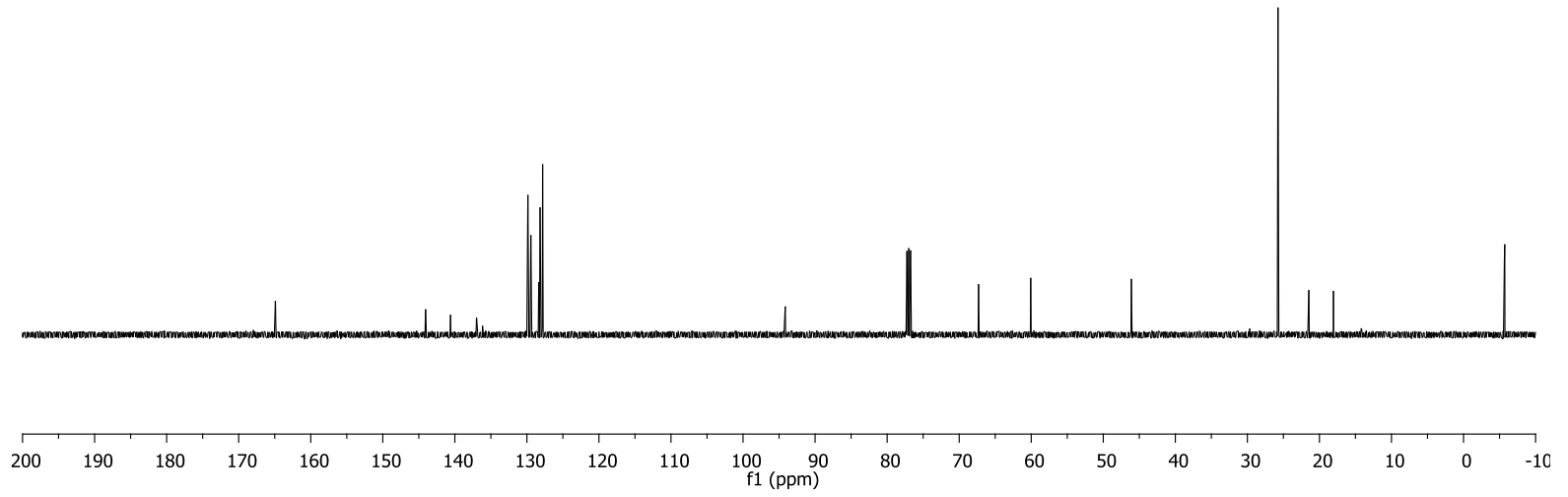



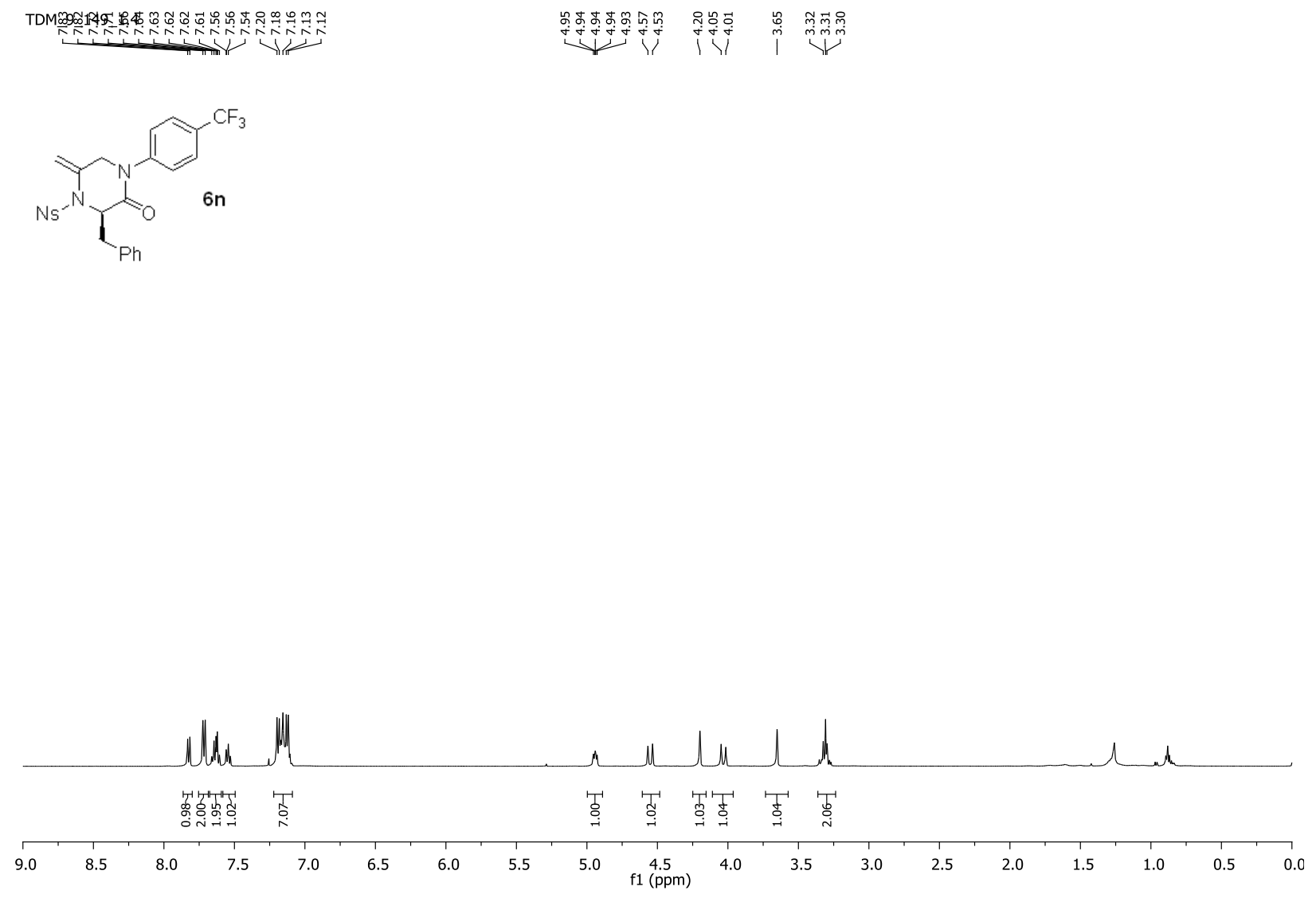

TDM_9_149_1.4C

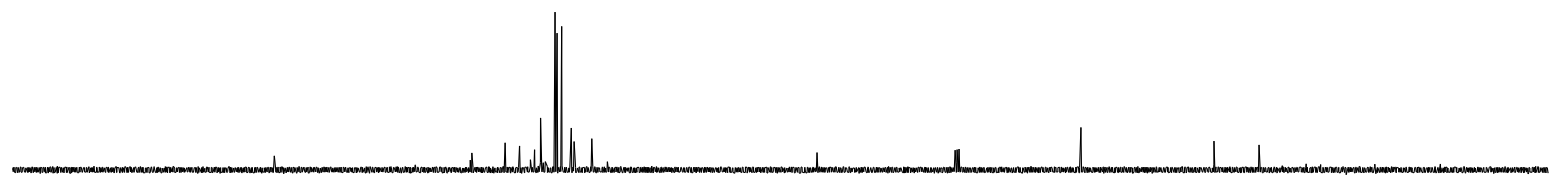

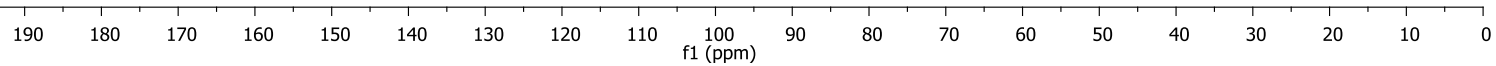



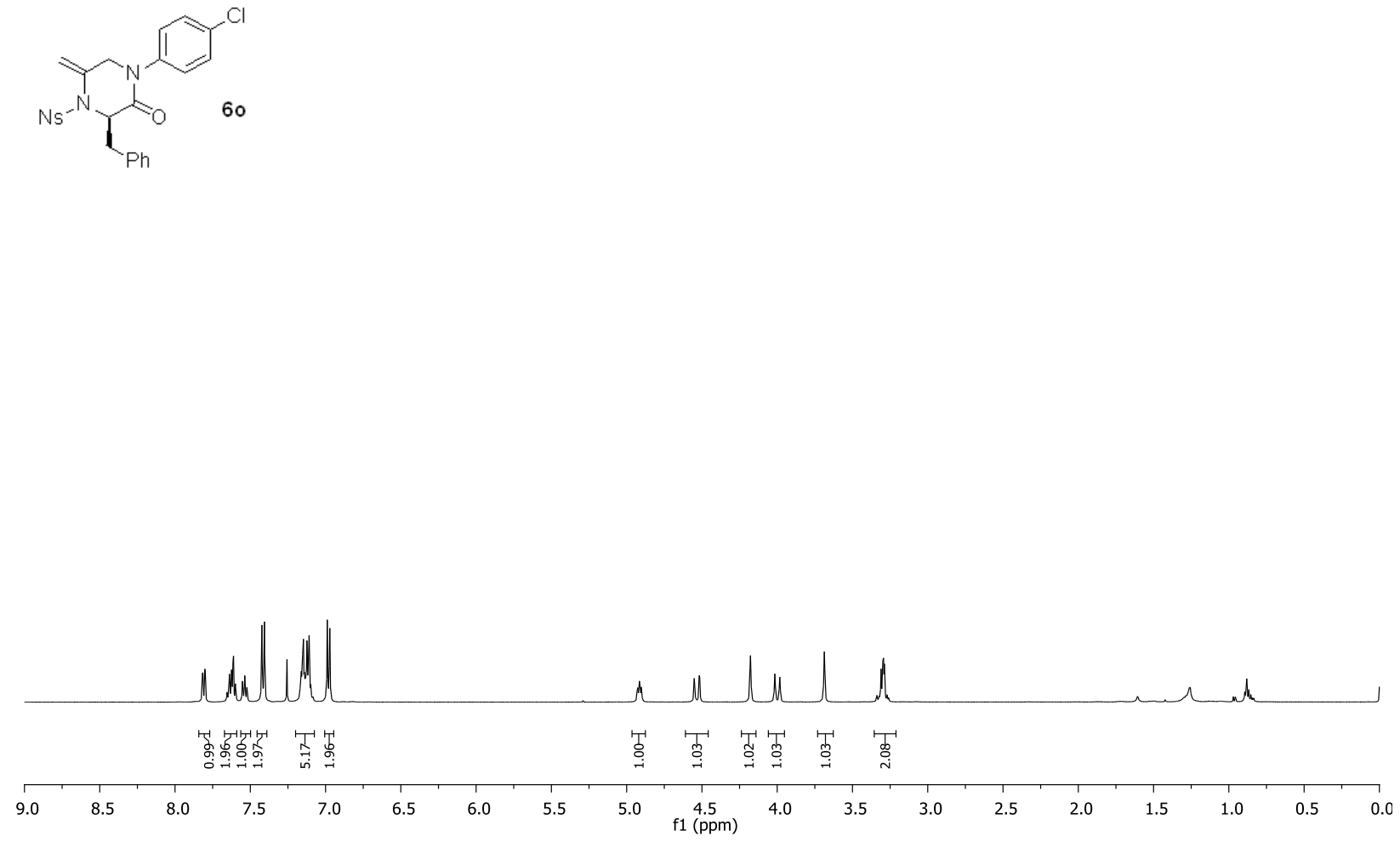

TDM_8_137_1.3C

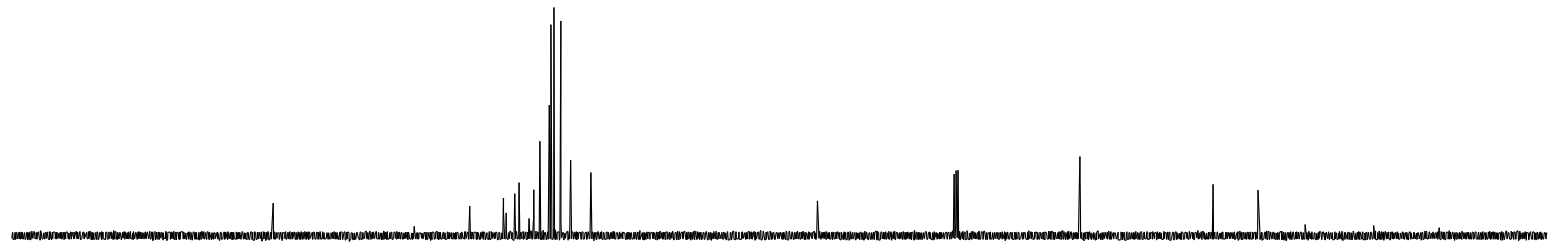

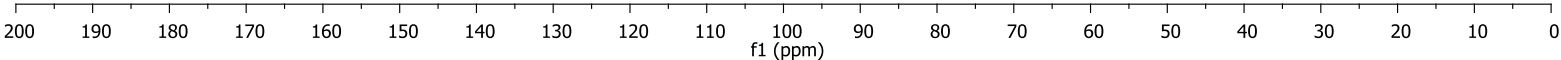



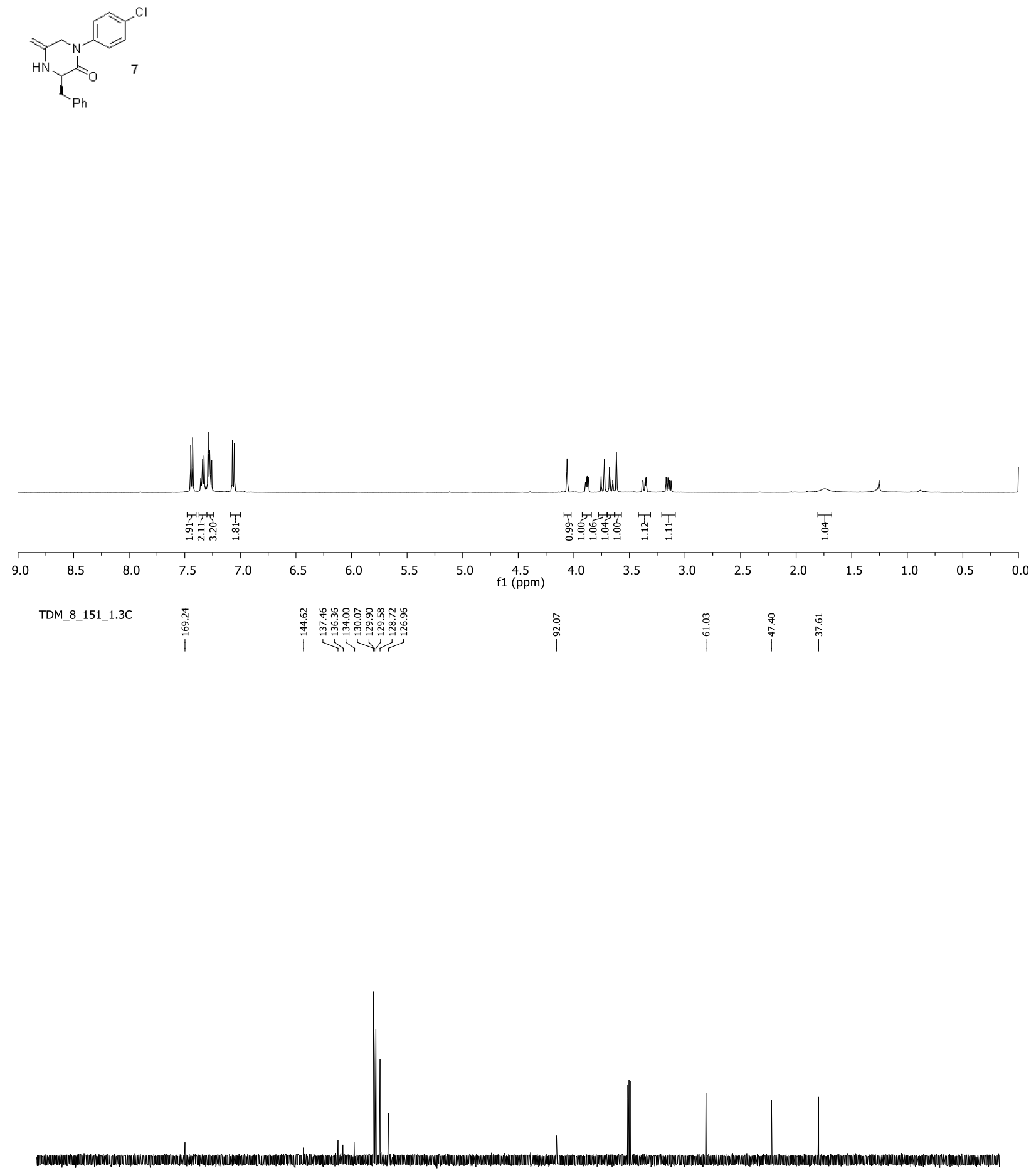

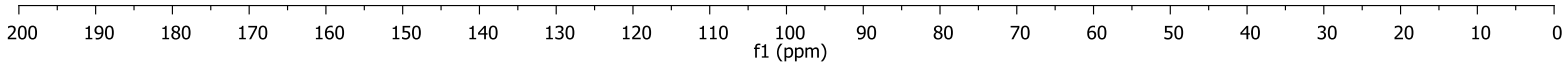

